\title{
INVERSE COMPTON SCATTERING IN GALAXY CLUSTERS
}

\author{
Daniel Ryan Wik \\ Springboro, Ohio
}

B.S., Ohio University, 2003

M.S., University of Virginia, 2006

A Dissertation Presented to the Graduate

Faculty of the University of Virginia

in Candidacy for the Degree of

Doctor of Philosophy

\section{Department of Astronomy \\ University of Virginia}

December, 2010
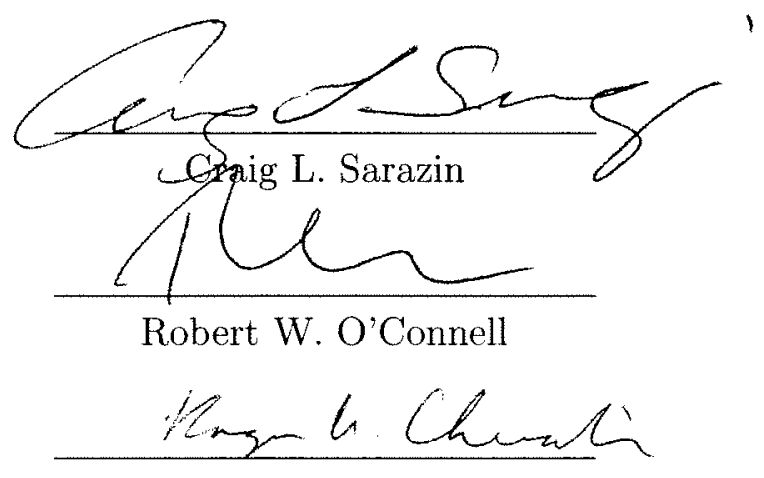

Roger A. Chevalier
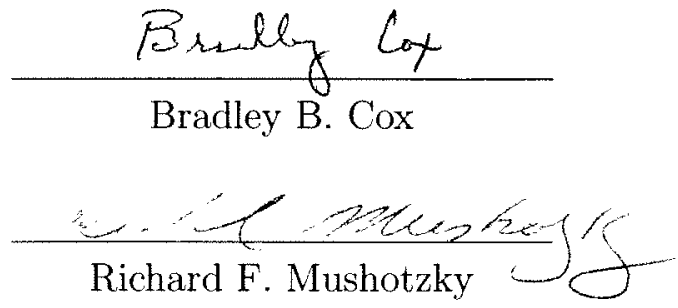


\begin{abstract}
Clusters of galaxies are the largest relaxed structures in the universe and are very important cosmological probes. The state of the intracluster medium (ICM) of galaxy clusters, under the hydrostatic assumption, can be used to infer their masses, the distribution of which can be used to constrain cosmological parameters. Thus, it is important to assess the accuracy of ICM-derived masses. Two phenomena that can bias mass estimates are major cluster mergers, which temporarily removes the ICM from hydrostatic balance, and the existence of energetically significant nonthermal phases of the ICM. In the case of major mergers, we investigate their impact on the measurements of the Sunyaev-Zel'dovich effect which will be used to produce mass estimates. The central (maximum) Comptonization parameter is severely boosted at a level which is similar to the X-ray temperature and luminosity, which leads to several tens of percent bias in cosmological parameters. On the other hand, the integrated Comptonization parameter is found to be robust to mergers, allowing the dark energy equation of state to be accurately determined. Regarding the nonthermal phase of the ICM, we aim to test previous detections of inverse Compton (IC) emission and to make new ones. Taken in conjunction with detected radio synchrotron emission, the relativistic energy content of the ICM can be assessed. We search for IC emission, in the Coma cluster with a detailed analysis of data from two hard X-ray sensitive satellites, Suzaku and Swift, and complimentary data from XMM-Newton. We do not detect IC emission, and our upper limits exclude the previous detections at the $\gtrsim 90 \%$ level. The Swift BAT observations also exclude the possibility that Coma has very strong but very extended IC emission. Similarly, no significant IC emission is detected in spectra from the Swift BAT survey of a sample (HIFLUGCS) of the brightest X-ray clusters, in contrast to expectations based on the results of previous
\end{abstract}


searches. A weak detection of IC emission from clusters with radio halos and relics is found from the Swift survey. The implications of this work, along with future prospects, are also discussed. 


\section{Acknowledgements}

As with any involved, long term endeavor, this work and all the work leading up to it does not solely result from the effort of one individual. It is only made possible with the help of a supportive community, in terms of its history, culture, and most importantly all the people that carry on those traditions. I cannot adequately thank or acknowledge all those deserving mention, nor will I attempt to, but I will offer that it would be a great understatement to say the bootstraps by which I have pulled myself up were well made by those that came before and well maintained by those that came with me on this journey.

First, I must admit that none of the research presented herein would have happened without the ideas, insight, dedication, and all-around awesomeness of my mentor, Craig Sarazin. His distinguished career, which includes literally writing the book on galaxy clusters, has lead the field for decades and been an excellent guide for me for, thankfully, not quite so long. I have to credit Craig for the majority of my professional development, both scientifically and practically, which is due in no small part to his incredible openness, accessibility, and willingness to pause whatever he is doing if you pop into his office to chat. I look forward to working with Craig for years to come.

But neither this work, his work, or any of the discoveries in X-ray astronomy would have been possible without the generous public support necessary to launch our instruments and telescopes into orbit. Specifically, I am indebted to the creators and funders of projects like Chandra, XMM-Newton, Suzaku, and Swift, with which the data presented here was gathered. These missions allow us to understand our place in the universe, in my opinion providing a context for finding meaning in life, both for individuals and the human race as a whole. I am proud to live in a society 
that values such endeavors and understands how valuable and inspiring exploration is. Also, I am proud that this field is full of so many dedicated and brilliant fellow scientists, and it has been my pleasure to collaborate with them; I decline to list them here so you will look up the papers that derive from the following chapters and hopefully discover all the fine work they have done elsewhere as well.

I must thank the rest of my thesis committee for their guidance throughout, and the clearly excellent advice that on my trips to NASA's Goddard Space Flight Center I should interact with as many people as possible - because that would be a great way to land a job - which I have indeed done. Such advice and general support came not just from my committee but from the Astronomy Department as a whole, about which I cannot think of one single negative thing to say. The environment here at UVa, created by a spectacular community of scientists, students, and supporters, is incredibly friendly, nurturing, and creative. I know I should have been dying to escape the long hours, low pay, and low prestige life of a grad student, but it's hard to let all that get you down in a place like this.

Of course, this is mostly the fault of my fellow grad students, without whom I would have gone entirely insane. Because I don't want this section to consist of half the length of my thesis, I will only mention a few people by name, though many more are deserving; I hope the current crop of grad students continue the supportive and social culture in the department. While this list is woefully incomplete, I submit: Greg Sivakoff, who was finishing when I began, but came back to make sure I made it through, for being an unwavering friend and mentor at all levels; Jake Simon, Jarron Leisenring, and Ori Fox, for managing to share a home with me and with whom I could suffer the various stages of grad life; and Genevieve de Messieres, Joleen (Miller) Carlberg, Howard Powell, Nicole Gugliucci, and Paul Ries, for making my time here 
feel like I was surrounded by an extended family throughout.

But I never would have met any of them, had it not been for Tom Statler at Ohio University, who whisked me away to the MDM Observatory on Kitt Peak during my freshman year of college. After introducing me to astronomy as a career, he encouraged me to pursue all the available opportunities, without which I could never have completed this document. I still try to follow his advice to this day. At Ohio University, in the Physics and Astronomy Department, Honors College, and beyond, there are too many additional people to thank, but they are appreciated. Along the same lines, I have to acknowledge the amazing faculty at the Springboro Public Schools and all the athletic coaches I've had along the way, who encouraged me from the very beginning to work hard and pursue excellence and/or science. In particular, I am lucky to have been a student of Mrs. Jenkins, Mrs. Scott, Mr. Parker, Mr. Riegel, and Mr. Fox.

Writing this, I am overwhelmed thinking back on all the individuals who played both small and large roles in getting me here. The most constant of those are Gail Zasowski, the shining light of my life these last 4 years, and my immediate family. Without Gail, I would almost certainly still be writing this document, would very possibly be jobless, and would absolutely be a less enriched and a just plain less happy person. She relieved so many little burdens, like organizing social activities, proofreading job applications too much and often too late at night, and cooking delicious meals, not to mention the many occasions she helped me through research problems at an almost doctoral level (keep at it, you're almost there!). Most importantly, she gave me a faith in myself that was sometimes hard to find on my own, that kept me going in difficult times, and that provided a center from which I could venture out and to which I could return. To be as lucky as I am to have Gail in my life must be 
at a minimum a $5 \sigma$ event, though I suspect the true significance is much greater.

Through it all, though, has been my uniquely wonderful family, specifically my sister, Dr. Kari Wik, whose lifelong friendship I already know will be the richest of my life, and my parents Donald and Charlotte Wik. They encouraged me to try new things when I didn't want to, keep at things when I thought I couldn't do them, and pursue things they knew I liked but didn't really understand. All parents want the best for their children, but it can be much harder for them to help their kids find the best path; in retrospect, I have to give my parents complete credit for the way they made it possible for me to get here. Any child would be lucky to have gotten half the love, attention, encouragement, and good guidance that I have received. As such, this work is dedicated, as it only could be, to them. 


\section{Table of contents}

$\begin{array}{ll}\text { Abstract } & \text { ii }\end{array}$

Acknowledgements iv

List of Figures $\quad$ xix

List of Tables $\quad$ xxi

1 General Introduction 1

1.1 Galaxy Clusters: An Overview . . . . . . . . . . . . . . . . . 1

1.2 The Intracluster Medium . . . . . . . . . . . . . . . . . . . . . 3

1.3 Clusters as Cosmological Probes . . . . . . . . . . . . . 6

1.3.1 X-ray Mass Proxies . . . . . . . . . . . . . . . 7

1.3.2 The Sunyaev-Zel'dovich Effect . . . . . . . . . . . . . . 8

1.3 .3 Caveats ...................... . . . . 9

1.4 Fully Characterizing the State of All Phases of the ICM . . . . . . . 11

1.4.1 The Nonthermal Phase of the ICM . . . . . . . . . . . . 11

1.4 .2 The Coma Cluster . . . . . . . . . . . . . . . . . . . 13

2 The Impact of Galaxy Cluster Mergers on Cosmological Parameter Estimation from Surveys of the Sunyaev-Zel'dovich Effect 15

2.1 Introduction . . . . . . . . . . . . . . . . . . . . 17

2.2 Merger Simulations . . . . . . . . . . . . . . . . . . . 21

2.2.1 Equilibrium $Y-M$ and $y_{\max }-M$ Relations . . . . . . . 22

2.2 .2 Merger Boosts to $Y$ and $y_{\max } \ldots \ldots \ldots \ldots . \ldots . \ldots 24$

2.2 .3 Evolution of $y_{\max }$ and $Y$ During Mergers . . . . . . . . 28

2.3 Merger Trees . . . . . . . . . . . . . . . . . . . . . . . 31

2.3.1 Merger Boost Histograms . . . . . . . . . . . . . . 33

2.3.2 Generalizing Merger Boosts for Arbitrary Mass Ratio and Impact Parameter . . . . . . . . . . . . . . . 36

2.3.3 Adding Boosts to Merger Trees . . . . . . . . . . . . . . 37

2.4 SZ versus Mass Correlation _. . . . . . . . . . . . . . . 37

2.5 Distribution Functions of $Y$ and $y_{\max } \ldots \ldots \ldots \ldots$ 
2.6 Determining Cosmological Parameters from the Merger Tree YFs and

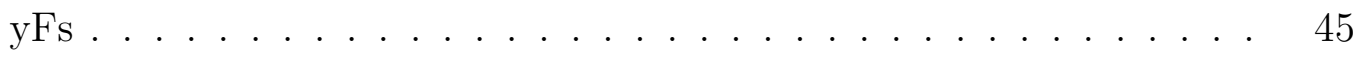

2.6.1 Varying only $\Omega_{M}$ and $\sigma_{8} \ldots \ldots$. . . . . . . . . . . . . . 46

2.6.2 Fitting the Dark Energy Equation of State Parameter $w$. . . 49

2.7 Discussion and Summary . . . . . . . . . . . . . . 52

3 A Suzaku Search for Nonthermal Emission at Hard X-ray Energies in the Coma Cluster $\quad 57$

3.1 Introduction . . . . . . . . . . . . . . . . . . . 58

3.2 Observations . . . . . . . . . . . . . . . . . . . . 61

3.3 Extraction and Construction of Spectra . . . . . . . . . . . 63

3.3.1 HXD-PIN Spectrum and Non-X-ray Background . . . . . . . . 63

3.3.2 XMM EPIC-pn Spectrum . . . . . . . . . . . . . . . 68

3.3.3 XMM EPIC-pn and Suzaku HXD-PIN Cross-Calibration . . . 74

3.3.4 Cosmic X-ray Background . . . . . . . . . . . . . . . 76

3.3.5 Point Sources . . . . . . . . . . . . . . . . . . . 79

3.4 Spectral Fits. . . . . . . . . . . . . . . . . . 80

3.4.1 Joint XMM-Newton and Suzaku Spectral Fits Without Considering Systematic Errors. . . . . . . . . . . . . 80

3.4.2 Multiple Thermal Components in Coma . . . . . . . . . . . . 82

3.4.3 Systematic Errors in the Spectrum . . . . . . . . . . . . . . 89

3.5 Implications and Discussion . . . . . . . . . . . . . . . . . . . 92

4 The Lack of Diffuse, Non-thermal Hard X-ray Emission in the Coma Cluster: The Swift BAT's Eye View $\quad \mathbf{1 0 0}$

4.1 Introduction . . . . . . . . . . . . . . . . . . 101

4.2 Observations . . . . . . . . . . . . . . . . . . 106

4.2.1 XMM-Newton EPIC-pn Mosaic Observations . . . . . . . . . . 106

4.2.2 The Swift BAT 58-Month Survey . . . . . . . . . . . . . . . . 108

4.3 Characterization of Extended Emission in BAT Images . . . . . . . . 112

4.3.1 Model Spatial Distributions of Hard X-ray Emission . . . . . . 112

4.3.2 Spatial Fits to the BAT data . . . . . . . . . . . . 116

4.4 Spectral Fits . . . . . . . . . . . . . . . . . . . . . . . . . . 119

4.4.1 Joint XMM-Newton EPIC-pn - Swift BAT Thermal Emission Fit122

4.4 .2 Nonthermal Spectra . . . . . . . . . . . . . . . . 126

4.5 Upper Limits to Diffuse, Nonthermal Emission . . . . . . . . . . . . . 128

4.6 Implications and Discussion . . . . . . . . . . . . . . . . . . 134

5 The Swift BAT Perspective on Nonthermal Emission in HIFLUGCS Galaxy Clusters 139

5.1 Introduction . . . . . . . . . . . . . . . . . . . . 140

5.2 Observations and Data Preparation . . . . . . . . . . . 143 
5.2.1 XMM-Newton EPIC Spectra . . . . . . . . . . . . . . 143

5.2.2 Swift BAT 58-month Survey Spectra . . . . . . . . . . . . . . 146

5.3 Separate Fits to Individual XMM-Newton EPIC and Swift BAT Spectra150

5.3.1 Single Temperature Fits to the EPIC Spectra . . . . . . . . . 150

5.3.2 Nonthermal Fits to the BAT Spectra . . . . . . . . . . . . . . 152

5.4 Joint Fits to the EPIC-BAT Spectra . . . . . . . . . . . . . . . 153

5.4.1 General Properties from the Joint Analysis . . . . . . . . . . . 155

5.4 .2 Individual Cases . . . . . . . . . . . . . . . . . . 157

5.4 .3 Upper Limits . . . . . . . . . . . . . . . . . . . . . . . . . . . 168

5.5 Joint Fits to Stacked EPIC-BAT Spectra . . . . . . . . . . . . . . . . 169

5.6 Implications and Discussion . . . . . . . . . . . . . . . 180

6 Summary and Future Prospects $\quad 211$

6.1 The Effect of Mergers on the SZ Properties of Clusters, and Cosmological Studies with SZ Cluster Samples . . . . . . . . . . . . . 211

6.2 IC Emission at Hard X-ray Energies . . . . . . . . . . . . . . . . . . . 213

6.2.1 IC Emission from the Coma Cluster . . . . . . . . . . . . . . . 214

6.2.2 IC Emission from the HIFLUGCS Cluster Sample . . . . . . . 214

6.2.3 Future Work on IC Emission . . . . . . . . . . . . . . . . . . 215

6.3 Low Frequency Synchrotron Emission . . . . . . . . . . . . . . . . . 217

6.4 Cosmic Rays in the ICM . . . . . . . . . . . . . . . . . . . . . 218

6.5 Future Observatories . . . . . . . . . . . . . . . . . . . . 219

\section{$\begin{array}{ll}\text { A Fitting Simulation Data } & 220\end{array}$}

B XMM-Newton EPIC-pn-Swift BAT Cross-Calibration 223

B.1 The Spectrum of the Crab According to XMM-Newton . . . . . . . . 224

B.2 The Hard X-ray Spectrum of the Crab . . . . . . . . . . . . . 226

C Extracting BAT Fluxes from Extended Sources 229

C.1 BAT Point Spread Function . . . . . . . . . . . . . . . . . . 229

C.2 Tests of the Detection of Extended Sources . . . . . . . . . . . . 231

D Uncertainties in BAT Fluxes $\quad 236$

D.1 Flux Uncertainties for Point Sources . . . . . . . . . . . . . 236

D.2 Flux Uncertainties for Extended Sources . . . . . . . . . . . . . 237 


\section{List of Figures}

2.1 Images of the SZ parameter $y$ from 3D snapshots of the 1:3 mass ratio, $2 r_{s}$ impact parameter merger simulation. Here $r_{s}$ is the NFW scale radius of the more massive cluster. Both images are viewed from a lineof-sight which is rotated $45^{\circ}$ from the merger axis and $45^{\circ}$ azimuthally from the merger plane. Left: 386 Myr before first core crossing. Right: 114 Myr after first core crossing. . . . . . . . . . . . . . . .

2.2 Evolution of the SZ effect during a merger. In each panel, different curves are for different values of the merger impact parameter: $b=0$ (solid, black line), $2 r_{s}$ (dashed,red line), and $5 r_{s}$ (dotted, blue line), where $r_{s}$ is the NFW scale radius of the more massive cluster. In the left panels, the $b=2 r_{s}$ simulation run is offset downward by 0.15 and the $5 r_{s}$ run is offset downward by 0.3 for clarity. The time is scaled by the sound crossing time $t_{\mathrm{sc}}$ of the more massive premerger cluster. Left: Integrated Comptonization parameter $Y$ versus time for the 1:1 (top), 1:3 (middle), and 1:6.5 (bottom) mass ratios. Right: Maximum Comptonization parameter $y_{\max }$ versus time for the 1:1 (top), 1:3 (middle), and 1:6.5 (bottom) mass ratios. The mergers are observed $90^{\circ}$ to the merger axis and in the merger plane. . . . . . . . . . . . .

2.3 Left: Histogram of the total time the integrated Comptonization parameter $Y$ is above some fraction of its initial premerger value $Y(0)$, scaled by the sound crossing time $t_{\mathrm{sc}}$ of the more massive premerger cluster. Histograms are shown for equal-mass mergers at 3 impact parameters $b=0$ (solid, black line), $2 r_{s}$ (dashed, red line), $5 r_{s}$ (dotted, blue line), where $r_{s}$ is the NFW scale radius of the more massive cluster. Right: Histogram of times for $y_{\max }$. . . . . . . . . . .

2.4 Integrated Comptonization parameter $Y$ (top panels) versus total mass in the flat cosmology at $z=0$ (left panels) and $z=1$ (right panels) for clusters with $Y>10^{-5} h^{-2} \mathrm{Mpc}^{2}$. The combined mass of both merging clusters is used if $Y$ is boosted or $t_{\text {obs }}>t_{\text {merge }}$, where $t_{\text {merge }}$ is the time of maximum boost. The apparent solid line is the result of many individual clusters at or near their equilibrium values of $Y$. In the bottom panels, the ratio of the boosted clusters to their equilibrium values for each redshift is shown. Each panel contains 5190 clusters. 
2.5 Same as Figure 2.4, but for the maximum Comptonization parameter $y_{\max }$, for clusters with $y_{\max }>10^{-5}$. Each panel contains 5663 clusters.

2.6 Boosted (dashed line) versus nonboosted (solid line) integrated Comptonization parameter function YF (left panel) and maximum Comptonization parameter function yF (right panel) histograms for $z=0$ (top, black), $z=0.5$ (middle, red), and $z=1$ (bottom, blue) in the flat universe. The smooth curves are the analytic PS predictions at each redshift given by equation (2.7). The residual plots give the difference in the logs between the boosted and nonboosted YFs and yFs. Note the significant difference in scales of the residuals between the YFs and

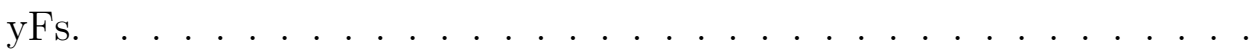

3.1 Suzaku HXD-PIN NXB model spectrum (red data points) compared to the Coma cluster data (uncorrected for background). Note that at energies above $45 \mathrm{keV}$, the NXB dominates the data and that deviations of the data above the NXB are confined to individual channels that are simply statistical fluctuations or are otherwise imperfectly characterized by the NXB model. . . . . . . . . . . . . . . . .

3.2 Suzaku HXD-PIN Earth-occulted data (black data points) compared to the NXB model spectrum for the same time periods of Earthoccultation (red data points). During Earth-occultation, the only events should be due to the NXB. Note that the NXB agrees well with the normalization and shape of the Earth-occulted data at all energies $\gtrsim 12 \mathrm{keV}$. (Only this range is used in the spectral fits for Coma.) . .

3.3 XMM EPIC-pn 2-7.5 keV wavelet smoothed X-ray surface brightness image (Schuecker et al. 2004) with contours of constant Suzaku HXDPIN effective area overlaid. The contours are spaced at $10 \%$ intervals running from $0 \%$ to $90 \%$ of the effective area for a point source located at the instrument center. In between the contours, the regions labeled R1, R2, etc., correspond to those in Table 3.1 and in the text. . . . .

3.4 Suzaku HXD-PIN spectrum $(E>12 \mathrm{keV})$ and the combined XMM spectrum $(E<12 \mathrm{keV})$ corresponding to the spatial sensitivity of the PIN. Shown as solid lines are the best fit models for a single temperature thermal component. The thermal model ("APEC", green) is nearly coincident with the data, though falling below it at higher energies. Also included for all joint fits are the the total spectrum for the "XMM Point Sources" (red) and the Cosmic X-ray Background ("CXB", purple), the latter of which only applies to the PIN spectrum since the CXB is subtracted from the $X M M$ data along with the NXB. 
3.5 Suzaku HXD-PIN spectrum $(E>12 \mathrm{keV})$ and the combined XMM spectrum $(E<12 \mathrm{keV})$ corresponding to the spatial sensitivity of the PIN. Shown as solid lines are the best fit models for a single temperature thermal component plus a nonthermal component. The thermal model ("APEC", green) is nearly coincident with the data, though falling below it at higher energies. The nonthermal model ("Power Law", light blue) is the faintest model component for both spectra, and the photon index is fixed at $\Gamma=2.0$. The other two components are described in Figure 3.4. . . . . . . . . . . . . . . . . . .

3.6 Suzaku HXD-PIN spectrum $(E>12 \mathrm{keV})$ and the combined $X M M$ spectrum $(E<12 \mathrm{keV})$ corresponding to the spatial sensitivity of the PIN. Shown as solid lines are the best fit models for a two-temperature thermal component. The thermal model ("APEC+APEC", green) is nearly coincident with the data, though falling below it at higher energies. The other two components are described in Figure 3.4. . . . .

3.7 XMM-Newton temperature map across Coma with HXD-PIN contours of constant PIN effective area overlaid at $10 \%$ intervals. The $X M M$ Newton spectra were fit in square spatial regions 4.3 on a side. The temperatures, given in $\mathrm{keV}$ by the color bar, are accurate to either a few tenths of a $\mathrm{keV}$ (in the center) or $1-2 \mathrm{keV}$ in lower surface brightness regions. Temperatures shown here were determined from fits to the 0.5-14 keV spectrum in each region. . . . . . . . . . . . . . .

3.8 Suzaku HXD-PIN spectrum $(E>12 \mathrm{keV})$ and the combined XMM spectrum $(E<12 \mathrm{keV})$ corresponding to the spatial sensitivity of the PIN. Shown as solid lines are the combined spectra of the best fit models from the temperature map. The thermal model ("Tmap", green) is nearly coincident with the data, though falling below it at higher energies. The other two components are described in Figure 3.4.

3.9 Suzaku HXD-PIN spectrum $(E>12 \mathrm{keV})$ and the combined XMM spectrum $(E<12 \mathrm{keV})$ corresponding to the spatial sensitivity of the PIN. The PIN NXB is increased to its $90 \%$ confidence limit, which is $2.3 \%$ for $E<40 \mathrm{keV}$ and $4 \%$ for $E>40 \mathrm{keV}$. The thermal model ("APEC", green) is nearly coincident with the data, though falling below it at higher energies. The other two components are described in Figure 3.4. Note that the residuals above the model that exist in the previous fits at $E>40 \mathrm{keV}$ have disappeared. . . . . . . . . . . . 
3.10 Suzaku HXD-PIN spectrum $(E>12 \mathrm{keV})$ and the combined $X M M$ spectrum $(E<12 \mathrm{keV})$ corresponding to the spatial sensitivity of the PIN. All quantities with systematic uncertainties (PIN CXB and NXB, the XMM-Suzaku cross calibration) are set to their $90 \%$ confidence limit in the direction that favors the addition of a power law model component to describe the data. The thermal model ("APEC", green) is nearly coincident with the data, though falling below it at higher energies. The nonthermal model ("Power Law", light blue) is shown for $\Gamma=2$ at its $90 \%$ confidence upper limit value. The other two components are described in Figure 3.4. . . . . . . . . . . . . .

4.1 XMM-Newton EPIC-pn 2-7.5 keV wavelet-smoothed X-ray surface brightness image (Schuecker et al. 2004) with contours from the raw Swift BAT $14-20 \mathrm{keV}$ survey image (square root spacing: $0.0,2.625 \times 10^{-6}$, $1.05 \times 10^{-5}, 2.3625 \times 10^{-5}$, and $4.2 \times 10^{-5}$ counts $\left.\mathrm{s}^{-1} \mathrm{pix}^{-1}\right)$. Negative contours are not shown for clarity, and note that the FWHM of the BAT PSF is 19.5. The (red) box shows the $65.5 \times 65.5$ region from which the EPIC-pn spectrum is extracted for joint fits. The BAT emission is slightly more extended to the W-SW, as would be expected from the higher temperature gas in that direction. Note, however, that the outermost contour is consistent with noise and should be ignored.

4.2 The radial profile of the Coma cluster (crosses, black) compared to a point source of comparable brightness (squares, red; scaled slightly to match Coma's central flux). Each point represents an individual pixel. The BAT emission from Coma is clearly extended and not axially symmetric, as shown by the the larger spread in pixel count rates in its profile compared to the point source. . . . . . . . . . . . . . .

4.3 Images from the 8 energy bands of the Swift BAT survey (first and third columns). The greyscale follows a square root scaling from 0 counts/s (white) to $\left[>4.2 \times 10^{-5}(\mathrm{E} 1),>1.7 \times 10^{-5}(\mathrm{E} 2-\mathrm{E} 3),>5 \times 10^{-6}(\mathrm{E} 4-\right.$ E8)] counts $\mathrm{s}^{-1} \mathrm{pix}^{-1}$ (black). The contours in the images in the first column show the best-fit thermal model for each band and follow a square root spacing from 0 to the maximum of the greyscale for that band, with 5 contours. For E4, only three contours are shown as the model is fainter than the brightest region of the data to the west of the cluster center. For E1, the contours occur at the same levels as shown in Fig. 4.1). The middle column shows the thermal model-subtracted residual images for E1-E4, with the same for greyscale as the data on the left. The residuals show that the thermal spatial models are generally well-mapped to the actual data. Note that the background is also fit for and subtracted from the data in the residual images, so the outer fluctuations are not identical to those in the left column. 
4.4 Radial profiles of the data and thermal model fits shown in the first column images of Fig. 4.3. BAT pixels are averaged in annuli of $1^{\prime}$ width (crosses), as are the model values for each pixel position (histogram); the residuals are plotted below each fit, on the same scale as the fit. The structure in the E1 and E3 residuals could be due to a slightly larger PSF FWHM and/or a true spatial distribution of emission that differs slightly from our models; in either case, the effect on the extracted flux would be less than its $1 \sigma$ error. . . . . . . . . . . . .

4.5 The top panel shows the BAT E1 profile and spatial fit for a model with both thermal emission and nonthermal emission following the $\mathrm{KW}$ model (histogram, red). The best-fitted value of the normalization of the KW component is actually negative. The dash-dot line represents the background level and the dashed line (blue) shows the predicted spatial distribution of flux for the KW model (Kushnir \& Waxman 2010) from 14-20 keV. The inset expands the scale of the y-axis above it to highlight the difference between the data and the expected flux. In the bottom panel, we perform the same fit to simulated BAT data based on the thermal plus KW model, including shot and systematic noise comparable to that present in the actual data. This shows that the BAT would have easily detected a nonthermal component with the spatial distribution given by the KW model and the predicted flux. .

4.6 Single temperature (APEC) fit to the XMM-Newton EPIC-pn and Swift BAT spectra. The BAT spectra shown are reduced to the fraction which occurs in the XMM-Newton spectral extraction region. The BAT spectra were constructed assuming the spatial distribution predicted by the XMM-Newton temperature map. The origin of the low E1 (14$20 \mathrm{keV}$ ) flux is discussed in the text. A single temperature model $(k T=8.24 \mathrm{keV})$ is sufficient to describe the $2-200 \mathrm{keV}$ emission from the central square degree region of the Coma cluster. . . . . . . . . .

4.7 Thermal plus nonthermal model fit to the Swift and XMM-Newton data for the $\Gamma=2$ power law nonthermal model corresponding to the $90 \%$ upper limit, including the systematic uncertainties as described in the text. This example, which is the model with most significant nonthermal flux, is for a $25^{\prime}$ radius, uniform surface brightness disk of nonthermal emission with a position offset from the center of the large-scale thermal emission by -2.5 and $10^{\prime}$ in $l$ and $b$, respectively. As is true of all the upper limits from the joint spectra, the nonthermal component does not exceed $\sim 1 \%$ of the low energy part of the $X M M$ Newton spectrum, nor does it compete with the thermal emission until energies $E \gtrsim 50 \mathrm{keV} \ldots \ldots \ldots \ldots$ 
4.8 Upper limits (small arrows) for each nonthermal spatial model relative to the RXTE (Rephaeli \& Gruber 2002, upper cross, green), Beppo$S A X$ (Fusco-Femiano et al. 2004, lower cross, red), and Suzaku (Wik et al. 2009, long arrow, blue) detections/upper limit. The previous flux detections $\left[(1.5 \pm 0.5) \times 10^{-11} \mathrm{ergs} / \mathrm{cm}^{2} / \mathrm{s}\right]$ and upper limit $\left(6 \times 10^{-12}\right.$ ergs $/ \mathrm{cm}^{2} / \mathrm{s}$ ) are corrected to account for the fraction of emission missed due to vignetting by the collimator response functions (see text). In the top panel, upper limits are calculated from the simultaneous joint fits to the XMM-Newton and Swift spectra, and all nonthermal fluxes reported are from inside the $X M M-N e w t o n$ extraction region (the square in Fig. 4.1). In the bottom panel, upper limits are derived from BAT spectra created from the nonthermal component of spatial fits only, and the fluxes represent the total emission of the spatial model. Based on the results presented in the top panel, we conclude that extended IC emission cannot reconcile the discrepancy between the Suzaku and RXTE/Beppo-SAX observations. . . . . . . . . . . . . .

5.1 Source extent as a function of distance from the Galactic center in the 14-20 keV BAT band. Filled circles (red) represent the mean FWHM of all non-cluster sources within a region of $30^{\circ}$ radius for various independent positions distributed evenly over the sky. The hatched area marks one standard deviation of the FWHM distribution for all noncluster sources. Note that the red circles indicate the average FWHM of several sources; consequently, their scatter about the mean FWHM value of all sources, indicated with the dashed line, will be reduced by the square root of the number of objects inside that region, i.e. the "error of the mean," which is why their scatter is less than that shown by the hatched area. Detected clusters are shown as triangles (green) and are circled if they have $\mathrm{S} / \mathrm{N}>10$. Many bright galaxy clusters are at least somewhat resolved by the BAT, which must be accounted for when extracting fluxes from the BAT survey. . . . . . . . . . . .

5.2 The distribution of power law normalizations (with a fixed photon index $\Gamma=2$ ) fit to 10,000 blank sky spectra extracted from the BAT survey (black histogram). The best-fit Gaussian distribution is overlaid as the smooth, dashed red line. In blue, similar best-fit normalizations are shown for the 58 HIFLUGCS clusters (see text for details), with individual normalizations represented as vertical lines. The cluster histogram has been scaled up to show its agreement with the blank sky spectra. In general, the cluster BAT spectra lack any clear evidence for a nonthermal component, except in a few cases comprising the positive tail of the blue histogram. . . . . . . . . . . . . . . 
5.3 A comparison of best-fit temperature values in $1 \mathrm{~T}$ fits to only the $X M M-N e w t o n$ spectra (x-axis) and to the EPIC and BAT spectra simultaneously (y-axis). Solid lines indicate the $90 \%$ error interval for $E>2 \mathrm{keV}$ fits, dashed (red) lines for $E>3 \mathrm{keV}$, and the dashed diagonal line represents equality between the two temperature determinations. Jointly fitting both datasets yields consistent temperatures to those derived only in the XMM-Newton band. Fitting over a slightly higher energy range ( $E>3 \mathrm{keV})$, while increasing the average temperature by $\sim 0.3 \mathrm{keV}$ in the EPIC bandpass (see text), does not increase the joint fit temperatures as much; note how the dashed points fall slightly below equality for moderately hot clusters. . . . . . . . . .

5.4 Upper limits and measurements of the nonthermal spectral component in the $3-195 \mathrm{keV}$ joint fits as a function of cluster temperature. Limits and error bars indicate the $90 \%$ confidence interval without considering the impact of systematic uncertainties. In general, an excess attributable to IC emission is not observed, and the few detections, discussed individually in the text, have marginal statistical significance. 159

5.5 Abell 2029: The T+IC model simultaneous fit to the EPIC $(E<12$ $\mathrm{keV})$ and $\operatorname{BAT}(E>14 \mathrm{keV})$ spectra. The EPIC-pn spectrum and residuals are in green, and the MOS 1 and 2 spectra/residuals are in dark and light blue, respectively. The like-colored lines below these spectra show the CXB model contribution. The total model fit and thermal contribution is represented by the black histogram, and the red lines represent the nonthermal $(\Gamma=2)$ spectral component. . .

5.6 Abell 1651: The T+IC model simultaneous fit to the EPIC $(E<12$ $\mathrm{keV})$ and $\operatorname{BAT}(E>14 \mathrm{keV})$ spectra. The notation is identical to Figure 5.5.

5.7 Abell 2142: The T+IC model simultaneous fit to the EPIC $(E<12$ $\mathrm{keV})$ and $\operatorname{BAT}(E>14 \mathrm{keV})$ spectra. The notation is identical to Figure 5.5.

5.8 Abell 3112: The T+IC model simultaneous fit to the EPIC $(E<12$ $\mathrm{keV})$ and $\operatorname{BAT}(E>14 \mathrm{keV})$ spectra. The notation is identical to Figure 5.5.

5.9 Abell 1367: The T+IC model simultaneous fit to the EPIC $(E<12$ $\mathrm{keV})$ and $\operatorname{BAT}(E>14 \mathrm{keV})$ spectra. The notation is identical to Figure 5.5. 
5.10 The two temperature values in the $2 \mathrm{~T}$ fits $(2-195 \mathrm{keV})$ with respect to the single temperature fit for each cluster over the same range. High (triangles, black) and low (circles, red) temperature values in the $2 \mathrm{~T}$ model for the same cluster are connected by dotted lines for clarity. Blue circles represent fits with an unphysical best-fit $2 \mathrm{~T}$ model, with either unrealistically high temperatures $(k T>14 \mathrm{keV}$, except for A0754) or a low temperature component that over-predicts the emission below $2 \mathrm{keV}$. (These have $k T \lesssim 2.1 \mathrm{keV}$ for clusters with single temperatures of $3.5 \mathrm{keV}$ or hotter.) These excluded regions are indicated by dashed lines in the figure, and the diagonal dashed line represents equality of $1 \mathrm{~T}$ and $2 \mathrm{~T}$ temperatures. These temperature values are used to build the $2 \mathrm{~T}_{\mathrm{J}}$ model used in fits to the stacked spectra. . . . . . . . . . . . . . . . . .

5.11 The stacked spectrum of all 58 clusters with the combined single temperature model fit $\left(1 \mathrm{~T}_{\mathrm{X},>2}\right)$. The EPIC-pn spectrum $(E<12 \mathrm{keV})$ and BAT spectrum $(E>14 \mathrm{keV})$ are shown in the top panel, and their residuals in the lower panel. The CXB contribution appears below the EPIC-pn data. The problems between 2-3 keV (described in the text) clearly show up in the residuals, as does a potential problem with low energy BAT fluxes. The combined single temperature model determined from the $2-12 \mathrm{keV}$ fits is sufficient to explain the summed BAT spectrum; no nonthermal excess is obvious.

5.12 The stacked spectrum of all clusters with large-scale, diffuse radio halos or relics. General features of the plot are the same as Figure 5.11. The red line represents the best-fit nonthermal model with photon index $\Gamma=2$, and the lower black line shows the $1 \mathrm{~T}_{\mathrm{X},>2}$ thermal model. . .

5.13 The stacked spectrum of all clusters with large-scale, diffuse radio halos or relics with the combined single temperature model fit $\left(1 \mathrm{~T}_{\mathrm{X},>2}\right)$. General features of the plot are the same as Figure 5.11. A slight excess is apparent in the BAT spectrum, due to either a nonthermal spectral component (see Fig. 5.12) or a significant multi-temperature structure in the individual clusters (see Fig. 5.14) . . . . . . . . . . . . .

5.14 The stacked spectrum of all clusters with large-scale, diffuse radio halos or relics with the combined double temperature model fit $\left(2 \mathrm{~T}_{\mathrm{J}}\right)$. General features of the plot are the same as Figure 5.11. The combined $2 \mathrm{~T}_{\mathrm{J}}$ can explain the slight excess seen when the single temperature model

5.15 The stacked spectrum of all clusters without diffuse radio emission shown with the combined single temperature model fit $\left(1 \mathrm{~T}_{\mathrm{X},>2}\right)$. General features of the plot are the same as Figure 5.11. . . . . . . . 
C.1 Fits to the BAT PSF. In the top and middle panels, the profiles of two point sources, the Crab and Cyg X-2, are fit to a Gaussian. Large, regular residuals (middle panels) remain, which are mostly removed (at the $<1 \%$ level, bottom panels) after modifying our expression for the PSF (Eqn. C.1). Lingering residuals, which are particularly significant for the Crab but only slightly noticeable for the other source, primarily result from spatial asymmetries due to the actual angular extent of the source, as in the case of the Crab, and the rectangular shape of the BAT instrument, which will cause off-axis sources to be more "squished" in one direction than another. In the latter case, the effect of summing many individual pointings with the detector in various orientations almost, but not entirely, removes this azimuthal component of the PSF shape. . . . . . . . . . . . . . . . . .

C.2 Recovered surface brightness for simulated emission from a uniform surface brightness disk of a given radius. Error bars indicate the statistical error of the mean on the disk surface brightness, though the spatial fluctuations in the sky reconstruction behind this error are due entirely to systematic effects; the "noise" at any position in these simulations is determined by the flux of all the other sources within the FOV, or in this case the other parts of the disk. The variation with radius is smooth instead of random due to the systematic origin of the fluctuations. For angular sizes of interest here, $<90^{\prime}$, the intrinsic uncertainty in the recovered flux of an extended source, due to coded mask imaging techniques, is at most a few percent (based on the size of the error bars). Also, there is no loss in sensitivity to diffuse emission; all of the input flux is recovered, albeit with less and less precision for larger sources.

D.1 The standard deviation of the best-fit normalization for 100 simulations of each spatial model considered in this work. Both photon noise (in the background and source flux) and systematic effects (the influence of other point sources in the FOV and the relative off-axis angle of all sources relative to the detector) are included. The model area is shown in terms of the equivalent number of PSF areas, $N_{\mathrm{PSF}}$. For the thermal spatial models, only the lowest energy band (E1: $14-20 \mathrm{keV})$ is plotted for clarity. The solid line represents the expected $\sqrt{N_{\mathrm{PSF}}}$ dependence of the error (Equation D.1). The simulated values of the errors for the extended models fall below this result, and possible explanations of this behavior are briefly discussed in the text. Note that for the thermal E1 model, the difference in the value of $N_{\mathrm{PSF}}$ shown here and in Table B.1 results from the difference between the survey PSF and the on-axis PSF used in these simulations. . . . . . . . . . . . . . . 239 


\section{List of Tables}

2.1 Cosmological Parameter Values Used to Create Merger Trees . . . . 21

2.2 Merger-Induced SZ-M Relations and Scatter . . . . . . . . . . . . . 41

2.3 Best-Fit Values for $\Omega_{M}$ and $\sigma_{8}$ for Three World Models . . . . . . . 48

2.4 Best-Fit Flat World Models with Constant $w \ldots \ldots 6$

$3.1 X M M$ Regions and Spectral Fits . . . . . . . . . . . . 73

3.2 Fits to Joint XMM-PIN spectra . . . . . . . . . . . . . . . 77

$3.390 \%$ Upper Limits on IC Flux . . . . . . . . . . . . . . . . . 94

4.1 Joint Fits to XMM-Newton and Swift Spectra . . . . . . . . . . . . . 124

$4.290 \%$ Flux Upper Limits (20-80 keV) for Nominal NT Position ${ }^{\mathrm{a}}$. . . . 138

5.1 XMM-Newton Observations, Regions, and Cluster Classes . . . . . 185

5.1 XMM-Newton Observations, Regions, and Cluster Classes . . . . . 186

5.1 XMM-Newton Observations, Regions, and Cluster Classes . . . . . 187

5.2 EPIC-only, Single Temperature Fit Parameters . . . . . . . . . . . . 188

5.2 EPIC-only, Single Temperature Fit Parameters . . . . . . . . . . . . 189

5.2 EPIC-only, Single Temperature Fit Parameters . . . . . . . . . . . . 190

5.2 EPIC-only, Single Temperature Fit Parameters . . . . . . . . . . . . 191

5.3 Joint Thermal and Nonthermal Fits to the EPIC and BAT Spectra

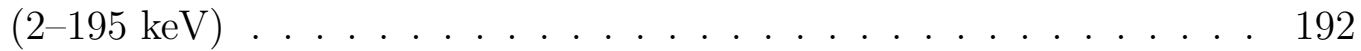

5.3 Joint Thermal and Nonthermal Fits to the EPIC and BAT Spectra

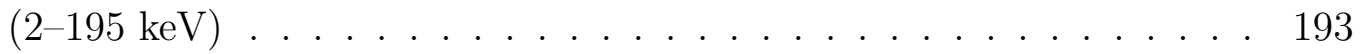

5.3 Joint Thermal and Nonthermal Fits to the EPIC and BAT Spectra

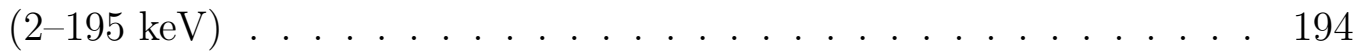

5.3 Joint Thermal and Nonthermal Fits to the EPIC and BAT Spectra

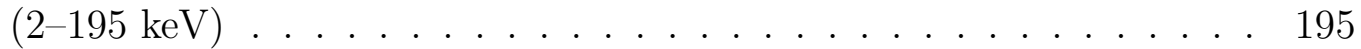

5.3 Joint Thermal and Nonthermal Fits to the EPIC and BAT Spectra $(2-195 \mathrm{keV}) \ldots \ldots \ldots \ldots . \ldots \ldots$

5.3 Joint Thermal and Nonthermal Fits to the EPIC and BAT Spectra

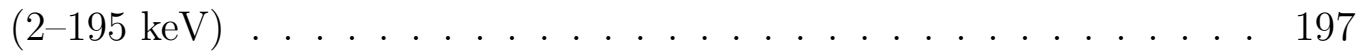

5.3 Joint Thermal and Nonthermal Fits to the EPIC and BAT Spectra

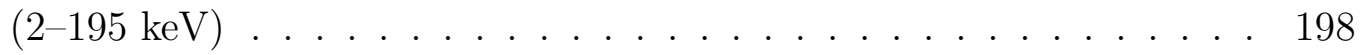


5.4 Joint Thermal and Nonthermal Fits to the EPIC and BAT Spectra

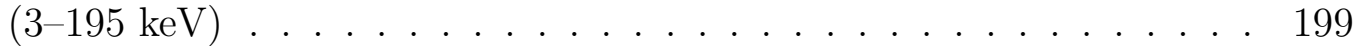

5.4 Joint Thermal and Nonthermal Fits to the EPIC and BAT Spectra

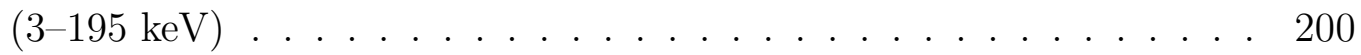

5.4 Joint Thermal and Nonthermal Fits to the EPIC and BAT Spectra

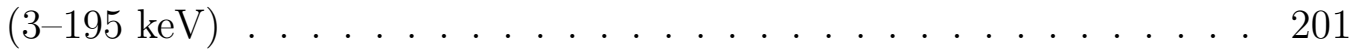

5.4 Joint Thermal and Nonthermal Fits to the EPIC and BAT Spectra

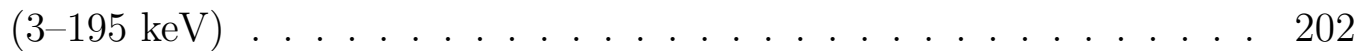

5.4 Joint Thermal and Nonthermal Fits to the EPIC and BAT Spectra

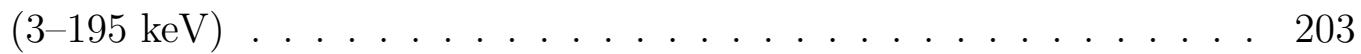

5.4 Joint Thermal and Nonthermal Fits to the EPIC and BAT Spectra $(3-195 \mathrm{keV})$. . . . . . . . . . . . . . . . . . . . 204

5.4 Joint Thermal and Nonthermal Fits to the EPIC and BAT Spectra

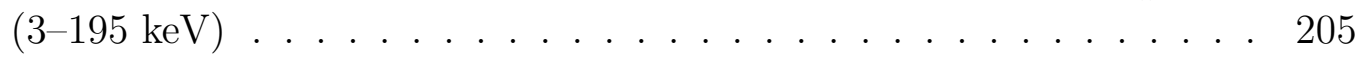

5.5 Upper Limits to 20-80 keV Nonthermal Flux from EPIC and BAT Joint Fits . . . . . . . . . . . . . . . . . . . . . . . . 206

5.5 Upper Limits to 20-80 keV Nonthermal Flux from EPIC and BAT Joint Fits . . . . . . . . . . . . . . . . . . . . . . . . . . . . . . 207

5.5 Upper Limits to 20-80 keV Nonthermal Flux from EPIC and BAT Joint Fits . . . . . . . . . . . . . . . . . . . . . . . . . . . . . 208

5.6 Fits to Stacked EPIC and BAT Spectra . . . . . . . . . . . . 209

5.6 Fits to Stacked EPIC and BAT Spectra . . . . . . . . . . . . 210

A.1 Fitting Parameters for Merger Boost Histograms . . . . . . . . . . . 222

B.1 Swift BAT Error Factors . . . . . . . . . . . . . . . . . 227 


\section{Chapter 1}

\section{General Introduction}

\subsection{Galaxy Clusters: An Overview}

Clusters of galaxies are the largest virialized structures in the universe, concentrating $10^{14}-10^{15} M_{\odot}$ of matter into $\sim \mathrm{Mpc}^{3}$ volume (Sarazin 1988). These scales ensure that, in a largely homogeneous universe, their contents are representative of the constituents of the universe as a whole, and that they will be noticed. First studied systematically over a century ago (Wolf 1906), clusters were originally recognized as a local clustering together of galaxies on the sky; though the true nature of the galaxies that made them up would not be uncovered for another two decades (Hubble 1925). Abell (1958) constructed the first comprehensive catalog, which classified clusters according to the number of bright galaxies they contained, and it was later extended to cover the southern sky as well (Abell et al. 1989). Except for a handful of clusters named after the constellations in which they reside, the majority of low redshift, well-studied clusters are known by this catalog number.

In the generic picture of the formation of structure in the universe, primordial density fluctuations in the otherwise uniform distribution of matter and energy - 
perhaps due to the amplification of quantum fluctuations during an early inflationary epoch - seed over-densities that grow from the accretion of surrounding material (e.g., Peebles 1993). The number density per unit mass, called the mass function, of the resulting largest fluctuations - galaxy clusters - is sensitive to the average matter density of the universe, $\Omega_{M}$, and the normalization of the power spectrum of initial fluctuations, usually denoted by $\sigma_{8}$ (Henry \& Arnaud 1991). The parameter $\sigma_{8}$ is formally the root mean square (rms) of the average density within spheres of radius $8 \mathrm{Mpc}$. How the mass function evolves, or the manner in which mass is accumulated into large and small structures alike, depends both on these and other cosmological parameters and on the dynamical state of the matter driving their formation. Our universe is most consistent with a dominant population of mass-carrying particles that are initially dynamically "cold," or non-relativistic. For negative power spectrum indices, larger scale fluctuations are outnumbered by smaller scale ones, which cannot be smoothed out by low kinetic energy particles; smaller perturbations therefore collapse and virialize first owing to their shorter dynamical times, and only later undergo mergers following the large scale gravitational field (Navarro et al. 1995). This scenario implies that clusters form primarily from merger and accretion processes, with the most massive clusters forming at the latest times. As galaxy groups fall in, they will remain coherent substructures for some period before being undone by phase mixing and minor interactions with cluster galaxies, which are routinely observed (e.g., Adami et al. 2005).

These dynamic, constantly evolving environments host more than just their constituent namesake, however. As has long been known (Zwicky 1933), galaxies contain far too little mass to account for their observed velocity dispersion, which directly relates to the total enclosed mass of the system. Most of this unseen mass takes the 
form of an as-yet unidentified type of non-baryonic matter, dubbed dark matter, but not all. Some tailed radio galaxies in clusters exhibit kinks or bends in the jets producing them, as if affected by an intergalactic wind. It was soon demonstrated that the movement of these galaxies through a stationary "intracluster medium" (ICM) of sufficient density could provide enough ram pressure to cause the observed deflections (Begelman et al. 1979). In fact, while dark matter dominates overall, this medium outweighs its companion galaxies by a factor of 5 to 6 (e.g., White et al. 1993; LaRoque et al. 2006). Clearly, calling these structures "galaxy clusters" when galaxies make up such a small fraction of their content is something of a misnomer, not unlike nearly every other term in the astronomical lexicon!

\subsection{The Intracluster Medium}

While some baryonic matter falls into the gravitational potentials created by dark matter over-densities, eventually becoming the galaxies of today, most of the gas remains between galaxies. Gas near the dark matter halos hosting galaxy clusters will quickly have their recession velocity, due to the Big Bang expansion, be overcome by the nearby, deep potential well. In the idealized, spherically symmetric scenario, gas at increasingly large radial distances will turn around and fall into the potential. The infall velocity generally exceeds the sound speed of the gas, leading to internal shocks that can thermalize its kinetic energy and an accretion shock that propagates out as more gas accretes onto the cluster potential (Gunn \& Gott 1972; Bertschinger 1985). Although the expectation was that this gas would be shock-heated to a temperature resulting in rough hydrostatic equilibrium (HSE) with the gravitational potential, which in massive clusters leads to a temperature of $10^{8} \mathrm{~K}$, the first truly diffuse emission associated with the ICM was observed in the radio. Willson (1970) detected 
extended, nonthermal synchrotron radiation in the Coma cluster, which results when a population of relativistic electrons spiral around magnetic field lines. Since it was long postulated that collisionless, magnetized shocks could accelerate cosmic rays in our Galaxy (Fermi 1949), the same process could be accelerating electrons across accretion shocks in clusters. How significant this population is, however, depends on the exact mechanism and efficiency of particle acceleration in these environments - which remains uncertain to this day (see Petrosian \& Bykov 2008, for a review). Whether the ICM is mostly in a thermal or nonthermal state must be addressed in the X-ray regime, where hot thermal electrons radiate via free-free (thermal bremsstrahlung) and collisionally excited line emission (Felten et al. 1966). A significant nonthermal electron population would also be detectable at X-ray energies, through inverse Compton (IC) interactions with cosmic microwave background (CMB) photons that up-scatter the CMB radiation to much higher energies (e.g., Rephaeli 1977).

Sounding rockets led to the first detection of X-ray emission from a galaxy cluster - or any extragalactic source - in the direction of of the Virgo cluster (Byram et al. 1966; Bradt et al. 1967). The first X-ray satellites allowed the entire sky to be surveyed, which showed that clusters were generally associated with high energy emission (Giacconi et al. 1972). Even in the early stages of opening up the X-ray spectral regime to observers for the first time, the emission was seen to be extended (Kellogg et al. 1972; Forman et al. 1972), though it could not be discerned from a population of discrete point sources, which might reside in the constituent galaxies. However, a thermal origin for the detected radiation was quickly recognized as a likely source (Cavaliere et al. 1971). While coarse spectra from these observations were most consistent with bremsstrahlung emission, the first definitive evidence came from the detection of highly ionized iron line emission, which clearly demonstrated the thermal 
nature of the gas (Mitchell et al. 1976). Today, far more sensitive instruments have led to the development of detailed emission models (e.g., APEC Smith et al. 2001) based on the most up-to-date atomic physics, which provide excellent descriptions of cluster data in general.

The recent generations of X-ray observatories, including Chandra, XMM-Newton, Suzaku, ASCA, ROSAT, and Beppo-SAX, have made possible the discovery of a wide range of phenomena occurring in the thermal phase of the ICM. Increases in spatial resolution have particularly expanded our understanding of the distribution of gas, and improving spectral resolution has allowed its thermodynamical state to be thoroughly characterized (Mushotzky 2004). Major and minor cluster mergers can produce severely disturbed ICM morphologies, which often include surface brightness discontinuities that can be identified as shocks and cold fronts through their temperature and pressure variations (Markevitch \& Vikhlinin 2007). Even in largely regular, relaxed clusters, distinct features have revealed themselves. In some such clusters, the supermassive black hole in the central galaxy drives powerful jets into the ICM, effectively blowing bubbles that displace the X-ray emitting gas (McNamara \& Nulsen 2007).

However, most clusters are found to be fairly regular, especially outside their core regions, following self-similarity, i.e. that more massive clusters look like scaled up versions of lower mass clusters. This property, along with the fact that the gas generally follows HSE with its gravitational potential, has meant that accurate cluster masses can be derived from simple measurements of the state of the ICM, such as its temperature or luminosity. If the masses of a large number of clusters in a given volume can be estimated, the cluster mass function can be determined, which as we have already seen is sensitive to the underlying cosmology (Voit 2005). With 
the discovery that the universe is not only expanding, but presently accelerating (e.g. Perlmutter et al. 1999), there is great interest in precisely determining the parameters that govern the contents and evolution of the universe as a whole.

\subsection{Clusters as Cosmological Probes}

The great advantage of using the cluster mass function comes from the exponential drop in the number of clusters with the highest masses (Press \& Schechter 1974). Therefore, small numbers of high mass - and typically bright - clusters are sufficient to constrain certain cosmological parameters; the first indication that $\Omega_{M}$ was not unity, as expected for the flat Einstein-deSitter geometry, came from early assessments of cluster abundances (Henry \& Arnaud 1991) and an assessment of the total amount of dark matter in the universe assuming that clusters are a fair sample of their contents (White et al. 1993). Since the turn of the millennium, one of the most mysterious features of the universe is that its expansion is accelerating, which has been generically dubbed dark energy and is quantified by $\Omega_{\Lambda}$. Its nature as a substance is completely unknown, although it could simply be a constant term $(\Lambda)$ in Einstein's equations of general relativity (Einstein 1917). However, the value of $\Omega_{\Lambda}$ and its evolution over time must first be constrained before theories describing it can be evaluated. Because it has emerged as a dynamical driver of structure formation relatively recently within the last half of the age of the universe - which coincides with the formation of massive clusters, the evolution of the mass function over this time is particularly sensitive to the evolution of dark energy (Haiman et al. 2001). To realize this goal, clusters must be detected through some observable property of their ICM, which can be used to infer their masses, in a survey spanning a large volume of space. Useful observables are typically found at either X-ray or microwave frequencies. 


\subsubsection{X-ray Mass Proxies}

Because thermal X-ray emission depends on the density squared, X-ray observations are biased by the densest regions of the ICM. These central regions also tend to be the most variable in cluster atmospheres, containing differing amounts of dense, cool (and thus bright) gas. Even so, robust trends exist between mass and observable X-ray quantities, generally referred to as scaling relations. The scatter about these relations will reduce the precision to which the cluster mass function can be measured, but as long as they are not biased systematically, cosmological parameter estimates will be accurate given enough data. The simplest quantity to relate to mass is the X-ray luminosity, which at least for the most massive and hot clusters can act as a proxy for cluster mass (Mantz et al. 2008). Another, more hydrodynamically-based quantity is the X-ray temperature $T_{X}$, since hotter clusters are generally more massive, but it suffers the drawback of requiring enough counts at harder $(>2 \mathrm{keV})$ energies to spectroscopically measure $T_{X}$. Numerical simulations show that the gas mass or the product of the gas mass with $T_{X}\left(Y_{X}\right)$ exhibit less scatter about the nominal relation (Kravtsov et al. 2006) since these are less biased by the central regions of the cluster. The usefulness of these X-ray mass proxies for constructing cluster mass functions and estimating cosmological parameters, including the equation of state of dark energy, has recently been demonstrated (Vikhlinin et al. 2009).

It should be noted that the total cluster mass is not a well-defined quantity itself. In practice, the mass must be defined within some radius, usually dependent on the sensitivity of the instrument employed. Generally, the larger the radius out to which the mass proxy is measured, the less scatter there is about a given scaling relation, since gas at larger radii is less affected by localized activity in cluster centers. On the other hand, the thermal pressure at large radii may be supplemented by bulk or 
turbulent motions (Lau et al. 2009) or simply underestimated if the electrons are not in equipartition (Wong \& Sarazin 2009).

\subsubsection{The Sunyaev-Zel'dovich Effect}

Another convenient observable of the ICM is known as the Sunyaev-Zel'dovich (SZ) effect (Sunyaev \& Zel'dovich 1972; Birkinshaw 1999). Any low frequency photons traversing the hot gas will have some probability of being slightly up-scattered via inverse Compton interactions; the dominant source of such photons are from the ubiquitous CMB. The SZ effect describes the distortion of the CMB spectrum due to this process, which can then be detected either individually in clusters or collectively in the CMB power-spectrum. The SZ effect produces a decrement (increment) in CMB surface brightness towards a cluster at low (high) frequencies. The change in the CMB surface brightness depends on the Compton parameter $y$, which is proportional to the integral of the electron pressure along the line of sight:

$$
y \equiv \frac{\sigma_{T} k}{m_{e} c^{2}} \int n_{e} T_{e} d l \propto \int P_{e} d l
$$

Here, $\sigma_{T}$ is the Thomson cross-section of the electron, $n_{e}$ is the electron number density, $T_{e}$ is the electron temperature, $P_{e}$ is the electron pressure, and $l$ is the distance along the line of sight. Since HSE determines the variation of pressure as a function of radius, $y$ can lead to several natural proxies for cluster mass. Perhaps most useful is the integral of $y$ over the entire solid angle of the cluster to yield the integrated Compton parameter

$$
Y \equiv \int y d A=d_{A}^{2} \int y d \Omega \propto \int P_{e} d V
$$


where $A$ is the projected area of the cluster, $\Omega$ is the solid angle, and $V$ is the cluster volume. Clearly, $Y$ measures the total thermal energy content of electrons in the ICM, as does $Y_{X}$ (which is modeled after $Y$ ). An essential advantage the SZ effect

has over many X-ray proxies is that it is proportional to $n_{e}$, not $n_{e}^{2}$, and so can be more easily observed out to larger radii where the more complex physical processes in the core are absent.

Perhaps the most important property of the SZ effect in practice, given that large surveys of clusters are needed to strongly constrain cosmological parameters, is that it is essentially redshift and distance independent. This aspect, thanks to the increasing temperature of the CMB with redshift, conveniently translates a flux-limited survey into a mass-limited survey, which allows the evolution of the mass function to be measured over the entire history of the universe during which clusters above a given mass have existed (Carlstrom et al. 2000). The leverage this feature gives to the study of evolving dark energy is crucial, not to mention the possibility that large numbers of clusters can be detected in unbiasedly, uniformly-performed, blind surveys. The main difficulty has been building telescopes sensitive enough to reliably observe this effect in any but the most massive galaxy clusters, which has only recently been achieved (Staniszewski et al. 2009).

\subsubsection{Caveats}

Given the great promise of using clusters to probe cosmological parameter space, it is critical to test whether these various mass proxies are in any way biased. The most significant difficulty for surveys is precisely what makes them particularly sensitive: the most massive clusters are also the likeliest to be undergoing a major merger. Almost by definition, massive clusters have had to undergo more merger events, and 
the fact that they tend to be located nearer other clusters exacerbates this problem (Sheth \& Tormen 1999). Mergers temporarily disrupt the equilibrium state of the entire ICM, thereby affecting the assumption of HSE. Undoubtedly, mergers will cause the scatter around scaling relations to be increased, but more worrisome is any systematic bias in the direction of the scatter. Just such a bias has been seen in numerical simulations of mergers of comparably sized clusters; both the X-ray luminosity and temperature are transiently boosted by up to a factor of up to 10 (Ricker \& Sarazin 2001; Ritchie \& Thomas 2002). While only a small number of clusters in a survey will be caught during such an event, the relatively small number of clusters at these high masses means that even rare events can significantly modify the mass function and lead to biased cosmological parameters, on the order of several tens of percent (Randall et al. 2002).

In contrast, SZ observations might be thought to be more robust to mergers, since they are less biased by the most central, densest gas. We explicitly test this expectation in detail in Chapter 2 and find that as long as $Y$ is used as the mass proxy, no significant distortion to the mass function occurs due to boosted values of $Y$ induced by mergers. For all merger geometries, the transient boost is always less than the increase to the final value of $Y$ in the newly merged cluster. Roughly, this result can be understood as a simple consequence of conservation of energy. During a merger, the kinetic energy of the infalling clusters is converted into thermal energy in the ICM. Since $Y$ represents the total electron thermal energy in the ICM, its value during a merger should generally lie between the values given by the two initial unmerged subclusters and the final value when essentially all of the merger kinetic energy has been thermalized. These results indicate that the thermal state of the ICM can be used to obtain accurate cluster masses, assuming the thermal gas dominates 
the energy budget of the ICM. While it certainly constitutes $\gtrsim 90 \%$ of the total energy (e.g., Vanderlinde et al. 2010), other percent-level components or phases of the ICM need to be included in the equations of HSE, which can only be possible once their own energy content is accurately determined.

\subsection{Fully Characterizing the State of All Phases of the ICM}

The observable properties of the ICM discussed thus far are all determined by the thermal gas, whose emission dominates at soft X-ray and microwave frequencies. However, any nonthermal constituent of the ICM will contribute to its pressure, and since it is the total (not just thermal) pressure that must be balanced by gravity due to the total mass of the cluster under HSE. Thus, cluster masses can only be accurately determined (at the percent level) if all pressure components have been correctly included.

\subsubsection{The Nonthermal Phase of the ICM}

As previously noted, the first definitive detection of emission from the ICM was actually of a nonthermal origin. This nonthermal phase, made up of relativistic electrons and ions, and magnetic fields, reveals itself in a relatively small fraction of massive clusters through structures called radio halos and relics. Radio halos span Mpc length scales and generally follow the thermal X-ray emission, while radio relics span similar scales but lie in the cluster outskirts. Both phenomena demonstrate the existence of a large-scale nonthermal phase of the ICM, but unfortunately its energetic importance remains unclear since neither the relativistic electron population density 
nor the magnetic field strength $B$ can be independently determined. The magnetic field strength, in particular, could contain a dynamically relevant amount of energy if it is at least as high as estimated from Faraday rotation measure (RM) studies, which probes the electron density-weighted $B$ field strength along a given line of sight through the ICM. The only way to determine the average value of $B$, and thus its energetic significance, is to definitively detect the associated IC emission.

Unfortunately, IC searches have been hampered by the lower sensitivity of instruments operating at higher X-ray energies, where the thermal emission exponentially declines and the IC signal becomes a more significant fraction of the total emission. Even so, telescopes with hard X-ray detectors onboard, such as Beppo-SAX and $R X T E$ have led to IC detections in several clusters, although of only marginal significance (e.g., Nevalainen et al. 2004). These detections need to be confirmed and the IC fluxes need to better constrained if the nonthermal phase is to be adequately characterized. In Chapter 5, we systematically search for IC emission from a sample of the brightest X-ray clusters using the Swift BAT all sky survey. We are unable to detect a significant nonthermal excess in any of the clusters, although marginal evidence for an excess above the thermal emission is seen in the stacked spectra from clusters hosting radio halos and/or relics. However, we still lack the sensitivity to rule out energetically significant nonthermal components.

Similarly, the cosmic ray population in clusters is currently only weakly constrained. While not addressed in this work, these relativistic hadron populations should be detectable in the gamma ray regime in the coming years. At GeV energies, the Fermi Gamma Ray Observatory is poised to either detect predicted populations, which may have some dynamical significance, or rule them out Ackermann et al. (2010). The TeV gamma ray flux from clusters is also beginning to constrain the dy- 
namical importance of very high energy cosmic rays (e.g., Aharonian et al. 2009a,b).

\subsubsection{The Coma Cluster}

If IC emission has truly been detected with current sensitivities, then the implied magnetic field strengths are too low to contribute any noticeable pressure to the ICM that might affect its structure. It is then crucial to corroborate these measurements with data from other instruments, so we can be assured that at least this aspect of the ICM does not need to be included in the equations of HSE. We therefore conduct a careful study of the hard X-ray emission from probably the most well-studied of all galaxy clusters, the Coma cluster. In the last decade, both $R X T E$ (Rephaeli \& Gruber 2002) and Beppo-SAX (Fusco-Femiano et al. 2004) observations suggested a significant nonthermal excess was present in Coma, though these results have been called into question (Rossetti \& Molendi 2004). Since then, two new observatories with instruments capable of confirming these detections have been launched: Suzaku and Swift. In Chapter 3, we discuss the analysis of the Suzaku HXD-PIN data and find an upper limit 2.5 times below the previously claimed detections. The smaller field of view of the HXD instrument, however, left open the possibility that our nondetection was a result of the emission being extremely extended far beyond the size of the observed radio halo. To test this possibility, we searched for extended nonthermal emission in the Swift BAT survey data on Coma, but observed no emission beyond that expected from the thermal gas. This analysis, presented in Chapter 4, finds upper limits to IC emission clearly below that previously claimed.

Thus, we have still failed to find any very significant detections of IC from clusters. However, the path forward, given upcoming X-ray missions and improvements in currently available data sets, is fairly clear. We complete this dissertation with a look 
to future work in this direction in Chapter 6. 


\section{Chapter 2}

\section{The Impact of Galaxy Cluster}

Mergers on Cosmological

Parameter Estimation from

Surveys of the Sunyaev-Zel'dovich Effect $^{1}$

\section{Abstract}

Sensitive surveys of the Cosmic Microwave Background (CMB) will detect thousands of galaxy clusters via the Sunyaev-Zel'dovich (SZ) effect. Two SZ observables, the central or maximum and integrated Comptonization parameters $y_{\max }$ and $Y$, relate in a simple way to the total cluster mass, which allow the construction of mass functions (MFs) that can be used to estimate cosmological parameters such as the ratio of the

\footnotetext{
${ }^{1}$ Published: Wik, D. R., Sarazin, C. L., Ricker, P. M., \& Randall, S. W. 2008, ApJ, 680, 17
} 
average matter density to the critical density $\Omega_{M}$, the normalization of the spectrum of initial density perturbations $\sigma_{8}$, and the dark energy equation of state parameter $w$. However, clusters form from the mergers of smaller structures, events that can disrupt the equilibrium of intracluster gas upon which $\mathrm{SZ}-M$ relations rely. From a set of N-body/hydrodynamical simulations of binary cluster mergers, we calculate the evolution of $Y$ and $y_{\max }$ over the course of merger events and find that both parameters are transiently "boosted," primarily during the first core passage. We then use a semi-analytic technique developed by Randall et al. (2002) to estimate the effect of merger boosts on the distribution functions $\mathrm{YF}$ and $\mathrm{yF}$ of $Y$ and $y_{\max }$, respectively, via cluster merger histories determined from extended Press-Schechter (PS) merger trees. The scatter in the $Y-M$ and $y_{\max }-M$ relations from merger boosts are found to be $\sim 2 \%$ and $25-30 \%$ respectively. To determine $\Omega_{M}, \sigma_{8}$, and $w$, the boosted and nonboosted YFs and yFs are fit with analytic PS distributions as a function of redshift. We find that boosts do not induce an overall systematic effect on YFs, and the values of $\Omega_{M}, \sigma_{8}$, and $w$ (assumed constant) were returned to within $2 \%$ of values expected from the nonboosted YFs. The boosted yFs are significantly biased, however, causing $\Omega_{M}$ to be underestimated by $15-45 \%, \sigma_{8}$ to be overestimated by $10-25 \%$, and $w$ to be pushed to more negative values by $25-45 \%$. We also fit YF as a function of redshift to cosmological models in which the dark energy parameter $w$ varied with redshift to assess the effects of mergers on the inferred change in $w$ with redshift. The values of $\Omega_{M}, \sigma_{8}$, and the low-redshift value of $w\left(w_{0}\right)$ were again reproduced to within $2 \%$. For the largest change in $w$ with $z$, which occurred between $z=0$ and $z=1$ for the models assumed, it was increased by about 0.04. Although this is twice as large as the merger effect on a constant value of $w$, it is still reasonably modest. We confirm that the integrated SZ effect, $Y$, is far more robust to mergers 
than $y_{\max }$, as previously reported by Motl et al. (2005) and similarly found for the X-ray equivalent $Y_{X}$ (Kravtsov et al. 2006; Poole et al. 2007), and we conclude that $Y$ is the superior choice for a mass proxy when using SZ observations of galaxy clusters to constrain cosmological parameters.

\section{$2.1 \quad$ Introduction}

The evolution of galaxy cluster abundance traces the massive end of the spectrum of initial density fluctuations and therefore is sensitive to cosmological parameters such as the ratio of the average matter density to the critical density $\Omega_{M} \equiv 8 \pi G \bar{\rho} /\left(3 H_{0}^{2}\right)$, the normalization of the power spectrum of initial density fluctuations $\sigma_{8}$, and the dark energy equation of state parameter $w$, equal to the ratio of the pressure to the energy density of dark energy. Here, $H_{0}$ is the Hubble constant and $\bar{\rho}$ is the average density in the universe. This sensitivity exists due to an exponential turnover at high masses in the mass function (MF) of clusters, which can be predicted from a well-established theoretical framework (e.g. Henry \& Arnaud 1991; Kitayama \& Suto 1996; Haiman et al. 2001). However, only gravitational lensing, which remains observationally challenging, directly measures the total mass of clusters. In order to get masses for the large number of clusters needed to construct the MF, it is often necessary to use a more observationally accessible quantity, such as the temperature or luminosity of X-ray emitting intracluster gas, from which the mass can be determined via some physical model. Relations between cluster mass and such a proxy typically require the gas to be in virial equilibrium; however, many processes are known that can disrupt the gas, including cluster mergers (Ricker \& Sarazin 2001, hereafter RS; Ritchie \& Thomas 2002; Poole et al. 2006) and AGN jet-blown radio bubbles (McNamara et al. 2005). 
There are many ongoing and planned surveys of clusters using the SunyaevZel'dovich (SZ) effect (Sunyaev \& Zel'dovich 1972; Birkinshaw 1999), which has the advantage of being effectively redshift-independent. The SZ effect is proportional to the integral of the electron pressure along the line of sight and can be characterized by the Comptonization parameter

$$
y \equiv \frac{\sigma_{T} k_{B}}{m_{e} c^{2}} \int n_{e} T_{e} d l \propto \int P_{e} d l
$$

where $n_{e}$ is the electron number density, $T_{e}$ is the electron temperature, $P_{e}$ is the electron pressure, and $l$ is the distance along the line of sight. The actual SZ flux, measured as a decrement or increment in the Cosmic Microwave Background (CMB), depends on frequency and is subject to relativistic effects for high temperature plasmas (for a review see Rephaeli 1995). Because we do not want to tie our results to any particular observational project, we use the frequency-independent Comptonization parameter in the following study, as has been standard in the literature. Also, we ignore any relativistic corrections as they are only relevant for the most massive clusters and because they modify $y$ in a complicated way that depends on frequency.

In general, SZ observations will give an image of the SZ effect or $y$ across the cluster. While specific values of $y$, for example the central or maximum value for a cluster (hereafter $y_{\max }$ ), are not expected to be a particularly robust proxy for the mass, the integrated Comptonization parameter $Y$ displays a tighter correlation with mass (Reid \& Spergel 2006). This is defined as

$$
Y=\int y d A=d_{\mathrm{A}}^{2} \int y d \Omega
$$

where $A$ is the projected surface area of the cluster on the sky, $\Omega$ is the solid angle, 
$d_{\mathrm{A}}$ is the angular diameter distance to the cluster, and the integral is over the entire cluster on the sky. Because the integrated Comptonization parameter is a global quantity, proportional to $\int P_{e} d V$ or the thermal energy content of the electrons, it should be less sensitive to non-equilibrium processes, which tend to be more localized in cluster cores. The usefulness of SZ surveys to constrain cosmological parameters has already been discussed extensively (e.g., Carlstrom et al. 2002; Haiman et al. 2001; Holder et al. 2001).

As with X-ray proxies for mass, the regularity of an $\mathrm{SZ}-M$ correlation relies on the fact that many clusters are energetically close to equilibrium. However, dynamically unrelaxed clusters should add scatter to this correlation. One mechanism known to disrupt the gas is cluster mergers, a direct consequence of hierarchical structure formation. How mergers affect the state of the gas will depend on the details of the individual mergers and their frequency, both of which depend on the cosmological model. To assess the utility of a mass proxy, such as the SZ effect, we need to quantify how mergers will affect the observed MF and consequently the estimation of cosmological parameters.

Current cosmological simulations, which accurately trace the collapse of structure and thus the merger history of clusters, cannot yet build the large samples of clusters at sufficient numerical resolution to constrain fundamental parameters and assess any potential bias due to mergers - though this approach is becoming viable (e.g., Hallman et al. 2007). Typically, N-body cosmological simulations of dark matter are re-simulated to include various types of gas processes such as "preheating," radiative cooling, and AGN feedback, from which the scatter to an observed SZ- $M$ correlation can be estimated. Depending on the resolution of the re-simulated hydrodynamic grid, these studies produce samples of $\sim 10$ (Nagai 2006; Bonaldi et al. 2007) to 100 (Motl 
et al. 2005; da Silva et al. 2004) clusters. Based on similar samples of simulations, Kravtsov et al. (2006) have defined an SZ-like X-ray observable, $Y_{X}$, which they have shown to be robust to nonequilibrium gas physics with cosmological simulations. Though suited to understanding the physical processes that add statistical scatter to $\mathrm{SZ}-M$ or similar relations, these samples are too small to assess the effect of the scatter on the determination of cosmological parameters, especially the effect of relatively rare, major merger events on the mass estimate of similarly rare massive clusters. To include these rare events and focus expressly on the role of mergers on SZ$M$ relations and cosmological parameter estimates, we take a semi-analytic approach that avoids simulating every possible merger within a cosmological framework.

Specifically, we carefully examine the evolution of the SZ observables $Y$ and $y_{\max }$ for a discrete set of detailed N-body/hydrodynamical simulations of binary cluster mergers, generalize the results by identifying and parameterizing the major transient features, or boosts, and then apply these boosts to the merger histories of many clusters generated semi-analytically via computationally cheaper merger trees. We closely follow the methodology of Randall et al. (2002, hereafter RSR), who similarly investigated the effect of merger boosts on the X-ray observable mass proxies $L_{X}$ and $T_{X}$, the X-ray luminosity and temperature respectively, and the bias such boosts induce upon estimates of $\Omega_{M}$ and $\sigma_{8}$ from the inferred MFs.

To assess the impact of a particular world model or cosmology on our results, we consider a "flat" cosmology with a cosmological constant, i.e. the $\Lambda$ CDM concordance model, along with an "open" and Einstein-de Sitter ("EdS") world model for comparison; the relevant parameters are summarized in Table 2.1. The dark energy equation of state and its evolution are only examined for the flat universe. The Hubble constant is parameterized as $100 \mathrm{~h} \mathrm{~km} / \mathrm{s} / \mathrm{Mpc}$ throughout. 
In this paper, we assess the transient boosting of the SZ observables $Y$ and $y_{\max }$ during cluster mergers, and the systematic influence of mergers on cosmological parameter values derived from inferred cluster MFs. In $\S 2.2$ we describe the binary cluster merger simulations from RS and the evolution of $Y$ and $y_{\max }$ during mergers. In $\S 2.3$ we discuss the generation of cluster merger histories from merger trees created via the extended Press-Schechter formalism (Press \& Schechter 1974; Lacey \& Cole 1993), fit analytic functions that describe the transient behavior of merger boosts in the simulations, and generalize these functions to the entire family of possible mergers. In $\S 2.4$ the effect of boosts on the $\mathrm{SZ}-M$ relations are analyzed, in $\S 2.5$ the distribution function proxies for the MF and the effect of boosts on them are described, and in $\S 2.6$ the distribution functions are used to assess the impact of mergers on the cosmological parameters $\Omega_{M}, \sigma_{8}$, and $w$. Our results are discussed and summarized in $\S 2.7$.

\subsection{Merger Simulations}

To infer the effect of mergers on the SZ properties of clusters, detailed N-body/hydrodynamical simulations of every conceivable combination of cluster mass and impact parameter would be ideal. A realistic alternative is to use a small but representative set of sim-

Table 2.1. Cosmological Parameter Values Used to Create Merger Trees

\begin{tabular}{lccc}
\hline \hline Model & $\Omega_{M}$ & $\Omega_{\Lambda}$ & $\sigma_{8}$ \\
\hline Flat & 0.3 & 0.7 & 0.834 \\
Open & 0.3 & 0.0 & 0.827 \\
EdS & 1.0 & 0.0 & 0.514 \\
\hline
\end{tabular}


ulated mergers $(\mathrm{RS})$ and interpolate or extrapolate from them the expected behavior of SZ observables for any set of merger parameters.

A detailed description of the simulations can be found in RS. Eight simulated binary cluster mergers were available with 3 mass ratios $M_{>} / M_{<}=1,3$, and 6.5 each for 3 impact parameters $b=(0,2,5) r_{s}$ except the $M_{>} / M_{<}=6.5, b=2 r_{s}$ case. Here $r_{s}$ is the scale radius in the NFW profile for the more massive cluster (Navarro et al. 1997). In all simulations, the less massive cluster's mass was fixed at $M_{<}=2 \times 10^{14} M_{\odot}$. Note that the $M_{>} / M_{<}=6.5$ simulation runs are not specifically mentioned in RS, although they were generated by the same means as the other simulations.

\subsubsection{Equilibrium $Y-M$ and $y_{\max }-M$ Relations}

To compare the SZ properties of merging clusters with those of similar clusters that are not undergoing mergers, we need an equilibrium $\mathrm{SZ}-M$ relation. The theoretical models of clusters used in RS are designed to represent observed, non-cooling flow clusters and to have X-ray temperatures typical of present day "rich" clusters. These initial conditions therefore include "preheating" and radiative cooling, though radiative cooling is ignored as a dynamic process as the cooling timescale is designed to exceed a Hubble time. Though cooling is absent in the RS mergers, our results for $y_{\max }$ generally agree with a similar set of cluster simulations (Poole et al. 2007) that do include radiative cooling. In any case, we are interested in the change of $Y$ or $y_{\max }$ due to mergers and not in precisely characterizing the equilibrium state of clusters. To accurately assess the relative effect of mergers on the SZ effect, we take the initial clusters in RS as our equilibrium clusters, which should correspond well to actual clusters since they were built to resemble observed, relaxed clusters. 
Self-similar scaling relations derived from virial arguments (da Silva et al. 2004; Cohn \& Kadota 2005) give $Y \propto M^{5 / 3} f_{g}$, where $M$ is the virial mass and $f_{g}$ is the gas mass fraction. For masses $M \gtrsim 10^{14} M_{\odot}, f_{g} \propto M^{1 / 3}$ though $f_{g}$ steepens at smaller masses. This general trend of increasing $f_{g}$ with mass has been observed for relaxed, nearby clusters (Vikhlinin et al. 2006). Assuming all clusters have similar density profiles, we find that $y_{\max } \propto M f_{g}$. For the initial clusters in the simulations, we calculate exact solutions for $Y$ and $y_{\max }$ :

$$
\begin{gathered}
Y=0.210\left(\frac{M}{10^{15} M_{\odot}}\right)^{2}\left(\frac{r_{s}}{\mathrm{kpc}}\right)^{-1} f_{g} h^{-2} \mathrm{Mpc}^{2}, \\
y_{\max }=8.84 \times 10^{3}\left(\frac{M}{10^{15} M_{\odot}}\right)^{2}\left(\frac{r_{s}}{\mathrm{kpc}}\right)^{-3} f_{g} .
\end{gathered}
$$

Here, $r_{s}$ and $f_{g}$ are found numerically (equations (20)-(23), RS). Over the range of cluster masses we consider, $Y$ and $y_{\max }$ scale approximately as $Y \propto M^{2}$ and $y_{\max } \propto M^{1.3}$.

In practice, we fit the numerical solutions for $Y(M)$ and $y_{\max }(M)$ each to a power law times a 13 degree polynomial. The high order of the polynomial is required primarily because we need the derivatives of the function to compute the $Y$ and $y_{\max }$ distribution functions ( $\mathrm{YF}$ and $\mathrm{yF}$ respectively). The fractional error in the derivatives of the fits is $\lesssim 1 \%$ for both $Y(M)$ and $y_{\max }(M)$, and better than that for the fits themselves. 


\subsubsection{Merger Boosts to $Y$ and $y_{\max }$}

\section{Generating $Y$ and $y_{\max }$ from the Simulations}

For each simulation in RS, the behavior of the X-ray temperature and luminosity was calculated (see RS, Figures $5 \& 8$ ) as a function of time. We would like similar curves for $Y$ and $y_{\max }$; however, these quantities were not calculated during the simulations, so we need to evaluate them from saved 3D "snapshots" of the simulation grid in order to recreate the evolution with time. For most of the runs, 40 to 60 snapshots were saved fairly regularly over the 14 Gyr the mergers were followed. From the gas pressure distribution, the Comptonization parameters can be calculated individually for each snapshot and combined to trace the evolution of $Y$ and $y_{\max }$ during the merger.

Simulated SZ images for any orientation can be generated for each snapshot. As an example, Figure 2.1 shows $100 \times 100$ pixel images from 2 snapshots of the $M_{>} / M_{<}=3$, $b=2 r_{s}$ merger. For both of these images, our line-of-sight is oriented at $45^{\circ}$ to the merger axis and rotated $45^{\circ}$ azimuthally from the merger plane. In this particular example, the clusters are seen just before and just after the first core crossing, which generally corresponds to the maximum transient enhancement of both $Y$ and $y_{\max }$. Note that while the images look qualitatively similar, the scale of the image after core passage is twice that of the pre-core passage snapshot, suggesting that both $Y$ and $y_{\max }$ should get "boosted" during a merger event.

To compute $Y=\int y d A=\frac{\sigma_{T}}{m_{e} c^{2}} \int P_{e} d V$, we simply add up the pressure in each computational cell weighted by the cell volume so that $Y=\frac{\sigma_{T}}{m_{e} c^{2}} \sum_{i} P_{e, i} \Delta V_{i}$, where the sum is over all the cells in the $3 \mathrm{D}$ grid. We do not restrict the integration to the virial radius $r_{200}$ or $r_{500}$ as in other cases where $Y$ has been modeled (da Silva et al. 2004; Motl et al. 2005) for several reasons. First, the initial conditions for 

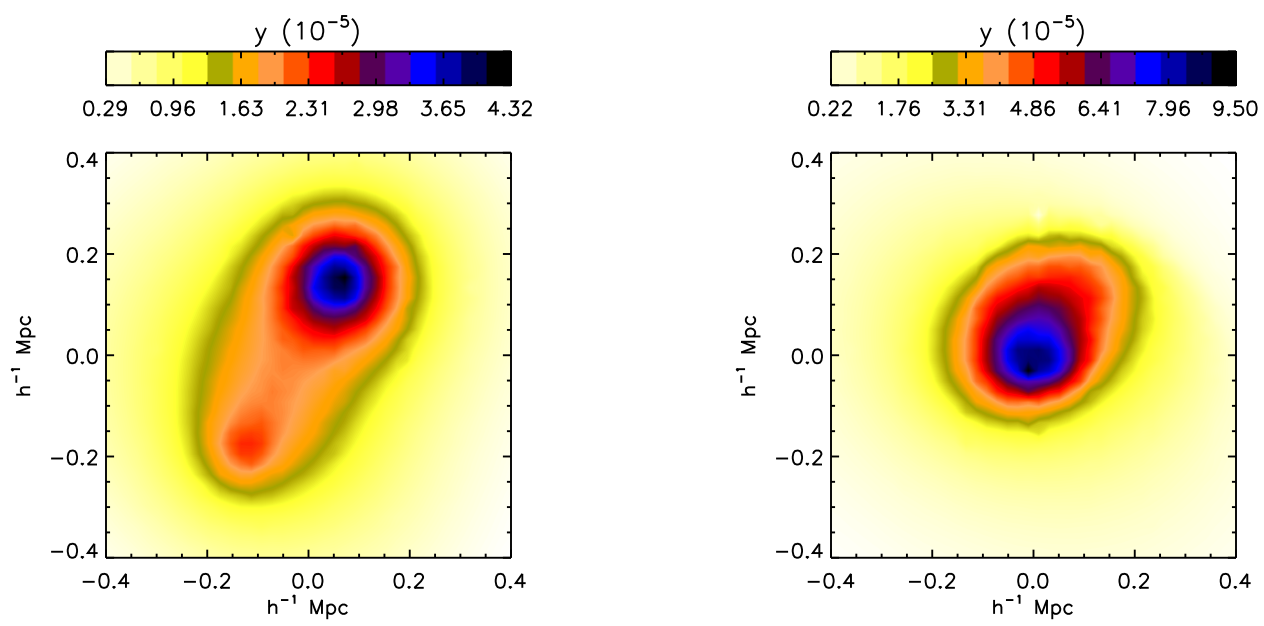

Fig. 2.1. - Images of the SZ parameter $y$ from 3D snapshots of the 1:3 mass ratio, $2 r_{s}$ impact parameter merger simulation. Here $r_{s}$ is the NFW scale radius of the more massive cluster. Both images are viewed from a line-of-sight which is rotated $45^{\circ}$ from the merger axis and $45^{\circ}$ azimuthally from the merger plane. Left: $386 \mathrm{Myr}$ before first core crossing. Right: 114 Myr after first core crossing.

the simulated clusters cut off the pressure and density profile at the virial radius, so these definitions are at least initially equivalent. Also, during the merger there is no such well-defined radius as the gas is interacting violently. However, nearly all of the contribution to $Y$ comes from gas inside the virial radius: $99.5 \%$ initially and $95 \%$ after the clusters have merged and equilibrated.

For each snapshot of each merger simulation, values of $y_{\max }$ are computed for 339 orientations of the merger relative to our line of sight. Because the effects of the merger on the value of $y_{\max }$ tend to vary the most near the merger axis, we more finely sampled the viewing angles in this direction. The orientations sampled with respect to the merger axis are uniformly spaced in $\sin \theta$, where $\theta$ is the polar angle, such that $\Delta \sin \theta=1 / 15$. The sampling of the azimuthal angle $\phi$ is varied, to ensure relatively even spacing, as $\Delta \phi=8^{\circ} / \sin \theta$. To determine $y_{\max }$ for each merger, snapshot, and orientation, values of $y$ were computed by integrating along 16 lines 
of sight (equation (2.1)), on a $4 \mathrm{x} 4$ grid, to form an SZ image of the cluster as seen from that orientation. The grid was then recentered on the maximum value of $y$ and reduced in scale by a factor of 3.5, and $y$ was calculated again. This procedure was repeated until the maximum value on the grid varied by less than $0.1 \%$ compared to the value from the previous iteration, and this $y$ is adopted as $y_{\max }$.

\section{Correcting for Mass Loss Outside the Grid}

During each merger, some gas is flung out to large radii and lost from the simulation due to the finite size of the computational grid and outflow boundary conditions at the grid edge. Of course, once the gas is outside the simulation grid, it is permanently lost. Noticeable amounts of gas do not leave the grid until after the first core passage. Since we are mainly interested in the times when the merger boost is large, which occurs near the peak associated with first core passage, our results are not particularly affected by the lost gas. At late times, however, after the clusters have merged, $Y$ remains below the expected value for a cluster with mass $M_{\text {total }}=M_{<}+M_{>}$. Since at these cluster masses $Y \propto M^{2}$ and $y_{\max } \propto M^{1.3}$, we correct for the lost gas by taking

$$
Y=\left[\frac{M_{\text {gas }}(t=0)}{M_{\text {gas }}(t)}\right]^{2} Y_{\text {calc }}
$$

and

$$
y_{\text {max }}=\left[\frac{M_{\text {gas }}(t=0)}{M_{\text {gas }}(t)}\right]^{1.3} y_{\text {calc }} .
$$

Here, $Y_{\text {calc }}$ and $y_{\text {calc }}$ are the integrated and maximum SZ parameters calculated by integration over the grid prior to this correction. The correction is small; over the duration of the first peak in $Y$, which is much longer than the peak in $y_{\max }$, less than $5 \%$ of the gas has been lost from the grid. 
In fact, some of the lost gas exits the grid near to or above escape velocity, assuming a collisionless ballistic trajectory, so correcting for its loss may seem inappropriate. The majority of the gas, except during the short period after the first core passage, effectively leaks out of the grid due to a lack of pressure support at the simulation boundary. This artificially lower pressure propagates inward, requiring the correction we apply; otherwise, the boost will be slightly underestimated. After clusters have formed in cosmological numerical simulations, the gas fraction at the virial radius is generally 10\% below the cosmic baryon fraction (Crain et al. 2007; Eke et al. 1998), perhaps indicating that up to $\sim 5 \%$ of the gas has been ejected, given that $5 \%$ of the baryons are in stars. The simulations of RS we utilize cannot accurately follow the merger to its true final state and so we cannot address the question of true gas ejection from clusters after merger events. However, the initial simulated clusters are constructed to match observed clusters with realistic gas fractions, so if gas is in fact lost, that effect is intrinsically included by $\mathrm{RS}$ and the resultant boosts in $Y$ and $y_{\max }$. Our conclusions are not drawn from any late time evolution in the simulations, nor do we investigate the true post-merger state of clusters.

Additionally, we correct $Y$ for the slight evolution at large radii in the relaxed, pre-merger profiles of the simulated clusters. Because the integrated Comptonization parameter is inversely proportional to a low power of cluster radius due to $Y \propto n_{e}$, the outer parts of a cluster contribute significantly to its overall value, as compared to $L_{X}$, which is proportional to $n_{e}^{2}$. The lower pressure in the outer regions can affect $Y$ because there is more volume at large radii, even though $y_{\max }$ remains unaffected. We observe a slight drop in the pressure profile outside the central core over time before the individual clusters begin to interact, which is likely due to the artificial truncation of gas at the virial radius - gas at this boundary is not in hydrostatic equilibrium 
in the simulations and will flow outward, and the loss of pressure support will travel inward, readjusting the profile as the system tries to establish hydrostatic balance. While for the least massive cluster this effect is hardly noticeable, the magnitude of the effect increases with total cluster mass. Fortunately, the effect on $Y(t)$ appears to be linear in time, so we correct the time evolution of $Y$ such that $Y$ is forced to be constant before the clusters begin to interact, normalized to $Y(t=0)$.

\subsubsection{Evolution of $y_{\max }$ and $Y$ During Mergers}

In Figure 2.2, $Y$ and $y_{\max }$ are shown as a function of time for the merger simulations including the corrections described in $\S 2.2 .2$. For $y_{\max }$, the plot is shown for a viewing orientation at $90^{\circ}$ to the merger axis and in the merger plane. The maximum boost for the head-on collision in $y_{\max }$ is nearly a factor of 10, while the boost in $Y$ is always less than a factor of 2 , though the duration of the boost in $Y$ is much longer than that for $y_{\max }$. Motl et al. (2005) report a maximum boost factor in $y_{\max }$ of 20 in cosmological simulations re-simulated to include gas hydrodynamics, twice the amount of boosting we find, though their result could be due to an artificially high central temperature in their pre-merger clusters (Loken et al. 2002). However, it is more likely the enhanced boost is due to the natural inclusion of multiple mergers and constant accretion along filaments, which are not included in binary merger simulations. For example, a triple merger between 2 equal mass clusters and a third subcluster with a tenth of one of their masses should yield a boost factor of 20, extrapolating our results to such a case. Additional pressure due to bulk motions within the pre-merger clusters, producing stronger shocks, may also lead to a larger boost. Globally, the temperature profile of the initial clusters in RS agrees well with those clusters assembled in cosmological simulations (Loken et al. 2002), so the precise origin of the discrepency is unclear. 
However, our boost factors are confirmed in a recent set of binary cluster mergers (Poole et al. 2006), in which Poole et al. (2007) find $y_{\max }$ to be boosted by a factor of $\sim 10$ (see their Figure 7 ).

Essentially, $y_{\max }$ traces the densest parts of clusters, which are the cores. These remain reasonably intact until near the time of first core crossing, which makes the peak in $y_{\max }$ relatively narrow. On the other hand, $Y$ involves a sum of all the gas, so it begins to get boosted as soon as gas at large radii starts to interact, long before the cores approach, and the boost lasts longer, as gas in the outer regions needs more time to re-equilibrate. The time evolution of $y_{\max }$ is qualitatively similar to that found by RS for the X-ray temperature and luminosity, quantities that are also dominated by the cores of clusters due to the fact that the X-ray emissivity depends on the square of the density.

The plots of the evolution of $Y$ already indicate that this parameter will not be strongly affected by mergers. First, the boosts in $Y$ are smaller than in $y_{\max }$. Second, the boosts are not large compared to the equilibrium effect of increasing the mass. Assuming $Y \propto M^{2}$, the boost factor $B$ needed to exceed the final equilibrium value of $Y_{\text {final }}$ is $B>\frac{Y_{\text {final }}}{Y_{1}+Y_{2}}=\frac{\left(M_{1}+M_{2}\right)^{2}}{M_{1}^{2}+M_{2}^{2}}$. For equal mass mergers, this condition gives $B>2$, and from Figure 2.2 it is clear the boost factor is always $<2$. If $Y$ is used as a proxy to determine the mass of a cluster, the resulting value during the merger will nearly always lie between the individual initial masses of the subclusters and the final total mass. In a certain sense, this only affects the definition of when the cluster has merged, and the applicable mass, and does not represent a real bias. The other mass ratios considered here can boost $Y$ beyond the final equilibrium value, but only by factors slightly larger than unity. 

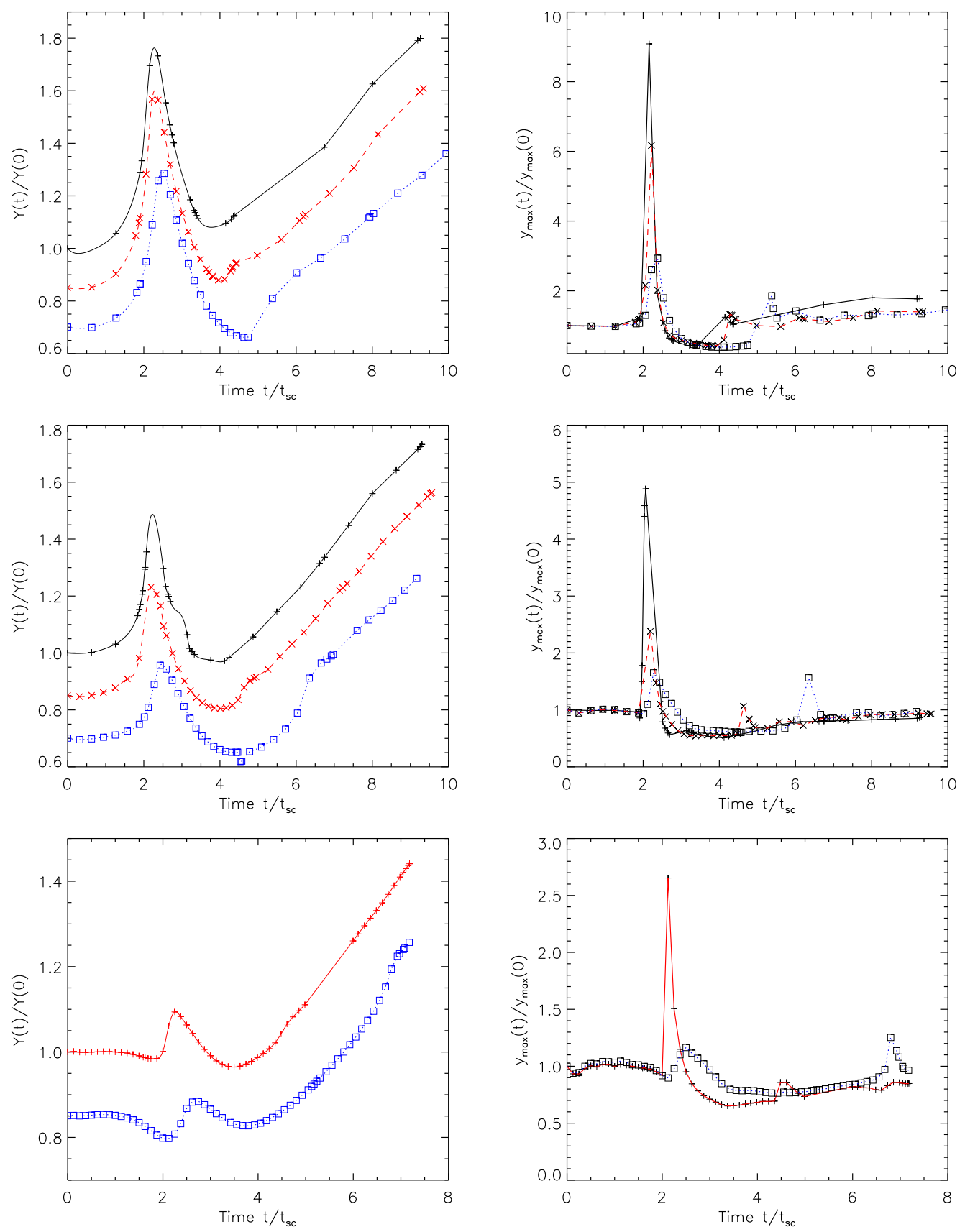

Fig. 2.2. - Evolution of the SZ effect during a merger. In each panel, different curves are for different values of the merger impact parameter: $b=0$ (solid, black line), 2 $r_{s}$ (dashed,red line), and $5 r_{s}$ (dotted, blue line), where $r_{s}$ is the NFW scale radius of the more massive cluster. In the left panels, the $b=2 r_{s}$ simulation run is offset downward by 0.15 and the $5 r_{s}$ run is offset downward by 0.3 for clarity. The time is scaled by the sound crossing time $t_{\mathrm{sc}}$ of the more massive premerger cluster. Left: Integrated Comptonization parameter $Y$ versus time for the 1:1 (top), 1:3 (middle), and 1:6.5 (bottom) mass ratios. Right: Maximum Comptonization parameter $y_{\max }$ versus time for the 1:1 (top), 1:3 (middle), and 1:6.5 (bottom) mass ratios. The mergers are observed $90^{\circ}$ to the merger axis and in the merger plane. 


\subsection{Merger Trees}

Structure formation and evolution are most easily traced through the mass function (MF) of dark matter halos, $n(M, z)$, where $n(M, z) d M$ gives the number of halos per unit comoving volume with masses in the range $M \rightarrow M+d M$. Currently, the MF for a given cosmology at a given redshift can be found most accurately from numerical N-body simulations (Springel et al. 2005). While accessing the results of these simulations has become more feasible (e.g., Lemson \& Springel 2006), a semianalytic approach to obtaining the MF proves more practical, especially since we are concerned with the relative effect of merger boosts on the underlying MF and not the precise nature of the MF itself. We follow the PS formalism, which agrees with the MF found in numerical simulations, especially at higher masses (Bryan \& Norman 1998); specifically, we use extended Press-Schechter theory as developed in Bond et al. (1991) and Lacey \& Cole (1993) and applied in RSR. Though the PS formalism fails to reproduce the MF found in numerical simulations at very high redshifts and low cluster masses (see, e.g., Sheth \& Tormen 1999; Lukić et al. 2007), it is more than sufficient over the redshifts $(z=0 \rightarrow 2)$ and masses $\left(M=10^{14} \rightarrow 10^{16} M_{\odot}\right)$ of interest here.

Press \& Schechter (1974) give the MF at some redshift $z$ as

$$
n_{P S}(M, z) d M=\sqrt{\frac{2}{\pi}} \frac{\bar{\rho}}{M} \frac{\delta_{c}(z)}{\sigma^{2}(M)}\left|\frac{d \sigma(M)}{d M}\right| \exp \left[-\frac{\delta_{c}^{2}(z)}{2 \sigma^{2}(M)}\right] d M
$$

where $\sigma(M)$ is the current rms density fluctuation within a sphere of mean mass $M$, and $\delta_{c}(z)$ is the critical linear overdensity required for a region to collapse at redshift z. The derivation of this expression assumes that halos grow from Gaussian density fluctuations that have larger amplitudes on smaller scales. Structure then forms 
hierarchically, with small halos collapsing first and merging to form larger halos. In this scenario, the highest mass halos, observed as clusters of galaxies, form most recently and should be most affected by merger processes at the present day.

From this extended PS formalism, we follow the procedure outlined in $\S 3$ of RSR, in which a "merger tree" is generated for a present day cluster. The merger tree traces the merger and accretion history of a cluster of mass $M$ back in time. For each time step, a progenitor cluster of mass $M_{p 1}$ is chosen from a probability distribution (Lacey \& Cole 1993, equation (2.25)), and since we only consider binary mergers, the mass of the other progenitor cluster is given by $M_{p 2}=M-M_{p 1}$. We will use the notation $M_{>}$and $M_{<}$for the larger and smaller masses of the subclusters in each binary merger. RSR in $\S 3.1$ discusses the disadvantages of dealing solely with binary mergers; however, our set of simulated mergers does not address more complex mergers, so we have no good way to derive a boost for them. Also, boosts are most dramatic for near equal mass mergers, and in such cases additional merger participants will likely be much less massive and have a negligible effect on the resultant boosts. However, one result of ignoring multiple mergers is that the merger tree-derived MF tends to overestimate the analytic PS MF for $z>0$; while we start with the correct MF at $z=0$, it will become more and more biased with redshift due to this omission. The progenitor cluster with mass $M_{p 2}$ is not taken from the PS distribution and is generally overestimated, so that the high mass end of the MF is overestimated at the expense of the very low mass end. Since we concern ourselves with the highest mass clusters, our resulting MFs will lie slightly above the analytic prediction, as illustrated in Figure 2.6, which we must take into account when fitting MFs in $\S 2.6$.

A large number of merger trees was created with a broad span of initial cluster masses, and the distribution of the initial masses was weighted so as to give the present 
day mass function. From ensembles of merger trees for the cosmologies of interest, we can find the MF at any redshift, and at any redshift we have each cluster's merger history, which can be used to determine the merger boost in some observable - in our case $Y$ and $y_{\max }$.

Merger trees are a simple and computationally cheap way to simulate structure formation for a particular world model. But they are limited in that they only specify progenitor cluster masses and discrete time intervals during which the mergers occur. All the dynamics and other details of a merger, however, are absent from EPS-derived merger trees. The information needed to connect the trees to our merger simulations is the masses of the clusters, the impact parameter $b$ of the encounter, and the time of first core passage, which we designate as the time of the merger, $t_{\text {merge }}$, in the merger trees. While the masses are provided by the merger trees, an appropriate $b$ must be selected for each merger in the trees. We follow the method in $\S 6$ of RSR, where a value for the spin parameter (see their text for its definition) is chosen from a Maxwell-Boltzmann-like distribution, which represents the observed distribution from numerical simulations (Bullock et al. 2001), allowing $b$ to be derived from the chosen spin parameter (Sarazin 2002). To determine the precise value of $t_{\text {merge }}$, we simply select a random time within the small discrete time step used in the merger trees, and take that time to be the instant of first core passage, since the merger could have occurred at any point within that time.

\subsubsection{Merger Boost Histograms}

As discussed in RSR, the effect of a merger boost on a cluster whose history is characterized by a merger tree can be determined from a histogram which gives the magnitude of the boost as a function of time. Since the merger trees give a 
statistical description of the history of cluster mergers, it is sufficient to determine the distribution histogram of boosts versus the observed time $t_{\mathrm{obs}}$. The form of the histogram reduces the details contained in the curves in Figure 2.2 to a simpler, oneto-one function that can be fit by the merger parameters $M_{<}, M_{>}$, and $b$. In the fits, we scale the impact parameter $b$ by the core radii of the two merging clusters, $b^{\prime}=b /\left(r_{c<}+r_{c>}\right)$ and the time by the ratio of the virial radius of the more massive cluster to the gas sound speed, $t_{\mathrm{sc}}$. A more detailed explanation of these scalings is given in $\S 5.3$ of RSR.

\section{Fitting $Y$ Histograms from Simulations}

The left panel of Figure 2.3 shows the cumulative time spent by the system above any given value of $Y$ for the $M_{>} / M_{<}=1$ merger simulations. We use cubic spline interpolation of the boost curves in the left panel of Figure 2.2 to produce a smoothly varying histogram. In RSR, the $T_{X}$ and $L_{X}$ boost histograms were well-fit by hyperbolas parameterized by the equations given in their Appendix B. We find hyperbolas also well-describe the $Y$ histograms, and we use the same parameterization as RSR with only a minor change given in Appendix A.

\section{Fitting $y_{\max }$ Histograms from Simulations}

The procedure for $y_{\max }$ is slightly more complicated due to the orientation-dependence of the central Comptonization parameter. The evolution of the maximum value of $y$ as a function of viewing angle varies more dramatically near the merger axis than perpendicular to it. As noted in $\S 2.2 .2$, for each merger simulation, the evolution of $y_{\max }$ with time was calculated for 339 orientations, sampling more finely around the merger axis. Because $y_{\max }$ really traces the cluster cores, the peaks in the curves are 

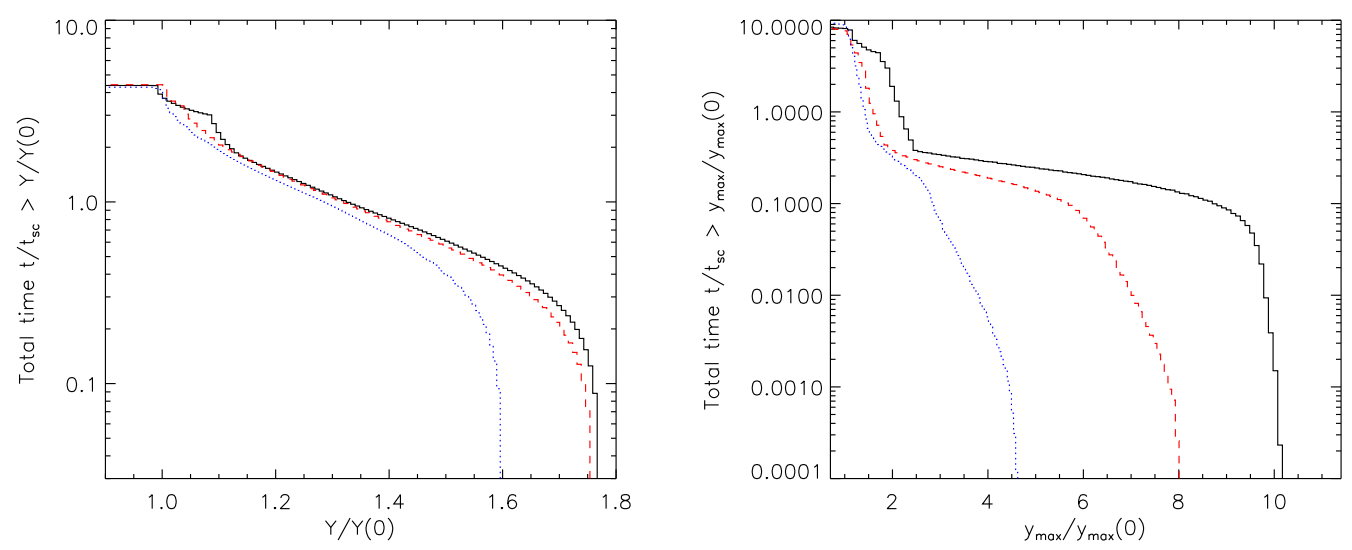

Fig. 2.3. - Left: Histogram of the total time the integrated Comptonization parameter $Y$ is above some fraction of its initial premerger value $Y(0)$, scaled by the sound crossing time $t_{\mathrm{sc}}$ of the more massive premerger cluster. Histograms are shown for equal-mass mergers at 3 impact parameters $b=0$ (solid, black line), $2 r_{s}$ (dashed, red line), $5 r_{s}$ (dotted, blue line), where $r_{s}$ is the NFW scale radius of the more massive cluster. Right: Histogram of times for $y_{\max }$.

larger and have shorter durations. As a result, we found that simple interpolation did not sufficiently sample the peaks, so we use a superposition of Gaussians to fit the shape of the boost as a function of time.

The merger trees contain no information about the orientation of the cluster mergers, and we assume an isotropic distribution relative to our line-of-sight. For our grid of 339 viewing angles, the probability of any one orientation is determined by the solid angle of that grid cell. We weight each orientation by this solid angle divided by $4 \pi$. All $339 y_{\max }(t)$ curves, weighted by their probability of being observed, are used to construct a histogram like those described in $\S 2.3 .1$. The histograms for the $M_{>} / M_{<}=1$ runs are shown in the right panel of Figure 2.3. Since these histograms include the distribution of merger boosts for all orientations of the line of sight, the boosts need to be normalized to the pre-boost value of $y_{\max }$ for some fixed orientation. The boosts in the right panel of Figure 2.3 were taken relative to the pre-boost $y_{\max }$ 
as observed $90^{\circ}$ to the merger axis and in the merger plane. Note that this is the same orientation assumed in the right panels of Figure 2.2.

Because the $y_{\max }$ histograms include the results from many different orientations, the high boost ends of the histograms decline more slowly with time than for $Y$ or $L_{X}$ or $T_{X}$. A different function is thus used to fit these histograms (see Appendix A).

\subsubsection{Generalizing Merger Boosts for Arbitrary Mass Ratio and Impact Parameter}

As in RSR, the parameters of the fits to the boost histograms were fit to simple functions of the masses and impact parameter in the merger. The forms of these functions were chosen so as to have the correct asymptotic forms (e.g., in the limit of large $\left.M_{>} / M_{<}\right)$. The free parameters of these functions were chosen to best fit the histograms from all 8 simulation runs. The values of these parameters are given in Table A.1 (below in the Appendix) for the $Y$ and $y_{\max }$ histograms.

The maximum fractional error in the fits to the boost simulation data for $Y$ is $<3 \%$ except for the 2 runs with $M_{>} / M_{<}=6.5$. Here, the evolution of the pressure distribution in the more massive cluster before collision dominates the time evolution of $Y$ (see $\S 2.2 .2)$. The fits overestimate the boosts for the $M_{>} / M_{<}=6.5$ simulations; however, the boosts themselves are small in this case, and the errors are still $<10 \%$.

For the $y_{\max }$ fits, the average fractional error is typically $4 \%$, and the maximum error is $<10 \%$. We found that the time sampling for the $M_{>} / M_{<}=3, b=2 r_{s}$ simulation run was too sparse around the boost to strongly constrain the shape of the $y_{\max }$ histogram, so we did not use this run in our fits. 


\subsubsection{Adding Boosts to Merger Trees}

With the fitted forms for the histograms for the strength of a boost versus time as a function of the masses of the merging subclusters $M_{<}$and $M_{>}$and the impact parameter $b$, the boosted values of $Y$ or $y_{\max }$ can easily be found for clusters from their past merger histories given by the merger trees. For any redshift or observed time, $t_{\text {obs }}$, we search back through a cluster's merger tree and for every merger event, we find the boosted value of $Y$ or $y_{\max }$ for that merger. If the boosted $Y$ or $y_{\max }$ exceeds the value given by our equilibrium equations (2.3) and (2.4) for the mass of the cluster at $t_{\text {obs }}$, then we assign the boosted value to that cluster's observed $Y$ or $y_{\max }$; otherwise it acquires its equilibrium value. Boosted values less than those given by the equilibrium equations are not allowed because the analytic fits from which boost factors are derived poorly describe the histograms, such as those shown in Figure 2.3, for negative and small positive boosts. While the discrepency between the simulation-based histograms and the analytic fits for small boosts leads to an underestimate of the number of these clusters, we are primarily concerned with the more dramatic effects caused by large boosts, which are well-described by the fits.

\subsection{SZ versus Mass Correlation}

Once clusters observed at some redshift are assigned values of $Y$ and $y_{\max }$ based on each cluster's merger history, we can evaluate the robustness of the $Y-M$ and $y_{\max }{ }^{-}$ $M$ relations. The top panels of Figures 2.4 and 2.5 show $Y$ and $y_{\max }$ versus mass for clusters in our merger trees at $z=0$ and $z=1$. Most clusters have nearly unboosted values of $Y$ and $y_{\max }$, while the number of clusters that deviate from either $\mathrm{SZ}-M$ relation drops roughly exponentially with the strength of the boost. We find that 


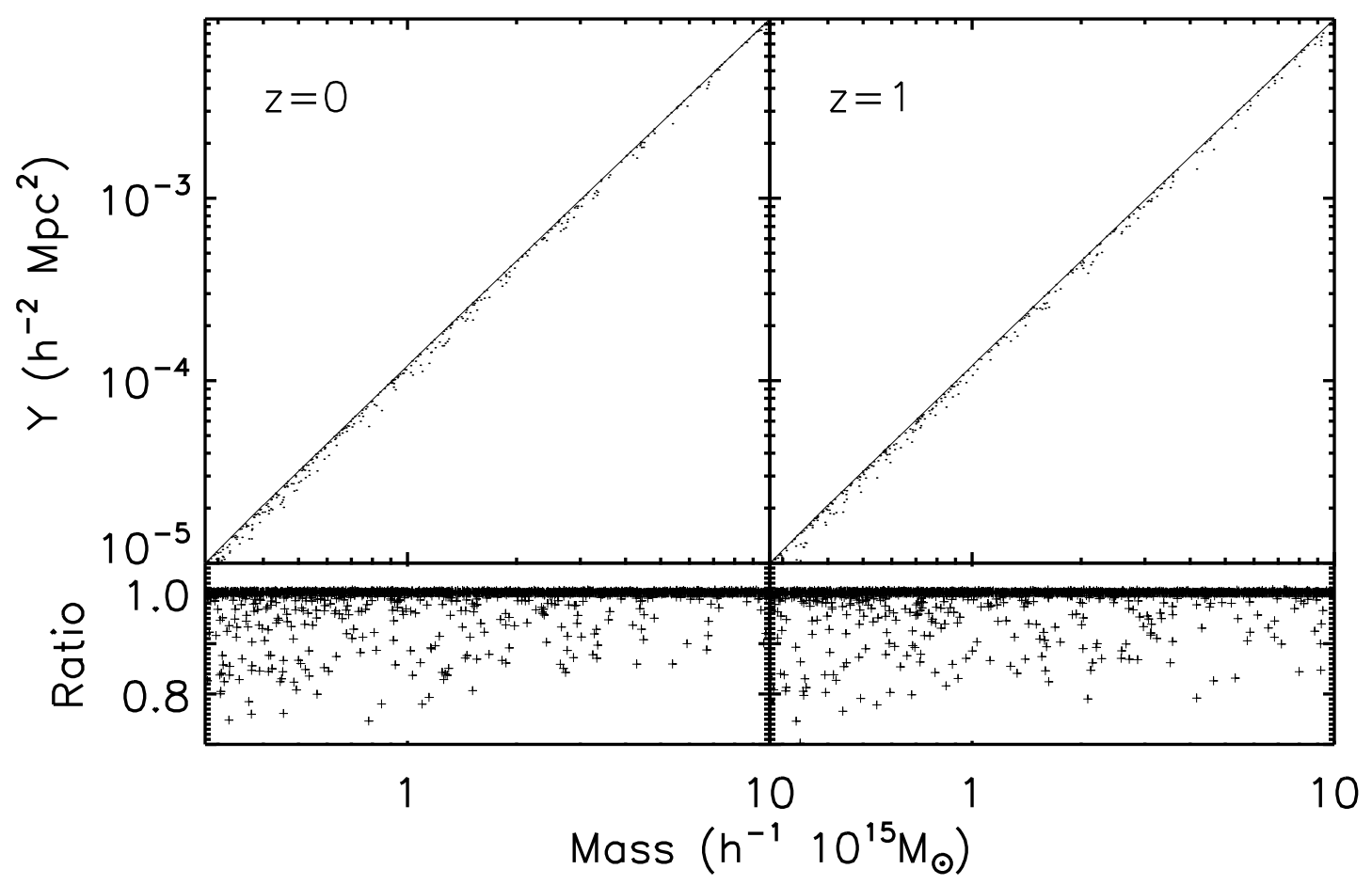

Fig. 2.4. - Integrated Comptonization parameter $Y$ (top panels) versus total mass in the flat cosmology at $z=0$ (left panels) and $z=1$ (right panels) for clusters with $Y>10^{-5} h^{-2} \mathrm{Mpc}^{2}$. The combined mass of both merging clusters is used if $Y$ is boosted or $t_{\mathrm{obs}}>t_{\text {merge }}$, where $t_{\text {merge }}$ is the time of maximum boost. The apparent solid line is the result of many individual clusters at or near their equilibrium values of $Y$. In the bottom panels, the ratio of the boosted clusters to their equilibrium values for each redshift is shown. Each panel contains 5190 clusters.

$\sim 15 \%$ of clusters are boosted in $y_{\max }$ by $\gtrsim 15 \%$ and in $Y$ by $\gtrsim 0.1 \%$. Note that the scatter in Figures 2.4 and 2.5 is due entirely to merger boosts and does not include observational error or scatter related to other physics.

As expected, many clusters are found to have significantly boosted values of $y_{\max }$, which overestimate the actual masses. However, there are almost no "boosts" to $Y$ in Figure 2.4. Instead, we see clusters scattered below the $Y-M$ relation, as is also seen, though to a lesser extent, in Figure 2.5. Clusters that fall below the $Y-M$ relation were "observed" after a merger (after the peak of the boost), but before virialization. 


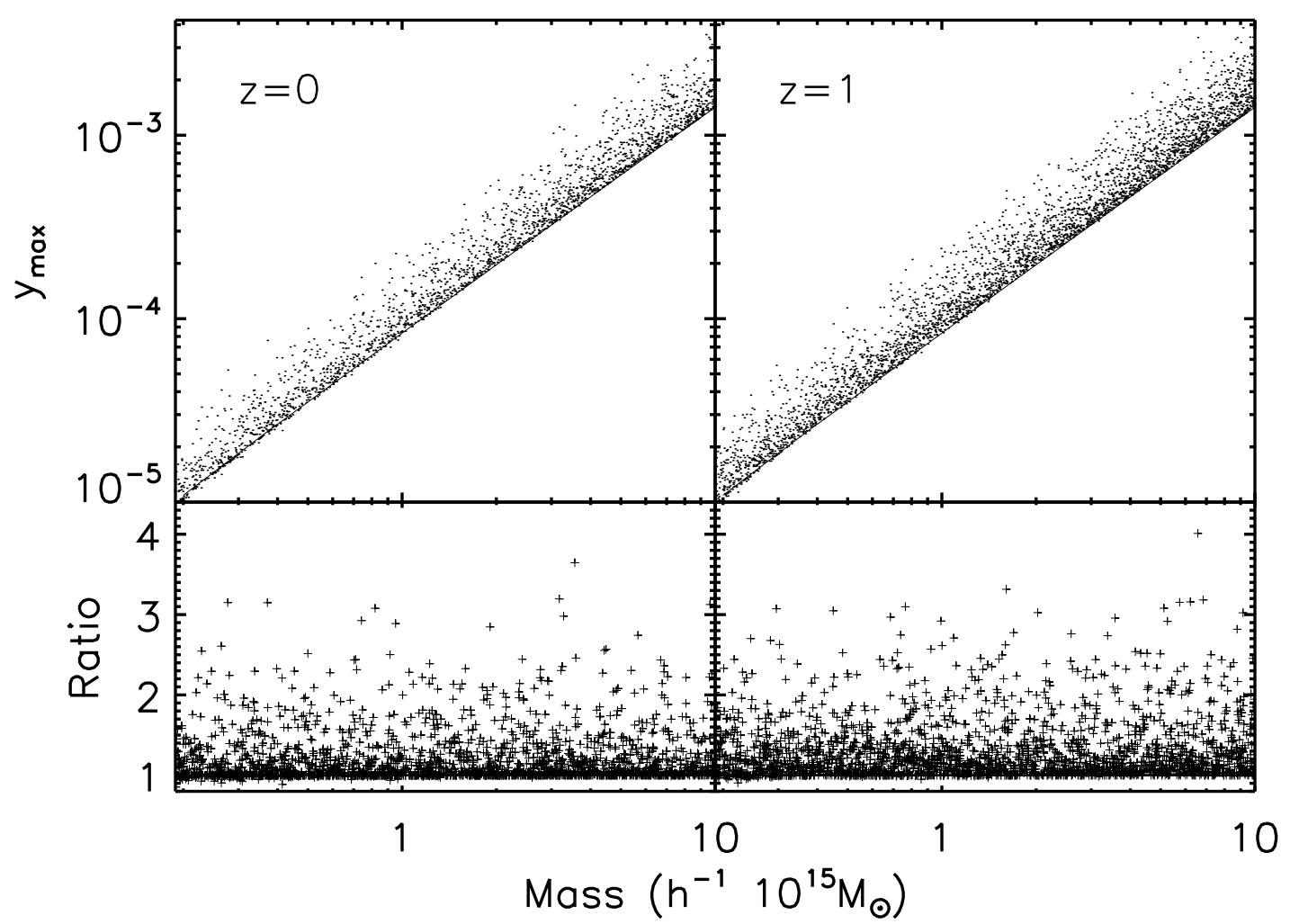

Fig. 2.5.- Same as Figure 2.4, but for the maximum Comptonization parameter $y_{\max }$, for clusters with $y_{\max }>10^{-5}$. Each panel contains 5663 clusters.

It should be noted that, according to $\S 2.3 .3$, a boost is only applied if it gives a $Y$ or $y_{\max }$ greater than its equilibrium value before the merger, while in Figures 2.4 and 2.5 the mass is taken to be the final, or merged, mass of the clusters. So, though clusters can never fall below their pre-merger equilibrium relation in our formulation, a boosted cluster may fall below its post-merger value. The scatter below the $y_{\max }-M$ relation is less pronounced due to the shorter period when the SZ effect is below the eventual equilibrium value (see Figure 2.2). This feature is a general characteristic of observing a recent post-merger cluster and will be difficult to identify as such in an actual survey, and will likely affect the normalization of either SZ- $M$ relation.

In order to quantify the effect of mergers on the SZ versus mass relations, we fit 
power-law functions of the form

$$
Y=A \times 10^{-5} h^{-2}\left(\frac{M}{10^{15} M_{\odot}}\right)^{\alpha} \mathrm{Mpc}^{2}
$$

or

$$
y_{\max }=A \times 10^{-5}\left(\frac{M}{10^{15} M_{\odot}}\right)^{\alpha},
$$

to all of the clusters with $Y>10^{-5} h^{-2} \mathrm{Mpc}^{2}$ or $y_{\max }>10^{-5}$. We estimate the scatter with respect to the best fit, $\sigma_{\text {fit }}$, and also the scatter and offset with respect to the actual equilibrium relations for the SZ effect (equations $(2.3)$ and $(2.4)$ ), $\sigma_{\text {eq. }}$. We define the scatter as

$$
\sigma_{\text {fit }}^{2}=\frac{\sum_{i}\left(y_{i}-y_{\text {fit }, i}\right)^{2} / y_{\text {fit }, i}^{2}}{N-1},
$$

and $\sigma_{\text {eq }}$ is similarly defined, except $N-1$ is replaced by $N$. The coefficients $A$ and $\alpha$ of the fits along with the scatter are given in Table 2.2. The subscripts "b" and "nb" refer to clusters including merger boosts, and not including these boosts (where the SZ properties are given by the equilibrium relations). Note that we consider a logarithmic distribution as in $\S 2.5$ for the cluster masses, so that the fits in Table 2.2, as well as the points in Figures 2.4 and 2.5, do not reflect the actual MF of clusters.

Because the relative strength of boosts is mainly a function of mass ratio and is only weakly dependent on the absolute masses of the merging clusters, clusters are boosted somewhat uniformly in $Y$ and $y_{\max }$ across masses, which tends to change the normalization of the fit, $A$, and only to a lesser extent the slope, $\alpha$. The inclusion of merger boosts, in the case of $y_{\max }$, could either flatten or steepen the slope. The local mass function is flatter at the low-mass end, so low-mass clusters experience more high-mass-ratio mergers overall than high-mass clusters, thus flattening the $y_{\max }{ }^{-}$ $M$ relation. However, when both minor and major mergers are considered, at any 
Table 2.2. Merger-Induced SZ-M Relations and Scatter

\begin{tabular}{|c|c|c|c|c|c|c|c|c|}
\hline & Model & $z$ & $\sigma_{\mathrm{eq}}$ & $\sigma_{\text {fit }}$ & $A_{\mathrm{nb}}$ & $A_{\mathrm{b}}$ & $\alpha_{\mathrm{nb}}$ & $\alpha_{\mathrm{b}}$ \\
\hline \multirow[t]{9}{*}{$Y$} & \multirow[t]{3}{*}{ Flat } & 0.0 & 0.0205 & 0.0218 & \multirow[t]{3}{*}{12.0} & 11.9 & \multirow[t]{3}{*}{1.91} & 1.91 \\
\hline & & 0.5 & 0.0190 & 0.0205 & & 11.9 & & 1.91 \\
\hline & & 1.0 & 0.0197 & 0.0212 & & 11.9 & & 1.91 \\
\hline & \multirow[t]{3}{*}{ Open } & 0.0 & 0.0207 & 0.0220 & \multirow[t]{3}{*}{12.0} & 11.9 & \multirow[t]{3}{*}{1.91} & 1.91 \\
\hline & & 0.5 & 0.0191 & 0.0207 & & 11.9 & & 1.91 \\
\hline & & 1.0 & 0.0203 & 0.0216 & & 11.9 & & 1.91 \\
\hline & \multirow[t]{3}{*}{ EdS } & 0.0 & 0.0214 & 0.0228 & \multirow[t]{3}{*}{12.0} & 11.9 & \multirow[t]{3}{*}{1.91} & 1.91 \\
\hline & & 0.5 & 0.0194 & 0.0209 & & 11.9 & & 1.91 \\
\hline & & 1.0 & 0.0222 & 0.0235 & & 11.9 & & 1.91 \\
\hline \multirow{9}{*}{$y_{\max }$} & \multirow[t]{3}{*}{ Flat } & 0.0 & 0.292 & 0.241 & \multirow[t]{3}{*}{8.25} & 9.01 & \multirow[t]{3}{*}{1.24} & 1.26 \\
\hline & & 0.5 & 0.361 & 0.271 & & 9.39 & & 1.27 \\
\hline & & 1.0 & 0.412 & 0.289 & & 9.70 & & 1.27 \\
\hline & \multirow[t]{3}{*}{ Open } & 0.0 & 0.293 & 0.246 & \multirow[t]{3}{*}{8.25} & 8.96 & \multirow[t]{3}{*}{1.24} & 1.26 \\
\hline & & 0.5 & 0.329 & 0.256 & & 9.20 & & 1.27 \\
\hline & & 1.0 & 0.375 & 0.279 & & 9.46 & & 1.26 \\
\hline & \multirow[t]{3}{*}{ EdS } & 0.0 & 0.414 & 0.290 & \multirow[t]{3}{*}{8.25} & 9.72 & \multirow[t]{3}{*}{1.24} & 1.27 \\
\hline & & 0.5 & 0.485 & 0.301 & & 10.3 & & 1.27 \\
\hline & & 1.0 & 0.531 & 0.299 & & 10.7 & & 1.28 \\
\hline
\end{tabular}


given time the high-mass clusters are undergoing more merger events (see the relative change in yF over time for low- and high-mass clusters in Figure 2.6). Thus, at any given time a higher-mass cluster has a greater probability of finding itself in the midst of a merger of some type. If the mass function is oversampled at the high-mass end, this effect tends to steepen the $y_{\max }-M$ relation. Because our cluster sample has a uniform distribution in log mass, we oversample the high-mass end relative to the low-mass end. Consequently our $y_{\max }-M$ relation does not exhibit the flattening that we would expect if our cluster sample had been drawn from the correct mass function. As Figure 2.5 shows, both high- and low-mass clusters exhibit the same number of large boosts, but the total number of boosted clusters is greater at higher masses. Most of the high-mass clusters with boosts have small boost factors that are difficult to see in the figure.

The boosted normalization for $Y$ is systematically lower than the nonboosted $A$, but by $<1 \%$. In the case of $y_{\max }$, the normalization increases by $\sim 10 \%$. The offsets to $y_{\max }$ are due as much to clusters with small boost factors as to the rarer cases with very large boosts. Note that these clusters tend to be undergoing weaker mergers, which may be hard to detect. Thus, it may be difficult to expunge these clusters from SZ surveys, and the systematic shift in the $y_{\max }$ versus mass relation may bias cluster samples. The merger-induced scatter to the fit, $\sigma_{\text {fit }}$, is $\sim 2 \%$ for the $Y-M$ relation and 25-30\% for the $y_{\max }-M$ relation, and is nearly independent of the cosmological world model and redshift. The scatter relative to the equilibrium relations, $\sigma_{\text {eq }}$, increases with redshift since the merger rate is higher in the past, whereas the addition of boosted clusters adjusts the normalization $A$ to minimize $\sigma_{\text {fit }}$, so the scatter remains about constant between redshifts. Also, because there are fewer clusters that show boosts in $Y$, the scatter $\sigma_{\text {fit }}$ is dominated by deviations of the equilibrium relation 
from a power-law form, which explains why $\sigma_{\text {eq }}$ tends to be slightly smaller than $\sigma_{\text {fit }}$ for $Y$.

\subsection{Distribution Functions of $Y$ and $y_{\max }$}

We computed the distribution functions for $Y$ and $y_{\max }$, which we refer to as the YF and $\mathrm{yF}$, respectively. The distribution function $\mathrm{YF}$ is $n(Y, z)$, where $n(Y, z) d Y$ gives the number of clusters per unit comoving volume at redshift $z$ which have integrated SZ parameters in the range $Y \rightarrow Y+d Y$. The $\mathrm{yF}$ distribution function $n\left(y_{\max }, z\right)$ is defined in an equivalent manner. To build the YF from a merger tree, we find all the clusters that exist at the "observed" redshift and assign a value of $Y$ according to $\S 2.3 .2$ for the non-boosted YF and $\S 2.3 .2$ for the boosted YF. A cluster with integrated Comptonization parameter $Y_{i}$ is then added to a pre-determined bin $\mathrm{YF}_{j}$ such that $Y_{j}^{\text {bin }} \leq Y_{i}<Y_{j+1}^{\text {bin }}$ and appropriately weighted to convert the actual initial distribution of $z=0$ cluster masses used in the merger trees, $d N / d M^{0}$, to the PressSchechter distribution $n_{P S}$ :

$$
\mathrm{YF}_{j}=\mathrm{YF}_{j}+\frac{n_{P S}\left(M^{0}, z=0\right)}{\frac{d N}{d M_{i}^{0}}\left(Y_{j+1}^{\mathrm{bin}}-Y_{j}^{\mathrm{bin}}\right)}
$$

The initial distribution $d N / d M^{0}$ is logarithmically spaced to ensure good statistics at the high mass end, where clusters are rare, and to avoid creating an excessively large number of merger trees.

For the non-boosted case, the YF or yF can be found directly from the equilibrium relations (equations (2.3) \& (2.4)) and

$$
n_{P S}(Y, z) d Y=n_{P S}(M, z) \frac{d M}{d Y} d Y
$$




$$
n_{P S}\left(y_{\max }, z\right) d y_{\max }=n_{P S}(M, z) \frac{d M}{d y_{\max }} d y_{\max }
$$

with $n_{P S}(M, z)$ from equation (2.7). The derivatives are found from fits to the equilibrium relations (

The agreement between the nonboosted merger tree-derived YFs (yFs) and the analytic Press-Schechter YFs (yFs) is shown in Figure 2.6 for the flat world model. Note that the merger trees seem to slightly overestimate the number of lower $Y$ or $y_{\max }$ (i.e. lower mass) clusters at higher redshifts, which is due to a feature of our merger tree procedure discussed in $\S 2.3$ of this work and $\S 3.1$ of RSR.

The nonboosted and boosted YFs and yFs are also compared in Figure 2.6. The boosted YFs are almost identical to the nonboosted YFs. The deviations from the nonboosted YFs are not systematic and are typically of a few percent and only visible in the residual plot. The boosted yFs, however, lie systematically above the nonboosted yFs at all 3 redshifts considered. The fractional deviation increases with both cluster mass and redshift. The increase with cluster mass shows that rare events involving major mergers of moderate mass clusters compete in frequency with the number of rare, very massive clusters with large equilibrium values of $y_{\max }$. The increase in the bias with redshift is apparently due to the higher merger rate in the past. Clearly, clusters with all values of $y_{\max }$ are getting boosted to higher $y_{\max }$ bins in the yFs over our considered range of $y_{\max }$, which includes only the most massive clusters. Such a significant and systematic bias in the yF will affect estimates of cosmological parameters, as discussed below in $\S 2.6$. 

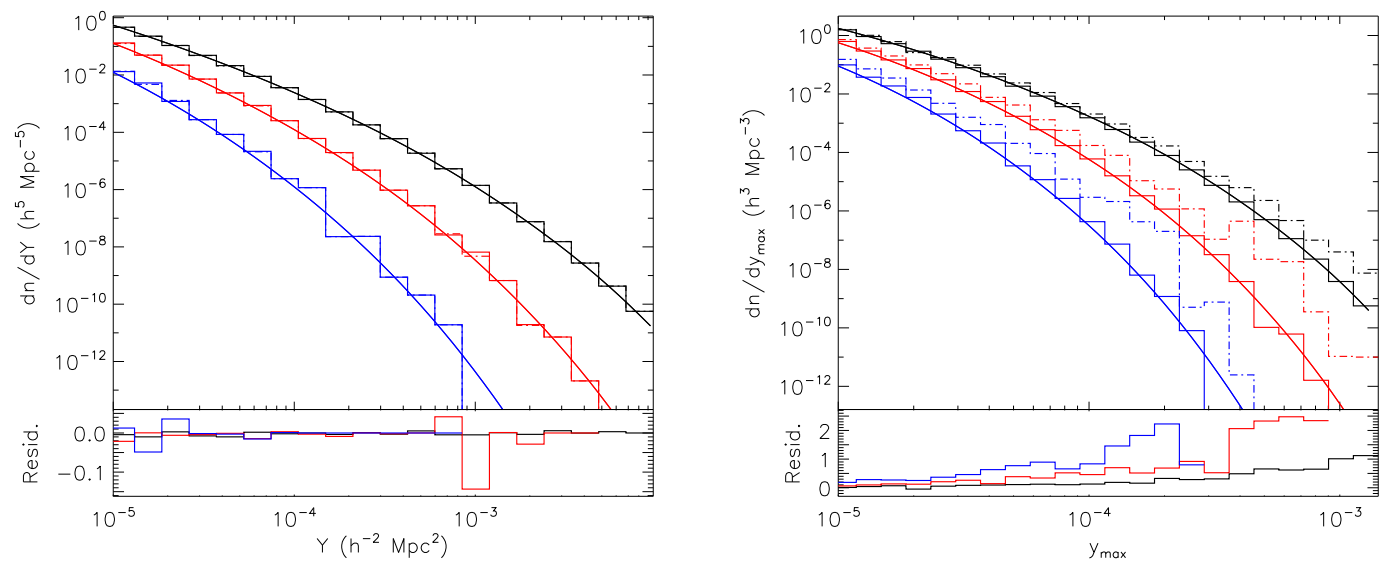

Fig. 2.6.- Boosted (dashed line) versus nonboosted (solid line) integrated Comptonization parameter function YF (left panel) and maximum Comptonization parameter function $\mathrm{yF}$ (right panel) histograms for $z=0$ (top, black), $z=0.5$ (middle, red), and $z=1$ (bottom, blue) in the flat universe. The smooth curves are the analytic PS predictions at each redshift given by equation (2.7). The residual plots give the difference in the logs between the boosted and nonboosted YFs and yFs. Note the significant difference in scales of the residuals between the YFs and yFs.

\subsection{Determining Cosmological Parameters from the Merger Tree YFs and yFs}

Although mergers strongly affect the SZ signals of a small fraction of clusters, because of the exponential high-mass drop-off in the $\mathrm{YF}$ and $\mathrm{yF}$ the effect of mergers on cosmological model fits to these distributions may be significant. To quantify this effect, we derive fits based on the analytic predictions of equations (2.12) and (2.13) to the YF and yF using both boosted and nonboosted merger trees. The differences between the best-fit cosmological parameters derived in the two cases provide an estimate of the systematic bias introduced when merging effects are neglected. 


\subsubsection{Varying only $\Omega_{M}$ and $\sigma_{8}$}

For our 3 cosmological world models, we treat the binned YFs and yFs from the merger trees as observational data and find best-fit values for the parameters $\Omega_{M}$ and $\sigma_{8}$ in equation $(2.12)$ or $(2.13)$. Due to a near degeneracy between $\Omega_{M}$ and $\sigma_{8}$ (Bahcall \& Fan 1998) at a single redshift, we simultaneously fit YFs and yFs for two redshifts: at $z=0$ and at either $z=0.5$ or $z=1.0$. While in practice SZ surveys will observe clusters in a continuous range of redshifts, choosing only 2 redshifts simplifies the fitting procedure and illustrates the effect of merger boosts on these parameters. We choose to only fit clusters above a minimum value of $Y^{\text {min }}=10^{-5} h^{-2} \mathrm{Mpc}^{2}$ or $y_{\max }^{\min }=10^{-5}$. These limits are consistent with the expected detection thresholds for upcoming SZ surveys, such as the AMI, ACT, and SPT projects (e.g., Bartlett 2006), and the likely confusion limit for clusters with $M \lesssim 10^{14} h^{-1} M_{\odot}$ (Holder et al. 2007). These limits also keep our fits from being biased by the large number of clusters at low masses.

To evaluate the extent to which merger boosts affect the estimation of $\Omega_{M}$ and $\sigma_{8}$, we compare their fitted values from the boosted YFs and yFs to the fitted values from the nonboosted YFs and yFs. We do not compare best-fit parameters to the values used to create the merger trees because the trees tend to slightly overestimate the MF, an effect which increases with redshift and is discussed in $\S 2.3$. However, since we are only interested in relative changes to the $\mathrm{YF}$ or $\mathrm{yF}$ due to boosts in $Y$ or $y_{\max }$, this bias in the MF does not affect our results, though the best-fit parameters found from the nonboosted YFs or yFs may differ from the parameter values used to create the trees. Also, any bias caused by our chosen fitting method is accounted for by directly comparing the two YFs or yFs.

The best-fit values of $\Omega_{M}$ and $\sigma_{8}$ for the flat, open, and EdS cosmological world 
models are given in Table 2.3 for both $Y$ and $y_{\max }$. The parameter values used to create the merger trees are summarized in Table 2.1 for reference. In general, the results are independent of world model; cosmological parameter fits tend to be biased in the same direction by about the same amount. However, boosts to $Y$ have almost no effect on fits to $\Omega_{M}$ and $\sigma_{8}$; the changes due to mergers are generally less than $1 \%$ and are not clearly systematic.

In contrast, boosts to $y_{\max }$ significantly bias the values of these parameters: $\Omega_{M}$ is underestimated by $15-30 \%$ and $\sigma_{8}$ is overestimated by $10-20 \%$. The main effect of merger boosts is to increase the number of clusters detected in a particular $\mathrm{yF}_{j}$ bin; in other words, there is a systematic increase in the $\mathrm{yF}$, as shown in the right panel of Figure 2.6. An overall increase in the normalization of the yF leads to an increase in the normalization of the spectrum of initial density perturbations, $\sigma_{8}$. The total matter content, $\Omega_{M}$, is also sensitive to the normalization, but it is nearly degenerate with $\sigma_{8} \approx 0.6 \Omega_{M}^{-1 / 2}$ (Bahcall \& Fan 1998) at a given single redshift. However, $\Omega_{M}$ is more sensitive to the change in the yF over time - the greater the density of matter, the faster structure will grow. If various cosmologies with nearly identical yFs at $z=0$ are considered, those cosmologies with smaller values of $\Omega_{M}$ (and thus larger values of $\sigma_{8}$ ) would produce yFs at $z>0$ that lie above the yFs of cosmologies with larger $\Omega_{M}$ values. As described in $\S 2.5$, merger boosts raise the yF most strongly at higher redshifts, so the change in the $\mathrm{yF}$ from one redshift to another is smaller than for nonboosted yFs, indicating a slower structure growth rate and therefore a smaller $\Omega_{M}$. The overall effect of mergers seems to vary with redshift; $\Omega_{M}$ and $\sigma_{8}$ are found to be less biased when utilizing the $\mathrm{yF}$ at higher redshift $(z=1)$ even though this $\mathrm{yF}$ is fractionally more biased than the yFs at $z=0$ or $z=0.5$. 
Table 2.3. Best-Fit Values for $\Omega_{M}$ and $\sigma_{8}$ for Three World Models

\begin{tabular}{|c|c|c|c|c|c|c|c|}
\hline & Model & $z$ & Boosts? & $\Omega_{M}$ & Difference & $\sigma_{8}$ & Difference \\
\hline \multirow[t]{12}{*}{$Y$} & \multirow[t]{4}{*}{ Flat } & \multirow[t]{2}{*}{$0,0.5$} & no & 0.287 & & 0.857 & \\
\hline & & & yes & 0.289 & $0.7 \%$ & 0.854 & $-0.4 \%$ \\
\hline & & \multirow[t]{2}{*}{$0,1.0$} & no & 0.277 & & 0.865 & \\
\hline & & & yes & 0.277 & $0.0 \%$ & 0.865 & $0.0 \%$ \\
\hline & \multirow[t]{4}{*}{ Open } & \multirow[t]{2}{*}{$0,0.5$} & no & 0.278 & & 0.857 & \\
\hline & & & yes & 0.279 & $0.4 \%$ & 0.856 & $-0.1 \%$ \\
\hline & & \multirow[t]{2}{*}{$0,1.0$} & no & 0.279 & & 0.855 & \\
\hline & & & yes & 0.280 & $0.4 \%$ & 0.855 & $0.0 \%$ \\
\hline & \multirow[t]{4}{*}{ EdS } & \multirow[t]{2}{*}{$0,0.5$} & no & 0.932 & & 0.531 & \\
\hline & & & yes & 0.931 & $-0.1 \%$ & 0.531 & $0.0 \%$ \\
\hline & & \multirow[t]{2}{*}{$0,1.0$} & no & 0.874 & & 0.541 & \\
\hline & & & yes & 0.873 & $-0.1 \%$ & 0.541 & $0.0 \%$ \\
\hline \multirow[t]{12}{*}{$y_{\max }$} & \multirow[t]{4}{*}{ Flat } & \multirow[t]{2}{*}{$0,0.5$} & no & 0.295 & & 0.844 & \\
\hline & & & yes & 0.199 & $-33 \%$ & 1.020 & $21 \%$ \\
\hline & & \multirow[t]{2}{*}{$0,1.0$} & no & 0.267 & & 0.870 & \\
\hline & & & yes & 0.229 & $-14 \%$ & 0.976 & $12 \%$ \\
\hline & \multirow[t]{4}{*}{ Open } & \multirow[t]{2}{*}{$0,0.5$} & no & 0.282 & & 0.848 & \\
\hline & & & yes & 0.213 & $-24 \%$ & 0.984 & $16 \%$ \\
\hline & & \multirow[t]{2}{*}{$0,1.0$} & no & 0.281 & & 0.848 & \\
\hline & & & yes & 0.236 & $-16 \%$ & 0.954 & $13 \%$ \\
\hline & \multirow[t]{4}{*}{ EdS } & \multirow[t]{2}{*}{$0,0.5$} & no & 0.953 & & 0.524 & \\
\hline & & & yes & 0.921 & $-3.4 \%$ & 0.589 & $12 \%$ \\
\hline & & \multirow[t]{2}{*}{$0,1.0$} & no & 0.905 & & 0.532 & \\
\hline & & & yes & 0.924 & $2.1 \%$ & 0.590 & $11 \%$ \\
\hline
\end{tabular}




\title{
2.6.2 Fitting the Dark Energy Equation of State Parameter
}

\author{
$w$
}

Clusters of galaxies have been used to constrain the equation of state parameter $w$ of dark energy, and there are extensive plans to improve these measurements in the future using SZ surveys (e.g., Haiman et al. 2001; Weller et al. 2002). In the $\Lambda \mathrm{CDM}$ flat world model, dark energy is assumed to take the form of a cosmological constant, which has a fixed $w=-1$. Here, we assess the effect of mergers on the determination of $w$ by allowing $w$ to vary along with $\Omega_{M}$ and $\sigma_{8}$ in fits to the flat world model YFs and yFs, following the same procedure outlined in $\S$ 2.6.1. We need new analytic, nonboosted Press-Schechter YFs and yFs that incorporate $w \neq-1$, which we write as $n_{P S}(Y, z, w)$ and $n_{P S}\left(y_{\max }, z, w\right)$. The same basic form of $n_{P S}$ can be generalized to a constant $w \neq-1$ and a slowly varying parameterization of $w(z)=w_{0}+w_{1} a(1-a)=w_{0}+w_{1} z /(1+z)^{2}$, where $a$ is the scale factor and $w_{0}$ and $w_{1}$ are constants and $w_{1}$ is small. In a flat $\left(\Omega_{M}+\Omega_{D E}=1\right)$ universe, we change the expression for the growth function $D(z)$ as given in Appendix A of RSR and correct the comoving volume element $d V$ such that

$$
\left(\frac{d N}{d V d Y}\right)^{w}=\left(\frac{d N}{d V d Y}\right)^{w=-1, \delta_{c}(D(z, w))}\left(\frac{d_{\mathrm{A}}^{w=-1}}{d_{\mathrm{A}}^{w}}\right)^{2}\left(\frac{d V^{w=-1}}{d V^{w}}\right)
$$

where $\left(\frac{d N}{d V d Y}\right)^{w=-1, \delta_{c}(D(z, w))}$ is $n_{P S}(Y, z)$ for the $\Lambda$ CDM cosmology, but with the critical overdensity $\delta_{c}$ given by the new growth function $D(z, w)$ [equation (20) from Percival (2005) for constant $w$ or equation (14) from Wang \& Steinhardt (1998) for for $w(z)$ ], and $d_{\mathrm{A}}$ is the angular diameter distance. The ratio of volumes is

$$
\left(\frac{d V^{w=-1}}{d V^{w}}\right)=\left(\frac{d_{\mathrm{A}}^{w=-1}}{d_{\mathrm{A}}^{w}}\right)^{2}\left[\frac{E(z, w)}{E(z, w=-1)}\right]
$$


where $E(z, w)=\left[\Omega_{M}(1+z)^{3}+\Omega_{D E}(1+z)^{3+3 w}\right]^{1 / 2}$. The same expression applies for the yFs by replacing $Y$ with $y_{\max }$ and dropping the factor $\left(\frac{d_{\mathrm{A}}^{w=-1}}{d_{\mathrm{A}}^{w}}\right)^{2}$ from equation $(2.14)$.

Constant $w \neq-1$

When we allow for constant values of $w$ that are not necessarily equal to -1 , we find results qualitatively similar to what was found previously when only $\Omega_{M}$ and $\sigma_{8}$ were varied. Again, the boosted YFs give back nearly identical values for all 3 parameters to within $\lesssim 1 \%$. For $y_{\max }$, merger boosts are found to bias the fitted values for $\Omega_{M}$ and $\sigma_{8}$ even more strongly, underestimating $\Omega_{M}$ by $30-45 \%$ and overestimating $\sigma_{8}$ by $20-25 \%$. Also, $w$ is found to be more negative in the boosted yFs by $25-45 \%$, making $y_{\max }$ a poor proxy if one aims to constrain the nature of dark energy. These results are summarized in Table 2.4.

In the case of $y_{\max }$, the boosted yFs favor more negative values of $w$ due to $w$ 's impact on structure formation. The $\mathrm{yF}$ is overestimated to a greater extent at larger redshifts (see Figure 2.6), which mimics more structure in the recent $(z \lesssim 1)$ past. In turn, the appearance of more collapsed structures in the past relative to the present time implies that recent structure formation was slower than it actually has been, and that structure formation in the far past was correspondingly faster. In general, if we compare the effect of different values of $w$ on structure formation by holding the present $\mathrm{yF}$ fixed, a more negative $w$ is better able to slow down cluster formation at later times as the strength of dark energy grows with the scale factor $a$ since $\Omega_{D E}=\Omega_{D E, 0}(1+z)^{3(1+w)} / E^{2}(z)$. If cluster formation is slowing at the current epoch, when dark energy has recently become dominant, there must be more clusters in the recent past compared to the $\mathrm{yF}$ of clusters under the influence of a less negative $w$.

A more negative $w$ allows for even smaller values of $\Omega_{M}$ to be fit to the boosted 
yFs, compared to its best-fit values when only $\Omega_{M}$ and $\sigma_{8}$ are varied. By anchoring the current $\mathrm{yF}$, a more negative $w$ decreases the influence of dark energy in the past, so $\Omega_{M}$ does not need to be as large to form the same amount of structure. The dark energy equation of state does not as directly affect the overall normalization of the $\mathrm{yFs}$, so the bias to $\sigma_{8}$ remains consistent with the fixed $w=-1$ fits.

\section{Slowly Varying $w(z)$}

If dark energy is not due to a cosmological constant, then it is possible that its equation of state might vary. We have also determined the effect that merger boosts can have on the SZ determination of the evolution of dark energy. We only consider the effect of boosted YFs in this section, due to the difficulty of using yFs to pin down even constant values of $w$. Choosing the parameterization of $w=w_{0}+w_{1} z /(1+z)^{2}$, where $w_{0}$ and $w_{1}$ are constants, we determined $\Omega_{M}, \sigma_{8}, w_{0}$, and $w_{1}$ by fitting the boosted and nonboosted YFs. The validity of the form of the growth function we

use for a flat universe requires that $\left|\frac{d w}{d \Omega_{M}}\right| \ll \frac{1}{1-\Omega_{M}}$, which implies that $w_{1} \ll 1$ for $w_{0} \approx-1$ and $\Omega_{M} \approx 0.3$ (Wang \& Steinhardt 1998). We do not constrain our best-fit value of $w_{1}$ according to this requirement, however, nor do we consider any other parameterization of $w$.

We found that $w_{1}$ was not well-constrained by fitting the YFs or yFs at only two redshifts. Thus, we simultaneously fit the distribution functions at the three redshifts $z=0,0.5$, and 1 . As in the constant $w$ case, the boost-derived values of $\Omega_{M}, \sigma_{8}$, and $w_{0}$ deviated from the nonboosted values only slightly, by $+0.5 \%,-0.2 \%$, and $+2 \%$, respectively. The best-fit values of $w_{1}$ increased by 0.15 from the nonboosted value of -0.19 to a value for the boosted YF of -0.04 . For the assumed variation of $w$ with $z$, the largest change in $w$ occurs between the present time $(z=0)$ and $z=1$; 
that change is $\Delta w=w_{1} / 4$. Thus, the merger boost effects on YF alter the maximum change in the $w$ by about 0.04 . This is about twice as large as the effect on $w_{0}$, but is still relatively small.

\subsection{Discussion and Summary}

We have determined the effects of cluster mergers on their SZ properties, particularly the integrated $Y$ and maximum $y_{\max }$ Comptonization parameters. From a set of hydrodynamical/N-body simulations of cluster mergers, we determined the evolution of $Y$ and $y_{\max }$ over the period of interaction for mergers of various mass ratios and impact parameters, and we found that mergers temporarily "boost" both $Y$ and $y_{\max }$. For $y_{\max }$, the boosts can be as large as an order of magnitude, although they occur for a short time (typically about half the sound crossing time of the cluster), with the largest boosts occurring near the time of first core crossing. For major mergers, the boosts in the maximum Comptonization parameter generally exceed the increase in $y_{\max }$ when the systems have come into equilibrium.

On the other hand, the boosts in $Y$ are smaller (less than a factor of two), although they last longer (about two sound crossing times). Most importantly, the boosts in $Y$ for major mergers are smaller than the increases in $Y$ when the merged clusters have come into equilibrium. Thus, one can think of the merger "boost" in $Y$ as representing a stage in the evolution from two separate equilibrium values to the final merged value, and not really being a "boost" at all. A simple physical argument explains why the transient boosted values of $Y$ are smaller than the final equilibrium values. From equation (2.2), it follows that $Y$ is just proportional to the total thermal energy content of the electrons in the clusters, or just the total thermal energy if the electrons and ions are in equipartition. Now, a cluster merger involves the conversion 
of the bulk kinetic energy of the merging clusters into thermal energy. When the merger is complete, there is very little bulk kinetic energy remaining (perhaps, weak rotation or turbulence). Thus, one expects the thermal energy content of the merging clusters to be largest when they have achieved (or nearly achieved) equilibrium. Thus, the final equilibrium value of $Y$ will tend to be larger than any transient value during the merger.

We generalized the SZ boosts to mergers of arbitrary mass ratio and impact parameter and traced the merger, and thus boost, history of clusters with redshift using the EPS merger tree formalism. In general, merger boosts induced a relatively small scatter, $\sim 2 \%$, below the equilibrium $Y-M$ relation, while mergers induced a large scatter of $25-30 \%$ above the $y_{\max }-M$ equilibrium relation. Power-law fits to $Y$ and $y_{\max }$ as a function of mass show that while boosts do not affect the slope of the fit, the normalization was lowered by $<1 \%$ for $Y$ and raised by $\sim 10 \%$ for $y_{\max }$.

We used the merger trees to derive the distribution functions of SZ parameters, YF and $y F$. We found that the boosted YF was not significantly biased relative to the nonboosted YF, while the boosted yF was strongly biased above the nonboosted $\mathrm{yF}$ for all redshifts. In general, the size of the merger-induced bias increased with redshift and with cluster mass.

Using the YFs and $y F s$, we determined the best-fit values for the cosmological parameters $\Omega_{M}$ and $\sigma_{8}$ for the flat, open, and EdS world models, and also the dark energy equation of state parameter $w$ for the flat universe. Comparing the best-fit values of $\Omega_{M}$ and $\sigma_{8}$ for the nonboosted and boosted YF, no significant difference $(<1 \%)$ was observed. In contrast, the boosts to the $\mathrm{yF}$ decreased the best-fit value of $\Omega_{M}$ by $15-30 \%$ for the flat and open world models and increased the best-fit value of $\sigma_{8}$ by $10-20 \%$ for all world models. These results stem mainly from an overall increase 
in the yFs, which pushes $\sigma_{8}$ to larger values, and a greater increase in the boosted yF at higher redshifts relative to lower redshifts, which pushes $\Omega_{M}$ to smaller values. Allowing for a constant $w \neq-1$ in the flat world model, no systematic difference in fitted cosmological parameters was found between the two sets of YFs, though the merger-induced bias to $\Omega_{M}, \sigma_{8}$, and $w$ was exacerbated when using the yFs. We also considered a time-varying $w(z)$ for the YFs, for which $\Omega_{M}, \sigma_{8}$, and $w_{0}$ were found to be consistent with the previous results for a constant $w \neq-1$, though boosts increased the best-fit value of the dark energy evolution parameter $w_{1}$ by about 0.15 . The largest change in $w$ occurs between $z=1$ and $z=0$ in this model; thus, the change in $w$ might be affected by as much as 0.04 . This is about twice as large as the maximum change in the present-day value of $w_{0}$, but still is relatively moderate.

These results agree with previous work which indicates that global observables such as $Y$ or the equivalent $\mathrm{X}$-ray/mass proxy $Y_{X}$ are more robust as mass proxies than the central or maximum Comptonization parameter. For example, from semianalytic models of the intracluster medium (ICM), Reid \& Spergel (2006) generally find that $Y \propto f_{g} M^{5 / 3}$, equivalent to our equilibrium definition of $Y$, with only a small scatter due to internal physics. A number of studies have used cosmological Nbody simulations and re-simulated forming clusters with various kinds of gas physics to evaluate the scatter in the $y-M$ relations (Nagai 2006; Bonaldi et al. 2007). It is generally found that the normalization $A$ varies significantly depending on the ICM physics, though the slope $\alpha$ does not. Nagai (2006) reports a scatter of 10$15 \%$ in the $Y-M$ relation, much larger than our scatter of $\sim 2-3 \%$. Also, Kravtsov et al. (2006) defines an X-ray observable $Y_{X}=T_{X} M_{\text {gas }, 500}$, which is similar to our $Y \propto \int n_{e} T_{e} d V \sim T_{e} \int n_{e} d V \propto T_{e} M_{\text {gas }}$; they find a scatter in the relation of $5 \%-7 \%$.

While these studies intrinsically include mergers, they have limited statistics as 
they generally consist only of a small number of systems, $\sim 10$ or so. Some studies have considered somewhat larger cluster simulation samples including hundreds of clusters (Motl et al. 2005; da Silva et al. 2004) from cosmological simulations. Our results agree with their conclusions that the $Y-M$ relation is relatively stable to mergers, unlike the $y_{\max }-M$ relation. Motl et al. (2005) find a scatter in their $Y-M$ relation of $3-4 \%$ and in their $y_{\max }-M$ relation of $\sim 17 \%$ at $z=0$ due to mergers and other ICM physical processes. These results compare well with our scatter of $2 \%$ and $24 \%$, respectively.

In a study similar to this work, Poole et al. (2007) take a suite of binary cluster merger simulations to assess the effect of various observables, including SZ parameters, on scaling relations during mergers. The evolution of $y_{\max }$ (which they call $y_{0}$ ) in their simulations is qualitatively similar to our results in Figure 2.2 for various impact parameters and mass ratios. They also consider an integrated Comptonization parameter, but it is only integrated out to a radius $r_{2500}$ and is thus much more dominated by core effects and not equivalent to our $Y$, which is effectively integrated to at least $r_{200}$, the virial radius.

The large number of galaxy clusters expected from upcoming SZ surveys, both locally and at potentially high redshifts, heightens the prospects that clusters could play a decisive role in the era of precision cosmology, especially if the robustness of $Y$ as a proxy for mass is confirmed in real cluster samples. 
Table 2.4. Best-Fit Flat World Models with Constant $w$

\begin{tabular}{|c|c|c|c|c|c|c|c|c|}
\hline & $z$ & Boosts? & $\Omega_{M}$ & Difference & $\sigma_{8}$ & Difference & $w$ & Difference \\
\hline \multirow[t]{4}{*}{$Y$} & $0,0.5$ & no & 0.314 & & 0.837 & & -0.879 & \\
\hline & & yes & 0.316 & $0.6 \%$ & 0.835 & $-0.2 \%$ & -0.885 & $0.7 \%$ \\
\hline & $0,1.0$ & no & 0.275 & & 0.874 & & -1.062 & \\
\hline & & yes & 0.271 & $-1.5 \%$ & 0.877 & $0.3 \%$ & -1.080 & $1.7 \%$ \\
\hline \multirow[t]{4}{*}{$y_{\max }$} & $0,0.5$ & no & 0.324 & & 0.823 & & -0.861 & \\
\hline & & yes & 0.173 & $-47 \%$ & 1.082 & $24 \%$ & -1.255 & $46 \%$ \\
\hline & $0,1.0$ & no & 0.279 & & 0.863 & & -0.987 & \\
\hline & & yes & 0.192 & $-31 \%$ & 1.045 & $21 \%$ & -1.240 & $26 \%$ \\
\hline
\end{tabular}




\section{Chapter 3}

\section{A Suzaku Search for Nonthermal Emission at Hard X-ray Energies in the Coma Cluster ${ }^{1}$}

\section{Abstract}

The brightest cluster radio halo known resides in the Coma cluster of galaxies. The relativistic electrons producing this diffuse synchrotron emission should also produce inverse Compton emission that becomes competitive with thermal emission from the ICM at hard X-ray energies. Thus far, claimed detections of this emission in Coma are controversial (e.g., Fusco-Femiano et al. 2004; Rossetti \& Molendi 2004). We present a Suzaku HXD-PIN observation of the Coma cluster in order to nail down its nonthermal hard X-ray content. The contribution of thermal emission to the HXD-PIN spectrum is constrained by simultaneously fitting thermal and nonthermal models to it and

\footnotetext{
${ }^{1}$ Published: Wik, D. R., Sarazin, C. L., Finoguenov, A., Matsushita, K., Nakazawa, K., \& Clarke, T. E. 2009, ApJ, 696, 1700
} 
a spatially equivalent spectrum derived from an XMM-Newton mosaic of the Coma field (Schuecker et al. 2004). We fail to find statistically significant evidence for nonthermal emission in the spectra, which are better described by only a single or multi-temperature model for the ICM. Including systematic uncertainties, we derive a

$90 \%$ upper limit on the flux of nonthermal emission of $6.0 \times 10^{-12} \mathrm{erg} \mathrm{s}^{-1} \mathrm{~cm}^{-2}(20-80$ $\mathrm{keV}$, for $\Gamma=2.0$ ), which implies a lower limit on the cluster-averaged magnetic field of $B>0.15 \mu \mathrm{G}$. Our flux upper limit is $2.5 \times$ lower than the detected nonthermal flux from RXTE (Rephaeli \& Gruber 2002) and BeppoSAX (Fusco-Femiano et al. 2004). However, if the nonthermal hard X-ray emission in Coma is more spatially extended then the observed radio halo, the Suzaku HXD-PIN may miss some fraction of the emission. A detailed investigation indicates that $\sim 50-67 \%$ of the emission might go undetected, which could make our limit consistent with Rephaeli \& Gruber (2002) and Fusco-Femiano et al. (2004). The thermal interpretation of the hard Coma spectrum is consistent with recent analyses of INTEGRAL (Eckert et al. 2007a) and Swift (Ajello et al. 2009) data.

\subsection{Introduction}

In the hierarchical scenario of cosmic structure formation, clusters of galaxies form at late times through mergers between subclusters and through the accretion of galaxies and galaxy groups. The distribution of their massive halos in space and time depend sensitively on the underlying cosmology, and much effort has been made to connect observable properties of the gas to the total cluster mass in order to constrain cosmological parameters (e.g., Mantz et al. 2008, and references therein). However, merger processes are known to significantly disrupt the thermal gas (e.g. Ricker \& Sarazin 2001; Ritchie \& Thomas 2002), typically biasing inferred masses and the 
resultant cosmological parameter estimates (Randall et al. 2002; Wik et al. 2008). Merger-induced shocks and turbulence, besides heating the gas, are thought to also re-accelerate relativistic particles present in the intracluster medium (ICM) (Sarazin 1999; Brunetti \& Blasi 2005). Nonthermal electrons, observed via diffuse, radio synchrotron emission, have been detected in over 50 clusters, all of them undergoing mergers (Buote 2001; Schuecker et al. 2001). If the energy in a relativistic phase of the ICM is large enough to add pressure support to the thermal gas, even transiently, the ability to derive masses and therefore use clusters as cosmological probes may be compromised (Skillman et al. 2008). An assessment of the relativistic contribution to the energy budget of clusters is necessary to fully characterize the state of the ICM.

Diffuse, cluster-wide synchrotron radio emission, called radio halos or relics depending on their morphology, imply that both magnetic fields and relativistic electron populations are present on large scales. The total luminosity of a synchrotronemitting electron is given by

$$
L_{R}=\frac{4}{3} \sigma_{T} c \gamma^{2} \epsilon_{B}
$$

where $\sigma_{T}$ is the Thomson cross-section, $c$ is the speed of light, $\gamma$ is the Lorentz factor of the electron, and $\epsilon_{B}=B^{2} / 8 \pi$ is the energy density of the magnetic field. For a population of such electrons with various values of $\gamma$, the value of $L_{R}$ depends both on the number of electrons and on $B$ and cannot independently determine either. However, these same electrons will up-scatter cosmic microwave background (CMB) photons through inverse Compton (IC) interactions, which have a luminosity $L_{X}$ equivalent in form to equation (3.1) but with $\epsilon_{B}$ replaced by the energy density of the CMB. Since both luminosities are proportional to the number of electrons, their 
ratio gives the volume-averaged magnetic field,

$$
\frac{L_{R}}{L_{X}}=\frac{B^{2} / 8 \pi}{a T_{C M B}^{4}}
$$

where $a$ is the radiation constant and $T_{C M B}$ is the temperature of the CMB. The IC radiation should be observable at hard X-ray energies (Rephaeli 1977). Thus far, IC emission has only been detected at low significance (Nevalainen et al. 2004) or, in one case, in a cluster with uncertain radio emission (Eckert et al. 2008; but see also Ajello et al. 2009 and Fujita et al. 2008). The measurement of an IC flux from a synchrotron source directly leads to a simultaneous determination of the average value of $B$ and the relativistic electron density (Harris \& Romanishin 1974; Sarazin 1988). Therefore searches for IC emission coincident with a radio halo or relic are an excellent way to constrain the contribution of relativistic materials in clusters.

The first, and brightest, radio halo was discovered by Willson (1970) in the Coma cluster, and its radio properties have perhaps been the best studied (e.g. Giovannini et al. 1993; Deiss et al. 1997; Thierbach et al. 2003). Coma has been observed by all the major observatories with hard X-ray capabilities (Rephaeli et al. 1994; Hughes et al. 1993; Bazzano et al. 1990; Henriksen \& Mushotzky 1986), and more recently nonthermal detections have been claimed by Rephaeli \& Gruber (2002) with RXTE and by Fusco-Femiano et al. $(1999,2004)$ with BeppoSAX, though the latter detection is controversial (Rossetti \& Molendi 2004; Fusco-Femiano et al. 2007). Due to the large field of view (FOV) of these non-imaging instruments and the simple characterization of the thermal gas, the source of this emission remains uncertain. Even more recently, long ( $1 \mathrm{Msec})$ observations with INTEGRAL have imaged extended diffuse hard X-ray emission from Coma, though it was found to be completely consistent with thermal emission (Renaud et al. 2006a; Eckert et al. 2007a; Lutovinov 
et al. 2008).

In this study, we present a Suzaku HXD-PIN observation of the Coma cluster in an effort to detect nonthermal emission associated with the radio halo and potentially confirm the $R X T E$ and BeppoSAX detections. The HXD-PIN instrument has a non-imaging collimator like those on-board $R X T E$ and BeppoSAX, but with a FOV about a quarter as large, which reduces the possible contamination from hard point sources (Takahashi et al. 2007). Also, the Suzaku particle background is $\sim 5 \times$ lower than the backgrounds of either RXTE or BeppoSAX (Mitsuda et al. 2007). In order to clearly distinguish the thermal and nonthermal emission visible within the PIN, the hard Suzaku PIN spectrum is jointly fit with a spatially equivalent XMM-Newton EPIC-pn spectrum. The $X M M$ spectrum, at lower energies and completely dominated by thermal emission, allows Coma's thermal and nonthermal properties to be simultaneously determined. The $X M M$ and HXD-PIN observations are reported in $\S 3.2$ and the extraction of the resulting spectra is discussed in $\S 3.3$. Fits to the joint spectra are described in $\S 3.4$. In $\S 3.5$, we discuss the implications of our results for the nature of the hard X-ray emission from the Coma cluster. We assume a flat cosmology with $\Omega_{M}=0.23$ and $H_{0}=72 \mathrm{~km} / \mathrm{s} / \mathrm{Mpc}$ and a luminosity distance to Coma of $98.4 \mathrm{Mpc}$. Unless otherwise stated, all uncertainties are given at the $90 \%$ confidence level.

\subsection{Observations}

The Suzaku observation was undertaken as part of AO-1 from 2006 May 31 through June 4, soon after 16 of the 64 PIN diode bias voltages were lowered from $500 \mathrm{~V}$ to 400V, but before an additional 16 diodes were similarly lowered. We analyze Version 2 of the pre-processed data (PROCVER 2.0.6.13), which allows for the diode bias 
drop, with HEAsoft 6.4.0 and XSPEC 12.4.0w. For the HXD-PIN instrument, the standard data selection criteria are applied to extract the source spectrum, and the same criteria are used to select times for the modeled non-X-ray background (NXB) spectrum. Specifically, we select observing times when the geomagnetic cut-off rigidity is above a critical value $(\mathrm{COR}>6 \mathrm{GV})$, when the satellite is not within the South Atlantic Anomaly $($ SAA_HXD $=0)$ or has just left it (T_SAA_HXD > 500 s), and when Suzaku is pointed above and at least 5 degrees away from the Earth's horizon $\left(\mathrm{ELV}>5^{\circ}\right)$. The strength of the NXB is known to be roughly inversely proportional to the value of the COR and to be elevated inside the SAA, gradually decaying to typical levels after SAA passage. These criteria ensure that the low NXB of the HXD is minimized and can be well characterized, which is necessary if it is to be accurately modeled. After event selection, the PIN exposure time is reduced from $166.2 \mathrm{ks}$ to $156.1 \mathrm{ks}$ after dead-time correction. The HXD-GSO spectrum is found to be consistent with the background, so we do not consider it further here. We use the Suzaku CCD data from the XIS0 chip to check the cross-calibration of Suzaku and XMM-Newton. Standard event selection was applied to the XIS0 data, leading to an exposure time of $178.7 \mathrm{ks}$.

The mosaic XMM-Newton observations of the Coma cluster, including 14 separate pointings, were done as a part of an instrument performance verification program, a complete log of which is presented in Schuecker et al. (2004). The initial observations were undertaken by and first reported in Briel et al. (2001). Seven new observations, aimed at resolving the temperature structure of the Coma center, have also been performed (PI P. Schuecker). However, high solar activity during the exposures resulted in a high detector background above $2 \mathrm{keV}$, making these observations less suitable for our purposes, and therefore we use only the observations reported in Section 4 and 
Table 2 of Schuecker et al. (2004). We choose only to include the EPIC-pn data from $X M M$ in our analysis. Because these observations were made early in the mission, they cannot be processed with the standard software, though the EPIC-pn data have undergone in-house processing. Also, its effective area at high energies is higher than for the EPIC-MOS detector, making it the more suitable instrument. The benefit of including the EPIC-MOS data is unclear, due to the addition of cross-calibration errors and given the already high signal-to-noise of the EPIC-pn data.

\subsection{Extraction and Construction of Spectra}

To produce complementary spectra from the XMM EPIC-pn and Suzaku HXD-PIN data that can be simultaneously fit, the background and responses of both instruments must be carefully considered to minimize systematic uncertainties. The expected nonthermal signal is near the limit of the PIN sensitivity, and a robust characterization of this emission particularly depends on the accuracy of the PIN background and XMM-Suzaku cross-normalization.

\subsubsection{HXD-PIN Spectrum and Non-X-ray Background}

As the HXD is a non-imaging instrument, we simply extract the PIN spectrum from the selected events and group the spectral bins so that each bin contains at least 30 counts to ensure that Gaussian statistics and $\chi^{2}$ fitting are valid. The response matrix is provided in the Suzaku $\mathrm{CALDB}^{2}$ for Version 2 data products, and we use ae_hxd_pinhxnome2_20080129.rsp for all source components other than the cosmic X-

$\begin{array}{llll}\text { ray } & \text { background } & \text { for } & \text { which }\end{array}$ ae_hxd_pinflate2_20080129.rsp is used.

\footnotetext{
${ }^{2}$ http://suzaku.gsfc.nasa.gov/docs/heasarc/caldb/suzaku/
} 
The non-X-ray background for a PIN observation is most accurately obtained from a model, as opposed to a comparable blank field observation. This method is motivated by the strong dependence of the background count rate and spectral shape on the value of the geomagnetic cut-off rigidity (COR) and the time since the passage of the satellite through the South Atlantic Anomaly (SAA), quantities which vary and have a unique distribution for every observation. The model matches the distribution of the COR and other parameters of the observed data. A model is also required because there is no concurrent measurement of the NXB, such as by nodding between Coma and a blank field. We use the so-called "bgd_d" model for Version 2 processed data, which makes use of HXD-GSO information as well as the COR and SAA values. This NXB model is shown with the PIN data spectrum, uncorrected for the background, in Figure 3.1. While the shape of the NXB is generally well reproduced, the success of the model in determining its overall normalization is $\pm 2.3 \%$ from 15-40 keV and $\pm 4 \%$ from 40-70 keV (Mizuno et al., Suzaku Memo 2008-03³). These estimates of the systematic error in the NXB are extrapolated to the $90 \%$ confidence interval from the $1 \sigma$ values derived using Earth-occulted data in Suzaku Memo 2008-03. We adopt these values (2.3\% from $12-40 \mathrm{keV}$ and $4 \%$ from $40-70$ $\mathrm{keV}$ ) as our estimate of the $90 \%$ systematic error in the PIN NXB. To confirm the accuracy of the model background, we extracted events for both the data and model for times when the Earth occults the PIN FOV $\left(\mathrm{ELV}<-5^{\circ}\right.$, all other selection criteria unchanged); the resulting spectra are shown in Figure 3.2. The fractional difference between the model NXB count rate and the Earth-occulted data is $(0.2 \pm 1.0) \%$ over the energies $12-40 \mathrm{keV}$ and $(-2.0 \pm 2.6) \%$ from $40-70 \mathrm{keV}$ ( $1 \sigma$ errors). Over the whole range considered, the fractional difference in count rates is extremely small: $(0.005 \pm 0.9) \%$. Because of the excellent agreement, we do not adjust the level of the

\footnotetext{
${ }^{3}$ http://www.astro.isas.ac.jp/suzaku/doc/suzakumemo/suzakumemo-2008-03.pdf
} 
background as proposed in Ishida et al., Suzaku Memo 2007-104.

The estimate of the systematic error adopted here comes from an analysis of Earth-occulted data, which is the same data used to generate models of the NXB. However, it is possible that a systematic effect could be present during observations of the sky that would not exist during Earth-occulted observations, and so it would not be included in the NXB model or the estimate of the systematic uncertainty. Mizuno et al. (Suzaku Memo 2008-03) attempt to test for this possibility with "blank sky" observations and find a larger effective systematic uncertainty. It is clear that some part of this uncertainty is due to the fact that the fields aren't entirely "blank" and that the source flux will vary field-to-field. Here we refer to relatively bright sources not considered to be part of the more uniform $\mathrm{CXB}$, which has a variance based on the XIS sensitivity to point sources that can be taken into account. When considering many observations of one region on the sky, so that the contamination from sources will vary less, the standard deviation drops from $5.8 \%$ derived from many fields to $5.0 \%$, both of which includes a statistical error of about $3.3 \%$. While an additional systematic uncertainty, only in effect when observing the sky, cannot be ruled out, this drop suggests that systematic error estimates derived from sky observations are somewhat conservative. Because the contribution of contaminating sources to systematic error estimates is thus far not well-characterized, we use the value derived from Earth-occulted observations throughout to avoid overestimating this error. We rely on the assumption that a full accounting of contaminating sources would lead to a systematic error estimate similar to our adopted value. However, using the sample of 10 ks exposures of all blank sky observations leads to an estimate of the NXB systematic error of $4 \%$, after subtracting the statistical error and the expected CXB fluctuation (see e.g. Nakazawa et al. 2009). We consider the effect

\footnotetext{
${ }^{4}$ http://www.astro.isas.ac.jp/suzaku/doc/suzakumemo/suzakumemo-2007-10.pdf
} 


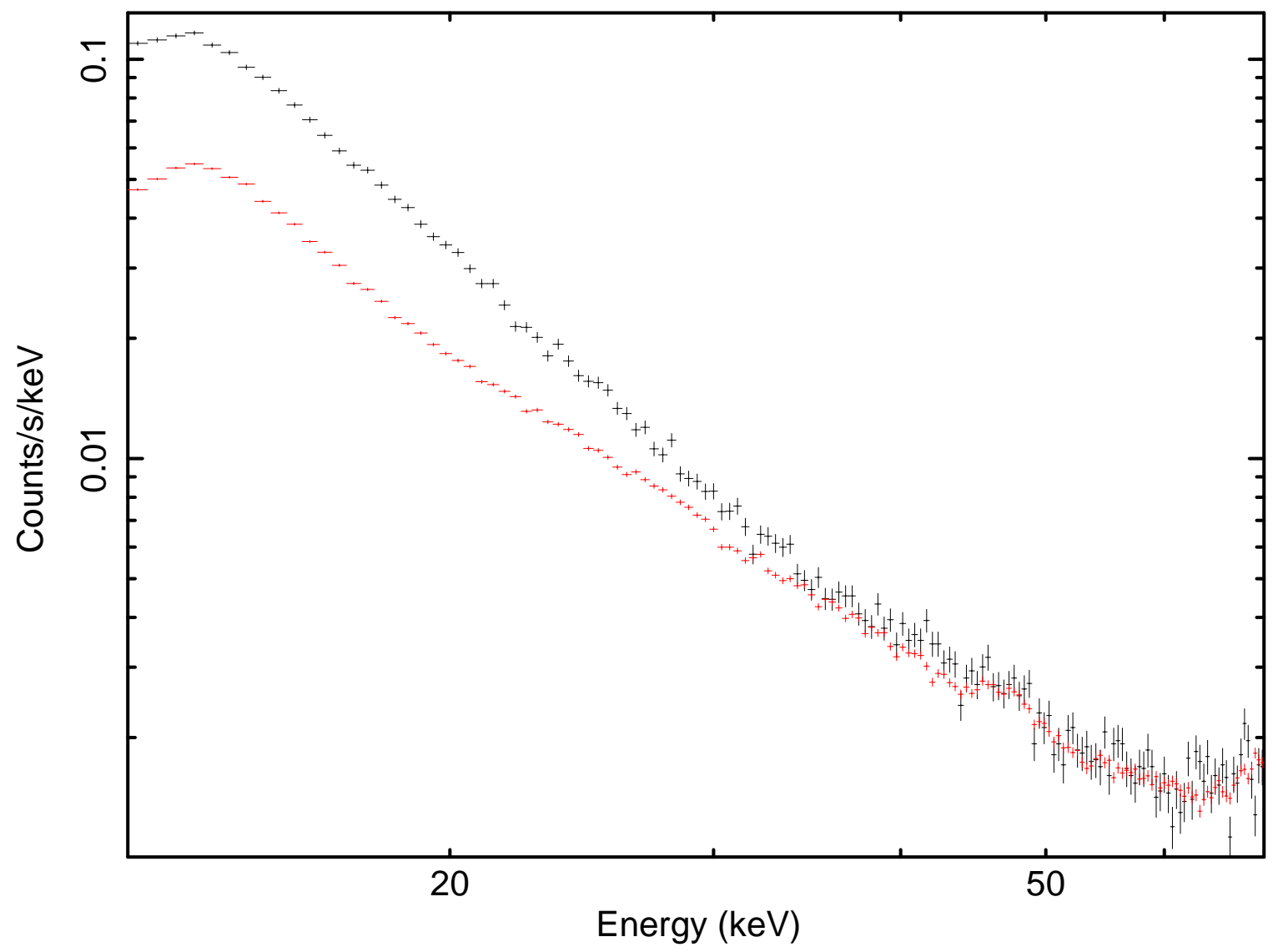

Fig. 3.1.- Suzaku HXD-PIN NXB model spectrum (red data points) compared to the Coma cluster data (uncorrected for background). Note that at energies above 45 $\mathrm{keV}$, the NXB dominates the data and that deviations of the data above the NXB are confined to individual channels that are simply statistical fluctuations or are otherwise imperfectly characterized by the NXB model. 


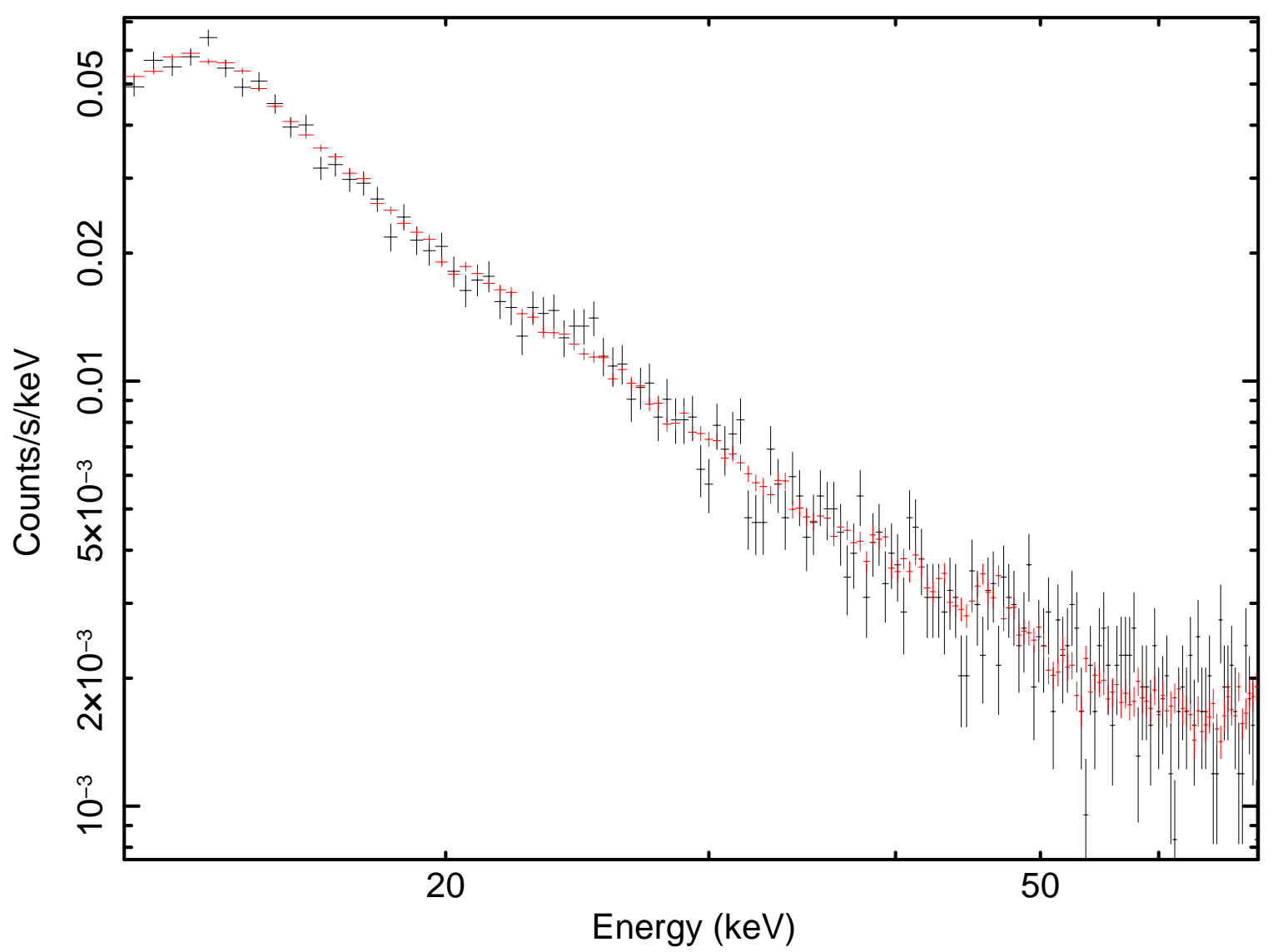

Fig. 3.2.- Suzaku HXD-PIN Earth-occulted data (black data points) compared to the NXB model spectrum for the same time periods of Earth-occultation (red data points). During Earth-occultation, the only events should be due to the NXB. Note that the NXB agrees well with the normalization and shape of the Earth-occulted data at all energies $\gtrsim 12 \mathrm{keV}$. (Only this range is used in the spectral fits for Coma.) 
of raising the systematic error to this higher value (for $E<40 \mathrm{keV}$ ) in $\S 3.4 .3$; our results and conclusions remain qualitatively unchanged.

\subsection{2 $\quad X M M$ EPIC-pn Spectrum}

To constrain the thermal contribution to the PIN hard X-ray spectrum, it is very helpful to have a spectrum for the same region covered by the HXD-PIN FOV but extending to lower energies where the thermal emission is completely dominant. This low energy spectrum acts as a lever arm on the thermal continuum so that the properties of the thermal gas can be extracted simultaneously with a potential nonthermal component. Because the ICM of Coma is not isothermal and its projected temperature varies across the cluster, a complimentary spectrum at softer energies must follow the spatial sensitivity of Suzaku's HXD. Otherwise, localized regions of even slightly hotter gas could mimic the emission of a nonthermal source at hard energies. Since the HXD is made up of 64 individual collimators with optical axes generally aligned to within $4^{\prime}$ of each other, we approximate the PIN spatial response as a single perfect collimator with a total square FOV of $D=65^{\prime} .5$ on a side,

$$
R_{\text {coll }}=\left(D / 2-\theta_{x}\right)\left(D / 2-\theta_{y}\right) /(D / 2)^{2}
$$

where $R_{\text {coll }}$ is the fraction of the flux detected at angles of $\left(\theta_{x}, \theta_{y}\right)$ from the optical axis along the PIN detector axes, relative to a point source located at the center of the $\operatorname{HXD} \operatorname{FOV}\left(\theta_{x}=\theta_{y}=0\right)$. We have verified that this model fits the spatial response of the PIN very well. The complimentary $X M M$ spectrum is constructed based on this spatial vignetting of the PIN, which is reasonable for our energy range of interest $(<70 \mathrm{keV})$.

In order to build a spectrum that reflects the PIN vignetting with good statis- 
tics, we extract spectra from 10 regions of roughly equal effective area, as shown in Figure 3.3. The boundaries of the regions are spaced at intervals of $10 \%$ of the PIN sensitivity to a central point source. Because the solid angle subtended by a region increases with its distance from the cluster center, it turns out that the count rates of each of these $X M M$ spectra are comparable. The same response matrix is used for all spectra, epn_ef20_sdY7_medium.rsp, and the auxiliary response files (arfs) for each region are generated in the standard way (Lumb et al. 2003). The background spectrum is derived from the datasets compiled by Read \& Ponman (2003), to which we also apply consistent flare cleaning criteria. Before summing these spectra, weighted by the average PIN sensitivity within each region, we scale the arfs so they all agree with the central region (R10) arf at $5 \mathrm{keV}$, while also scaling the exposure times so the flux remains unchanged. Similarly normalized arfs are required to ensure that the weighted and summed arf will properly represent the response of the final summed spectrum.

We now describe the procedure for creating the summed observed rate spectrum, summed background spectrum, and corresponding response. Let $O_{j}^{i}$ be the observed rate spectrum in spectral channel $j$ for region $i$, and let $B_{j}^{i}$ be the corresponding background spectrum. We constructed the weighted sums

$$
\begin{aligned}
& \bar{O}_{j}=\sum_{i=1}^{10} w^{i} O_{j}^{i}, \\
& \bar{B}_{j}=\sum_{i=1}^{10} w^{i} B_{j}^{i},
\end{aligned}
$$

where $w^{i}$ is the weight of region $i$ in the PIN spectrum, based on the average value of equation (3.3) inside the region (Table 3.1), normalized by the PIN nominal central point source sensitivity. Let $R_{j k}^{i}$ be the response matrix for region $i$, defined such 


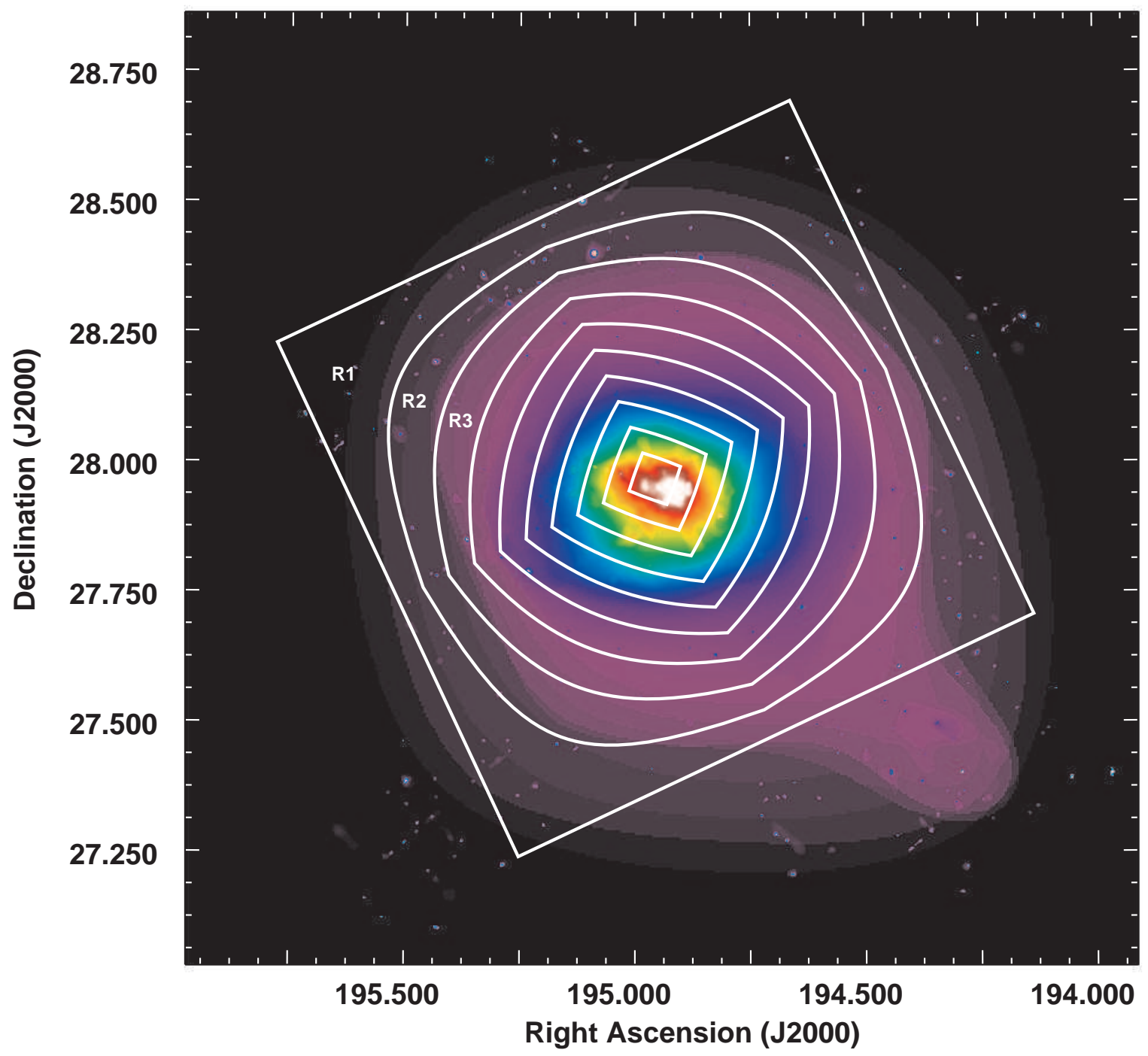

Fig. 3.3.- XMM EPIC-pn 2-7.5 keV wavelet smoothed X-ray surface brightness image (Schuecker et al. 2004) with contours of constant Suzaku HXD-PIN effective area overlaid. The contours are spaced at $10 \%$ intervals running from $0 \%$ to $90 \%$ of the effective area for a point source located at the instrument center. In between the contours, the regions labeled R1, R2, etc., correspond to those in Table 3.1 and in the text. 
that given a number flux $M_{k}^{i}$ of photons per unit area and time at Earth in spectral channel $k, R_{j k}^{i} M_{k}^{i}$ is the number of events per second observed in spectral channel $j$. In the nomenclature of X-ray spectral analysis, $R_{j k}^{i}$ is the "rsp" file for region $i$. The corresponding average response matrix, $\bar{R}_{j k}$, must be weighted both by the PIN effective area for the regions (the weights $w_{i}$ ) and by the number of $X M M$ photons incident on each area. To include the weighting by the incident flux on each area, we formally assume that to first order the spectra in all 10 regions are described by models $M_{k}^{i}$ that have identical spectral shapes given by $m_{k}$, but differing normalizations $N^{i}$. That is, $M_{k}^{i}=N^{i} m_{k}$. In our case, we take $m_{k}$ as an APEC model with $T=8.2 \mathrm{keV}$ and abundance relative to solar of 0.24 , which is the best fit model to the XMM-Newton EPIC-pn spectrum for the entire Suzaku PIN FOV. While the temperatures from the outer 5 regions (R5-R1, Table 3.1) are somewhat inconsistent with our fiducial $T$, the central regions are weighted more strongly and so it is more important to accurately match their spectral shape than that of the spectra from R5-R1. The average of the temperatures from $\mathrm{R} 10-\mathrm{R} 1$ is in good agreement with previous measurements of Coma's global temperature (Watanabe et al. 1999; Arnaud et al. 2001). Also, temperatures in R10, R9, and R8 are just consistent at the $90 \%$ level with continuum and Fe line ratio fits to the XIS data extracted from those regions, using the method described in Sato et al. (2009). The models $M_{k}^{i}$ now differ only in overall flux, given by the APEC normalization $N^{i}$, so each observed spectrum can be described as

$$
O_{j}^{i}-B_{j}^{i}=\sum_{k} R_{j k}^{i} M_{k}^{i}=N^{i} \sum_{k} R_{j k}^{i} m_{k} .
$$


Similarly, we define $\bar{R}_{j k}$ as

$$
\bar{O}_{j}-\bar{B}_{j}=N \sum_{k} \bar{R}_{j k} m_{k}
$$

where $N$ is the APEC normalization of the summed spectrum. Substituting equations (3.4) and (3.5) into equation (3.7) yields, after some algebra,

$$
\sum_{k} \bar{R}_{j k} m_{k}=\sum_{k} m_{k} \sum_{i} w^{i} \frac{N^{i}}{N} R_{j k}^{i}
$$

so it is clear that

$$
\bar{R}_{j k}=\sum_{i=1}^{10} w^{i} \frac{N^{i}}{N} R_{j k}^{i} .
$$

The value of the weighted normalization $N$ is given by $N=\sum_{i} w^{i} N^{i}$.

For all fits of the $X M M$ spectra, the energy range considered differed slightly from the nominal 2-12 keV energy range due to calibration issues. At energies near $\sim 2$ $\mathrm{keV}$, there exists a sharp edge in the response due to gold in the mirrors, and between 8 and $9 \mathrm{keV}$ there are variable background lines due to copper and zinc. Neither of these features can be sufficiently accounted for given the current calibration, and they tend to become important in regions with very good statistics (all of the Coma $X M M$ spectra) and/or regions of low surface brightness particularly near the outer edges of the detector. We avoid these problems by excluding these features and only fitting over the range $2.3<E<7.5 \mathrm{keV}$ and $9.5<E<12 \mathrm{keV}$. Also, the gain can vary by up to $30 \mathrm{eV}$, especially during the period when many of these observations took place (Marcus Kirsch, XMM Calibration Document ${ }^{5}$ ) and the redshift found from fits differs significantly from the nominal value of $z=0.023$. We fit the gain with a linear function, assuming the redshift to be 0.0232, using the GAIN command in

\footnotetext{
${ }^{5}$ http://xmm2.esac.esa.int/docs/documents/CAL-TN-0018.pdf
} 
Table 3.1. $\quad X M M$ Regions and Spectral Fits

\begin{tabular}{lccc}
\hline \hline Region & PIN Weight & $\begin{array}{c}k T \\
(\mathrm{keV})\end{array}$ & $\begin{array}{c}\text { Norm. }^{\mathrm{a}} \\
\left(10^{-2} \mathrm{~cm}^{-5}\right)\end{array}$ \\
\hline R10 & 0.933 & $8.25 \pm 0.21$ & $1.67 \pm 0.01$ \\
R9 & 0.844 & $8.33 \pm 0.14$ & $4.25 \pm 0.02$ \\
R8 & 0.746 & $8.27 \pm 0.15$ & $5.17 \pm 0.03$ \\
R7 & 0.647 & $8.07 \pm 0.17$ & $5.09 \pm 0.03$ \\
R6 & 0.547 & $8.07 \pm 0.23$ & $4.52 \pm 0.04$ \\
R5 & 0.448 & $7.40 \pm 0.34$ & $3.65 \pm 0.05$ \\
R4 & 0.348 & $7.39 \pm 0.46$ & $2.93 \pm 0.05$ \\
R3 & 0.248 & $6.99 \pm 0.56$ & $2.84 \pm 0.06$ \\
R2 & 0.147 & $7.65 \pm 0.44$ & $3.30 \pm 0.05$ \\
R1 & 0.0421 & $7.45 \pm 0.68$ & $2.76 \pm 0.06$ \\
\hline
\end{tabular}

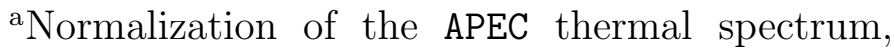
which is given by $\left\{10^{-14} /\left[4 \pi(1+z)^{2} D_{A}^{2}\right]\right\} \int n_{e} n_{H} d V$, where $z$ is the redshift, $D_{A}$ is the angular diameter distance, $n_{e}$ is the electron density, $n_{H}$ is the ionized hydrogen density, and $V$ is the volume of the cluster. 
XSPEC for all 10 spectra. We adjusted the gain such that the new response energies $E^{\prime}$ are related to the original energies by $E^{\prime}=E / 1.00519+0.010312$. None of these calibration issues have any important effect on the characterization of the continuum features (IC and hard thermal bremsstrahlung) which are the subject of this paper. However, not including these effects would result in high values of $\chi^{2}$ for the fits due to the very good statistics in the Coma $X M M$ EPIC-pn spectra, and thus make it more difficult to determine the uncertainties in parameters.

Even after these adjustments, fits to the weighted and summed final spectrum with any model produce high chi-squared values $\left(\chi_{\text {red }}^{2} \gtrsim 1.3\right)$. A close inspection of the continuum at various energies reveals that the residuals are slightly larger relative to the errors than would be expected by $\chi^{2}$ statistics, indicating that we have underestimated the errors. Because of the high signal-to-noise of the Coma observations, the statistical errors no longer completely dominate over channel-to-channel systematic effects, caused by, e.g., differing/variable charge transfer inefficiencies across the detectors and/or between observations, which were obtained over a two year period. We add a $3 \%$ systematic error to the count rate of each channel in the final spectrum to account for these uncertainties, which leads to more reasonable values of $\chi_{\text {red }}^{2} \sim 1$.

\subsubsection{XMM EPIC-pn and Suzaku HXD-PIN Cross-Calibration}

Joint fits between data from different instruments/missions require a careful consideration of the relative overall calibration if the validity of fits are to be believed. Instead of directly finding the cross-normalization through other observations of a spectrally simple source, such as the Crab, we choose to use the XIS0 chip of the Suzaku XRT as an intermediary. An advantage of this method is that it does not require any assumptions about the stability of the absolute calibration of each instrument between 
calibration observations and our observations. To justify our use of the XIS0 data to calibrate the absolute flux level, we compare the flux observed by XIS0 to the ROSAT 0.5-2 keV flux, which was derived using Snowden's ESAS software package (Snowden et al. 1994); these fluxes agree to within $1 \%$.

The overlapping spatial and spectral coverage of the XMM EPIC-pn and XIS0 instruments allows a trivial comparison of the flux for a region on the sky. We extract an XIS0 spectrum from the same region as XMM spectrum R10, and we generate rmf and arf files for this region using the $2-7.5 \mathrm{keV}$ wavelet-smoothed image created from the XMM EPIC-pn data (Schuecker et al. 2004). Though the large XIS PSF $\left(\sim 2^{\prime}\right)$ will scatter photons both into and out of this region to a much greater degree than occurs for $X M M$, this effect is accounted for in the arf and tied to the $X M M$ data. So while spatial inhomogeneities will not impact the comparison, the shapes of the spectra will not necessarily be identical. The overall flux, however, is not sensitive to small variations in the temperature, and so it provides a good quantity to establish the $X M M$-XIS cross-normalization. For this region, we find that the $X M M$ flux is $15 \%$ below the XIS0 flux from 2-7.5 keV, and the XMM flux, extrapolated to 0.5-2 $\mathrm{keV}$, is similarly $15 \%$ below the ROSAT flux, and so we scale the summed $X M M$ arf by this factor.

The cross-normalization between the XIS chips and the HXD-PIN has been well studied for observations of the Crab nebula (Ishida et al., Suzaku Memo 2007-116). We adopt their PIN/XIS0 relative normalization factor of $1.132 \pm 0.014$, increasing the PIN arf, and thus lowering the measured flux, by $13.2 \%$.

The associated systematic error for both cross-normalization corrections is estimated to be $1-2 \%$. However, the normalization of the R10 spectrum may differ from that of the other $X M M$ region spectra, and also the XIS0-PIN relative normalization

\footnotetext{
${ }^{6}$ http://www.astro.isas.ac.jp/suzaku/doc/suzakumemo/suzakumemo-2008-03.pdf
} 
may be different due to the fact that Coma is spatially diffuse while the Crab nebula is comparable in size to the XIS resolution. These issues suggest that the true cross-normalization systematic uncertainty is probably larger. We therefore take the combined cross-normalization systematic error to be $5 \%$, which is about as large as can be reasonably allowed by the simple constraint that a model can be continuously fit across the $12 \mathrm{keV}$ boundary between the $X M M$ and PIN spectra. Specifically, we vary the cross-normalization until the average of the highest signal-to-noise PIN channels, covering $12 \mathrm{keV}<E<16 \mathrm{keV}$, disagrees with the model by $\sim 2-3 \sigma$.

\subsubsection{Cosmic X-ray Background}

We modeled the cosmic X-ray background (CXB) spectrum shape following Boldt (1987), specifically using the analytical form proposed by Gruber et al. (1999) based on the HEAO-1/A2+A4 data. This shape is well-established over the energy range $3<E<60 \mathrm{keV}$ and has been confirmed in subsequent measurements (e.g. Revnivtsev et al. 2003; Churazov et al. 2007; Ajello et al. 2008). We adopt a $10 \%$ larger normalization of the spectrum, relative to the original $H E A O-1$ determination, to agree with the more recent measurements by INTEGRAL (Churazov et al. 2007). This increase is further justified by, and consistent with, the $(8 \pm 3) \%$ higher normalization found with Swift (Ajello et al. 2008). Though these most recent measurements lie slightly, but systematically, above the canonical spectrum, as noted by Ajello et al. (2008) they are not inconsistent with other observations at $E>10 \mathrm{keV}$. At the peak of the CXB spectrum, the measurement precision of HEAO-1 is 10\% (Marshall et al. 1980), and the measurement made with the BeppoSAX PDS is consistent at the $90 \%$ level with a normalization $12 \%$ larger (Frontera et al. 2007). In XSPEC, we model the 
Table 3.2. Fits to Joint $X M M$-PIN spectra

\begin{tabular}{lccccc}
\hline \hline Model & $\begin{array}{c}k T \\
(\mathrm{keV})\end{array}$ & $\begin{array}{c}\text { Norm. } \\
\left(\mathrm{cm}^{-5}\right)\end{array}$ & $\begin{array}{c}\Gamma \text { or } k T \\
(\boldsymbol{\phi}\end{array}$ & Norm. $^{\mathrm{c}}$ & $\chi^{2} /$ dof \\
\hline Single T & $8.45 \pm 0.06$ & $0.218 \pm 0.001$ & - & - & $1676.05 / 1689$ \\
$\mathrm{~T}+\mathrm{IC}^{\mathrm{d}}$ & $8.42 \pm 0.06$ & $0.218 \pm 0.001$ & -1.6 & $(4.6 \pm 3.5) \times 10^{-9}$ & $1671.29 / 1688$ \\
$\mathrm{~T}+\mathrm{IC}^{\mathrm{d}}$ & $8.45 \pm 0.07$ & $0.217 \pm 0.002$ & 2.0 & $\left(2_{-2}^{+12}\right) \times 10^{-4}$ & $1676.18 / 1688$ \\
$2 \mathrm{~T}^{\mathrm{e}}$ & 8.0 & 0.17 & 10.1 & 0.05 & $1672.34 / 1687$ \\
$\mathrm{~T}_{\text {map }}$ & - & - & - & - & $1684.35 / 1690$ \\
\hline
\end{tabular}

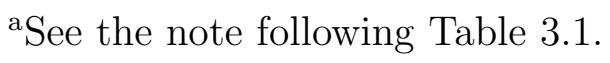

${ }^{\mathrm{b}}$ Value is $\Gamma$ for the $\mathrm{T}+\mathrm{IC}$ model and $k T$ (in $\mathrm{keV}$ ) for the $2 \mathrm{~T}$ model.

${ }^{c}$ Value is the normalization of the power-law component for the $\mathrm{T}+\mathrm{IC}$ model, which is the photon flux at a photon energy of $1 \mathrm{keV}$ in units of photons $\mathrm{cm}^{-2} \mathrm{~s}^{-1} \mathrm{keV}^{-1}$. For the $2 \mathrm{~T}$ model, the value is the normalization of the second APEC thermal model (see the note following Table 3.1) in units of $\mathrm{cm}^{-5}$.

${ }^{d}$ Value of $\Gamma$ is fixed when deriving errors.

eParameters unconstrained. 
CXB as

$$
\begin{aligned}
\mathrm{CXB}(E)= & 1.056 \times 10^{-2}\left(\frac{E}{1 \mathrm{keV}}\right)^{-1.29} e^{-E /(41.13 \mathrm{keV})} \\
& \text { photons } \mathrm{cm}^{-2} \mathrm{~s}^{-1} \mathrm{keV}^{-1}
\end{aligned}
$$

where the normalization is set by a $2^{\circ} \times 2^{\circ}$ solid angle of the sky to match the provided response file (see $\S 3.3 .1$ ).

Cosmic variance due to large scale structure depends on the solid angle of the observation $\left(\Omega=0.32 \operatorname{deg}^{2}\right.$ for the PIN) and on the cut-off flux of removed point sources $\left(S_{\text {cut }}\right)$, determined by the $X M M$ source completeness (Finoguenov et al. $2004)$ to be $S_{\text {cut }}(12-70 \mathrm{keV})=2.2 \times 10^{-13} \mathrm{erg} \mathrm{s}^{-1} \mathrm{~cm}^{-2}$. Since the variance $\sigma_{\mathrm{CXB}} / I_{\mathrm{CXB}} \propto \Omega^{-0.5} S_{\text {cut }}^{0.25}$, we can estimate the variance in our observation relative to another measurement assuming a $\log N-\log S$ relation of $N(S) \propto S^{-1.5}$. Using the HEAO-1 A2 estimate (Shafer 1983; Barcons et al. 2000; Revnivtsev et al. 2003) with $\Omega=15.8 \mathrm{deg}^{2}, S_{\text {cut }}=8 \times 10^{-11} \mathrm{erg} \mathrm{s}^{-1} \mathrm{~cm}^{-2}$, and $\sigma_{C X B} / I_{C X B}=2.8 \%(1 \sigma)$, we find a variance of $7.4 \%$ (90\% confidence), which we take as an additional systematic error in the PIN flux. To account for the $10 \%$ discrepancy between the HEAO-1 and the INTEGRAL (Churazov et al. 2007) and Swift (Ajello et al. 2008) observations, we also estimate the standard deviation of these measurements to be $7 \%$ (90\% confidence). Adding these uncertainties in quadrature, we take the total systematic error in the CXB normalization to be $10 \%$. Below $20 \mathrm{keV}$, the CXB emission is $\lesssim 10 \%$ of the total flux, and it just becomes the dominant source of emission at $\sim 50 \mathrm{keV}$.

For the $X M M$ data, the background spectra include unresolved point sources that make up the CXB, so they do not need to be modeled. 


\subsubsection{Point Sources}

Point sources in the XMM-Newton Coma mosaic have already been identified by Finoguenov et al. (2004), who also give their count rates in three energy bands (0.5-1 $\mathrm{keV}, 1-2 \mathrm{keV}$, and $2-4.5 \mathrm{keV}$ ). For each of the 72 sources, we assume the spectrum to be described by an unabsorbed power law and fit this model to each spectrum. We found that the sum of all these models, weighted by $w^{i}$ according to their positions, could be more concisely described by the sum of two power laws with photon indices 2.1 and 1.6 and normalizations $8.54 \times 10^{-5}$ and $1.23 \times 10^{-4}$ photons $\mathrm{cm}^{-2} \mathrm{~s}^{-1} \mathrm{keV}^{-1}$ at $1 \mathrm{keV}$, respectively. While a simple power law description poorly characterizes some of the sources, care is taken to ensure that individual fits, when extrapolated to high energies, are not unphysical. Their composite spectrum accounts for $\lesssim 1 \%$ of the $X M M$ flux and is therefore unimportant relative to other systematic effects. For this reason we do not go to the extra effort to exclude the sources from the XMM spectra. Assuming the spectral fits are reasonably valid, the point sources account for $\sim 10 \%$ of the CXB at PIN energies. During fits of the joint XMM and Suzaku data, we include this point source composite model for both spectra.

The brightest of these point sources is X Comae, a background AGN with a flux $\sim 10 \times$ brighter than any other source in the field. From XMM RGS observations, it is known to have a steep spectrum $(\Gamma \sim 2.4)$ and to vary in flux by about a factor of 2 over the course of 1 year (Takei et al. 2007). However, due to its position, nearly $90 \%$ of the flux from X Comae is not detected by the HXD, so this source does not significantly contribute to the PIN spectrum. 


\subsection{Spectral Fits}

In our spectral fits, all model components are absorbed by the neutral hydrogen column density toward Coma, $N_{H}=9 \times 10^{19} \mathrm{~cm}^{2}$ [average of values derived from Dickey \& Lockman (1990) and Kalberla et al. (2005)], though this absorption is negligible at energies above $2 \mathrm{keV}$. In general, we characterize the dominant thermal emission in XSPEC with the APEC model for $E<40 \mathrm{keV}$ and with the MeKa model for $E>40 \mathrm{keV}$. In the currect version of XSPEC, the APEC and MeKaL models are undefined above $50 \mathrm{keV}$, though the MeKa and bremsstrahlung models are defined. We tie the parameters of the MeKa model to the APEC parameters, except for the MeKa normalization, which we reduce relative to the APEC normalization by $5.5 \%$ to bring the models into agreement at high energies. Also, the abundances relative to solar and the redshift are fixed, to 0.24 and 0.0232 respectively (see $\S 3.3 .2$ ). This value for the abundance is based on fits to the final weighted and summed $X M M$ spectrum alone, and the best-fit abundances of all the individual spectra from the 10 regions is also consistent with this value. Since we are interested in continuum features, the exact choice for the abundance does not strongly affect the results. The spectral fitting results are summarized in Table 3.2.

\subsubsection{Joint XMM-Newton and Suzaku Spectral Fits Without Considering Systematic Errors}

We simultaneously fit the Suzaku HXD-PIN and XMM-Newton EPIC-pn spectra for the PIN FOV. First, we consider only a single temperature fit, in order to establish whether the addition of a nonthermal component actually improves the fit (Fig. 3.4). We find a best fit temperature of $8.45 \pm 0.06 \mathrm{keV}$, which is in general agreement 
with similar fits to the PIN data $(8.3 \pm 0.3 \mathrm{keV})$ and $X M M$ data $(8.37 \pm 0.12 \mathrm{keV})$ individually. Note that the dip at $15 \mathrm{keV}$ is a known problem with the current NXB model (Mizuno et al., Suzaku Memo 2008-037). Since each spectrum is individually described by the same average temperature, the existence of excess emission at hard energies is unlikely. While all of these temperatures are slightly higher than the cluster-wide average temperature of $8.2 \mathrm{keV}$ (Hughes et al. 1993), the energy range in this and similar fits typically extends to energies below $2 \mathrm{keV}$ and thus includes more low temperature gas.

The addition of a power-law nonthermal component produces a formally better description of the spectra, according to the f-test, improving the overall fit (Table 3.2), but only for a photon index $\Gamma<0$. Allowing the temperature and power law photon index to vary along with each component's normalization, we find $T=8.42 \pm 0.06$ $\mathrm{keV}$ and $\Gamma=-1.6$, though $\Gamma$ is poorly constrained. If we fix $\Gamma$ to this best-fit value, the IC component is significant at the $2.2 \sigma$ level without considering the effect of systematic uncertainties. However, this photon index is completely inconsistent with the spectral index of the radio halo ( $\Gamma \gtrsim 1.8$, Giovannini et al. 1993). While we might expect a flatter spectrum for IC emission, since the hard X-ray photons are emitted by somewhat lower energy electrons than the radio emission, and the radio spectrum flattens at lower frequencies (Thierbach et al. 2003), a rising IC spectrum with energy is completely unexpected and unphysical. The power law fit, in contrast to finding an actual power law signature in the data, is instead compensating for a slight excess at high energies while minimizing its impact on the overall fit at lower energies. Notice that the residuals in Figure 3.4 above $40 \mathrm{keV}$ lie systematically, if not significantly, above the model. This excess at energies above $40 \mathrm{keV}$ can be explained as a $\sim 2 \%$ underestimate of the NXB, as suggested by the Earth-occulted spectrum (see $\S$ 3.3.1).

\footnotetext{
${ }^{7}$ http://www.astro.isas.ac.jp/suzaku/doc/suzakumemo/suzakumemo-2008-03.pdf
} 
Increasing the background level by $2 \%$ for $E>40 \mathrm{keV}$ results in a best-fit power law component very similar to the model used for the $X M M$ point sources, with $\Gamma \sim 1.6$, but it is not significant at even the $1 \sigma$ level. A similar result is found if the photon index is fixed at $\Gamma=2$ and the NXB above $40 \mathrm{keV}$ is not increased; this fit is shown in Figure 3.5. In this case, the fit is not improved by the addition of a power-law component to the model.

Interestingly, a two-temperature model for the ICM yields only a slightly better fit to the data than does the single temperature model (see Fig. 3.6), though the addition of a second temperature component is probably not formally justified. This result is mainly due to the addition of the $3 \%$ systematic error to the $X M M$ spectrum. Without including that error, a two-temperature model produces a clearly improved fit over a single temperature model, indicating that the addition of this error is somewhat obscuring evidence for a multi-temperature continuum. In either case, the two temperatures are not strongly constrained, but they are broadly consistent with the spatial variations in Coma's temperature (see $\S 3.4 .2$ and Fig. 3.7). Therefore, even before systematic errors are considered, the case for the inclusion of a nonthermal component is not strongly motivated.

\subsubsection{Multiple Thermal Components in Coma}

In most previous attempts to measure a nonthermal component in the hard spectrum of Coma, the thermal emission was modeled as a single temperature plasma characteristic of the average global state of the ICM. However, Coma is known to host temperature variations (e.g. Honda et al. 1998). Generally, all clusters exhibit a multi-temperature ICM (Cavagnolo et al. 2008a), and this is especially true of merging clusters like Coma, which tend to host hot regions due to shocks (Markevitch 


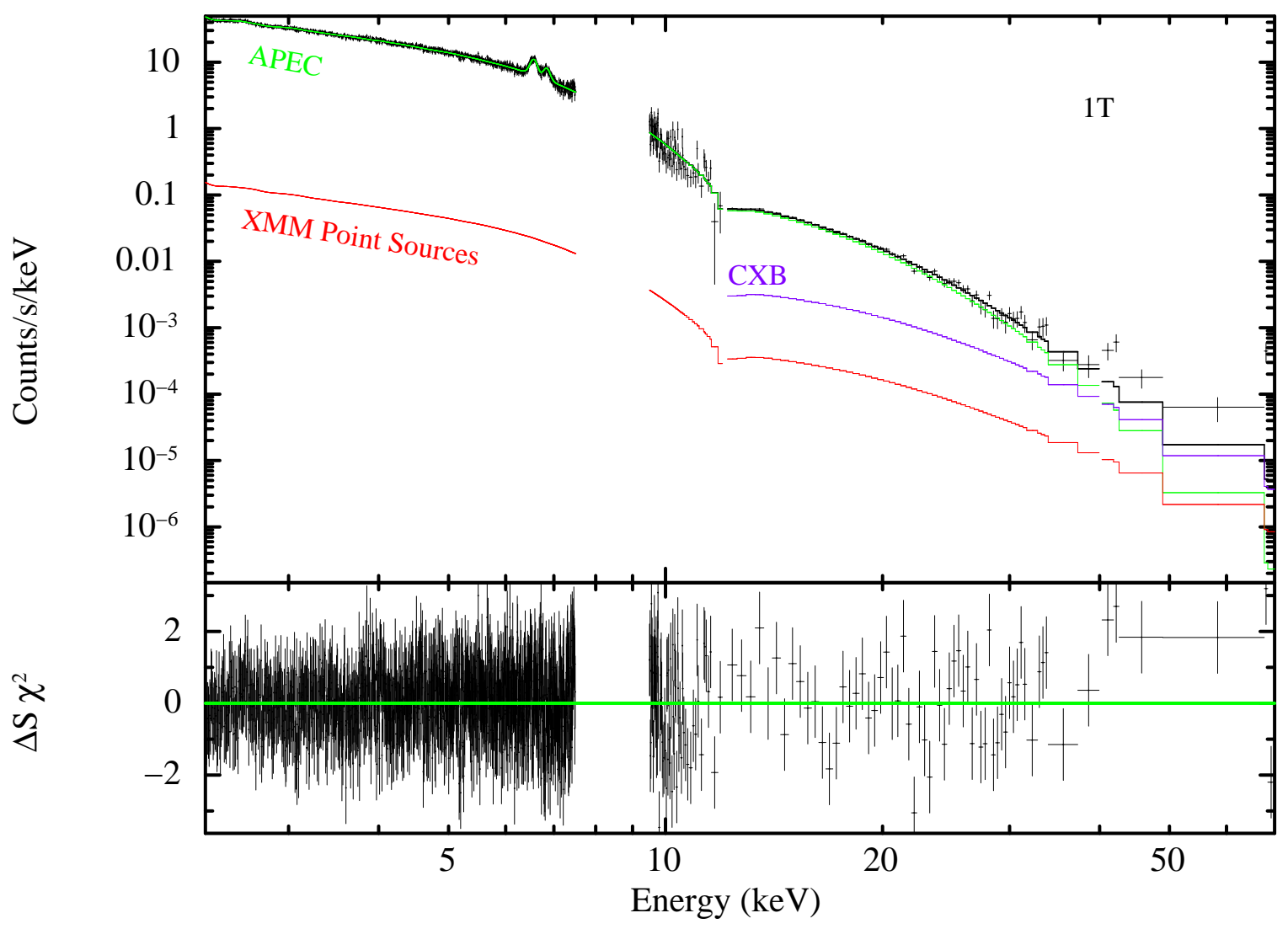

Fig. 3.4. - Suzaku HXD-PIN spectrum $(E>12 \mathrm{keV})$ and the combined XMM spectrum $(E<12 \mathrm{keV})$ corresponding to the spatial sensitivity of the PIN. Shown as solid lines are the best fit models for a single temperature thermal component. The thermal model ("APEC", green) is nearly coincident with the data, though falling below it at higher energies. Also included for all joint fits are the the total spectrum for the "XMM Point Sources" (red) and the Cosmic X-ray Background ("CXB", purple), the latter of which only applies to the PIN spectrum since the CXB is subtracted from the $X M M$ data along with the NXB. 


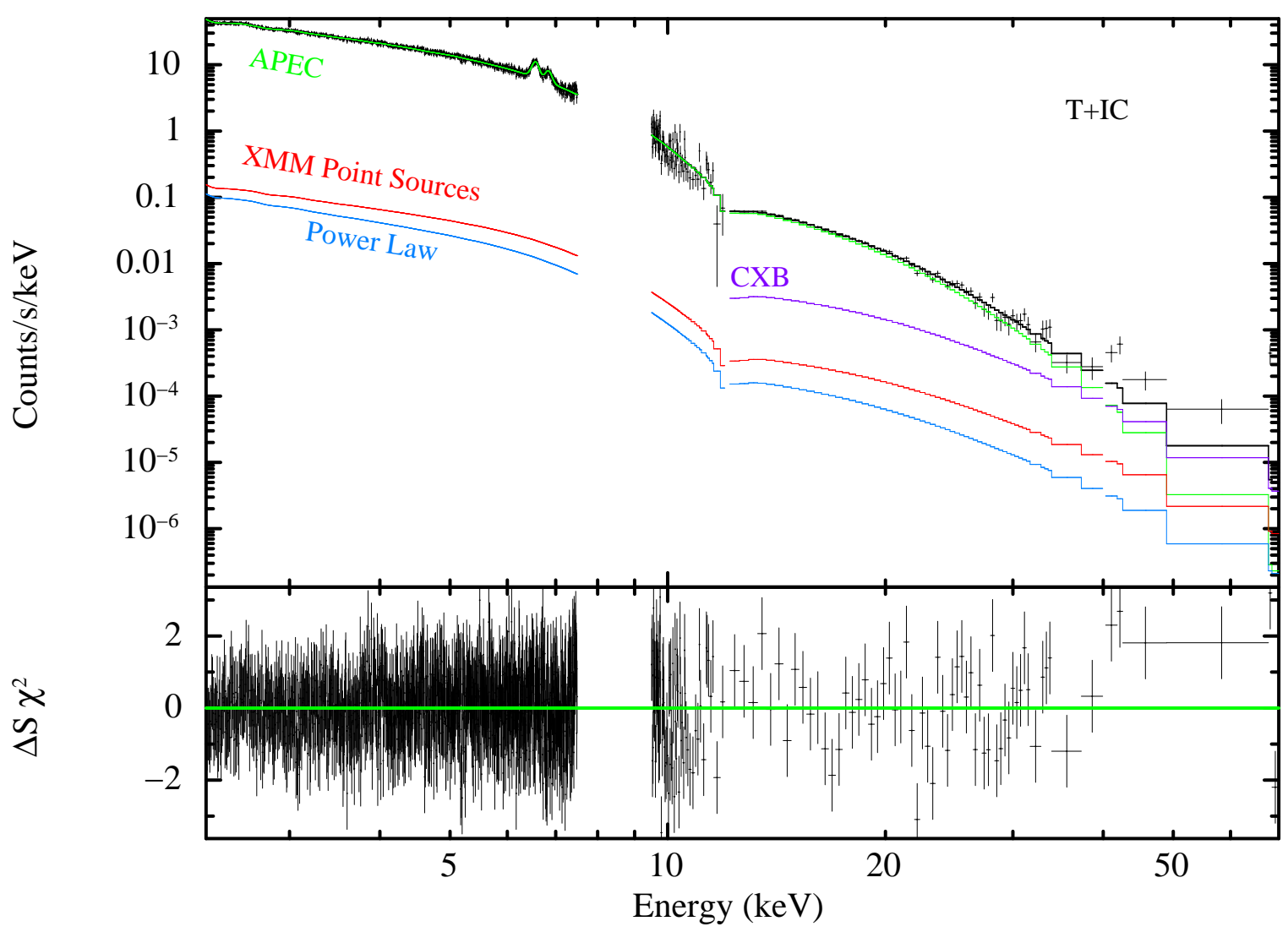

Fig. 3.5.- Suzaku HXD-PIN spectrum $(E>12 \mathrm{keV})$ and the combined XMM spectrum $(E<12 \mathrm{keV})$ corresponding to the spatial sensitivity of the PIN. Shown as solid lines are the best fit models for a single temperature thermal component plus a nonthermal component. The thermal model ("APEC", green) is nearly coincident with the data, though falling below it at higher energies. The nonthermal model ("Power Law", light blue) is the faintest model component for both spectra, and the photon index is fixed at $\Gamma=2.0$. The other two components are described in Figure 3.4. 


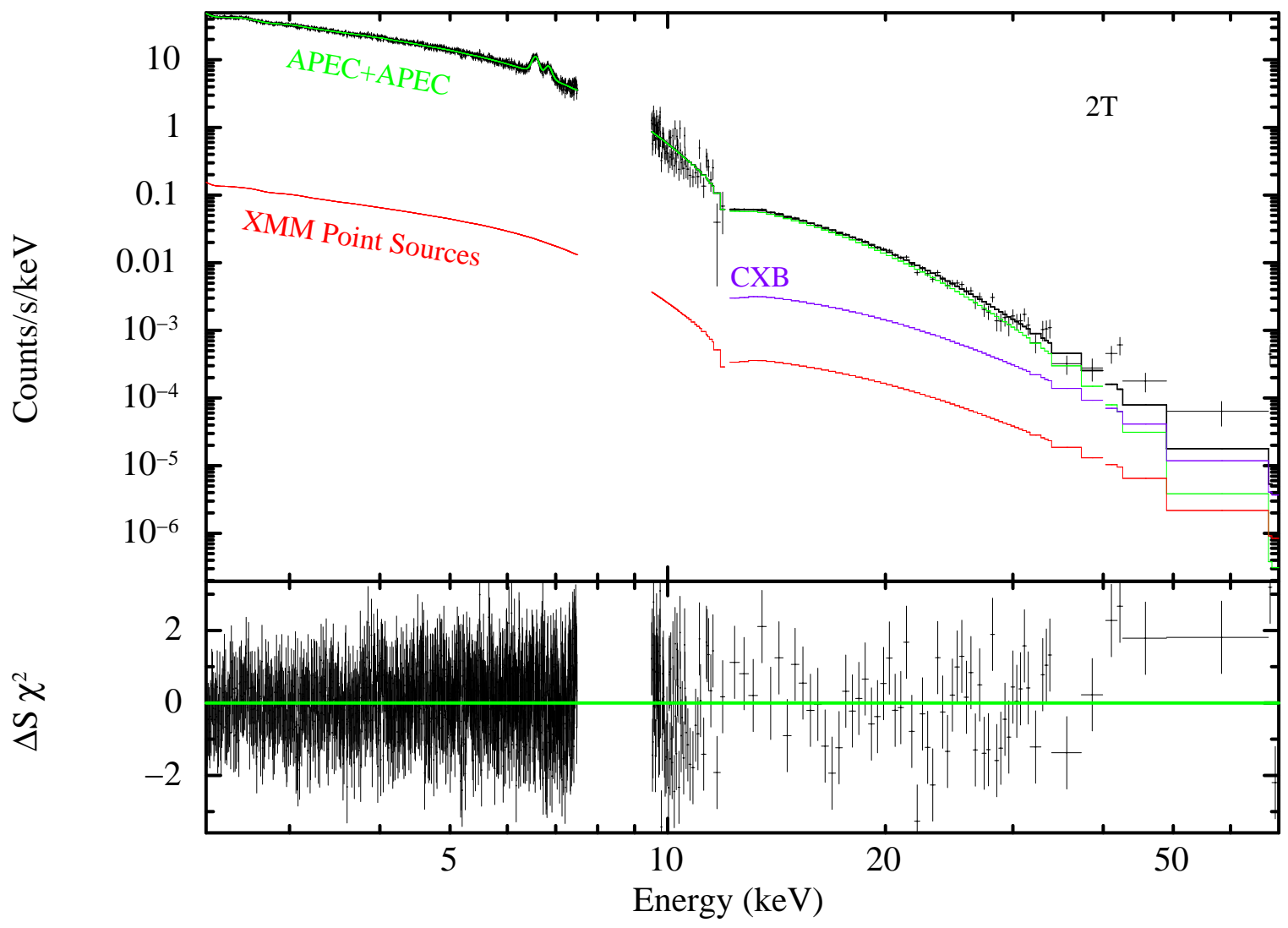

Fig. 3.6. - Suzaku HXD-PIN spectrum $(E>12 \mathrm{keV})$ and the combined XMM spectrum $(E<12 \mathrm{keV})$ corresponding to the spatial sensitivity of the PIN. Shown as solid lines are the best fit models for a two-temperature thermal component. The thermal model ("APEC+APEC", green) is nearly coincident with the data, though falling below it at higher energies. The other two components are described in Figure 3.4. 
et al. 1998). At hard energies, where the exponential turnover in the bremsstrahlung continuum is especially well sampled, even weak higher temperature components can significantly contribute to the flux. Also, these components would lead to a higher average temperature for the ICM than if the average cluster temperature were determined from the spectrum at softer energies, such as from $0.5-10 \mathrm{keV}$.

In the previous section, we found that a two temperature model provided a slightly better description than did a single temperature model of joint fits to the $X M M$ Newton and Suzaku data, especially when ignoring the $3 \%$ systematic error applied to the $X M M$ data. This may indicate that there are multiple temperature components in Coma. The multiple components could occur along the line-of-sight, or in the plane of the sky, or locally (the gas might be multiphase). In fact, previous temperature maps show that Coma certainly has temperature structure which is likely associated with mergers (Watanabe et al. 1999). Here, we test whether this temperature structure alone could reproduce the observed Suzaku PIN spectrum of Coma, without any nonthermal emission. From the XMM-Newton EPIC-pn mosaic of Coma, we constructed a temperature map on a $16 \times 16$ grid with cell size of 4.3 on a side. Each of the spectra were fit with a single temperature APEC model to produce a temperature map that covers the Coma mosaic, as shown in Figure 3.7. We weighted these model fits by the PIN spatial sensitivity and combined them. This resulting model was compared to the PIN spectrum (Table 3.2, row labeled "T map"). Note that only the overall normalization of the $\mathrm{T}_{\text {map }}$ model was allowed to be fit, to compensate for a loss of flux due to incomplete coverage of the map across the HXD FOV. Also, the spectral shape and normalization of each of the thermal models was the same as given by the $X M M-N e w t o n$ temperature, and each model was simply weighted by the average PIN effective area at that position. 


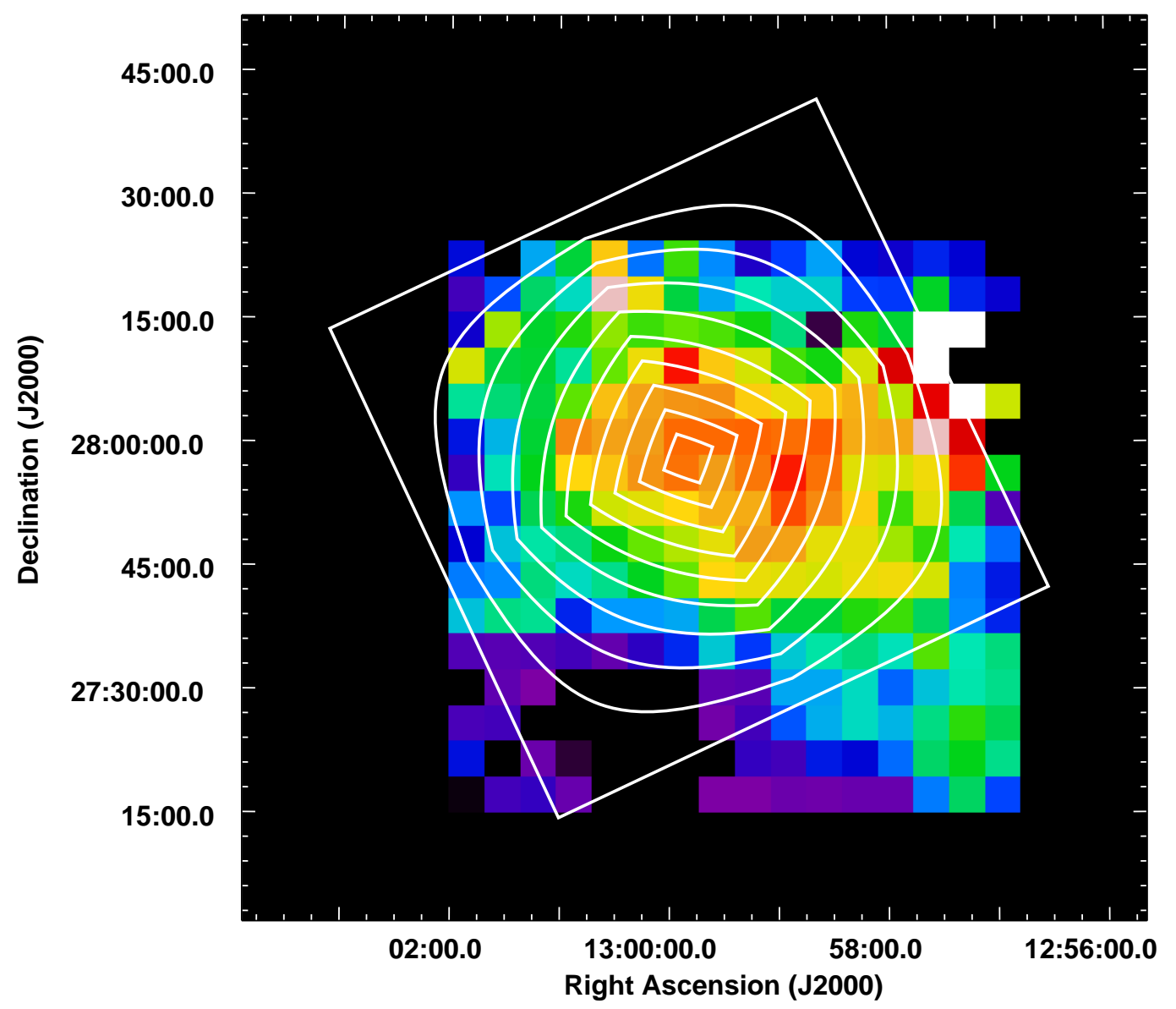

2

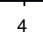

10

12

Fig. 3.7.- XMM-Newton temperature map across Coma with HXD-PIN contours of constant PIN effective area overlaid at $10 \%$ intervals. The XMM-Newton spectra were fit in square spatial regions $4^{\prime} .3$ on a side. The temperatures, given in keV by the color bar, are accurate to either a few tenths of a keV (in the center) or $1-2 \mathrm{keV}$ in lower surface brightness regions. Temperatures shown here were determined from fits to the $0.5-14 \mathrm{keV}$ spectrum in each region. 


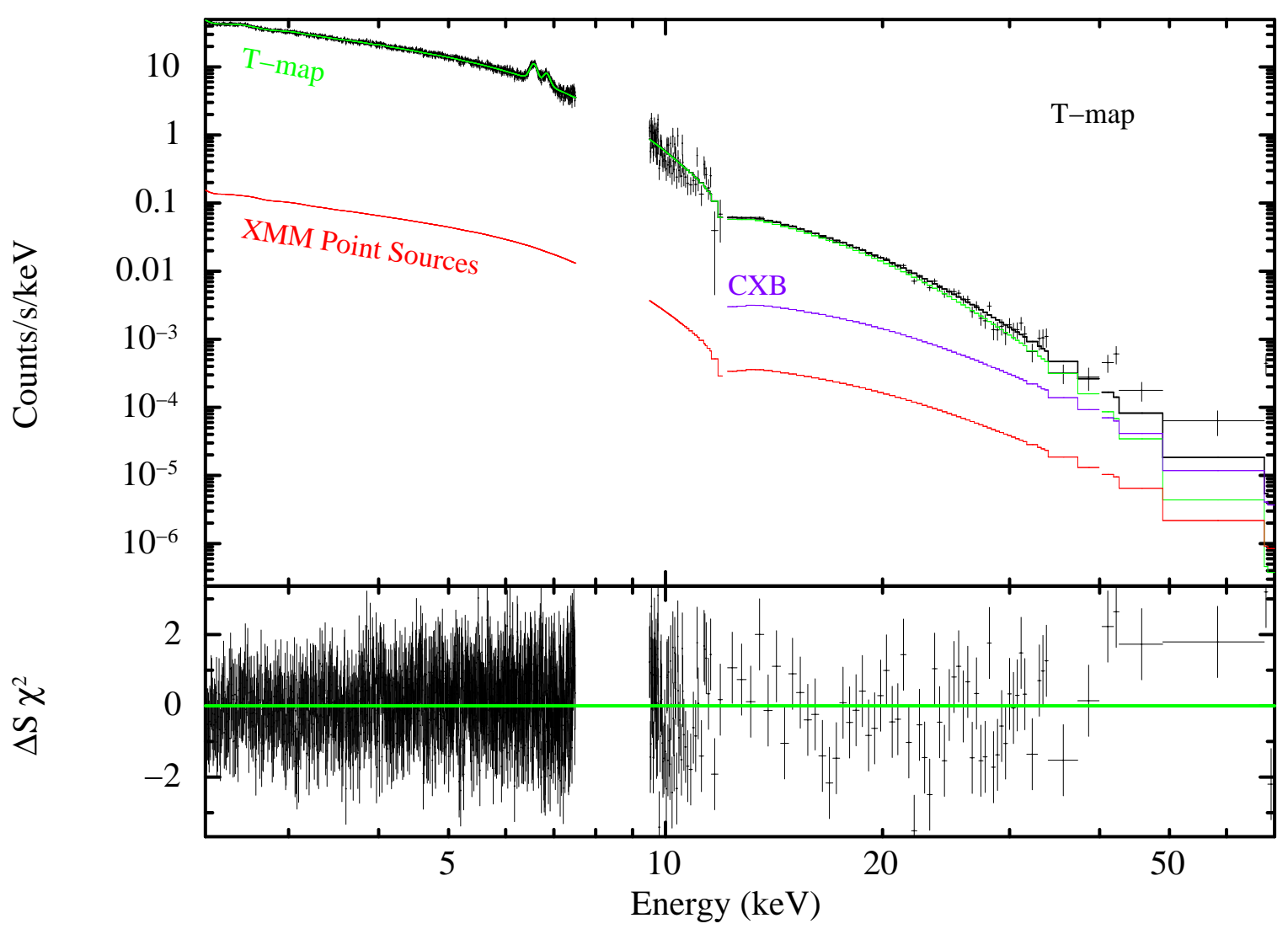

Fig. 3.8. - Suzaku HXD-PIN spectrum $(E>12 \mathrm{keV})$ and the combined $X M M$ spectrum $(E<12 \mathrm{keV})$ corresponding to the spatial sensitivity of the PIN. Shown as solid lines are the combined spectra of the best fit models from the temperature map. The thermal model ("Tmap", green) is nearly coincident with the data, though falling below it at higher energies. The other two components are described in Figure 3.4. 
This $\mathrm{T}_{\text {map }}$ model provides a good fit to the PIN spectrum with no adjustable parameters that affect the spectral shape (Fig. 3.8). While the value of $\chi^{2}$ is worse than for all previous fits, it is only slightly worse, which is not unexpected since it is not really a fit at all. The fact that $X M M$-derived temperatures extrapolated to PIN energies are sufficient to fully account for the PIN spectrum further suggests that no nonthermal hard X-ray excess has been detected with the PIN, especially below $40 \mathrm{keV}$ and probably below $70 \mathrm{keV}$. Also, simulating spectra of similar quality to our data, assuming the $\mathrm{T}_{\text {map }}$ model for the underlying source, yields a joint single temperature fit consistent with that found from the actual data, with $T=8.51 \pm 0.06$ $\mathrm{keV}$.

\subsubsection{Systematic Errors in the Spectrum}

We explicitly consider the systematic error for 3 quantities: the PIN non-X-ray background, the $X M M-S u z a k u$ cross-normalization factor, and the normalization of the CXB as modeled for the PIN spectrum. To test the effect of these systematic errors, we vary the relevant quantity up and down by our estimate of the $90 \%$ systematic error, and evaluate the resulting change in best-fit model parameters. The detection of a nonthermal component cannot be claimed unless it remains robust to variations of these quantities within their systematic errors. Because the largest error is in the normalization of the PIN NXB, we first increase it by $2.3 \%$ from $12-40 \mathrm{keV}$ and $4 \%$ from 40-70 keV and repeat the single temperature plus nonthermal model fit. The new best-fit IC normalization is pushed to zero. Even before considering the effect of other systematic errors, from this exercise alone it is clear that we do not detect nonthermal emission in the HXD-PIN spectrum, given the current uncertainty in the NXB normalization. This fit, with a temperature for the thermal component of 
$8.33 \pm 0.06 \mathrm{keV}$, is shown in Figure 3.9. In fact, the value of $\chi^{2}$ is slightly lower $\left(\chi^{2}=1672.25\right.$ for 1689 dof $)$ than for the nominal PIN NXB single temperature fit. Notice that the residuals above the model for $E>40 \mathrm{keV}$, seen in all the previous spectral fits, have disappeared.

Though we cannot claim to detect nonthermal emission, we can derive an upper limit to its flux based on joint fits to the spectra, including systematic errors in the following way. First, for an assumed photon index which we fix, we find the nominal normalization $N_{\text {nom }}$ and corresponding $90 \%$ upper bound $N_{\text {nom }}^{\text {ul }}$ of the nonthermal component for a single temperature plus power law model, allowing the temperature and normalization of the thermal component to vary. Then, for each systematically uncertain quantity, we set that quantity to the limit bounded by the systematic error in the sense that increases the value of the nonthermal normalization $N_{\mathrm{sys}, i}$, and we fit for it and its $90 \%$ upper bound $N_{\mathrm{sys}, i}^{\mathrm{ul}}$. The statistical and systematic errors of the power law normalization are then given by

$$
\delta_{\text {stat }}=N_{\text {nom }}^{\mathrm{ul}}-N_{\text {nom }}
$$

and

$$
\delta_{\mathrm{sys}, i}=N_{\mathrm{sys}, i}^{\mathrm{ul}}-N_{\mathrm{nom}}^{\mathrm{ul}}=N_{\mathrm{sys}, i}-N_{\mathrm{nom}},
$$

respectively. The final $90 \%$ upper limit is then given by

$$
N_{\mathrm{tot}}^{\mathrm{ul}}=N_{\mathrm{nom}}+\sqrt{\sum_{i} \delta_{\mathrm{sys}, i}^{2}+\delta_{\mathrm{stat}}^{2}} .
$$

We add each systematic error contribution in quadrature because it is unlikely that we chose normalizations for these 3 quantities such that each one disfavors the detection of nonthermal emission in the most severely possible way. 


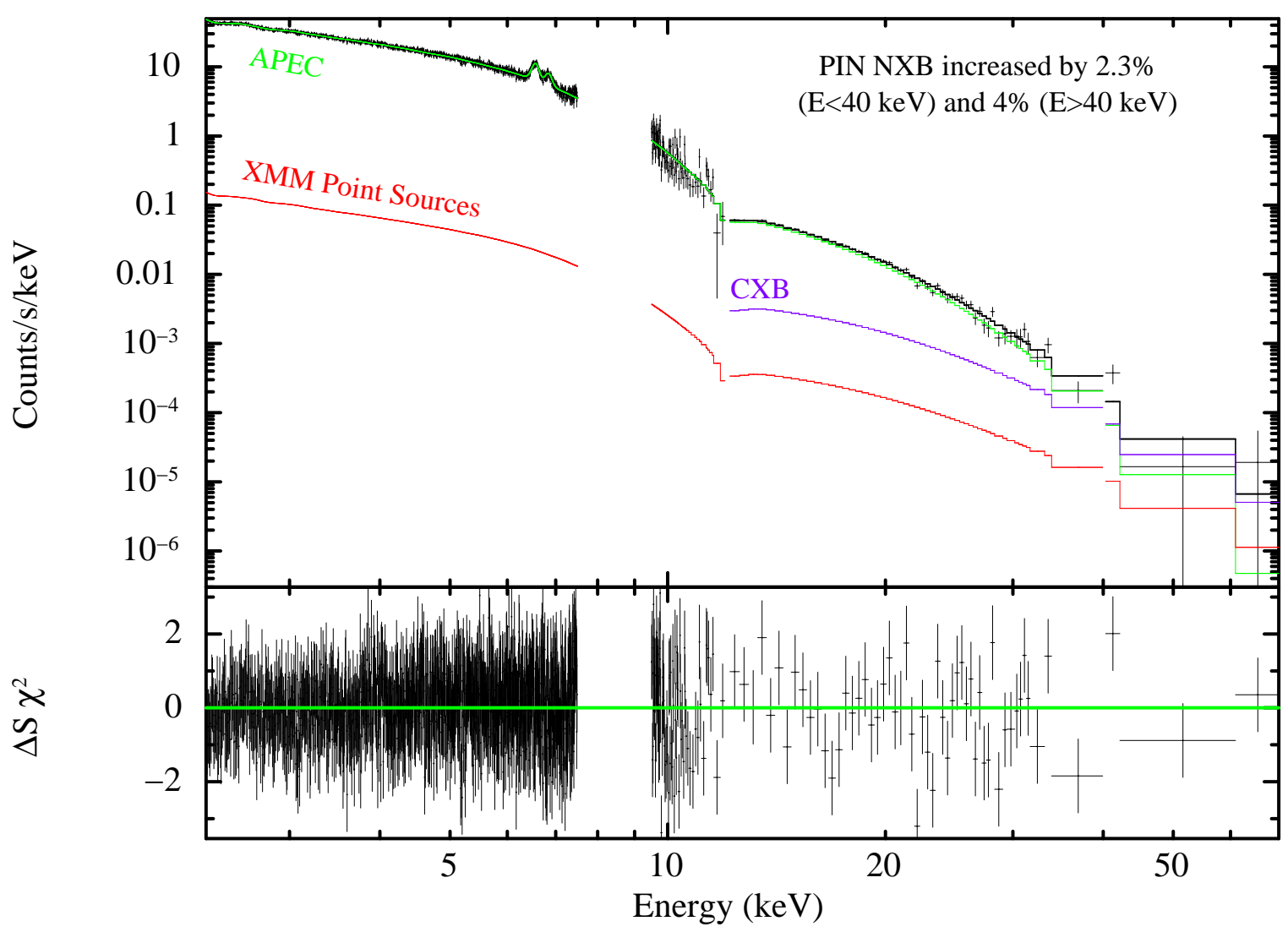

Fig. 3.9. - Suzaku HXD-PIN spectrum $(E>12 \mathrm{keV})$ and the combined $X M M$ spectrum $(E<12 \mathrm{keV})$ corresponding to the spatial sensitivity of the PIN. The PIN NXB is increased to its $90 \%$ confidence limit, which is $2.3 \%$ for $E<40 \mathrm{keV}$ and $4 \%$ for $E>40 \mathrm{keV}$. The thermal model ("APEC", green) is nearly coincident with the data, though falling below it at higher energies. The other two components are described in Figure 3.4. Note that the residuals above the model that exist in the previous fits at $E>40 \mathrm{keV}$ have disappeared. 
The upper limits for a range of assumed photon indices is provided in Table 3.3, and in Figure 3.10 we show, for $\Gamma=2$, the resulting best fit with all 3 systematic quantities set at the limit of their 90\% confidence range. Over the PIN energy band (12-70 keV), the flux is relatively independent of photon index. To compare our results

to the most recent previous detections of nonthermal emission in Coma, we also give the upper limit on the nonthermal flux in the $20-80 \mathrm{keV}$ band, which is $6.0 \times 10^{-12} \mathrm{erg}$ $\mathrm{s}^{-1} \mathrm{~cm}^{-2}$ for $\Gamma=2$. This limit is inconsistent with the $R X T E$ (Rephaeli \& Gruber 2002) and BeppoSAX (Fusco-Femiano et al. 2004) detections by about a factor of 2.5, but at the same level as the upper limit derived by Rossetti \& Molendi (2004) from the BeppoSAX data. If we adopt a $4 \%$ systematic error for the PIN NXB instead of $2.3 \%$ for $E<40 \mathrm{keV}$, which would better agree with that derived from "blank sky" observations, then our upper limit increases by 35\%. Similarly, if we also increase the CXB and XMM-Suzaku cross-normalization to $18 \%$ and 10\%, respectively, our upper limit for $\Gamma=2$ would increase by $50 \%$. In either case, our upper limit still excludes the RXTE and BeppoSAX detections, if FOV differences are ignored (see $\S 3.5$ for a more meaningful comparison).

\subsection{Implications and Discussion}

After modeling all the known possible contributions to the $2-70 \mathrm{keV}$ spectrum, simultaneously fitting for the parameters of thermal and nonthermal spectral components, and taking into account the systematic uncertainty of the PIN NXB, we do not see evidence for IC emission in Coma at our level of sensitivity. We therefore derive an upper limit to nonthermal, hard X-ray emission through a careful consideration of the maximum effect of systematic uncertainties on our ability to detect a nonthermal signal. This conservative upper limit is similar to that derived by Rossetti \& Molendi 


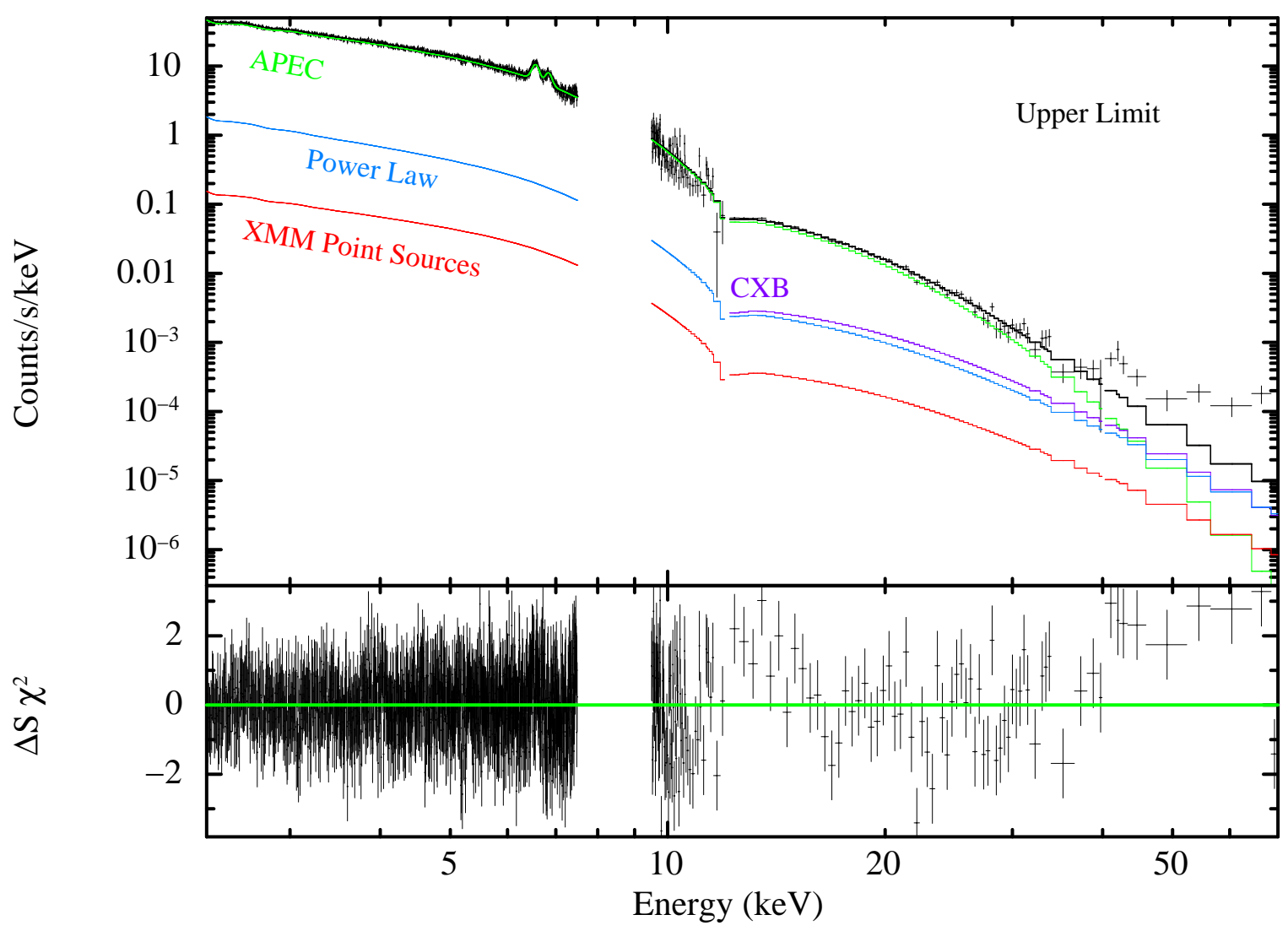

Fig. 3.10.- Suzaku HXD-PIN spectrum $(E>12 \mathrm{keV})$ and the combined XMM spectrum $(E<12 \mathrm{keV})$ corresponding to the spatial sensitivity of the PIN. All quantities with systematic uncertainties (PIN CXB and NXB, the XMM-Suzaku cross calibration) are set to their $90 \%$ confidence limit in the direction that favors the addition of a power law model component to describe the data. The thermal model ("APEC", green) is nearly coincident with the data, though falling below it at higher energies. The nonthermal model ("Power Law", light blue) is shown for $\Gamma=2$ at its $90 \%$ confidence upper limit value. The other two components are described in Figure 3.4. 
Table 3.3. 90\% Upper Limits on IC Flux

\begin{tabular}{|c|c|c|c|c|}
\hline$\Gamma$ & $\begin{array}{c}\text { Norm. }^{\mathrm{a}} \\
\left(10^{-3} \text { photons } \mathrm{keV}^{-1} \mathrm{~cm}^{-2} \mathrm{~s}^{-1}\right)\end{array}$ & $\begin{array}{c}\text { Flux }(12-70 \mathrm{keV}) \\
\left(10^{-12} \operatorname{erg~s}^{-1} \mathrm{~cm}^{-2}\right)\end{array}$ & $\begin{array}{c}\text { Flux }(20-80 \mathrm{keV}) \\
\left(10^{-12} \operatorname{erg~s}^{-1} \mathrm{~cm}^{-2}\right)\end{array}$ & $\begin{array}{l}\text { BeppoSAX Detection } \\
\\
\left(10^{-12} \mathrm{erg} \mathrm{s}^{-1} \mathrm{~cm}^{-2}\right)\end{array}$ \\
\hline 1.0 & 0.155 & 14.4 & 14.9 & \\
\hline 1.1 & 0.220 & 14.2 & 14.4 & \\
\hline 1.2 & 0.311 & 14.1 & 13.9 & \\
\hline 1.3 & 0.439 & 13.9 & 13.4 & \\
\hline 1.4 & 0.617 & 13.8 & 12.9 & \\
\hline 1.5 & 0.860 & 13.5 & 12.3 & \\
\hline 1.6 & 1.18 & 13.1 & 11.6 & \\
\hline 1.7 & 1.58 & 12.4 & 10.7 & \\
\hline 1.8 & 2.04 & 11.4 & 9.51 & \\
\hline 1.9 & 2.48 & 9.83 & 7.98 & \\
\hline 2.0 & 2.70 & 7.64 & 6.01 & $15 \pm 5$ \\
\hline 2.1 & 2.70 & 5.46 & 4.15 & \\
\hline 2.2 & 2.56 & 3.71 & 2.73 & \\
\hline 2.3 & 2.36 & 2.46 & 1.74 & \\
\hline 2.4 & 2.15 & 1.62 & 1.11 & \\
\hline
\end{tabular}

${ }^{a}$ Normalization of the power-law component for the $\mathrm{T}+\mathrm{IC}$ model, which is the photon flux at a photon energy of $1 \mathrm{keV}$.

${ }^{\mathrm{b}}$ Flux $(20-80 \mathrm{keV})$, as reported in Fusco-Femiano et al. (2004). 
(2004) and is inconsistent with claimed detections using RXTE (Rephaeli \& Gruber 2002) and BeppoSAX (Fusco-Femiano et al. 2004) by approximately a factor of 2.5 . However, it should be noted that we do not include potentially lost emission due to PIN vignetting from any of our flux upper limits relative to the larger FOVs of $R X T E$ and BeppoSAX, which have collimator FWHM of $1^{\circ}$ and $1.3^{\circ}$, respectively.

If IC emission follows the radio synchrotron emission [as derived from the point source-subtracted radio image from Deiss et al. (1997)], as it would for a uniform $B$ field throughout the cluster, our upper limits imply a total IC flux $1.7-2 \times$ larger would be found inside an RXTE/BeppoSAX-like FOV. We also consider a more detailed spatial distribution for the underlying IC emission, derived from the re-acceleration model of Brunetti \& Blasi (2005), in which relativistic protons collide with electrons in the ICM that are then re-accelerated by Alfvén waves due to cluster mergers. Given the radio spectrum of Coma, this model predicts that the smaller FOV of the Suzaku HXD-PIN would lead to an underestimate of the nonthermal flux by a factor of $2-2.5$ (possibly 3 under extreme circumstances). If this model for the spatial distribution of the nonthermal emission is correct, then our upper limit is just consistent with the measurements of Rephaeli \& Gruber (2002) and FuscoFemiano et al. (2004). (However, the Brunetti \& Blasi (2005) model actually predicts a nonthermal flux considerably below the BeppoSAX and RXTE detections.) Because any spatial variation of the magnetic field strength is unknown, a direct comparison between these missions is not possible with any precision. Under the reasonable assumption that $B$ decreases with radius, our upper limit will be $\gtrsim 2 \times$ larger, so our result cannot definitively rule out the detections discussed above. Regardless of this issue, the upper limit is approximately the same as or slightly higher than the upper limit range found by Rossetti \& Molendi (2004). 
However, the BeppoSAXPDS measures a $20-80 \mathrm{keV}$ flux for the Crab of $1.23 \times 10^{-8}$ $\mathrm{erg} \mathrm{s}^{-1} \mathrm{~cm}^{-2}$ (Kirsch et al. 2005), while the Suzaku PIN flux over this energy range is $1.56 \times 10^{-8} \mathrm{erg} \mathrm{s}^{-1} \mathrm{~cm}^{-2}$, after applying the $13.2 \%$ correction to bring the PIN spectrum into agreement with the XIS fluxes (Ishida et al., Suzaku Memo 2007-118). This $21 \%$ flux difference implies our upper limit would be $4.7 \times 10^{-12} \mathrm{erg} \mathrm{s}^{-1} \mathrm{~cm}^{-2}$ on the BeppoSAX scale, which is on the lower end of the range estimated by Rossetti \& Molendi (2004). Also, even if we only detect one-third of the total emission observed by the BeppoSAX PDS, we would just barely exclude the nominal value of the FuscoFemiano et al. (2004) measurement.

Assuming the differing measurements of nonthermal emission are not due to the IC radiation having a larger extent, what might be the cause of this discrepancy? While it could be explained by a greater point source contamination at hard energies for the $R X T E$ and BeppoSAX missions due to their larger FOVs, most likely we differ in our results because of different considerations of the thermal gas. For both detections, the gas temperature was found to be lower than our nominal value of $8.45 \mathrm{keV}$. Fixing the gas temperature to their assumed values in our fits yields a $\Gamma=2.0$ nonthermal component significance $>4 \sigma$, without including systematic effects, for $T=7.67 \mathrm{keV}$ $(R X T E)$ and $T=8.2 \mathrm{keV}($ BeppoSAX); however, these fits are poor relative to fits in which the temperature is a free parameter. Though these temperatures differ from our best-fit value by only a few percent, the exponential decline of bremsstrahlung continuum at high energies amplifies even small differences. The lower measurements of the ICM temperature appear not to be due to the inclusion of data at low energies $(E<1 \mathrm{keV})$, which can bias average temperature estimates low. Most likely, the larger FOVs of $R X T E$ and BeppoSAX allowed the inclusion of emission from more cool gas in the cluster outskirts than was observed by Suzaku. This emission would

\footnotetext{
${ }^{8}$ http://www.astro.isas.ac.jp/suzaku/doc/suzakumemo/suzakumemo-2007-11.pdf
} 
serve to lower the average observed temperature, which is primarily determined from emission at lower energies $(E<10 \mathrm{keV})$. But, as evidenced by the temperature map in Figure 3.7, a distribution of higher-than-average temperature regions can effectively increase the average gas temperature observed at high energies, as first seen by Neumann et al. (2003).

We take the reasonably good agreement between the thermal models derived from the XMM temperature map and the PIN spectrum to mean that we essentially only detect thermal emission from Coma out to $70 \mathrm{keV}$. This result is fully consistent with recent INTEGRAL detections of extended hard X-ray emission. Renaud et al. (2006a) performed a point-by-point spectral comparison between $X M M$-derived and INTEGRAL-derived temperatures and found that they followed a strict one-to-one correlation. Similarly, Eckert et al. (2007a) characterized a surface brightness excess relative to the $X M M$ data, which they found to be best described by extended hot, thermal emission at a $T \sim 12 \pm 2 \mathrm{keV}$. This excess coincides with the hotter temperatures $(T \sim 10-11 \mathrm{keV})$ to the west of the PIN pointing center in Figure 3.7.

From our upper limit on the flux of IC emission, we can derive a lower limit on the average magnetic field strength $B$ as shown by Harris \& Romanishin (1974). Equation (3.2) refers to the total energy emitted for one electron; it is more useful to consider the ratio of monochromatic fluxes $F_{R}\left(\nu_{R}\right), F_{X}\left(\nu_{X}\right)$ at frequencies $\nu_{R}, \nu_{X}$, for a power law distribution of electrons, from which we can derive an expression for the magnetic field as

$$
B=C(p)(1+z)^{(p+5) /(p+1)}\left(\frac{F_{R}}{F_{X}}\right)^{2 /(p+1)}\left(\frac{\nu_{R}}{\nu_{X}}\right)^{(p-1) /(p+1)}
$$

where $p$ is the index of the electron distribution $N(E) \propto E^{-p}$ and is related to the spectral index $\alpha\left(F_{\nu} \propto \nu^{-\alpha}\right)$ by $p=2 \alpha+1$. The value of the proportionality constant 
$C(p)$ can be found from the ratio of the synchrotron flux (Longair 1994, eqn. 18.49) to the IC flux (Rybicki \& Lightman 1979, eqn. 7.31). Assuming that the electron energy distribution does not turn over significantly at low energies and that $\alpha=1$, we find $B>0.15 \mu \mathrm{G}$. This limit is still below the equipartition value of $0.5 \mu \mathrm{G}$ (Giovannini et al. 1993), and it is well below the line-of-sight estimates of a few $\mu \mathrm{G}$ derived from Faraday rotation measure (RM) studies (Feretti et al. 1995). Note that the Faraday RM magnetic field estimates are sensitive to the $B$ field geometry and may imply a field strength larger than the volume-averaged value if $B$ is preferentially aligned along filamentary structures on small scales (Petrosian 2001). Also, we are unable to put interesting constraints on the relativistic energy budget of Coma, since our lower limit includes the equipartition estimate of $B$, which defines the minimum energy in relativistic components and would not imply a significant contribution to the energy budget of Coma.

It has been noted that the hard excess detected by Eckert et al. (2007a) also corresponds to the peak in the point source-subtracted image from Deiss et al. (1997), potentially indicating that the hard emission could in fact be nonthermal in origin (Eckert et al. 2007b). In fact, we suspect that this peak, which appears tantalizingly like a small radio relic, is not a true feature of the halo, but instead is the result of imperfect source subtraction. Due to the large beam size used to create the diffuse radio image, extended emission from radio galaxies might not have been properly subtracted using a point source list. We point out that the strongest radio source $(1256+282$ or 5C 4.81, centered on NGC 4869) in Coma is near this position, is a head-tail radio galaxy with a steeper spectral index than rest of the halo (Giovannini et al. 1993), and that its tail extends to the west (O'Dea \& Owen 1985, see Fig. 1(j)) and turns north (Venturi et al. 1990, see Fig. 2). Subtracting a point source from this 
morphology would leave a residual very similar to that in the Deiss image. Therefore, any relation between the location of hard emission and this radio feature probably should be regarded as coincidence.

As the calibration of the NXB model improves, constraints on IC emission in Coma will tighten, possibly leading to a detection. The uncertainty in the current background model, "bgd_d," is more than a factor of 2 lower than the original model. However, the existence of nonthermal emission in the Coma cluster may have to be determined by future missions - particularly, those missions with hard X-ray imaging capabilities like NuStar ${ }^{9}$, Astro-H (previously NeXT) ${ }^{10}$, and Simbol-X ${ }^{11}$. If the IC emission is localized, then our joint fitting-technique can be used for many much smaller regions where temperature-mixing will be less significant and the IC component will be relatively stronger. Also, the $B$ field strength can be derived spatially across a cluster, yielding a better estimate of the possible pressure support provided by relativistic components in the ICM, which could modify mass estimates that depend on the hydrostatic equilibrium state of the cluster gas.

\footnotetext{
${ }^{9}$ http://www.nustar.caltech.edu/

${ }^{10} \mathrm{http}: / /$ www.astro.isas.ac.jp/future/NeXT/

${ }^{11}$ http://www.asdc.asi.it/simbol-x/
} 


\section{Chapter 4}

\section{The Lack of Diffuse, Non-thermal}

\section{Hard X-ray Emission in the Coma}

\section{Cluster: The Swift BAT's Eye}

\section{View $^{1}$}

\section{Abstract}

The Coma cluster of galaxies hosts the brightest radio halo known and has therefore been the target of numerous searches for associated inverse Compton (IC) emission, particularly at hard X-ray energies where the IC signal must eventually dominate over thermal emission. The most recent search with the Suzaku Hard X-ray Detector (HXD) (Wik et al. 2009) failed to confirm previous IC detections (Rephaeli \& Gruber 2002; Fusco-Femiano et al. 2004), instead setting an upper limit 2.5 times below their nonthermal flux. However, due to the relatively smaller field of view of the HXD, this

\footnotetext{
${ }^{1}$ Ready for submission to the Astrophysical Journal
} 
discrepancy can be resolved if the IC emission is very extended, beyond the scale of the cluster radio halo. Using reconstructed sky images from the 58-month Swift BAT all sky survey, the feasibility of such a solution is investigated. Building on Renaud et al. (2006b), we test and implement a method for extracting the fluxes of extended sources, assuming specified spatial distributions. Thermal and nonthermal spatial distributions appropriate for the Coma cluster are simultaneously fit to the 8 energy bands that make up the BAT survey data; the spatial distribution of the detected emission is found to be consistent with a purely thermal origin. The resulting spectra are then jointly fit with an XMM-Newton EPIC-pn spectrum derived from mosaic observations. We find no evidence for large-scale IC emission at a level sufficient to reconcile the Suzaku upper limit with the previous detections. For all nonthermal spatial distributions considered, which span the gamut of physically reasonable IC models, we determine upper limits for which the largest (most conservative) limit is $\lesssim 4.2 \times 10^{-12} \mathrm{erg} \mathrm{s}^{-1} \mathrm{~cm}^{-2}(20-80 \mathrm{keV})$, which corresponds to a lower limit on the magnetic field $B>0.2 \mu \mathrm{G}$. A nominal flux upper limit of $<2.7 \times 10^{-12} \mathrm{erg} \mathrm{s}^{-1} \mathrm{~cm}^{-2}$, with corresponding $B>0.25 \mu \mathrm{G}$, is derived for the most probable IC distribution given the size of the radio halo and likely magnetic field radial profile.

\subsection{Introduction}

The X-ray emission from clusters of galaxies is primarily thermal in origin and is produced by a diffuse population of intergalactic electrons in the ionized intracluster medium (ICM). These electrons coexist with a nonthermal, relativistic electron population in at least some clusters - inferred from observations in the radio regime - which should also radiate at X-ray energies. While thermal emission clearly dominates in the kilo-electron volt (keV) energy range, it declines rapidly outside this range, allow- 
ing the detection of a nonthermal spectral signature as soft or hard excess emission. This possibility is especially promising at hard $(>10 \mathrm{keV})$ energies, where the exponential decline of the thermal bremsstrahlung continuum is distinctly steeper than the expected nonthermal spectrum. Measurements of nonthermal X-ray emission are critical to the determination of the total amount of relativistic constituents in the ICM, which is currently poorly constrained. While no more than $\sim 10 \%$ of the energy in the ICM is tied up in nonthermal components, amounts at or near this level will affect the dynamics and structure of the thermal gas (e.g., Vanderlinde et al. 2010). Specifically, studies that attempt to infer the total masses of clusters from the hydrostatic state of the thermal gas will produce biased mass estimates if the pressure support of relativistic particles and fields is not accurately included. Such mass estimates are the cornerstone from which cluster mass functions are built, which can be used to constrain cosmological parameters; these studies are already underway using observables derived in both the X-ray (e.g., Mantz et al. 2008; Vikhlinin et al. 2009) and microwave (e.g., Vazza et al. 2009, through the Sunyaev-Zel'dovich effect) regimes.

A measurement of the total energy in relativistic ICM components is possible when $\mathrm{X}$-ray and radio nonthermal fluxes are combined. Diffuse, cluster-wide synchrotron radio emission, called radio halos or relics depending on their morphology, imply that both magnetic fields and relativistic electron populations are present on large scales. The total luminosity of a synchrotron-emitting electron is given by

$$
L_{R}=\frac{4}{3} \sigma_{T} c \gamma^{2} \epsilon_{B},
$$

where $\sigma_{T}$ is the Thomson cross-section, $c$ is the speed of light, $\gamma$ is the Lorentz factor of the electron, and $\epsilon_{B}=B^{2} / 8 \pi$ is the energy density of the magnetic field. For 
a collection of relativistic electrons, the value of $L_{R}$ depends both on the number of electrons and on $B$ and cannot independently determine either. However, these same electrons will up-scatter cosmic microwave background (CMB) photons through inverse Compton (IC) interactions, which have a luminosity $L_{X}$ equivalent in form to equation (4.1) but with $\epsilon_{B}$ replaced by the energy density of the CMB. Since both luminosities are proportional to the number of electrons, their ratio gives the volume-averaged magnetic field,

$$
\frac{L_{R}}{L_{X}}=\frac{B^{2} / 8 \pi}{a T_{C M B}^{4}},
$$

where $a$ is the radiation constant and $T_{C M B}$ is the temperature of the CMB. The IC radiation should be observable at hard X-ray energies (Rephaeli 1977). Thus far, IC emission has only been detected at low significance (Nevalainen et al. 2004; Million \& Allen 2009) or, in one case, in a cluster with uncertain radio emission (Eckert et al. 2008; but see also Ajello et al. 2009 and Fujita et al. 2008). The measurement of an IC flux from a synchrotron source directly leads to a simultaneous determination of the average value of $B$ and the relativistic electron density (Harris \& Romanishin 1974; Sarazin 1988). Therefore searches for IC emission coincident with a radio halo or relic are an excellent way to constrain the contribution of relativistic materials in clusters.

The first, and brightest, radio halo was discovered by Willson (1970) in the Coma cluster, and its radio properties have perhaps been the best studied (e.g. Giovannini et al. 1993; Deiss et al. 1997; Thierbach et al. 2003). Coma has been observed by all the major observatories with hard X-ray capabilities (Henriksen \& Mushotzky 1986; Bazzano et al. 1990; Hughes et al. 1993; Rephaeli et al. 1994), and more recently nonthermal detections have been claimed by Rephaeli \& Gruber (2002) with RXTE and 
by Fusco-Femiano et al. $(1999,2004)$ with BeppoSAX, though the latter detection is controversial (Rossetti \& Molendi 2004; Fusco-Femiano et al. 2007). Due to the large field of view (FOV) of these non-imaging instruments and the simple characterization of the thermal gas, the source of this emission remains uncertain. Even more recently, long ( 1 Msec) observations with INTEGRAL have imaged extended diffuse hard X-ray emission from Coma, though it was found to be completely consistent with thermal emission (Renaud et al. 2006a; Eckert et al. 2007a; Lutovinov et al. 2008).

Most recently, Wik et al. (2009) performed a joint analysis of spectra from the XMM-Newton EPIC-pn and Suzaku HXD-PIN instruments - the most sensitive instruments at soft and hard energies to date - of the Coma cluster and were unable to detect IC emission. Instead, they found an upper limit 2.5 times below the detections of Rephaeli \& Gruber (2002) and Fusco-Femiano et al. (2004). However, the narrower FOV of the HXD relative to the collimators of the RXTE PCA/HEXTE and Beppo-SAX PDS leaves open the possibility that the spatial distribution of IC photons is highly extended, and therefore much of the flux was missed by the HXD. The IC would have to be much broader than the size of the radio halo. A uniform IC surface brightness of at least $30^{\prime}$ in radius from the cluster center is sufficient to reconcile these results. Therefore, an imaging analysis at hard X-rays is required to confirm this picture; unfortunately, no focussing hard $(>10 \mathrm{keV}) \mathrm{X}$-ray telescope has yet been deployed. In the meantime, it is possible to perform a crude imaging analysis with coded mask instruments, as previously discussed by Renaud et al. (2006b).

In this work, we report on the spatial and spectral hard X-ray emission from the Coma cluster using the 58-month accumulation of the Swift Burst Alert Telescope (BAT) all-sky survey. From the first 9 months of the survey, Coma is clearly extended (Ajello et al. 2009), and so an accurate measurement of its flux must account for this 
fact. Using models for the spatial distribution of thermal and potential nonthermal emission, we measure the total, extended flux in the 8 energy bands that make up the survey. These fluxes are then converted into spectra, which we jointly fit with an XMM-Newton EPIC-pn spectrum from a spatially identical region. In this way, despite poor spatial resolution $\left(\sim 20^{\prime}\right)$, we are sensitive to any large-scale, extended emission above the detection threshold for the survey. While the sensitivity of the BAT detector is lower than instruments such as the Suzaku HXD-PIN, the survey's large exposure time - thanks to a FOV that sees $1 / 8^{\text {th }}$ of the sky in a single pointing - gives it a comparable, if not superior, overall sensitivity. In Section 4.2, we describe the Swift BAT survey in general and the XMM-Newton EPIC-pn and BAT observations of the Coma cluster specifically. The extraction of spatially extended fluxes from models, along with the specific models themselves, is discussed in Section 4.3. Spectra constructed from these spatial fits are presented in Section 4.4, along with the results of joint fits with the XMM-Newton spectrum. In Section 4.5, we provide upper limits on spatially extended, nonthermal emission, and in Section 4.6 we discuss the implications of our non-detection for the relativistic phase of the ICM of the Coma cluster. In the appendices, we describe the calibration of the survey such that joint fits with $X M M$-Newton are straightforward, and we demonstrate that the BAT instrument intrinsically detects extended emission on the scales of interest here, though with higher uncertainty than for a point source. We assume a flat cosmology with $\Omega_{M}=0.23$ and $H_{0}=72 \mathrm{~km} / \mathrm{s} / \mathrm{Mpc}$ and a luminosity distance to Coma of 98.4 Mpc. Unless otherwise stated, all uncertainties are given at the $90 \%$ confidence level. 


\subsection{Observations}

To achieve the necessary spatial coverage and spectral sensitivity, we take advantage of mosaics of the Coma cluster constructed from observations by the XMM-Newton and Swift satellites. The high sensitivity and good spectral and spatial resolution of the XMM-Newton EPIC-pn data act as a check on the interpretation of the Swift BAT data, allowing the thermal and potentially nonthermal emission at hard energies to be accurately decoupled.

\subsubsection{XMM-Newton EPIC-pn Mosaic Observations}

The observations and processed $X M M$-Newton data used herein are identical to that presented in Wik et al. (2009), where a more detailed description can be found. The $X M M-N e w t o n$ EPIC-pn mosaic of Coma consists of 14 individual pointings, the first set (11 pointings) of which were discussed in Briel et al. (2001). The full 14 pointings considered here were first presented in Schuecker et al. (2004). For joint fitting with Swift BAT spectra, we extract events from a $65^{\prime} .5 \times 65^{\prime} .5$ box centered on the radio halo, which was originally chosen to match the Suzaku Hard X-ray Detector field of view; the region is shown as the outermost contour in Figure 3 of Wik et al. (2009), and also as the box in Figure 4.1 in the present paper. The only modification of our analysis procedure compared to that in Wik et al. (2009) is that the XMM-Newton spectra were not weighted by the spatial response of the Suzaku HXD, since they are not being fit simultaneously with that instrument. No similar weighting is needed to comparison to the Swift BAT data since the BAT survey covers the entire sky and the vignetting of individual pointings is corrected for during processing.

We also use the temperature map derived from the XMM-Newton mosaic and described in Wik et al. (2009) to model the spatial distribution of hard X-ray emission, 

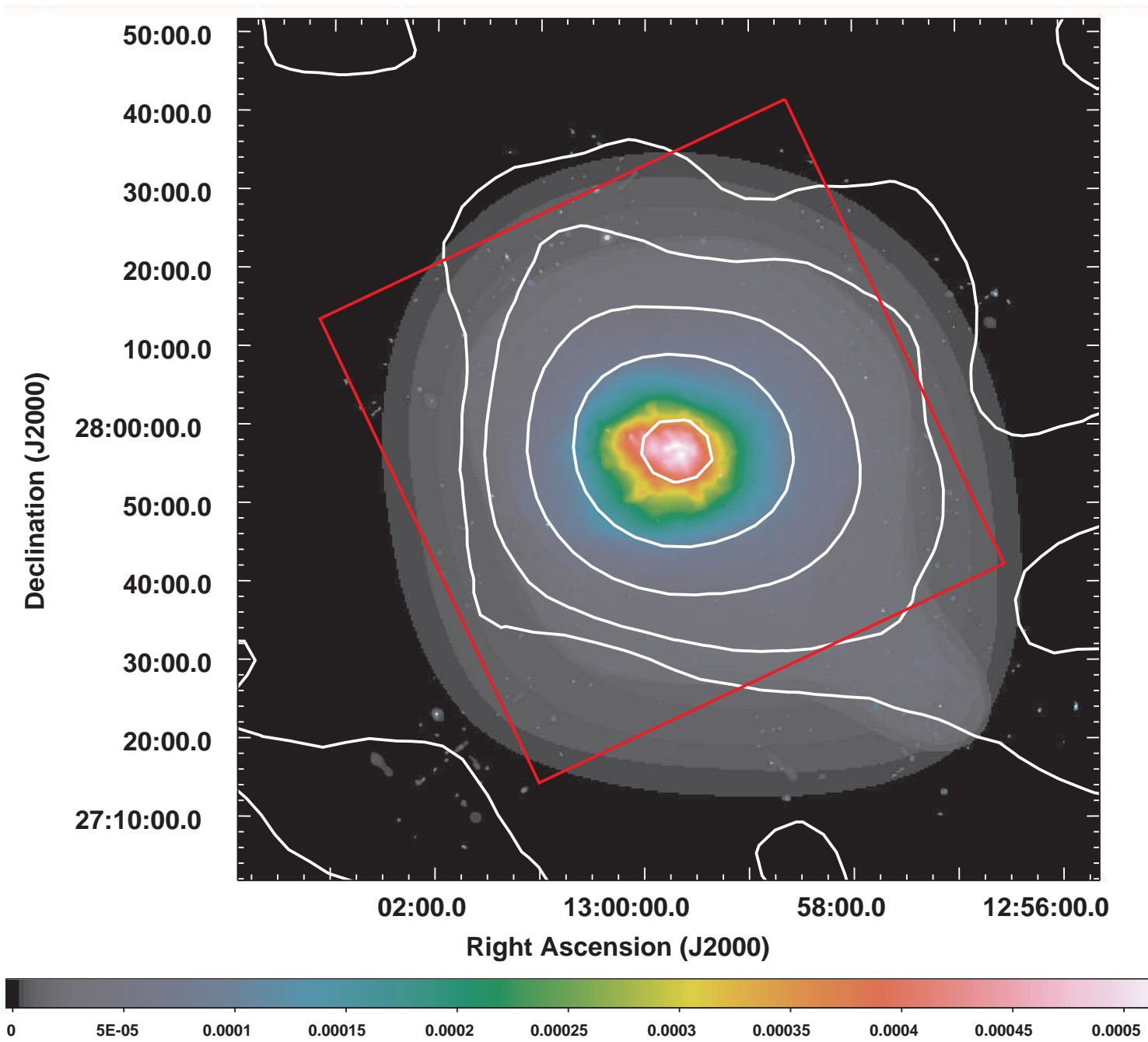

Fig. 4.1.- XMM-Newton EPIC-pn 2-7.5 keV wavelet-smoothed X-ray surface brightness image (Schuecker et al. 2004) with contours from the raw Swift BAT 14-20 keV survey image (square root spacing: $0.0,2.625 \times 10^{-6}, 1.05 \times 10^{-5}, 2.3625 \times 10^{-5}$, and $4.2 \times 10^{-5}$ counts $\left.\mathrm{s}^{-1} \mathrm{pix}^{-1}\right)$. Negative contours are not shown for clarity, and note that the FWHM of the BAT PSF is 19.5. The (red) box shows the $65.5 \times 65.5$ region from which the EPIC-pn spectrum is extracted for joint fits. The BAT emission is slightly more extended to the W-SW, as would be expected from the higher temperature gas in that direction. Note, however, that the outermost contour is consistent with noise and should be ignored. 
which is detailed in Section 4.3.1. Spectra extracted within a $16 \times 16$ contiguous grid of regions of size $4^{\prime} .3 \times 4^{\prime} .3$ are fit to single temperature APEC models over the energy range $0.5<E<14 \mathrm{keV}$.

While data from the Suzaku observation of Coma (OBSID 801097010) are not part of the current analysis, we do make use of the $2-7.5 \mathrm{keV}$ spectrum from the 0 chip of the X-ray Imaging Spectrometer (XIS0) for calibration purposes, as described in Section B.1. We use the same spectrum that served to cross-calibrate the data in the previous XMM-Newton/Suzaku analysis (region 10 from Wik et al. 2009).

\subsubsection{The Swift BAT 58-Month Survey}

The Swift mission is primarily to detect and localize gamma-ray bursts, which is accomplished with the very large FOV ( $\sim 1 / 8^{\text {th }}$ of the sky), coded mask aperture Burst Alert Telescope (BAT). As such, the nearly random pointing strategy culminates in an almost uniform coverage of the entire sky with an $\sim 8$ Ms of exposure time, made from many $\sim 5$ minute individual observations. Images of the sky are reconstructed by cross-correlating the shadow pattern of the randomly coded mask in front of the detectors with the detector pixels via a fast Fourier transform. The detectors are sensitive to hard X-ray/soft gamma-ray photons from 14-195 keV in 80 native energy channels. As part of the default survey processing, the channels are combined into 8 broader energy bands: 14-20 keV (E1), 20-24 keV (E2), 24-35 keV (E3), 35-50 keV (E4), 50-75 keV (E5), 75-100 keV (E6), 100-150 keV (E7), and 150-195 keV (E8). The final survey is built from the individual sky reconstructions, which are summed and resampled onto predetermined image planes of 6 facets, each in the Zenith Equal Area projection. The detailed processing methodology and survey properties for the 58-month BAT all sky survey are nearly identical to those described in Tueller et al. 
(2010) for the 22-month survey. The only major difference is that for the 58-month survey, the gain of each detector pixel was individually calibrated with an onboard radioactive source, which had not been done previously. This better accounts for the sensitivity of low gain pixels, effectively increasing the overall sensitivity. Also, the sky images are more finely resampled so that the pixels near the center of the image projection scale 2 .8 instead of $5^{\prime}$ as with previous versions of the survey. The main advantage of this change is to improve the centroiding of sources.

Because the systematic uncertainties in the survey-averaged spectrum of the Crab Nebula are smaller than the uncertainties in the BAT survey response matrix, BAT survey fluxes are tied to the Crab fluxes in each band (see Tueller et al. 2010, Sec. 4.5). One drawback to this approach is that a source flux is only guaranteed to be correctly determined if its spectrum within the energy band is identical the Crab's (a powerlaw with a photon index of $\Gamma \sim 2.1$ ), since the energy response within the band may not be uniform. Because we will fit the BAT spectrum jointly with the XMMNewton spectrum, it is important that the cross-calibration between the Swift BAT and XMM-Newton be accurate. Since the flux calibration of the BAT survey is based on the Crab spectrum, we have made sure that the Swift BAT and XMM-Newton agree on the flux and spectrum of the Crab. The cross-calibration between Swift BAT and XMM-Newton is discussed in detail in Appendix B.

\section{The Coma Cluster}

The 6 facets are oriented in Galactic coordinates with one facet centered on each of the Galactic poles and the other 4 centered uniformly around the Galactic plane. The Zenith Equal Area projection conserves surface brightness but not shapes, so objects far from the center of the projection can be somewhat distorted. However, 
the fortuitous location of the Coma cluster near the North Galactic pole, and thus the center of its facet, means that any such distortions are negligible. Nevertheless, for all parts of the analysis image pixels are referred to in terms of their Galactic coordinates so that any image projection effects are completely eliminated.

Hard X-ray emission is clearly detected in the first 4 energy bands, up to $50 \mathrm{keV}$. In Figure 4.1, we present the wavelet-smoothed 2-7 keV XMM-Newton EPIC-pn image of the Coma cluster mosaic overlaid with contours of the Crab-weighted BAT flux (see Tueller et al. 2010, for a description), which shows the hard X-ray emission to be elongated in the same East-West direction as the softer emission. As first noted by Ajello et al. (2009) in the 9-month BAT survey, the Coma cluster is partially resolved by the BAT, which is explicitly shown in Figure 4.2. This figure compares the radial surface brightness profile of Coma with that of a nearby point source with a comparable flux. Coma is clearly extended. The points plotted are individual pixels. The greater width of the distribution for Coma indicates that its surface brightness is not circularly symmetric. As shown in Figure 4.1, both the BAT and XMM-Newton X-ray emission is elongated in a ENE-WSW direction.

While coded masks instruments have some difficulty detecting extended emission, this is only true for emission extended on very large scales, when the shadow pattern of the mask on the detector plane becomes sufficiently blurred; for the BAT, simulations suggest that this scale, in principle, approaches the size of the FOV. The actual problem is that flux from each part of an extended source adds systematic noise to every other part of the source, eventually drowning the signal in a sea of noise. However, for small extensions this additional uncertainty is not overwhelming, especially if the rough flux distribution is already known. In Appendix C, we show that the Swift BAT provides accurate fluxes for extended sources on the scale of interest for 


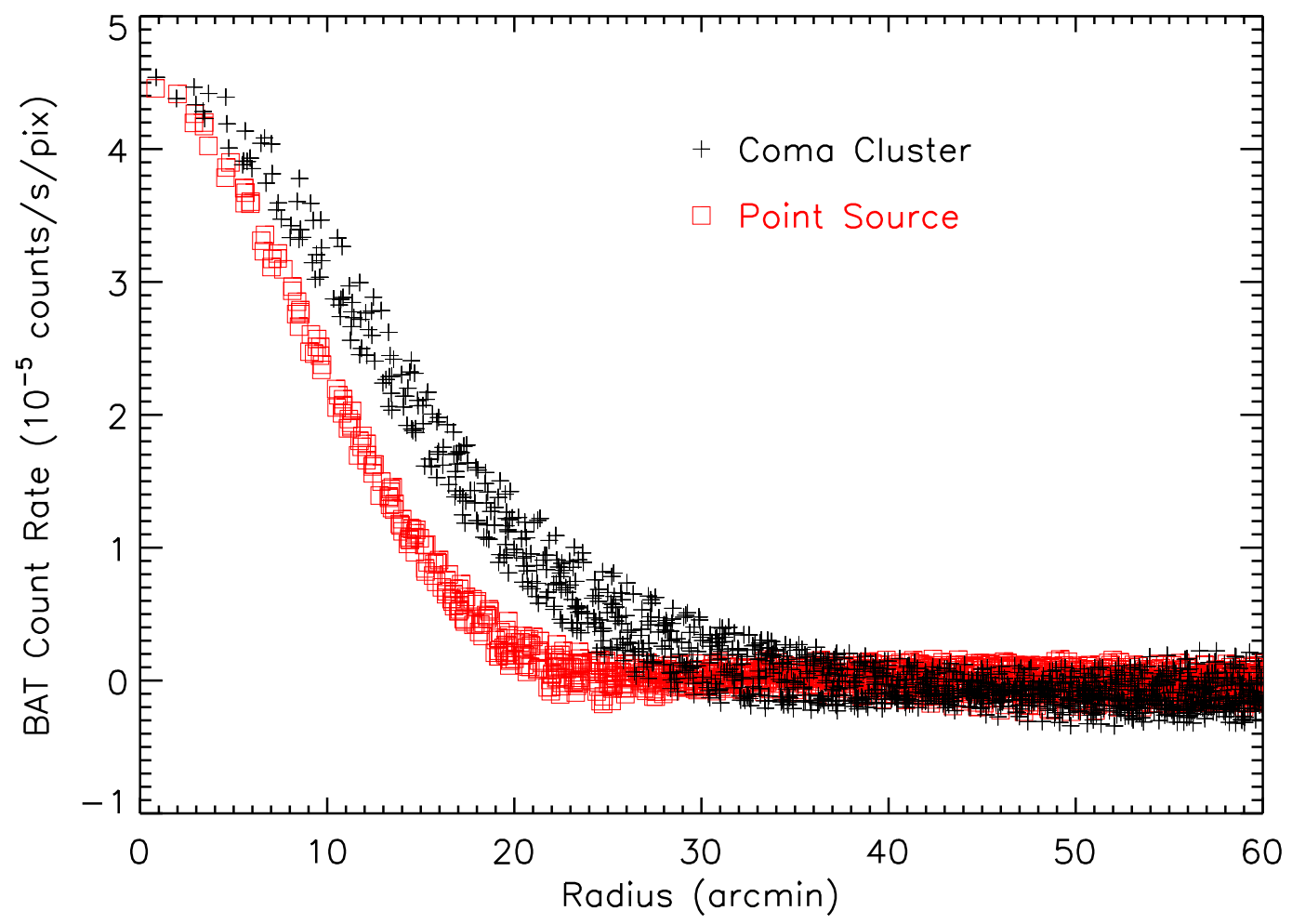

Fig. 4.2. - The radial profile of the Coma cluster (crosses, black) compared to a point source of comparable brightness (squares, red; scaled slightly to match Coma's central flux). Each point represents an individual pixel. The BAT emission from Coma is clearly extended and not axially symmetric, as shown by the the larger spread in pixel count rates in its profile compared to the point source. 
the Coma cluster.

Because nearby pixels in the BAT survey images are correlated, it is more difficult to determine the flux uncertainties for extended sources in the BAT than is usually

the case for X-ray images. For point sources, the flux uncertainties can be determined from the fluctuations in the local background in the BAT survey images. For Coma, we calculate the RMS fluctuations in the background $\left(\sigma_{\mathrm{bgd}}\right)$ around Coma in an annulus of radius $15<r<100$ pixels $\left(42^{\prime}<r<4.67\right.$ ), as is typically done for sources in the BAT survey. These values, and the flux uncertainties for extended sources like Coma, are derived in Appendix D.

\subsection{Characterization of Extended Emission in BAT Images}

To extract fluxes for extended sources, we choose to test a priori model distributions, as opposed to using a method like the "CLEAN" algorithm (Högbom 1974), which reconstructs fluxes from an unknown underlining distribution assuming the PSF shape only. (See Appendix C.2 for details about this choice.) We represent a diffuse source as a collection of point sources, each of which is convolved by the PSF (Eqn. C.1) and summed together. Throughout this work, image data are fit to these spatial models using the MPFIT algorithm (Markwardt 2009), which performs a LevenbergMarquardt least-squares minimization to converge on best-fit parameter values.

\subsubsection{Model Spatial Distributions of Hard X-ray Emission}

Our goal is to detect IC emission from the same electrons producing the radio halo; however, the electron spatial distribution need not follow the radio halo if $B$ varies 
spatially within the ICM. Indeed, there is evidence that the magnetic field in Coma declines with radius (Bonafede et al. 2010), which would allow for a more extended relativistic electron population visible through IC interactions with CMB photons. To accurately search for this potential signal, we need to both choose model distributions for this emission and to fully model the thermal emission also present in the BAT energy bands.

\section{Thermal Models}

Following the success of the $X M M$-Newton-derived temperature map for explaining the thermal origin of the Suzaku HXD-PIN spectrum (Wik et al. 2009), we use the same map to predict the spatial distribution of thermal emission at hard energies. For each region of the map, the flux of the best-fit APEC model is calculated in each BAT energy band and treated as a point source at that location. Note that because the APEC and MeKaL models are not defined above $50 \mathrm{keV}$ in XSpec, we use MeKa to derive the temperature map fluxes in the 4 highest energy bands. Then, for each band the 232 temperature map region "points" are taken together to serve as the diffuse model. To compare this or any of our diffuse models to the BAT image data, each point is assigned the PSF shape with its peak value equal to the point flux, and the overlapping PSFs are summed together and sampled at the location of the image pixels.

Thermal emission is detected in the first 4 BAT energy bands E1-E4. Since this emission is an extension of the X-ray emission which dominates the XMM-Newton image and its distribution is known, we fix the location of the thermal model to the best-fit position of the model for the E1 band data, where the signal-to-noise ratio is the highest. 
Note that for the thermal model "fits" to the Swift BAT spatial distribution in various bands, only the normalization of the model in each band is allowed to vary. The spatial distribution within each of the bands is completely determined from the $X M M-N e w t o n$ data.

The XMM-Newton data will also contain any nonthermal emission within the $X M M-N e w t o n$ band. Is it reasonable to use the $X M M$-Newton temperature map and image to determine the spatial distribution of the hard X-ray thermal emission? The spectra in the temperature map were fit over the energy range $0.5 \mathrm{keV}<E<14 \mathrm{keV}$. While the upper limit of this band is fairly hard, the low energy limit guarantees that the spectra are dominated by softer photons. For any sensible nonthermal spectrum, the $X M M$-Newton spectra are dominated by thermal emission. In fact, if there is cool, dense gas along a given line of sight, the XMM-Newton-based model may actually underestimate the thermal hard X-ray emission. In any case, if there is strong nonthermal emission in the XMM-Newton spectrum, it will dominate the harder BAT energy bands, and will be uncovered in the joint spectral fits to the XMM-Newton and BAT spectra (Section 4.4 below).

\section{Nonthermal Spatial Models}

The Suzaku HXD-PIN upper limit, which is 2.5 times below the RXTE and Beppo$S A X$ detections, only excludes those measurements if the IC emission originates from a relatively compact region $\left(R \lesssim 20^{\prime}\right)$. More extended emission of roughly uniform surface brightness, however, would be consistent with both the detections and upper

limit. In most physical models for the IC, it is likely that he surface brightness would decline with radius; one exception is the KW model discussed below. However, there is no single well-established model for this decline. Since our object is to test 
the possibility that the difference between our Suzaku HXD-PIN upper limit and the $R X T E$ and Beppo-SAX detections is due to the extent of the IC emission, we consider the extreme case of a uniform surface brightness disk. Thus, we assume any nonthermal emission to take the form of a uniform brightness, circular disk with a radius $R=25^{\prime}$ (R25), 30' (R30), 35' (R35), 40' (R40), 45' (R45), or 60' (R60).

Recently, Kushnir \& Waxman (2010) proposed another model for the IC emission of the Coma cluster which is consistent with both the Suzaku HXD-PIN upper limit and the $R X T E$ and Beppo-SAX detections. In this model, the IC hard X-ray emission comes from a separate population of electrons from those in the radio halo. This new population of electrons are accelerated at the virial accretion shock of the cluster at a very large radius. These virial shock accelerated electrons lose energy quickly, and form a shell of hard X-ray emission, which projects on the sky as a ring with nearly uniform surface brightness emission in its interior. We will refer to this as the KW model. While most of the flux resides in a ring at the cluster virial radius, the amount of flux detected by an instrument pointed at the cluster center will depend sensitively on its FOV. We take all the model parameter values for Coma directly from Kushnir \& Waxman (2010) when comparing their expected flux to the constraints imposed by the BAT data, though the only relevant parameter for the spatial distribution is the accretion shock radius $\theta_{200}=82^{\prime} \cdot 1$. The radial distribution of flux is simply geometrical in form, assuming an infinitely thin shell at this radius; the expression is given in Kushnir \& Waxman (2010, Eqn. 9).

In reality, it is unlikely the spatial distribution of emission would be as regular and axisymmetric as portrayed by these models. However, for the spatial extent we consider relative to the resolution of the BAT, deviations from the idealized models will not particularly impact our results. 
We do not assume that the center of the nonthermal emission distribution from

Coma is the same as the center of the thermal emission. Instead, for each of the nonthermal models, the model center is placed at 81 different positions on a $9 \times 9$ grid with 2'.5 spacings around the centroid of the large-scale thermal emission. The center of the nonthermal emission is taken as one of the parameters to be varied in the fits of the spatial and spectral distributions below.

\subsubsection{Spatial Fits to the BAT data}

The spatial models for the thermal emission alone, or for the thermal emission plus nonthermal emission, were fit to the pixel values in the BAT images in each of the 8 BAT bands. As noted above (Section 4.3.1), the center of the thermal model was determined by fitting the center in the E1 band. For the thermal model, only the overall normalization was allowed to vary. For the nonthermal models, the center was varied for a grid of positions (but fixed in each individual fit, see Section 4.3.1). The model normalization (i.e., flux) in each of the 8 BAT bands was fit independently for the thermal and nonthermal models. That is, the spatial fits made no assumptions about the spectrum of either type of emission.

In Figure 4.3, we present the Swift BAT images of Coma in all 8 energy bands (first and third columns) along with the thermal model-subtracted residuals for bands E1-E4 (center column). The spatial distribution of the thermal models is represented with the contours in the first column. Note that only positively-valued pixels appear in the grayscale, which has a square-root scaling, and that for each band pure black corresponds to a slightly different value. Each panel covers an identical $2.7 \times 1.5$ region of the sky. Residuals from the fits are consistent with background fluctuations, as can also be seen in the radial profiles shown in Figure 4.4. 


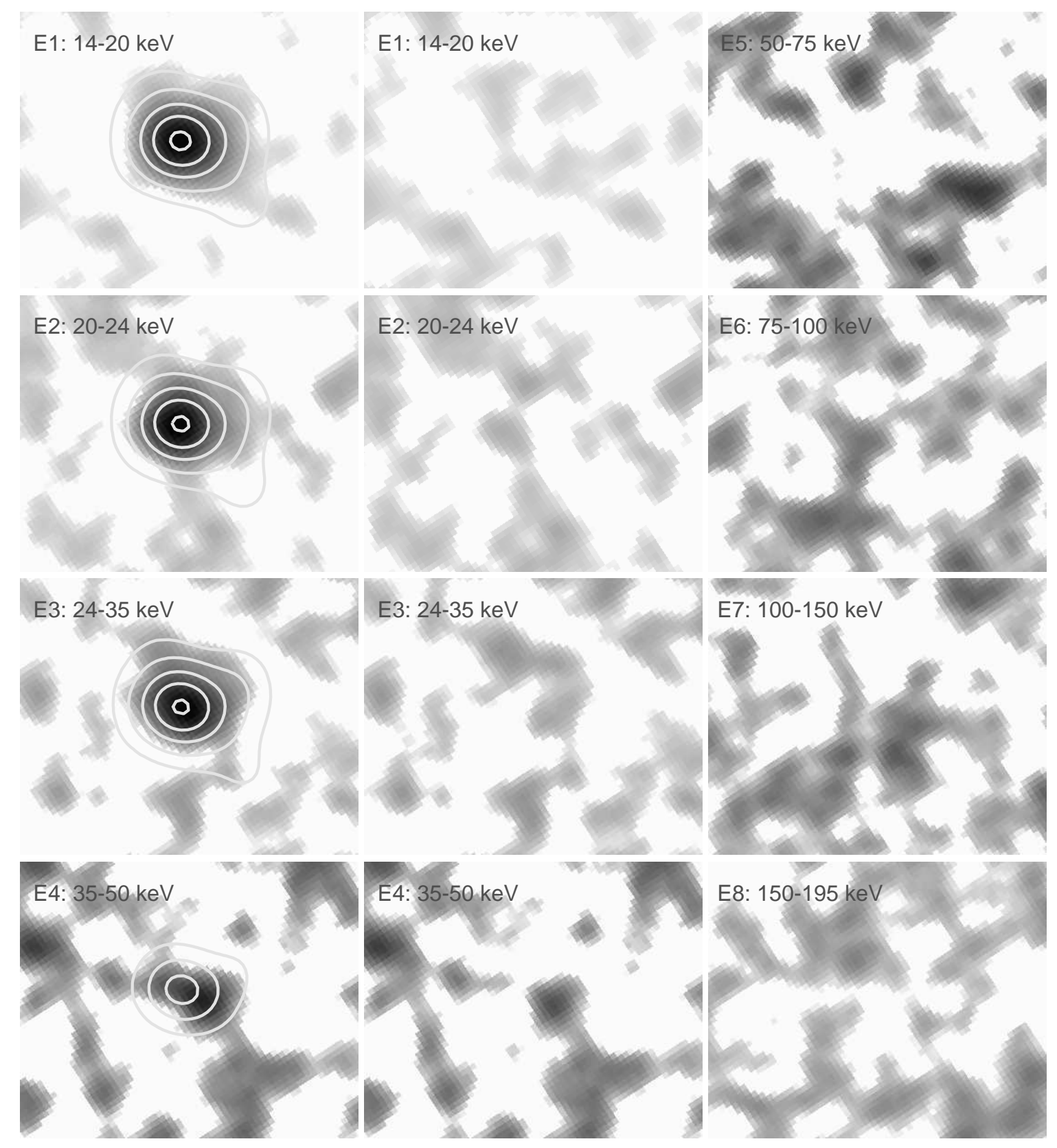

Fig. 4.3.- Images from the 8 energy bands of the Swift BAT survey (first and third columns). The greyscale follows a square root scaling from 0 counts/s (white) to $\left[>4.2 \times 10^{-5}(\mathrm{E} 1),>1.7 \times 10^{-5}(\mathrm{E} 2-\mathrm{E} 3),>5 \times 10^{-6}(\mathrm{E} 4-\mathrm{E} 8)\right] \operatorname{counts~s}^{-1} \mathrm{pix}^{-1}$ (black). The contours in the images in the first column show the best-fit thermal model for each band and follow a square root spacing from 0 to the maximum of the greyscale for that band, with 5 contours. For E4, only three contours are shown as the model is fainter than the brightest region of the data to the west of the cluster center. For E1, the contours occur at the same levels as shown in Fig. 4.1). The middle column shows the thermal model-subtracted residual images for E1-E4, with the same for greyscale as the data on the left. The residuals show that the thermal spatial models are generally well-mapped to the actual data. Note that the background is also fit for and subtracted from the data in the residual images, so the outer fluctuations are not identical to those in the left column. 

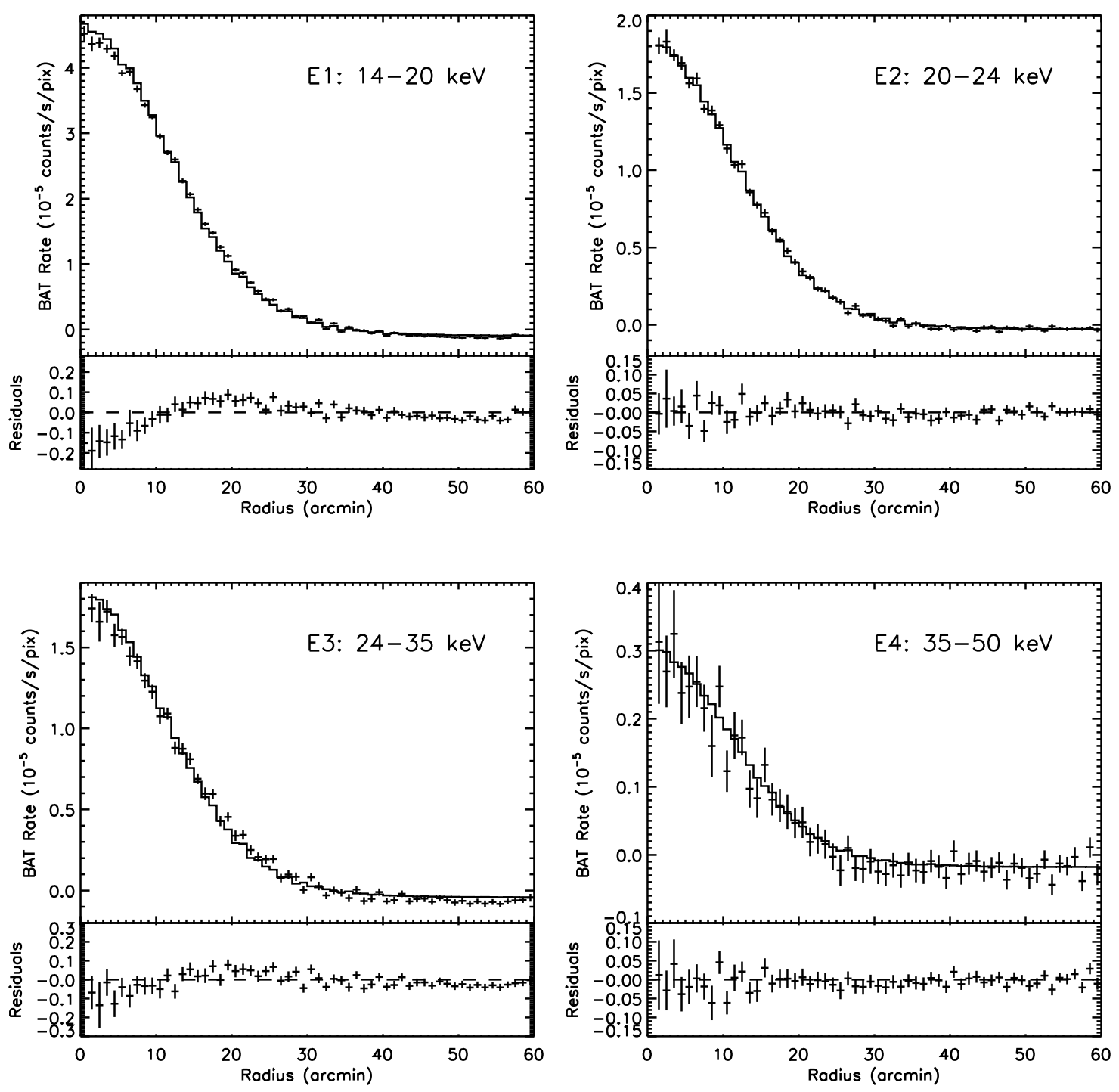

Fig. 4.4. - Radial profiles of the data and thermal model fits shown in the first column images of Fig. 4.3. BAT pixels are averaged in annuli of $1^{\prime}$ width (crosses), as are the model values for each pixel position (histogram); the residuals are plotted below each fit, on the same scale as the fit. The structure in the E1 and E3 residuals could be due to a slightly larger PSF FWHM and/or a true spatial distribution of emission that differs slightly from our models; in either case, the effect on the extracted flux would be less than its $1 \sigma$ error. 
As noted above (Section 4.3.1), these thermal models are solely based on the $X M M-N e w t o n$ temperature map. That is, the spatial distribution is completely determined from the $X M M-N e w t o n$ data, and only the normalization is allowed to vary. Still, this thermal model provides an adequate description of both the spatial and spectral (discussed in Section 4.4 below) properties of the detected emission in the BAT data. This success justifies our approach and confirms that extended emission is detected with the same efficiency as that from the cluster center.

The good fit of the XMM-Newton-based thermal model for the emission in BAT bands E1-E4 and the lack of obvious excess emission in the harder E5-E8 bands suggests that nonthermal emission is not very strong or extended. The nonthermal model with the most extended emission is the KW model (Kushnir \& Waxman 2010), in which the IC hard X-ray emission comes from a thin shell at a very large radius. Following the methodology in Section C.2, we simulate the expected combined thermal and nonthermal flux distribution for this model, and compare to the actual data. The results for the E1 BAT band are shown in the upper panel of Figure 4.5. Clearly, we do not detect the nonthermal emission expected by the KW model. The lower panel shows the thermal plus KW model compared to a simulation of the BAT data assuming the distribution actually followed this model. It is clear that the statistics in the BAT data would allow us to detect the nonthermal emission from the KW model, were it present. A more quantitative limit is derived in Section 4.5.

\subsection{Spectral Fits}

To search for a nonthermal component in the X-ray spectrum of the Coma cluster, spectral models are fit to the data. The fits were done for the Swift BAT spectrum alone, or simultaneously with the XMM-Newton spectrum of the cluster. The BAT 


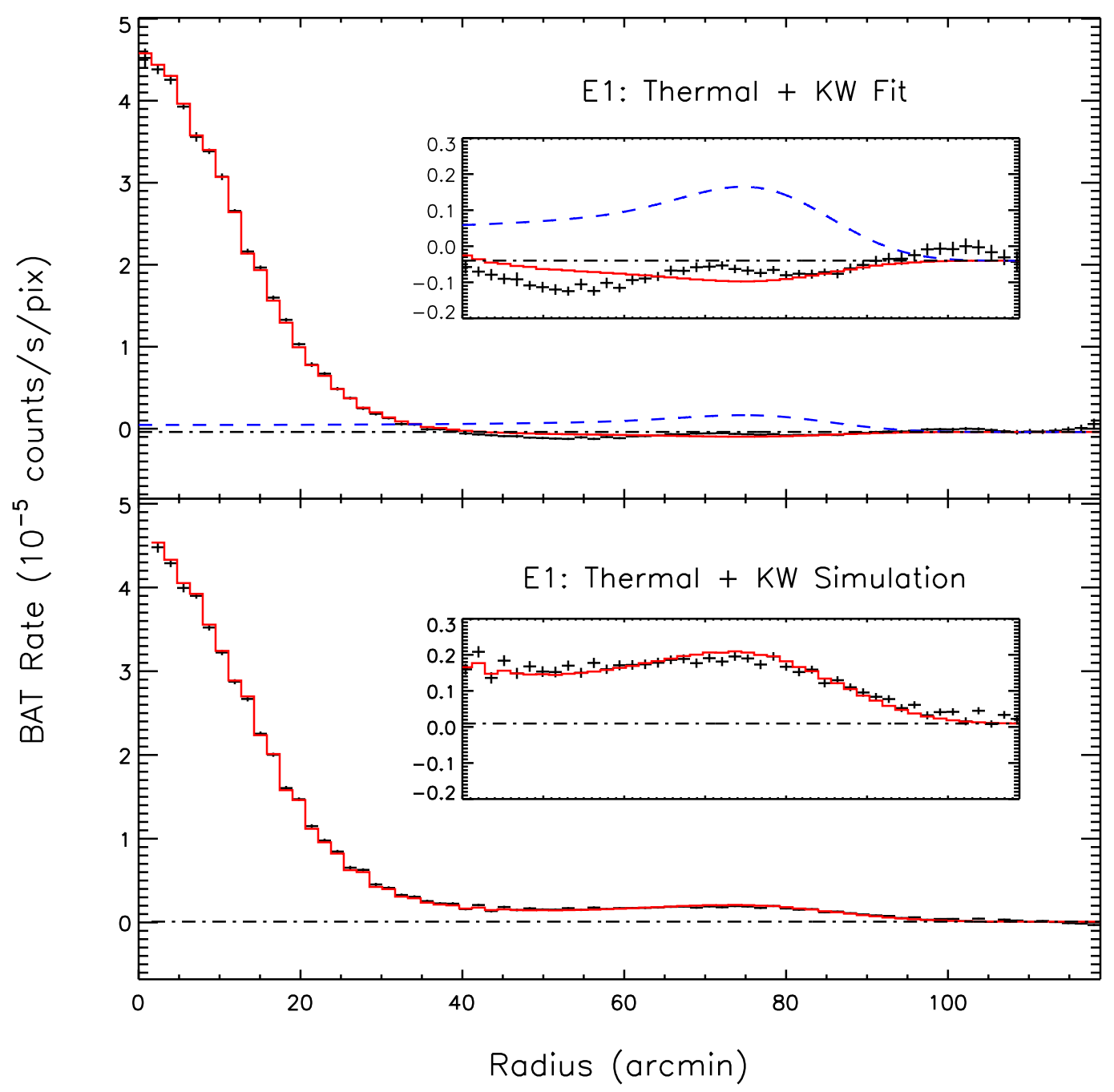

Fig. 4.5. - The top panel shows the BAT E1 profile and spatial fit for a model with both thermal emission and nonthermal emission following the KW model (histogram, red). The best-fitted value of the normalization of the KW component is actually negative. The dash-dot line represents the background level and the dashed line (blue) shows the predicted spatial distribution of flux for the KW model (Kushnir \& Waxman 2010) from 14-20 keV. The inset expands the scale of the y-axis above it to highlight the difference between the data and the expected flux. In the bottom panel, we perform the same fit to simulated BAT data based on the thermal plus KW model, including shot and systematic noise comparable to that present in the actual data. This shows that the BAT would have easily detected a nonthermal component with the spatial distribution given by the KW model and the predicted flux. 
spectra were binned into 8 spectral channels, given by the 8 standard BAT bands E1-E8. To determine the spectrum in each channel, the total measured raw photon fluxes in each band were converted into "true" photon fluxes using the calibration determined from the BAT Crab spectrum; we also create a generic redistribution matrix to better represent models with spectral shapes that differ from the Crab (see Section B.1). The uncertainties in each channel were determined from the flux uncertainty for a point source $\sigma_{\text {bgd }}$ (see Section D.1), and then corrected for the effects of source extent as described in Section D.2, Equation (D.3), and Table B.1. The final uncertainty is given by $\sigma_{\text {diffuse }}$ in Equation (D.3). Additionally, a problem with the implementation of the APEC, which we use as our thermal description for spectral fitting, and MeKaL models in XSpec is that they are undefined above $50 \mathrm{keV}$. Therefore, for the 4 energy channels above $50 \mathrm{keV}$, we substitute MeKa for APEC. This should have no significant effect given the small thermal flux at these energies relative to the errors.

For the BAT-only spectra, in which only the nonthermal component of the spatial fit is used to build the spectra, a single power law model is sufficient to measure the nonthermal flux. We also do joint fits of the Swift BAT (containing both the thermal and nonthermal spatial components) and XMM-Newton spectra. In these fits, the excellent statistics at low energies in the XMM-Newton spectra very strongly constrains the thermal emission. However, the $X M M-N e w t o n$ mosaic covers a smaller area compared to either the Swift BAT or most of the nonthermal spatial models. (The XMM-Newton extraction region used for this spectral analysis is indicated by the square in Figure 4.1.) Thus, in these fits the models applied to the XMM-Newton spectra are reduced by the fraction of the emission in our XMM-Newton spectral extraction region. 


\subsubsection{Joint $X M M-N e w t o n$ EPIC-pn - Swift BAT Thermal Emis- sion Fit}

We first consider a purely thermal model for the X-ray emission in Coma, and fit the Swift BAT and XMM-Newton spectra simultaneously. The spatial distribution of the emission was assumed to follow the thermal model (Section 4.3.1) as determined from the $X M M-N e w t o n$ data. The resulting single temperature fit is presented in Figure 4.6, and the parameters are given in the first row of Table 4.1. The quality of the fit is quite good, indicating that a single component description for the temperature structure is sufficient and that the spectra have been reasonably well cross-calibrated. A slight ascending trend in the E1-E3 residuals exists, however, which is primarily due to a slightly lower than expected E1 flux. While not particularly significant, it is worth mentioning several potential causes for the trend. The most straightforward explanation is that the calibration is slightly wrong. We presume the true Crab spectrum to be a simple power law across the entire 2-200 keV interval, but if the spectrum actually steepens around $E \sim 10 \mathrm{keV}$ as is likely the case (Kirsch et al. 2005), the higher energy bands will be assigned progressively higher flux conversion factors (column 3 in Table B.1); basically, the flux in an energy band will be more and more overestimated for bands at higher and higher energies. Also, because emission is more significantly detected in the lower energy bands, a small change in the overall value of the XMM-Newton and Swift cross-normalization factor - such that the Swift flux would be raised - could reduce the spread in residuals. From a more physical perspective, a single temperature model is not entirely appropriate; in multi-temperature model fits, the trend in residuals is not as strong. In any case, an adjustment to the cross-calibration of $3-5 \%$ is sufficient to account for the trend, which is well within our assumed $90 \%$ confidence interval uncertainty of $10 \%$. 


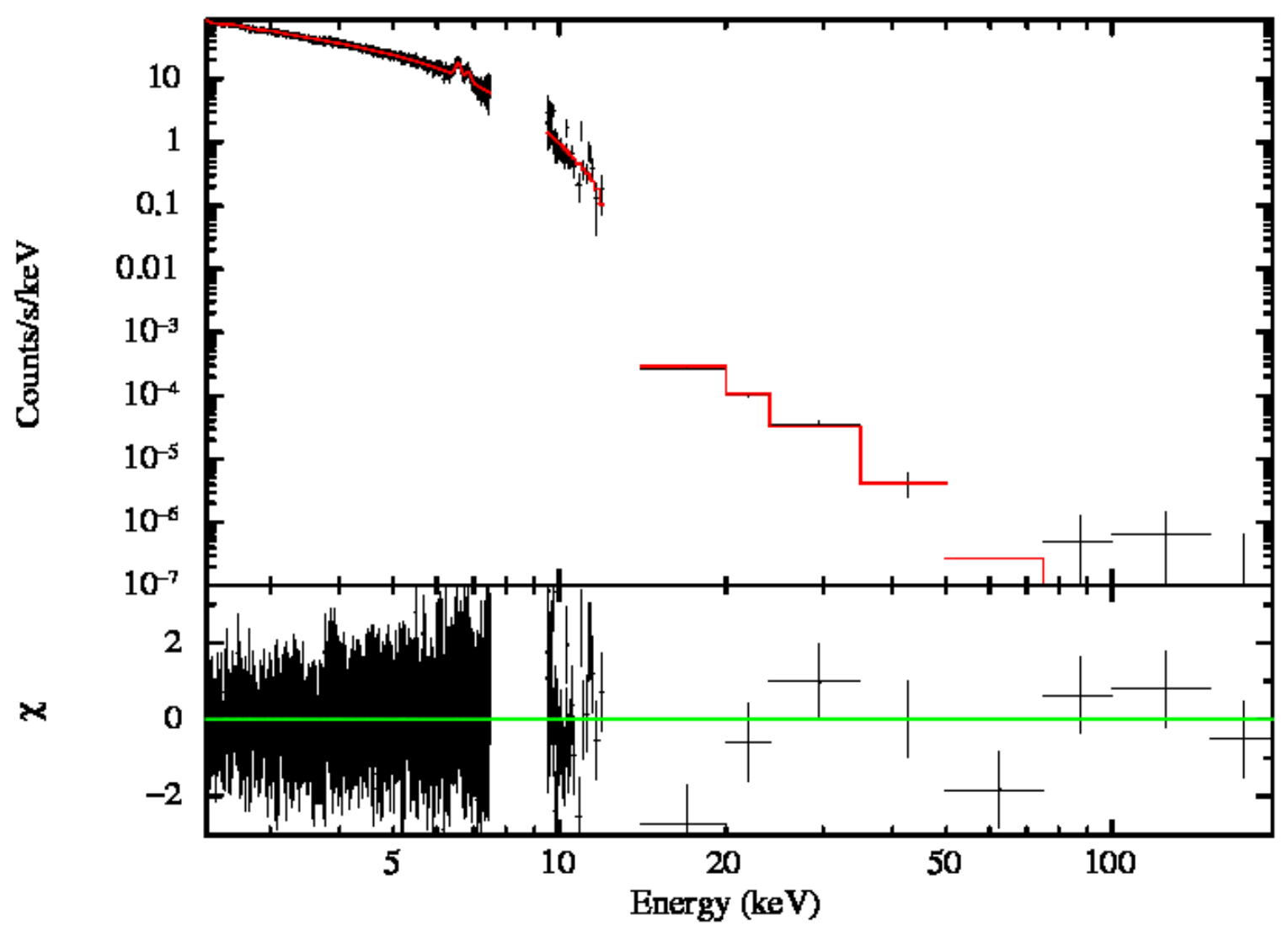

Fig. 4.6. - Single temperature (APEC) fit to the XMM-Newton EPIC-pn and Swift BAT spectra. The BAT spectra shown are reduced to the fraction which occurs in the $X M M-N e w t o n$ spectral extraction region. The BAT spectra were constructed assuming the spatial distribution predicted by the XMM-Newton temperature map. The origin of the low E1 (14-20 keV) flux is discussed in the text. A single temperature model $(k T=8.24 \mathrm{keV})$ is sufficient to describe the $2-200 \mathrm{keV}$ emission from the central square degree region of the Coma cluster. 
Table 4.1. Joint Fits to XMM-Newton and Swift Spectra

\begin{tabular}{llccccc}
\hline \hline $\begin{array}{l}\text { Spatial } \\
\text { Model }\end{array}$ & $\begin{array}{l}\text { Spectral } \\
\text { Model }\end{array}$ & $\begin{array}{c}k T \\
(\mathrm{keV})\end{array}$ & $\begin{array}{c}\text { Norm. } \\
\left(\mathrm{cm}^{-5}\right)\end{array}$ & $\begin{array}{c}\Gamma \text { or } k T \\
\mathrm{~b}\end{array}$ & Norm. & $\chi^{2} /$ dof \\
\hline Thermal Region & Single $\mathrm{T}$ & $8.28 \pm 0.13$ & $0.373 \pm 0.002$ & - & - & $1576.79 / 1544$ \\
Thermal Region & $2 \mathrm{~T}^{\mathrm{d}}$ & 7.8 & 0.25 & 9.4 & 0.12 & $1575.77 / 1542$ \\
Thermal Region & $\mathrm{T}_{\text {map }}$ & - & - & - & - & $1590.01 / 1545$ \\
Thermal Region & $\mathrm{T}+\mathrm{IC}$ & $8.27 \pm 0.13$ & $0.373 \pm 0.003$ & 7.2 & $<0.51$ & $1576.79 / 1543$ \\
Thermal Region & $\mathrm{T}+\mathrm{IC}^{\mathrm{f}}$ & $8.27 \pm 0.13$ & $0.373 \pm 0.003$ & 2.0 & $<0.00148$ & $1577.21 / 1543$ \\
Thermal \& KW & Single T & $8.30 \pm 0.13$ & $0.373 \pm 0.002$ & - & - & $1570.32 / 1544$ \\
Thermal \& KW & $2 \mathrm{~T}^{\mathrm{e}}$ & 7.8 & 0.25 & 9.7 & 0.12 & $1568.63 / 1542$ \\
Thermal \& KW & $\mathrm{T}_{\text {map }}$ & - & - & - & - & $1584.59 / 1545$ \\
Thermal \& KW & $\mathrm{T}+\mathrm{IC}$ & $8.30 \pm 0.14$ & $0.372 \pm 0.002$ & -1.5 & $<1.9 \times 10^{-9}$ & $1570.23 / 1543$ \\
Thermal \& KW & $\mathrm{T}+\mathrm{IC}$ & $8.30 \pm 0.14$ & $0.373 \pm 0.003$ & 2.0 & $<0.00082$ & $1570.62 / 1543$ \\
\hline
\end{tabular}

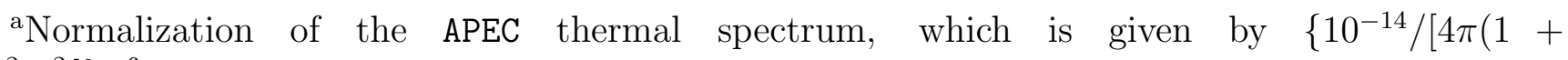
$\left.\left.z)^{2} D_{A}^{2}\right]\right\} \int n_{e} n_{H} d V$, where $z$ is the redshift, $D_{A}$ is the angular diameter distance, $n_{e}$ is the electron density, $n_{H}$ is the ionized hydrogen density, and $V$ is the volume of the cluster.

${ }^{\mathrm{b}}$ Value is $\Gamma$ for the $\mathrm{T}+\mathrm{IC}$ model and $k T$ (in $\mathrm{keV}$ ) for the $2 \mathrm{~T}$ model.

${ }^{c}$ Value is the normalization of the power-law component for the $\mathrm{T}+\mathrm{IC}$ model, which is the photon flux at a photon energy of $1 \mathrm{keV}$ in units of photons $\mathrm{cm}^{-2} \mathrm{~s}^{-1} \mathrm{keV}^{-1}$. For the $2 \mathrm{~T}$ model, the value is the normalization of the second APEC thermal model in units of $\mathrm{cm}^{-5}$.

dParameters unconstrained.

${ }^{e}$ Value of $\Gamma$ is fixed when deriving errors.

${ }^{\mathrm{f}}$ Value of $\Gamma$ fixed based on radio spectrum (Giovannini et al. 1993). 
The best-fit temperature is $k T=8.24 \pm 0.12$ (stat) \pm 0.15 (sys) keV. The systematic term in the error is based on varying the cross-normalization factor by $10 \%$; the origin of this percentage is discussed in Section 4.5 below. This global temperature matches extremely well with previous measurements. For example, Hughes et al. (1993) found $k T=8.21 \mathrm{keV}$ with a spectrum spanning $\sim 2<E<11 \mathrm{keV}$ from the Ginga satellite, which had a similar FOV (collimator with 1-2 ${ }^{\circ}$ FWHM) to our aperture, and Arnaud et al. (2001) found $k T=8.25 \mathrm{keV}$ over a smaller FOV and lower energy range $(0.3<E<10 \mathrm{keV})$ with the XMM-Newton EPIC-MOS instruments. Including lower energy photons tends to lower single-temperature fits to multi-component spectra (Cavagnolo et al. 2008b), and having a smaller FOV tends to emphasize the hotter central temperature of $k T \sim 9 \mathrm{keV}$ in Coma, which both explains why the Ginga and $X M M$-Newton temperatures agree and why the XMM-Newton-Suzaku analysis of Wik et al. (2009) found a slightly higher $k T=8.45 \mathrm{keV}$. While we find good agreement with other measurements, note that our temperature, along with other temperatures derived with XMM-Newton, could be systematically cooler by a few tenths of a keV than temperatures obtained with other observatories, given the steeper XMM-Newton Crab spectrum and the implications for its instrumental response (see Section B.1).

We also tried a two-temperature thermal model for the XMM-Newton and BAT data. However, the two temperatures and normalizations could not be individually constrained by the data (Table 4.1); the temperatures/normalizations listed in the table result when the two-temperature model is fit for with initial temperatures of $k T_{1}=6 \mathrm{keV}$ and $k T_{2}=10 \mathrm{keV}$. The two-temperature fit is not a significant improvement on a single temperature fit.

While the average spectrum in the square degree region around Coma is adequately described with one or two temperatures for the gas, in fact the temperature 
distribution is quite non-isothermal. We account for temperature variations in the spatial models used to extract fluxes from the BAT images by extrapolating the $X M M$ Newton temperature map from Wik et al. (2009) to higher energies. This map can also be converted into a spectral model (labeled " $\mathrm{T}_{\mathrm{map}}$ " in Table 4.1) and compared to the joint spectrum. By including the spatial information of the XMM-Newton data in the spectral model, we can better account for the thermal contribution in the BAT energy bands. The quality of this fit (allowing the normalization, but not the shape, of the model to vary) is reported in Table 4.1. In principle, the " $\mathrm{T}_{\text {map }}$ " spectral model should perfectly represent the total $X M M$-Newton spectrum, but due to incomplete coverage of the temperature map with the total $X M M$-Newton region and the imperfect determination of the individual temperatures, the $\chi^{2}$ value - which is primarily driven by the higher quality XMM-Newton data - is larger than for the other fits, in which the model shape is free to vary and can account for these minor differences. Even so, the BAT data are slightly better described by this model than by any of the other spectral models presented. Although this result is perhaps expected, given that the "T $\mathrm{T}_{\text {map }}$ " spatial distribution is used to measure the BAT fluxes, it does indicate that the method is self-consistent.

In all of the thermal models investigated, no evidence for a strong high-temperature component is hinted at by the BAT data.

\subsubsection{Nonthermal Spectra}

\section{Nonthermal Emission from the Cluster Center}

To search for evidence of more centrally located nonthermal emission, an IC component was first fit to purely thermally-derived spectra - i.e., spectra created from fits to the BAT data using only the thermal spatial model - which are reported in rows 
4 and 5 of Table 4.1. As in Section 4.4.1, all fits are to the joint XMM-Newton-Swift spectrum extracted from the square region in Figure 4.1. Not surprisingly, the thermal model parameters are almost identical to the fits without the IC component, and no significant IC emission is present. The good single temperature fit to the Swift BAT and XMM-Newton data already suggests that the nonthermal contribution is not very significant. If the photon spectral index $\Gamma$ of the nonthermal component is allowed to vary, it is unconstrained and the best-fitted value is unphysically steep, and in any case, only an upper limit can be placed on the nonthermal flux (Table 4.1). If we assume a photon index of 2.0 for the nonthermal component based on the radio data (Section 4.5 below), the $90 \%$ upper limit on the $20-80 \mathrm{keV}$ flux is $1.24 \times 10^{-12}$ $\operatorname{ergs} / \mathrm{s} / \mathrm{cm}^{2}$, which corresponds to $<0.8 \%$ of the total flux in the range $2<E<200$ $\mathrm{keV}$.

\section{Extended Nonthermal Emission}

Our goal is to search for extended nonthermal emission, which is measured with spatial model fits to the unlimited FOV BAT survey images (Section 4.3.2). Because the thermal and nonthermal spatial model normalizations are individually and simultaneously allowed to vary to best match the total flux in the BAT images, spectra can be created from the sum of both components or separately, and also within any aperture. For each of the 81 grid positions relative to the center of the cluster at which each nonthermal model was fitted for, two spectra are produced. One consists of the total, unvignetted flux of the nonthermal component only, and the other includes the sum of both the thermal and nonthermal emission inside the $X M M$-Newton extraction region. The second type of spectrum has the advantage that it can be jointly fit with the EPIC-pn spectrum, which in the case of a non-detection provides a tighter constraint 
on the flux of nonthermal emission than the first type, since the nonthermal component must be consistent with the higher quality, lower energy XMM-Newton data as well. In none of these cases, for either type of spectrum, is a nonthermal component detected with $\gtrsim 2 \sigma$ confidence. We therefore conclude that, while the Swift BAT instrument is certainly sensitive to extended emission, none of a nonthermal origin is observed in the current version of the survey. As an example, the fit parameters for various spectral models are shown in Table 4.1 for the KW spatial model nominally positioned (i.e. centered on the large-scale XMM-Newton emission).

In Figure 4.7, the nonthermal model with the most significant IC component is shown, assuming a fixed photon index $\Gamma=2$ for the spectral fits of each nonthermal spatial distribution tried. Note that the model in this figure represents the upper limit for a nonthermal component, not its best-fit value, and that the cross-normalization has been adjusted by $10 \%$ in the direction that favors a nonthermal signal. The BAT spectra in Figures 4.6 and 4.7 are quite similar, indicating that even in the most favorable case the data reject a significant IC contribution to the spectrum of the Coma cluster, extended or otherwise.

\subsection{Upper Limits to Diffuse, Nonthermal Emission}

To ensure appropriate upper limits are derived, we must determine and include any important systematic uncertainties in our results. Typically, instrumental and/or cosmic backgrounds can be a serious concern and must be carefully treated. At lower energies where the thermal emission is bright, the background is not comparable to cluster emission until an energy of $\sim 7 \mathrm{keV}$, so even a background uncertainty of a few percent does not significantly impact the XMM-Newton EPIC-pn spectrum. This background includes both the non-X-ray and cosmic backgrounds. Given the 


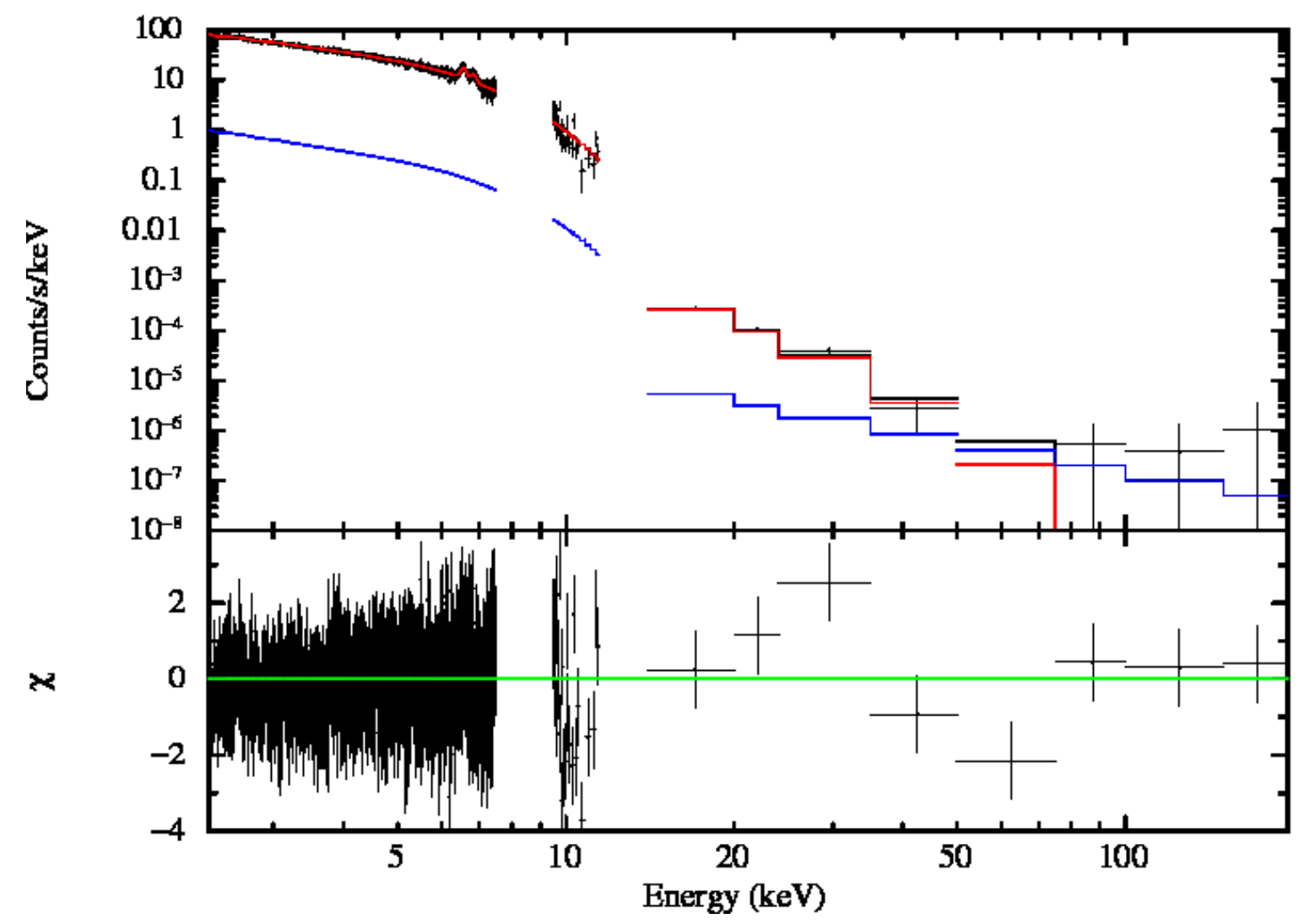

Fig. 4.7. - Thermal plus nonthermal model fit to the Swift and XMM-Newton data for the $\Gamma=2$ power law nonthermal model corresponding to the $90 \%$ upper limit, including the systematic uncertainties as described in the text. This example, which is the model with most significant nonthermal flux, is for a $25^{\prime}$ radius, uniform surface brightness disk of nonthermal emission with a position offset from the center of the large-scale thermal emission by -2.5 and $10^{\prime}$ in $l$ and $b$, respectively. As is true of all the upper limits from the joint spectra, the nonthermal component does not exceed $\sim 1 \%$ of the low energy part of the XMM-Newton spectrum, nor does it compete with the thermal emission until energies $E \gtrsim 50 \mathrm{keV}$. 
size of the mosaic, the uncertainty in the overall EPIC-pn background used here $(\sim 2$ $12 \mathrm{keV}$ ) is $2.4 \%$ (Read \& Ponman 2003). Increasing the XMM-Newton background favors larger nonthermal fluxes since this reduces the temperature slightly, so the $X M M-N e w t o n$ background is raised by $2.4 \%$ during upper limit derivations. Point sources in the XMM-Newton mosaic account for only $\sim 1 \%$ of the emission and have a spectrum that as a whole does not vary significantly from a $\Gamma \sim 2$ power law (Wik et al. 2009), so we do not model their contribution to the XMM-Newton spectrum. While their flux may artificially enhance a nonthermal signal, ignoring them will only result in slightly more conservative upper limits. For the BAT survey data, the background is automatically removed as part of the image reconstruction procedure, and systematic variations are encoded as fluctuations in empty sky regions, which is already included in the error budget.

The more significant systematic uncertainty is in the determination of the crosscalibration between Swift and XMM-Newton. Ideally, there should be no uncertainty since we based the BAT calibration on the XMM-Newton data and the Suzaku XIS0 Crab spectrum. However, the slope of the calibration (i.e. the assumed photon index of the Crab) is less certain. The total error, statistical and systematic, of the photon index in XMM-Newton EPIC Crab fits is \pm 0.05 (Kirsch et al. 2005), so we adjust our calibration to make the canonical Crab spectrum flatter by 0.05 , which acts to increase the BAT fluxes ( 10\% for E1, 20\% for E8) and flatten the BAT spectra, thus enhancing nonthermal fluxes. This approach to the systematic uncertainty is also conservative, as it is known that the Crab spectrum steepens above $10 \mathrm{keV}$ (e.g., Kirsch et al. 2005).

Since we have no clear detection of nonthermal emission, we must decide on its photon index from other arguments. The natural choice is to use the spectral index of 
the radio halo, or $\Gamma=1.5-2.5$ (Giovannini et al. 1993), though the lower range of the X-ray regime explored here corresponds to lower energy electrons where the emission may have a flatter spectrum. Also, both previous detections using RXTE and Beppo$S A X$ data found $\Gamma \sim 2$, and the model of Kushnir \& Waxman (2010) predicts this photon index. Therefore, we fix the nonthermal power law index to $\Gamma=2$, primarily because we are most interested in directly comparing our upper limits with these previous detections and model predictions. If the spectrum of nonthermal emission is in fact flatter, our upper limits will be low by some amount since the BAT errors are large and the $X M M-N e w t o n$ data will have less leverage on the fits. However, the high energy flux will not increase dramatically; as illustrated in Table 3 of Wik et al. (2009), the $20-80 \mathrm{keV}$ flux rises by a factor of 2 from $\Gamma=2$ to $\Gamma=1.5$, and trials show the same behavior for the nonthermal component in this work.

For the above systematic uncertainties, we find the $90 \%$ confidence upper limits to nonthermal emission for both the nonthermal-only BAT and for the joint EPICpn/BAT spectra. The thermal component in the latter case is simultaneously fit with the normalization of the nonthermal component. We present each individual limit in Figure 4.8 along with the vignetting corrected fluxes/upper limit from $R X T E$ (Rephaeli \& Gruber 2002, upper cross, green), Beppo-SAX (Fusco-Femiano et al. 2004, lower cross, red), and Suzaku (Wik et al. 2009, upper limit, blue). The collimator responses for these instruments are convolved with the model flux distributions to give these values or limits. The Suzaku HXD-PIN instrument is a square collimator with spatial sensitivity of the form given in Equation 3 of (Wik et al. 2009), and the $R X T E$ PCA/HEXTE and Beppo-SAX PDS instruments are hexagonal collimators with triangular approximation FWHM of $1^{\circ}$ and 1:3, respectively. We approximate 
the nearly axisymmetric response with a 4th-order polynomial of the form:

$$
R_{\mathrm{hex}}(\theta)=1.00-1.36\left(\frac{\theta}{\theta_{\max }}\right)+0.46\left(\frac{\theta}{\theta_{\max }}\right)^{2}-0.58\left(\frac{\theta}{\theta_{\max }}\right)^{3}+0.48\left(\frac{\theta}{\theta_{\max }}\right)^{4}
$$

where $R_{\text {hex }}(\theta)$ is the fraction of emission visible to the instrument at off-axis angle $\theta$ and $\theta_{\max }$ is where emission is no longer detected. For each spatial model, our upper limits are ordered in Galactic coordinates from the lowest values of $l$ and $b$ in our grid, incrementing $l$ for all positions with that latitude before incrementing $b$, with $l$ reset to the minimum value. It is this ordering that produces the pattern evident in the limits. The limits for the joint spectral fits are given in the top panel, while the nonthermal-only spectral limits are provided in the bottom panel.

Surprisingly, the upper limits derived from extended spatial models and the BAT data alone (bottom panel) are comparable in sensitivity to the previous detections/limit. Larger models are generally less constrained, due to the greater uncertainty in their estimated flux (Equation D.3), though local fluctuations have a greater impact on smaller models, increasing the spread with position. Stronger constraints are obtained when lower energy emission is simultaneously considered (top panel), and a similar range in upper limits is found for each spatial model distribution. This result is not surprising, as each model contributes roughly the same amount of flux inside the $X M M$-Newton extraction region, since most of them are extended beyond this region. Interestingly, the $\mathrm{KW}$ model provides the limits most consistent with the Beppo-SAX detection, which follows from the large PDS FOV - it would observe a higher proportion of the brighter ring emission - and the larger errors resulting from the KW model's size. However, in all realizations of the joint fit case, our $90 \%$ limits lie below the $90 \%$ interval of the previous detections/limit. Thus, all of the previous detections are excluded for any of the spatial models when one fully accounts for 


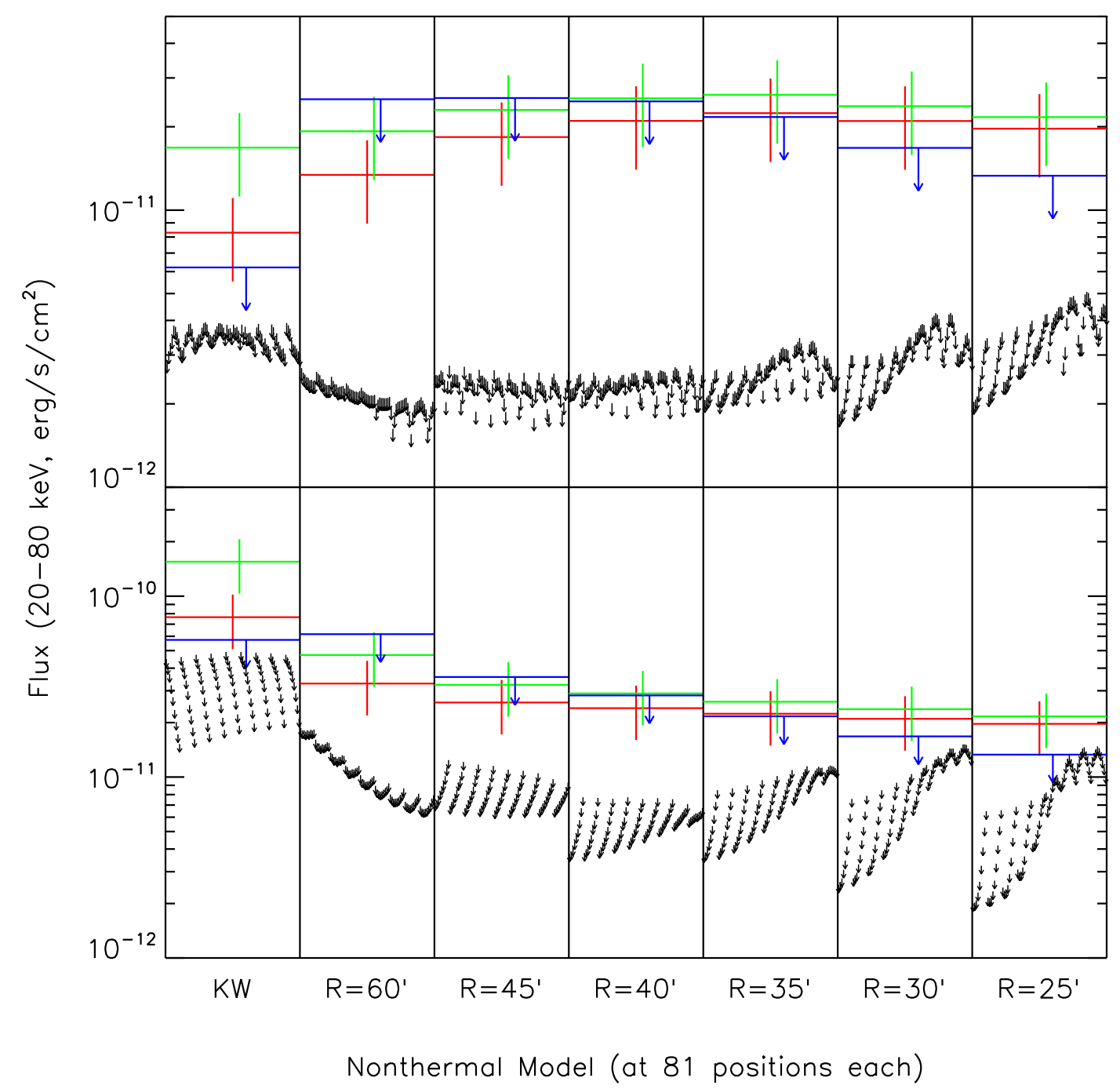

Fig. 4.8. - Upper limits (small arrows) for each nonthermal spatial model relative to the RXTE (Rephaeli \& Gruber 2002, upper cross, green), Beppo-SAX (FuscoFemiano et al. 2004, lower cross, red), and Suzaku (Wik et al. 2009, long arrow, blue) detections/upper limit. The previous flux detections $\left[(1.5 \pm 0.5) \times 10^{-11} \mathrm{ergs} / \mathrm{cm}^{2} / \mathrm{s}\right]$ and upper limit $\left(6 \times 10^{-12} \mathrm{ergs} / \mathrm{cm}^{2} / \mathrm{s}\right)$ are corrected to account for the fraction of emission missed due to vignetting by the collimator response functions (see text). In the top panel, upper limits are calculated from the simultaneous joint fits to the $X M M-N e w t o n$ and Swift spectra, and all nonthermal fluxes reported are from inside the $X M M$-Newton extraction region (the square in Fig. 4.1). In the bottom panel, upper limits are derived from BAT spectra created from the nonthermal component of spatial fits only, and the fluxes represent the total emission of the spatial model. Based on the results presented in the top panel, we conclude that extended IC emission cannot reconcile the discrepancy between the Suzaku and RXTE/Beppo-SAX observations. 
the differences in spatial sensitivity between instruments. Our upper limits, for the nominal case where the nonthermal distribution is centered on the large-scale thermal emission, are compared to these previous measurements in Table 4.2.

\subsection{Implications and Discussion}

By taking advantage of the crude imaging capabilities of the Swift BAT instrument and the impressive sensitivity of the 58-month all sky survey, we are able to constrain the amount of nonthermal, hard X-ray emission - extended or otherwise from the Coma cluster. We find no evidence for an extended, hard excess that could reconcile recent detections from $R X T E$ (Rephaeli \& Gruber 2002) and Beppo-SAX (Fusco-Femiano et al. 2004) with the upper limit from Suzaku (Wik et al. 2009); note, however, these detections would still be in conflict with the upper limit of Rossetti \& Molendi (2004). Generic, uniform surface brightness disks, along with a recently proposed IC model (Kushnir \& Waxman 2010), were fit to BAT survey images, converted to spectra, and investigated for signs of a nonthermal component. For each spatial model, we compute upper limits on a grid of positions and compare them to previous measurements, being careful to convert detected fluxes into intrinsic source fluxes, given a particular spatial distribution, by accounting for the collimator vignetting functions. These are direct comparisons, in the sense that the instrumental response of all detectors involved have been fully considered, and as such we, like Rossetti \& Molendi (2004), cannot confirm the claimed detections of Rephaeli \& Gruber (2002) and Fusco-Femiano et al. (2004).

Their observed hard excesses could have had other reasonable sources, if not diffuse IC emission from the nonthermal phase of the ICM. A common difficulty is an accurate determination of both the cosmic and non-X-ray background, the treatment of which 
is the primary difference between Fusco-Femiano et al. (2004) and Rossetti \& Molendi (2004). Another possibility is the variable nature of nearby point sources, most notably the AGN W Comae, which was once quite bright but has been fading for many years. The concurrence of the $R X T E$ and Beppo-SAX observations could have simply been unlucky and caught W Comae (or another source) in a bright state. A somewhat more subtle, and perhaps more likely, explanation concerns the multitemperature nature of Coma's ICM. Small amounts of hot gas could dominate the high energy emission, so the extrapolation of an average temperature determined from lower energy data may not be an adequate description of the thermal contribution to the high energy flux. The effect of the multi-temperature gas in Coma is evident in the SW extension at hard energies observed by INTEGRAL (Renaud et al. 2006b; Eckert et al. 2007a) and confirmed here; higher temperatures seen at this location in the temperature map (Wik et al. 2009) are sufficient to explain the change in morphology, which points to the increased significance of this gas at higher energies. Even so, single temperature spectral fits do not produce IC detections in this study or in Wik et al. (2009). A more likely explanation may simply rest in a slight mischaracterization of the hard energy emission weighted temperature; in Fusco-Femiano et al. (2004), the FOV of the lower energy HPGSPC instrument does not quite match the higher energy PDS, and the temperature of $7.67 \pm 0.1 \mathrm{keV}$ found in Rephaeli \& Gruber (2002) is significantly below that allowed by the XMM-Newton data.

Given radio synchrotron emission and an upper limit on the X-ray IC flux, a lower limit on the average ICM magnetic field can be estimated, as described by equation (13) in Wik et al. (2009) and the accompanying text. A diffuse radio flux of $640 \mathrm{mJy}$ at $1.4 \mathrm{GHz}$ is detected out to a radius of $\sim 40^{\prime}$ in Deiss et al. (1997). For comparison, we will use the upper limit of $2.7 \times 10^{-12} \mathrm{erg} \mathrm{s}^{-1} \mathrm{~cm}^{-2}(20<E<80$ 
$\mathrm{keV}$ ) from the $R=40^{\prime}$ disk model. These values imply $B>0.25 \mu \mathrm{G}$, an increase from Wik et al. (2009) but still well below the equipartition value of $B_{\text {eq }}=0.5 \mu \mathrm{G}$ for the Coma radio halo (Giovannini et al. 1993). A slightly lower limit of $B>0.2 \mu \mathrm{G}$ results if a more conservative IC upper limit of $4.2 \times 10^{-12} \mathrm{erg} \mathrm{s}^{-1} \mathrm{~cm}^{-2}$ is used, which considers the limits from all spatial models tested. Regardless, these limits on $B$ fall well below line of sight estimates of several $\mu \mathrm{G}$ from Faraday rotation measure (RM) observations (Feretti et al. 1995), though due to geometric effects these measurements may not represent the average cluster magnetic field (Petrosian 2001).

However, the global field may be recovered by combining many RM measurements along different lines-of-sight through the ICM with numerical simulations (Murgia et al. 2004). Bonafede et al. (2010) have applied this method to the Coma cluster, deriving a radial profile where the energy density of the magnetic field falls roughly in proportion with the energy density of thermal gas and with a central field strength of $B_{0} \sim 4.7 \mu \mathrm{G}$. Combining this model of $B(r)$ with an approximate representation of the radial density profile of synchrotron emission, implied by a rough $\beta$-model fit to the point source subtracted image of Deiss et al. (1997) $\left(r_{c}=18^{\prime}, \beta=1\right.$, and $I_{0}=1.23 \mathrm{mJy} \operatorname{arcmin}^{-2}$ ), directly leads to a prediction of the expected IC surface brightness as a function of radius. Our illustrative - due to the large uncertainties in all parameters assumed in this exercise - IC surface brightness distribution is flat out to $\sim 30^{\prime}$ with a 20-80 keV flux of $\sim 8 \times 10^{-17} \mathrm{erg} \mathrm{s}^{-1} \mathrm{~cm}^{-2} \operatorname{arcmin}^{-2}$, at which point it nearly linearly drops toward zero, though not reaching it, around a radius of $90^{\prime}$. This surface brightness is about an order of magnitude below that implied by our upper limits, providing a possible explanation for why we are unable to detect an IC signature. On the other hand, larger IC fluxes would be expected if the radio synchrotron emission falls off more gradually than modeled here, since 
a flatter radial profile would suggest a higher relativistic electron density given the falling magnetic field with cluster radius. More accurate maps of Coma's radio halo, preferably at lower frequencies where the radio electrons correspond more closely to the IC-emitting electrons, will clarify this issue.

Ultimately, a true detection of IC emission from Coma will have to wait for upcoming missions with focussing hard X-ray telescopes, namely $N u S T A R^{2}$ and Astro- $H^{3}$. For $N u S T A R$ to achieve a sensitivity comparable to our upper limits, a single pointed observation of at least $100 \mathrm{ks}$ will be required (Madsen et al. 2009). However, the much finer spatial resolution will remove the uncertainty associated with bright background AGN and allow multiple spatially-resolved joint fits. Assuming the hottest gas, which produces the largest amount of thermal emission at hard energies, is localized, then these regions can be identified and avoided in order to detect a lower surface brightness, but more uniform, IC component. Similarly, if the IC emission is more localized, it will be easier to identify with spatially-resolved joint fits between XMM-Newton and NuSTAR or Astro-H spectra, as has been done with Chandra data alone (Million \& Allen 2009). The unambiguous detection of IC emission associated with radio halos and relics is crucial to determining the energy content in the relativistic phase of the ICM and how significant of an influence this phase has on the dynamics and structure of the thermal gas in clusters.

\footnotetext{
${ }^{2}$ http://www.nustar.caltech.edu/

${ }^{3}$ http://astro-h.isas.jaxa.jp/
} 
Table 4.2. $90 \%$ Flux Upper Limits (20-80 keV) for Nominal NT Position ${ }^{\mathrm{a}}$

\begin{tabular}{lcrrr}
\hline \hline Model & $\begin{array}{c}\text { Joint BAT limit } \\
10^{-12} \operatorname{cgs}\end{array}$ & $\begin{array}{c}\text { Beppo-SAX } \\
10^{-12} \mathrm{cgs}\end{array}$ & $\begin{array}{c}\text { RXTE } \\
10^{-12} \mathrm{cgs}\end{array}$ & $\begin{array}{c}\text { Suzaku } \\
10^{-12} \mathrm{cgs}\end{array}$ \\
\hline $\mathrm{KW}$ & $<3.86$ & $16.8 \pm 5.6$ & $8.3 \pm 2.8$ & $<6.2$ \\
$\mathrm{R} 60$ & $<2.16$ & $19.3 \pm 6.4$ & $13.4 \pm 4.5$ & $<25.1$ \\
$\mathrm{R} 45$ & $<2.34$ & $23.0 \pm 7.7$ & $18.4 \pm 6.1$ & $<25.4$ \\
$\mathrm{R} 40$ & $<2.53$ & $25.3 \pm 8.4$ & $21.0 \pm 7.0$ & $<24.7$ \\
$\mathrm{R} 35$ & $<2.95$ & $26.1 \pm 8.7$ & $22.4 \pm 7.5$ & $<21.7$ \\
$\mathrm{R} 30$ & $<3.22$ & $23.7 \pm 7.9$ & $21.0 \pm 7.0$ & $<16.8$ \\
$\mathrm{R} 25$ & $<3.48$ & $21.7 \pm 7.2$ & $19.7 \pm 6.6$ & $<13.3$ \\
\hline
\end{tabular}

${ }^{\mathrm{a}}$ Units for flux are $10^{-12} \mathrm{erg} \mathrm{cm}^{-2} \mathrm{~s}^{-1}$ 


\section{Chapter 5}

\section{The Swift BAT Perspective on Nonthermal Emission in HIFLUGCS Galaxy Clusters ${ }^{1}$}

\section{Abstract}

The search for diffuse nonthermal, inverse Compton (IC) emission from galaxy clusters at hard X-ray energies has been underway for many years, with most detections being either of low significance or controversial. Until recently, comprehensive surveys of hard X-ray emission from clusters were not possible; instead, individually proposedfor, long observations would be collated from the archive. With the advent of the Swift BAT all sky survey, any cluster's emission above $14 \mathrm{keV}$ can be probed with nearly uniform sensitivity, which with the 58-month version of the survey is comparable to that of $R X T E$, Beppo-SAX, and Suzaku. In this work, we search for nonthermal excess emission above the exponentially decreasing, high energy thermal emission in the flux-

\footnotetext{
${ }^{1}$ Nearly ready for submission to the Astrophysical Journal
} 
limited HIFLUGCS sample. The BAT emission from many of the detected clusters is marginally extended; we are able to extract the total flux for these clusters following the procedure detailed in Chapter 4. To account for thermal emission at BAT energies, $X M M$-Newton EPIC spectra are extracted from coincident spatial regions so that both the thermal and nonthermal spectral components can be determined simultaneously in joint fits. We find marginally significant IC components in 6 clusters, though after closer inspection and consideration of systematic errors we are unable to claim a detection in any of them. The spectra of all clusters are also summed to enhance a cumulative nonthermal signal not quite detectable in individual clusters. After constructing a model based on single temperature fits to the XMM-Newton data alone, we see no significant excess emission above that predicted by the thermal model determined at soft energies. This result also holds for the summed spectra of various subgroups, except for the subsample of clusters with diffuse radio emission. For clusters hosting a radio halo or relic, nonthermal emission is initially detected at the $\sim 3 \sigma$ confidence level, but the inclusion of systematic uncertainties undermines its significance. Ultimately, though our samples are not identical, we find that our BAT spectra generally agree with the Nevalainen et al. (2004) spectra from the Beppo-SAX PDS instrument.

\section{$5.1 \quad$ Introduction}

A number of observations, mainly at radio frequencies, have established that relativistic particles and magnetic fields are part of the intracluster medium (ICM) of galaxy clusters (e.g., Govoni \& Feretti 2004). The large ( Mpc) scale, diffuse structures known as radio halos and relics are produced by relativistic electrons spiraling around $\sim \mu \mathrm{G}$ magnetic fields. Because halos and relics are not detected in every clus- 
ter, but are preferentially found in clusters with ongoing major merger activity (Buote 2001; Schuecker et al. 2001), mergers probably temporarily reaccelerate underlying relativistic populations (e.g., Sarazin 1999; Brunetti \& Blasi 2005). It is important to fully characterize the nonthermal phase if the dynamics and general state of the ICM is to be understood; the proportion of energy tied up in these relativistic components, if significant, may bias inferred mass estimates necessary to use clusters as cosmological probes (e.g., Mantz et al. 2008; Vikhlinin et al. 2009; Vazza et al. 2009). Unfortunately, synchrotron emission alone cannot separately determine particle and magnetic field energy densities, and so the total energy in the nonthermal phase remains relatively unconstrained. However, the electron population can be independently observed through inverse Compton (IC) emission due to scattering of the ubiquitous Cosmic Microwave Background (CMB) photons, which are up-scattered to X-ray energies and may be observable if the electron population is sufficiently large (Rephaeli 1979). Detections of IC emission, therefore, have the potential to determine whether the nonthermal phase is energetically negligible or, particularly if the average magnetic field is large, it is sizable enough to affect the dynamics and structure of the thermal gas.

Thermal emission clearly dominates at $\sim \mathrm{keV}$ energies, so searches for excess emission due to an IC spectral component must be undertaken at very soft or hard (> $10 \mathrm{keV}$ ) energies. The latter range is particularly promising, given the exponential decline in the thermal spectrum and the lack of Galactic and solar wind charge exchange foregrounds that can hamper searches at soft energies (Koutroumpa et al. 2009; Takei et al. 2007; Bonamente et al. 2007). In particular, the Swift BAT all sky survey (Tueller et al. 2010) provides a deep map of hard energy (14-195 keV) emission from which nonthermal excesses can be identified. Its uniform coverage and impres- 
sive sensitivity makes it the most complete dataset from which to study the brightest objects in a given class (e.g., Winter et al. 2009). Whereas previous searches have concentrated on long pointed observations of individual clusters, this survey allows a larger, more uniform sample to be searched, as already undertaken with an earlier epoch of the survey (Ajello et al. 2009). To take full advantage of this capability, we have chosen the flux-limited HIFLUGCS sample (Reiprich \& Böhringer 2002), which contains the brightest clusters in the sky outside the Galactic plane. A selection of the brightest clusters provides the greatest opportunity to detect IC emission, as the IC signal could reasonably be thought to be similarly bright. Also, because these clusters are bright and contained within a well-defined survey, there already exist good observations at lower X-ray energies, which can be used to strongly constrain the thermal properties of the ICM - an important prerequisite for the robust detection of an IC excess. Finally, the fact that HIFLUGCS is a complete flux-limited allows one to discuss the statistic properties of their hard excesses by stacking the individual cluster observations with the Swift BAT.

Because they are nearby and bright, many of the clusters in HIFLUGCS have been targets of IC searches with other telescopes, including A3667 (Finoguenov et al. 2010), A3112 (Bonamente et al. 2009), A3376 (Kawano et al. 2009), A2256 (FuscoFemiano et al. 2005), A1367 (Henriksen \& Mushotzky 2001), A2199 (Kempner \& Sarazin 2000), and A2163 (Rephaeli et al. 2006). Most often clusters are targeted because they host a radio halo or relic, and the IC flux leads to a direct measure of the average magnetic field strength. A large fraction of HIFLUGCS clusters were also included in an analysis of all long exposure Beppo-SAX observations (Nevalainen et al. 2004), which found marginal evidence for nonthermal excesses in individual clusters but a substantial excess in a stacked spectrum. In general, an IC component 
distinct from thermal emission in the hard band has been difficult to clearly identify, with perhaps the only counter example being an exceptionally deep observation of the Ophiuchus cluster (Eckert et al. 2008). Perhaps the cluster most thoroughly searched for nonthermal emission, also in HIFLUGCS, is the Coma cluster. Controversial (Rossetti \& Molendi 2004) detections with RXTE (Rephaeli \& Gruber 2002) and Beppo-SAX (Fusco-Femiano et al. 2004) have recently been excluded with comparable Suzaku (Wik et al. 2009) observations and a detailed analysis of the Swift BAT survey data (Chapter 4).

To perform the deepest hard X-ray survey of nonthermal emission in clusters to date, we jointly fit high quality XMM-Newton EPIC and Swift BAT spectra, extracted from identical regions and cross-calibrated to make their absolute spectral responses as similar as possible. We describe the data and its calibration in Section 5.2. In Section 5.3, the thermal and nonthermal character of the spectra are separately analyzed, and in Section 5.4 they are jointly fit for each individual cluster. We also search for a statistic hard excess excess in sets of stacked spectra for the entire sample and for several subsamples in Section 5.5. Lastly, the implications of our results are discussed in Section 5.6. We assume a flat cosmology with $\Omega_{M}=0.23$ and $H_{0}=72 \mathrm{~km} \mathrm{~s}^{-1}$ $\mathrm{Mpc}^{-1}$. Unless otherwise stated, all uncertainties are given at the $90 \%$ confidence level.

\subsection{Observations and Data Preparation}

\subsubsection{XMM-Newton EPIC Spectra}

For the lower energy BAT bands, it is very useful to have X-ray spectra at lower energies to constrain the thermal emission; this is particularly true given that the 
SwiftBAT survey spectra are coarsely binned (8 channels spanning $14 \mathrm{keV}<E<195$ $\mathrm{keV}$ ). Also, any nonthermal component in the BAT spectra must be consistent with the spectra at softer energies. XMM-Newton is the ideal observatory to provide such complementary spectra. For one, its large field of view (FOV) allows a higher fraction of the total emission, which can be quite extended given the low redshift of the sample, to be detected in a single pointing. Additionally, the EPIC instruments are sensitive to 5-10 keV photons, which make them more useful for constraining the highest temperature gas, and the telescopes have good spatial resolution so that point sources can be excluded from the spectra. Last, but of no less importance, XMM-Newton has observed all but one (Abell 2244) of the clusters in HIFLUGCS. Unfortunately, another 4 cluster observations (Abell 401, Abell 478, Abell 1736, and Abell 2163) are heavily contaminated by background flares and consequently unusable. However, the data for the remaining 58 clusters are of sufficient quality to help constrain potential nonthermal signals in the BAT energy bands.

We extract $X M M$-Newton spectra for each cluster from the largest circular region that either covers the FOV or extends to the point where cosmic X-ray background (CXB) emission begins to dominate. To ensure near Gaussian statistics for $\chi^{2}$ fitting, adjacent channels are grouped until each new bin contains at least 30 counts. The centers and radii of the circular regions, along with each pointing's observation ID, are listed in Table 5.1. Source spectra are extracted in concentric annuli within the region; corresponding particle background spectra are derived from CLOSED mode calibration data, which are renormalized based on $E>12 \mathrm{keV}$ events, where this background is dominant (for details see Zhang et al. 2006). The full background treatment is described in Zhang et al. (2009). As an additional step, we readjust the normalization of the particle background spectra by hand to ensure the $7-12 \mathrm{keV}$ con- 
tinuum of the cluster spectra have a more physical shape. We define "more physical" as the background normalization that minimizes the $\chi^{2}$ statistic for a single temperature (1T) (using the APEC plasma emission model ${ }^{2}$ ) individually fit to the EPIC-pn, MOS1, and MOS2 spectra, from $2<E<12 \mathrm{keV}$. The new best-fit temperatures, after these initial renormalizations of the background, are compared to each other and to previous measurements (primarily Reiprich \& Böhringer 2002). While this method may bias the background level, especially if a single temperature model is a poor description of a given spectrum, repeating this procedure with two temperature $(2 \mathrm{~T})$ and single temperature plus power law $(\mathrm{T}+\mathrm{NT})$ models yield comparable or inferior results, usually favoring obvious under-subtractions of the background that produce systematic patterns in the residuals. We favor normalizations that leave the background slightly under-subtracted, in order to avoid removing a real nonthermal signature. For the most part, the overall spectrum is only mildly affected since much of the emission is at lower energies where the background is a smaller fraction of the total. One consequence is that instrumental lines, which are typically between 7.5 and $9.5 \mathrm{keV}$ and mainly are a problem in the EPIC-pn spectra and which can vary in intensity relative to the background continuum, can be under- or over-subtracted. No resolved ICM lines exist in this range, so we simply ignore this region when poor line subtractions occur, as in Wik et al. (2009). Based on the change in $\chi^{2}$ as the background normalization is varied, a typical $90 \%$ level uncertainty in the normalization is $\sim 3 \%$.

We choose to model, instead of subtract, one further background component: the CXB due to extragalactic sources. Lumb et al. (2002), using XMM-Newton sky fields, find that this component of the CXB is well fit by a power law with photon index of 1.42 in the hard band $(2-10 \mathrm{keV})$. Their results are in good agreement with other

\footnotetext{
${ }^{2}$ http://cxc.harvard.edu/atomdb/sources_apec.html
} 
work in this band (e.g., Moretti et al. 2003; De Luca \& Molendi 2004). We adopt their normalization at $1 \mathrm{keV}$ of 8.44 photons $\mathrm{cm}^{-2} \mathrm{~s}^{-1} \mathrm{keV}^{-1} \mathrm{sr}^{-1}$, which is scaled to match the extraction area for each cluster. The impact of cosmic variance, or the field-to-field variation in CXB flux resulting from large scale structure and source population selection, is not included as a systematic uncertainty in the following analysis due to its small effect. While cosmic variance increases with decreasing solid angle, the high sensitivity of XMM-Newton allows most of the sources responsible for a higher variance to be removed, so for one of our typical regions the $90 \%$ uncertainty is only $\sim 10 \%$ of the CXB flux. Note that Lumb et al. (2002) remove detected point sources as is done here, so their spectrum can be directly applied as is. The Galactic component of the CXB is also not considered, as it only contributes below $1 \mathrm{keV}$.

\subsubsection{Swift BAT 58-month Survey Spectra}

The Swift mission and the properties of the survey are described in detail in Section 4.2.2 and in Tueller et al. (2010). Similarly, we refer to that Section and the appendices for details on the extraction and calibration of sources from survey image data. To briefly summarize, the flux calibration is tied to the Crab spectrum, which we define to have the same spectrum as that observed by XMM-Newton for $E>2$ $\mathrm{keV}$, extrapolated to BAT energies. In this way, both the cross-normalization and spectral shape of the XMM-Newton and Swift spectra will match, and continuous models can be jointly fit to them simultaneously.

While the standard processing of coded mask imaging data is designed to extract the fluxes of point sources, it is also possible to extract the flux of a mildly extended source, albeit with somewhat greater uncertainty [Renaud et al. (2006b), Chapter 4]. The large effective PSF $\left(\sim 20^{\prime}\right.$ full width at half maximum $\left.[\mathrm{FWHM}]\right)$ for point sources 
in the survey means that even clusters of galaxies will appear only slightly extended; the FWHM of the Coma cluster - the most extended detected source in the survey - is only 28:5. From Figure 5.1, it is clear that detected clusters (in the 14-20 $\mathrm{keV}$ band) are typically extended, relative to other sources. The filled (red) circles represent the average source FWHM (excluding clusters) within a radius of $30^{\circ}$ for 18 independent regions, showing that the survey PSF is fairly constant over the entire sky. The hatched region encloses one standard deviation, or $68 \%$, of the non-cluster source FWHM. Individual cluster FWHM (triangles, green) are systematically more extended; detections with $\mathrm{S} / \mathrm{N}>10$ are circled in blue. We follow the procedure outlined in Chapter 4 to extract fluxes for diffuse sources, which requires the spatial distribution of the emission to be known. Because clusters are comparable in size to the effective spatial resolution of the survey, detailed spatial models are not necessary to extract accurate fluxes. We consider generic $\beta$-model surface brightness profiles, which well represent the radial profiles at softer energies. Taking a representative value for $\beta$ of 0.75 , we find that all $>3 \sigma$ detected clusters (in a given band) can be well fit with core radii $r_{c}$ of either $4^{\prime}, 6^{\prime}, 8^{\prime}$, or $10^{\prime}$. Profiles with $r_{c}<4^{\prime}$ are hard to distinguish from point source profiles, so for any cluster emission that is too narrow to be fit with the $r_{c}=4^{\prime}$ model is treated as a point source. The true spatial distribution may differ from these fiducial models, but our aim is only to extract accurate fluxes, not describe the distribution of hard X-ray emission. For Coma, a $\beta$-model fit in the first BAT band (E1: 14-20 keV) yields a total flux 9\% lower than that derived from a more detailed model of its spatial distribution derived from an XMM-Newton temperature map (see Chapter 4), which accounts for the NE-SW non-axisymmetric elongation of the emission (Eckert et al. 2007a). While $9 \%$ is a significant difference, Coma is one of the most significantly detected and is the most extended cluster in the 
survey, so this deviation, which amounts to a factor of only 1.6 times the $1-\sigma$ error on the flux, is the largest we would expect using this set of extended models.

We also investigated the use of diffuse models for all the clusters, irrespective of their observed extent, to account for the possibility that we are missing low surface brightness emission obscured by noise. Since the spatial distribution of $E>10$ $\mathrm{keV}$ emission is unknown, we assume $\beta$-model profiles derived from ROSAT images (Reiprich \& Böhringer 2002). For clusters with a clearly extended BAT profile, these models reasonably, but usually not perfectly, follow the emission. Spectral fits using these fluxes produce similar results to those we present in this work, but because their associated errors are larger, these spectra are generally less sensitive, so any additional flux captured - which is not significant -is also diluted. Therefore, these spectra are not considered further.

For clusters with modeled extended emission, we do not want to include the portion of flux that falls outside the $X M M$-Newton extraction region during joint fits of the data, since the complementary softer flux in the $X M M-N e w t o n$ band spectra is not present. Therefore, only the fraction of the flux that resides within the XMM-Newton region is included in the spectra derived here. One uncertainty, particularly when emission is detected at lower significance, is where the emission is actually coming from, given the positional accuracy of the survey (a $5 \sigma$ source detected in a given band has a $90 \%$ error circle of radius $6^{\prime}$ ). Since the E1 band-derived positions are near the center of the extraction region, within their respective error circles, we assume the center of the hard band distribution is coincident with the center of the $X M M-N e w t o n$ extraction region. This way we will not underestimate the coincident flux, although overestimates may result that could lead to incorrect hard excesses. However, since we are unable to significantly detect nonthermal emission individually 


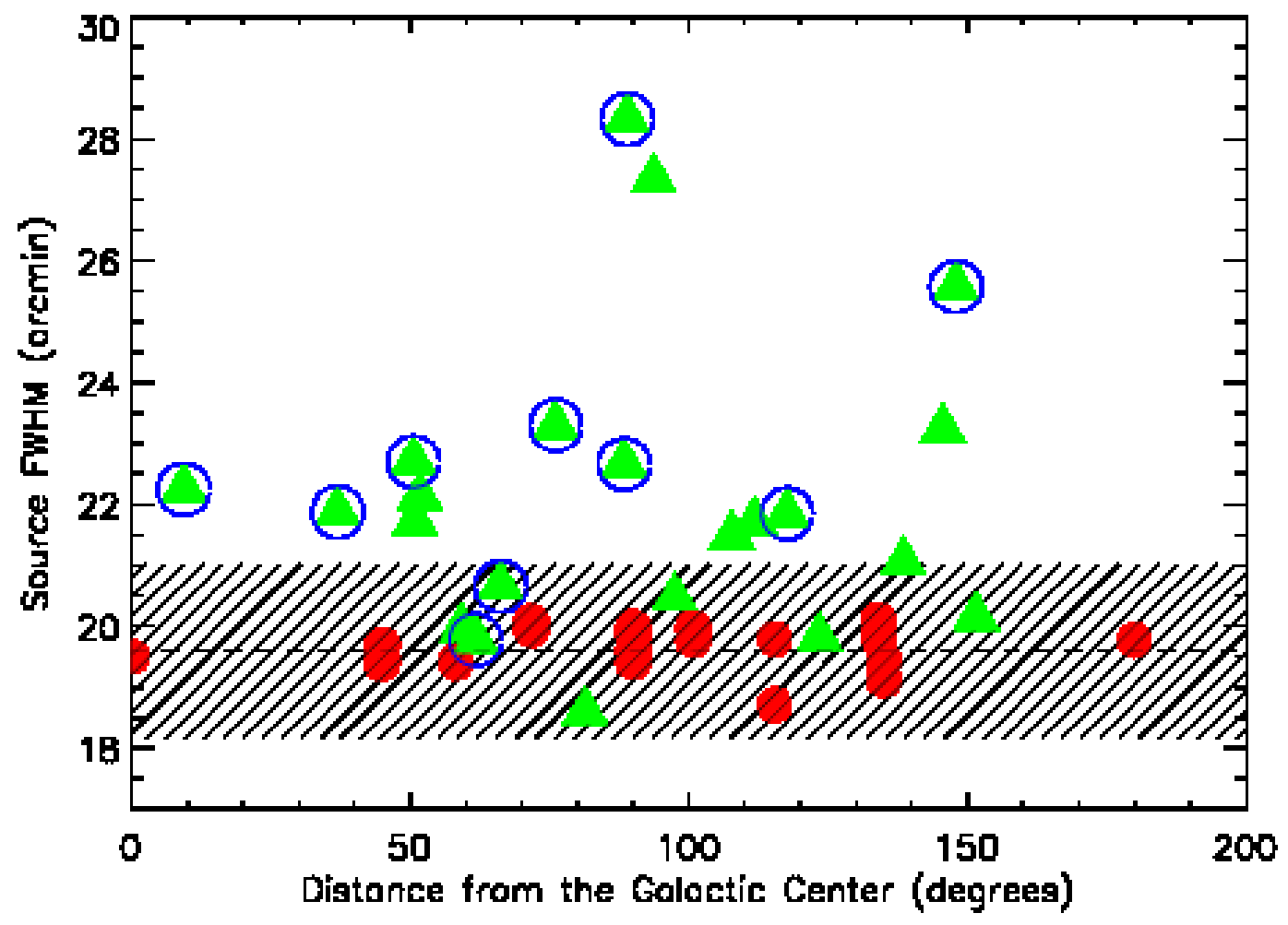

Fig. 5.1. - Source extent as a function of distance from the Galactic center in the 14$20 \mathrm{keV}$ BAT band. Filled circles (red) represent the mean FWHM of all non-cluster sources within a region of $30^{\circ}$ radius for various independent positions distributed evenly over the sky. The hatched area marks one standard deviation of the FWHM distribution for all non-cluster sources. Note that the red circles indicate the average FWHM of several sources; consequently, their scatter about the mean FWHM value of all sources, indicated with the dashed line, will be reduced by the square root of the number of objects inside that region, i.e. the "error of the mean," which is why their scatter is less than that shown by the hatched area. Detected clusters are shown as triangles (green) and are circled if they have $\mathrm{S} / \mathrm{N}>10$. Many bright galaxy clusters are at least somewhat resolved by the BAT, which must be accounted for when extracting fluxes from the BAT survey. 
in any of the clusters, this procedure can only cause us to be biased in favor of more conservative upper limits.

\subsection{Separate Fits to Individual $X M M-N e w t o n$ EPIC and Swift BAT Spectra}

Before combining the Swift and XMM-Newton datasets, we characterize each telescope's spectra separately. The goal is to identify any problems with the data or our methodology that might lead to biased results when the spectra are fit jointly.

\subsubsection{Single Temperature Fits to the EPIC Spectra}

The motivation for including $X M M-N e w t o n$ spectra in the analysis is to fully characterize the thermal properties of the hottest gas in the ICM, which will contribute flux to the BAT energy bands. Similarly, these lower energy spectra must be consistent with any indication of a nonthermal component in the BAT spectra; for example, a steep power law may best describe the BAT data but at lower energies result in a poor description of the spectrum. Since our purpose is not to fully characterize the total emission detectable by XMM-Newton, but only capture the state of the hottest

gas, we ignore all events with energies below $2 \mathrm{keV}$. Cool $(\lesssim 1 \mathrm{keV})$ gas is completely unimportant at BAT energies, and it will not overly bias $E>2 \mathrm{keV}$ data. We therefore initially consider EPIC spectra in the 2-12 keV range; including photons down to $2 \mathrm{keV}$ provides additional leverage during spectral fitting, since most of the detected photons, regardless of temperature, are at lower energies.

However, the lower end of this energy range presents two issues. First, bright $\sim 1 \mathrm{keV}$ gas can significantly contribute to the emission between 2 and $3 \mathrm{keV}$, which 
certainly exists in some of the cool core clusters in HIFLUGCS. In single temperature fits, the average temperature will then be biased low to accommodate this component, which could lead to thermal emission being interpreted as a nonthermal excess. Multitemperature fits would alleviate this problem, but the $X M M-N e w t o n$ data are not of sufficient quality to strongly constrain more than one temperature component in this energy range. Including $E<2 \mathrm{keV}$ data to better constrain multi-temperature fits would also require a more complicated analysis that will involve more free parameters and, because the highest signal-to-noise ratios are in the $\sim 1 \mathrm{keV}$ channels, fits would be driven by this data, possibly resulting in biased high temperature components. The second issue relates to the imperfectly calibrated gold edge at $2.2 \mathrm{keV}$, where the response drops somewhat abruptly. While on its own this feature does not strongly impact spectral fits, because it lies near the edge of our energy range where the signal-to-noise is largest, secondary model components can be "co-opted" into better fitting this edge. For instance, in a spectrum truly described by a gas at a single temperature, the addition of a second temperature or nonthermal component to the fit will cause the second component to "fix" this edge, typically resulting in a low temperature or steep photon index that has no real physical counterpart.

In practice, both of these effects can conspire to produce the appearance of a more significant nonthermal spectral component than is warranted by the rest of the data. To counter both issues, we also perform fits to data with energies $E>3 \mathrm{keV}$, which exclude the gold edge and any sizable emission from $\lesssim 1 \mathrm{keV}$ gas. These spectra have lower signal-to-noise due to excluding the $2-3 \mathrm{keV}$ emission, but the high fluxes of clusters in our sample reduce this issue's importance. Single temperature fits in both the 2-12 keV and 3-12 keV ranges, jointly fit to all three EPIC spectra (except for A3526, for which the MOS-1 spectrum is ignored, and for A2142 and A2147, for 
which the MOS-2 spectra are ignored), are given in Table 5.2. The pn and MOS instrument cross-normalization is left as a free parameter, which allows for a typical $(10 \pm 10) \%$ difference between their calibration. This cross-normalization factor is used and kept fixed during all subsequent joint EPIC-BAT fits. The change in the best-fit temperature from the $E>2 \mathrm{keV}$ to $E>3 \mathrm{keV}$ fits is only $\sim 0.3 \mathrm{keV}$ on average, indicating that the temperature is generally robust to the choice of the energy range, but that higher energy photons come preferentially from higher temperature gas, assuming the true temperature structure is not isothermal but contains a continuous spectrum with gas at many temperatures due to substructure or radial gradients (Cavagnolo et al. 2008b; Snowden et al. 2008).

\subsubsection{Nonthermal Fits to the BAT Spectra}

Our goal is to detect a nonthermal spectral component at hard energies, but because the statistical weight of the BAT channels is so much less than the EPIC channels (lower $\mathrm{S} / \mathrm{N}$ and fewer of them, at least by an order of magnitude), we have to be careful not to let the XMM-Newton data unfairly drive the spectral fits. To assess the sensitivity of our BAT spectra, we extract 10,000 blank sky spectra from uniformly distributed, random positions at least $40^{\prime}$ from any known sources and greater than $20^{\circ}$ from the Galactic plane, to mimic the selection function in HIFLUGCS. We then fit these spectra with a fiducial power law model of photon index $\Gamma$ fixed at a value of 2 , then expected slope for IC emission inferred from radio halos and relics. The distribution of best-fit normalizations from these power law fits are presented in the black histogram in Figure 5.2. They are well fit by a symmetric Gaussian (red dashed line) and indicate a $1 \sigma$ sensitivity threshold of $\sim 2 \times 10^{-12} \mathrm{erg} \mathrm{cm}^{-2} \mathrm{~s}^{-1}(20-80 \mathrm{keV})$. Similarly, the formal $3 \sigma$ detection level is $5.8 \times 10^{-12} \mathrm{erg}^{-2} \mathrm{~s}^{-1}$. Clearly, the BAT 
is sensitive enough to confirm or reject previous IC detections with fluxes $\sim 10^{-11} \mathrm{erg}$ $\mathrm{cm}^{-2} \mathrm{~s}^{-1}$ (e.g., Rephaeli \& Gruber 2002; Fusco-Femiano et al. 2004).

Now we wish to compare our cluster spectra with this distribution, but first we have to account for any thermal emission in the lower energy bands. The single temperature models derived with XMM-Newton $(2-12 \mathrm{keV})$ are included as a second component along with the power law model, with only its normalization left as a free parameter. The resulting nonthermal normalizations are also given in Figure 5.2 in blue as both the wider histogram (scaled up) and as the vertical lines (showing individual values). While the majority of cluster nonthermal components are consistent with the blank sky fits, there is a tail at positive normalizations possibly indicative of a nonthermal excess. However, the thermal contribution is not well determined in this method and may be underestimated. Intriguingly, the three clusters with the most significant nonthermal component (A2029, A1367, and A1651) have positive fluxes, although marginally detected, in all 8 BAT bands; this rarely occurs for the blank sky spectra. We discuss these clusters in more detail later. The main result from this analysis is that the BAT cluster spectra have probably not reached a sensitivity level sufficient to detect hard, nonthermal excesses, if they exist, in the brightest clusters.

\subsection{Joint Fits to the EPIC-BAT Spectra}

BAT fluxes are calibrated to match both the normalization and the spectral shape of sources as detected by the XMM-Newton EPIC-pn instrument (Chapter 4), and they are extracted from regions identical to the XMM-Newton extraction regions. As such, continuous spectral models can be used over the full 2-195 keV energy range to simultaneously fit both the XMM-Newton and Swift spectra. However, in individual cases the cross-normalization factor, $f_{C N}$, may stray from a value of 1 


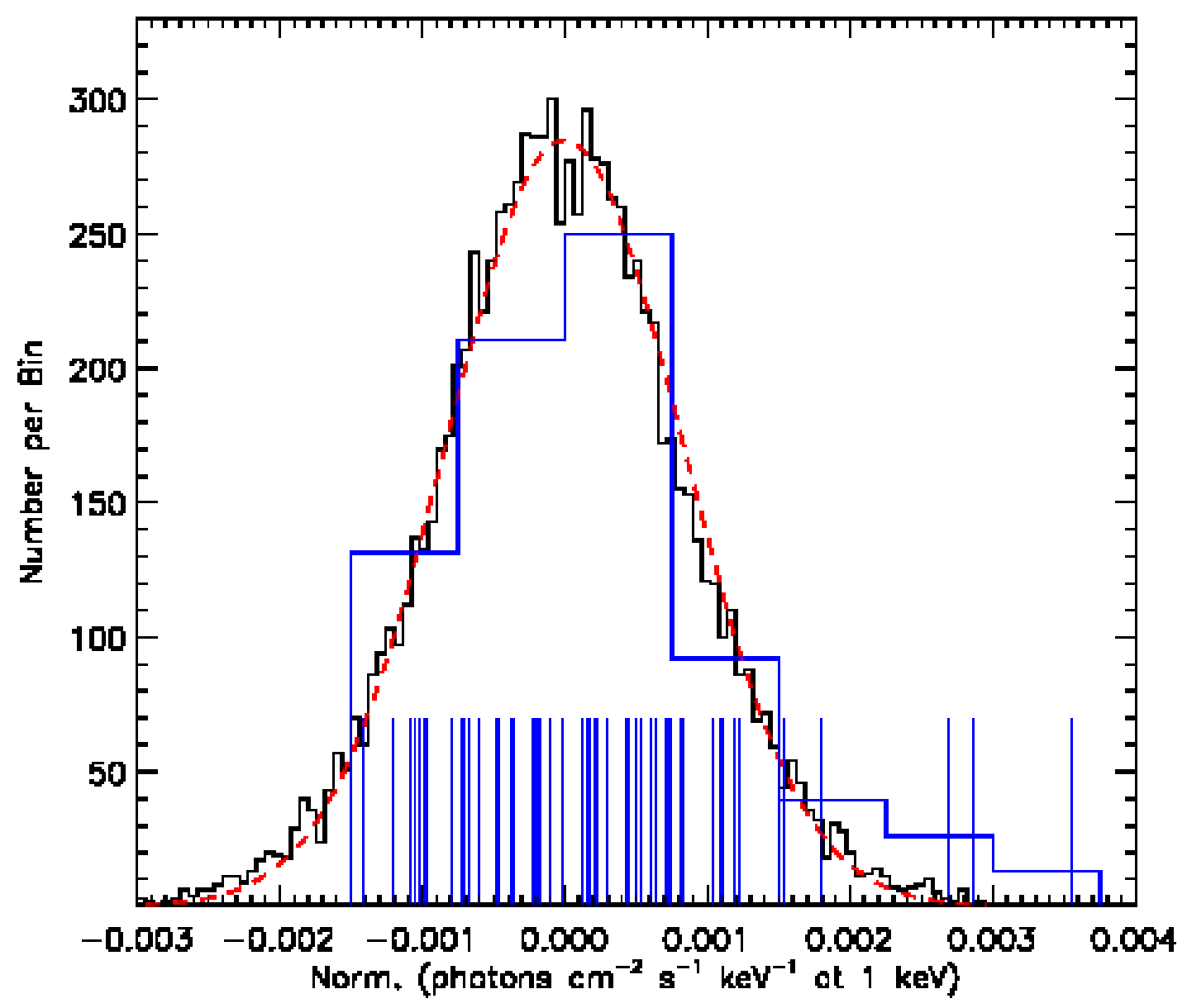

Fig. 5.2. - The distribution of power law normalizations (with a fixed photon index $\Gamma=2$ ) fit to 10,000 blank sky spectra extracted from the BAT survey (black histogram). The best-fit Gaussian distribution is overlaid as the smooth, dashed red line. In blue, similar best-fit normalizations are shown for the 58 HIFLUGCS clusters (see text for details), with individual normalizations represented as vertical lines. The cluster histogram has been scaled up to show its agreement with the blank sky spectra. In general, the cluster BAT spectra lack any clear evidence for a nonthermal component, except in a few cases comprising the positive tail of the blue histogram. 
as it does between the pn and MOS instruments (see Section 5.3.1). We therefore adopt, along with a $3 \%$ uncertainty in the XMM-Newton background normalizations, a conservative $10 \%$ systematic uncertainty for $f_{C N}$. Because no compelling evidence for nonthermal emission is found in the nominally calibrated spectra (see analysis below), we only consider these uncertainties when deriving $90 \%$ confidence interval upper limits.

\subsubsection{General Properties from the Joint Analysis}

For each cluster, 3 simple spectral models are employed to describe the emission covering 2 orders of magnitude in energy: a single temperature thermal model (1T), a two temperature model $(2 \mathrm{~T})$, and a thermal plus nonthermal model $(\mathrm{T}+\mathrm{IC})$. Due to the limited sensitivity of the Swift data, more complicated models cannot be constrained; for example, the separate temperature components in the $2 \mathrm{~T}$ model are generally unconstrained for our sample. Above $50 \mathrm{keV}$, the APEC emission model is replaced with MeKa because APEC is not defined above $50 \mathrm{keV}$ in the current implementation of XSpec (Version 12.6.0k). For the thermal component, the temperature, abundance, redshift, and normalization are all varied. The individual abundances and redshifts in the $2 \mathrm{~T}$ model are tied together. The nonthermal photon index is initially fixed at $\Gamma=2$, typical of radio halos, and the normalization is allowed to vary; when the photon index is fit for, it is always fixed to the best-fit value before errors for other parameters are derived. In general, the photon index is poorly constrained, allowing for a wide range of normalizations, which are then less straightforward to evaluate. The purpose of fitting for the photon index is to make sure that we are not biased against detectable IC components with indices that differ from the fiducial value.

Because of complications arising at energies between 2 and $3 \mathrm{keV}$ (see Section 5.3.1), 
we perform these fits for both the $2-195 \mathrm{keV}$ (Table 5.3) and the $3-195 \mathrm{keV}$ spectral ranges (Table 5.4). The $E>2 \mathrm{keV}$ fits, at first glance, suggest that there may be evidence for a nonthermal component in a majority of HIFLUGCS clusters Many of the clusters with some evidence, at least at the $90 \%$ level, of a nonthermal excess are, unexpectedly, low temperature clusters without significant detections at BAT energies. In these cases, the nonthermal component is serving to "adjust" a problem at lower energies - due to either incompletely modeled low temperature components, an imperfectly calibrated response at the gold edge, or both. The significance of these instances will disappear from fits within a slightly higher energy range, while real nonthermal emission will become a higher proportion of the total flux and so this component should not greatly diminish in significance. A drastic reduction in the number of marginally detected nonthermal excesses is seen when comparing Tables 5.3 and 5.4; only 6 clusters are detected to have such emission at the $90 \%$ confidence level (statistical). These clusters will be discussed individually in Section 5.4.2.

While the 3-12 keV band avoids some possible systematic uncertainties with the $X M M$-Newton response and complications from cooler gas, the narrower range may reduce our ability to strongly constrain multi-temperature components in the spectra. One concern is that a weak nonthermal emission component might be indistinguishable from a purely thermal model with a slightly elevated temperature. Note, however, that the 3-12 keV band temperatures in Section 5.3.1 are typically only $\sim 0.3$ $\mathrm{keV}$ higher than the 2-12 keV temperatures. Therefore, the $1 \mathrm{~T}$ model temperatures should agree for the joint fits over both energy ranges, which is found to be the case in Figure 5.3. Temperatures derived from joint fits are consistent with those found using only the XMM-Newton spectra, for both energy ranges. For the most part, 
temperatures from the joint fit 3-195 keV fits are in good agreement with or slightly lower than the 3-12 keV temperatures. The contribution of the BAT data in this case is to somewhat lower the best-fit temperature, contrary to the expectation if a detectable nonthermal excess were present. The 3-195 keV nonthermal flux limits and possible detections (90\%, statistical) are shown in Figure 5.4.

\subsubsection{Individual Cases}

Six clusters have a formal detection of nonthermal emission in the $3-195 \mathrm{keV}$ band. Two of these 6 clusters are also in the top 3 of candidates for emission based on their BAT-only fits: A1651 and A2142. The other cluster in this top 3 - with the largest nonthermal normalization of all the clusters - is A2029, so we will include this cluster with the 6 "detected" clusters as worth some brief discussion. The clusters are listed in order of decreasing nonthermal flux.

A2029 (Fig. 5.5): This hot $(\sim 8 \mathrm{keV})$, cool core cluster has been studied in detail with Chandra (Clarke et al. 2004), who explore the interaction between cool gas and the radio AGN in the cluster center. The cluster is elongated but relatively regular; no evidence exists for current merger activity. Also, no evidence for an X-ray counterpart of the AGN is visible in the Chandra data. In addition to the radio jets, the core of the cluster is also host to an extended radio minihalo (Murgia et al. 2009). As with radio halos and relics, IC emission may be detectable from the minihalo if the magnetic field is small; Taylor et al. (1994) measured a lower limit of $B \gtrsim 0.11-0.19$ $\mu \mathrm{G}$ with Faraday RM observations of the jet. The implied magnetic field strength, if we take as the IC flux that found with the $2-195 \mathrm{keV}$ fit, is $B \sim 0.08 \mu \mathrm{G}$, roughly consistent with their field strength.

But have we really detected IC from the cluster core? The significance of the non- 


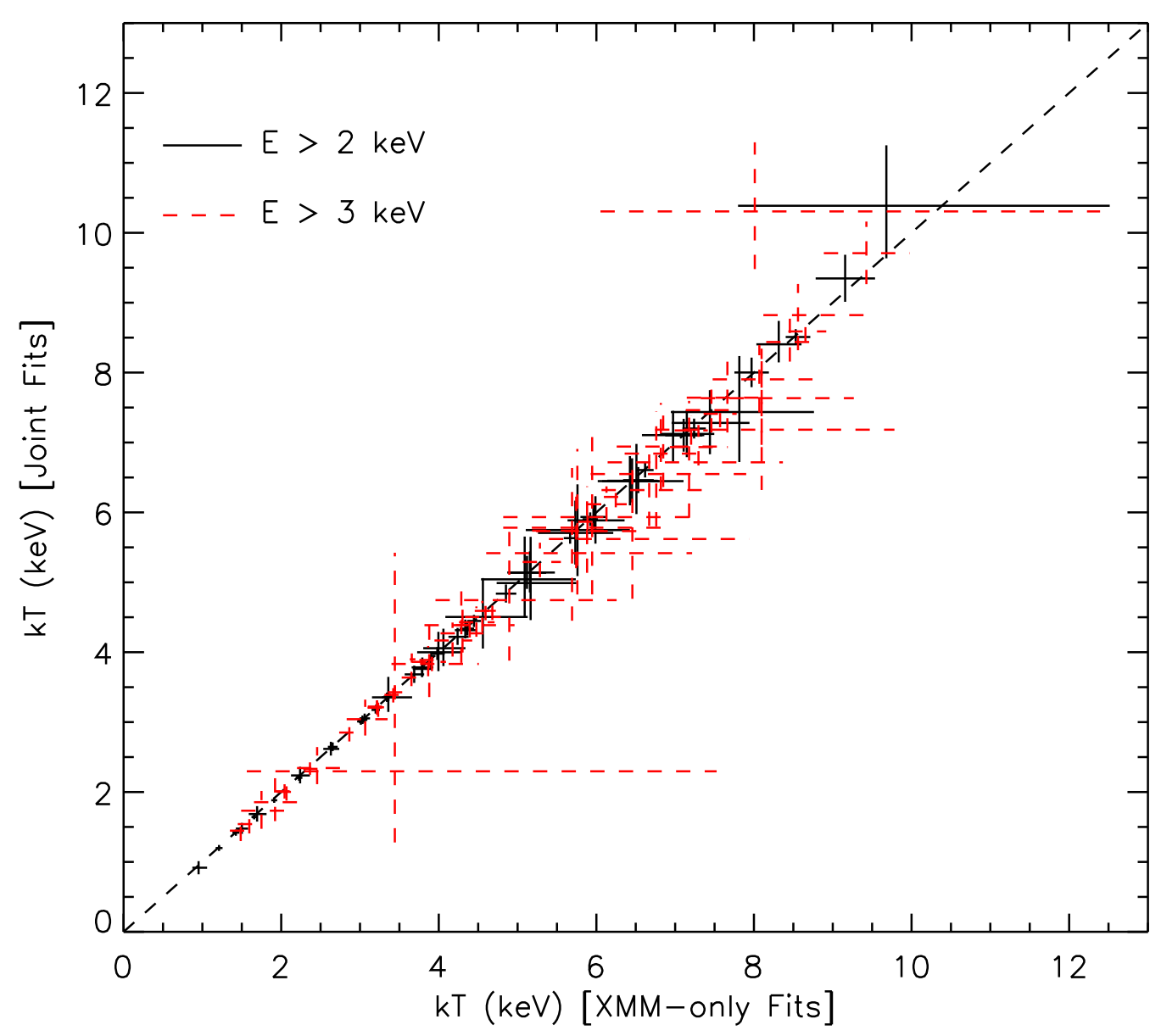

Fig. 5.3. - A comparison of best-fit temperature values in $1 \mathrm{~T}$ fits to only the $X M M$ Newton spectra (x-axis) and to the EPIC and BAT spectra simultaneously (y-axis). Solid lines indicate the $90 \%$ error interval for $E>2 \mathrm{keV}$ fits, dashed (red) lines for $E>$ $3 \mathrm{keV}$, and the dashed diagonal line represents equality between the two temperature determinations. Jointly fitting both datasets yields consistent temperatures to those derived only in the XMM-Newton band. Fitting over a slightly higher energy range $(E>3 \mathrm{keV})$, while increasing the average temperature by $\sim 0.3 \mathrm{keV}$ in the EPIC bandpass (see text), does not increase the joint fit temperatures as much; note how the dashed points fall slightly below equality for moderately hot clusters. 


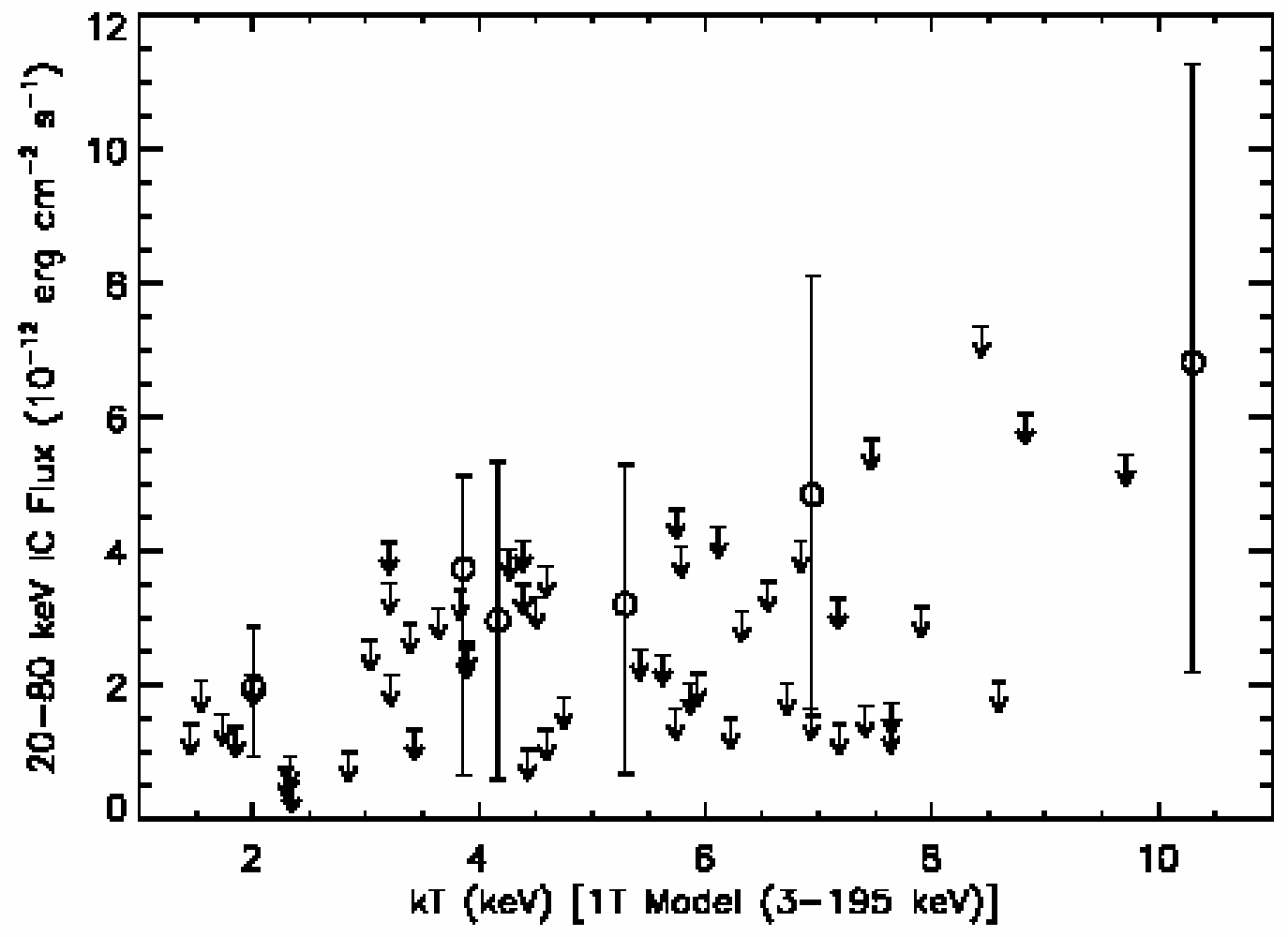

Fig. 5.4. - Upper limits and measurements of the nonthermal spectral component in the 3-195 keV joint fits as a function of cluster temperature. Limits and error bars indicate the $90 \%$ confidence interval without considering the impact of systematic uncertainties. In general, an excess attributable to IC emission is not observed, and the few detections, discussed individually in the text, have marginal statistical significance. 


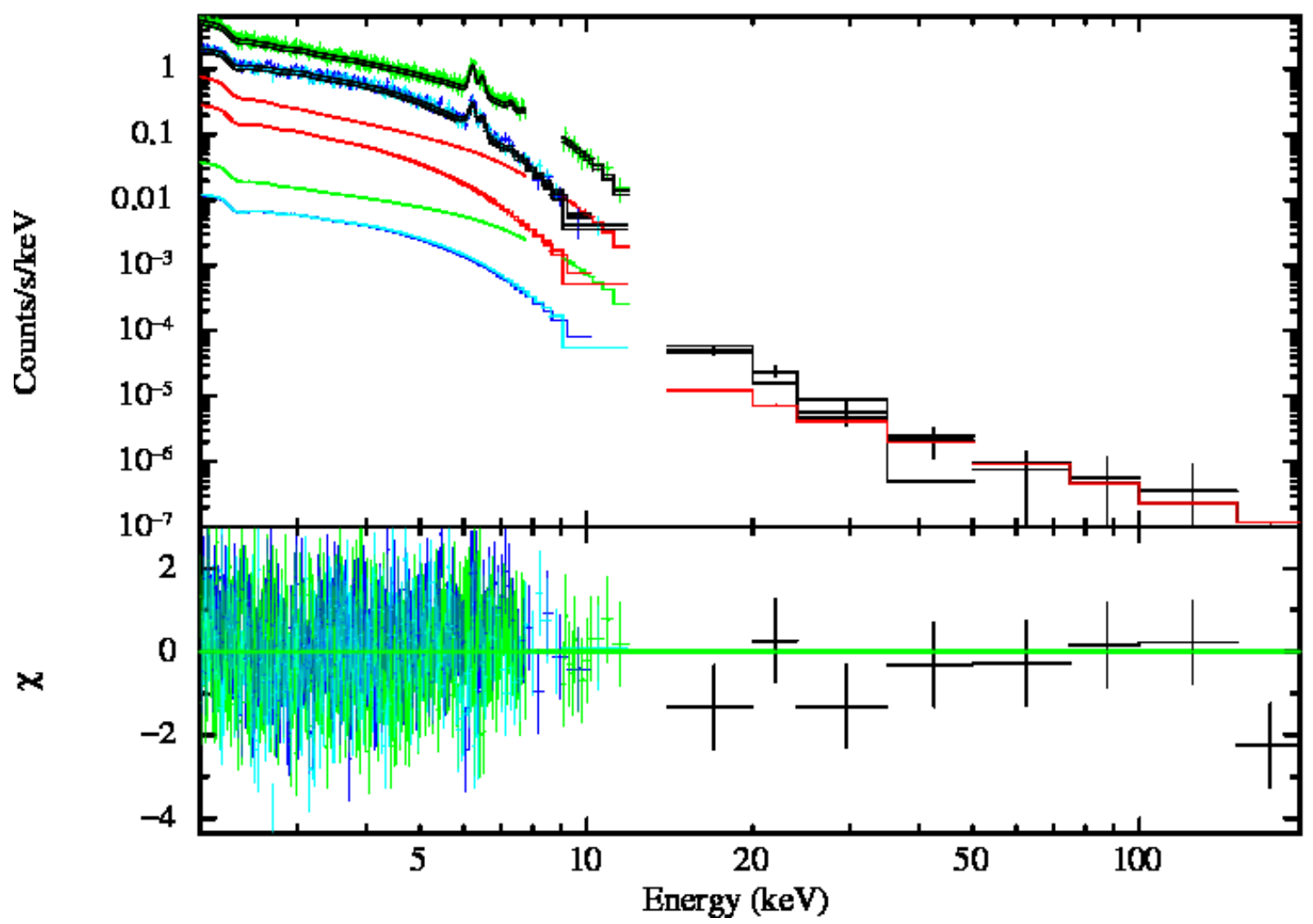

Fig. 5.5.- Abell 2029: The T+IC model simultaneous fit to the EPIC $(E<12 \mathrm{keV})$ and BAT $(E>14 \mathrm{keV})$ spectra. The EPIC-pn spectrum and residuals are in green, and the MOS 1 and 2 spectra/residuals are in dark and light blue, respectively. The like-colored lines below these spectra show the CXB model contribution. The total model fit and thermal contribution is represented by the black histogram, and the red lines represent the nonthermal $(\Gamma=2)$ spectral component. 
thermal component completely disappears in the 3-195 keV fit. Also, the $2 \mathrm{~T}$ formally provides a better fit to the $2-195 \mathrm{keV}$ spectrum where the nonthermal component is detected. The second temperature component, $\sim 0.3 \mathrm{keV}$, is consistent with a low temperature component of $0.11 \mathrm{keV}$ observed by Clarke et al. (2004). Given these results, it is more likely that the nonthermal component is trying to mimic the low $k T$ cool core component in the $2-3 \mathrm{keV}$ range, since its significance disappears if this energy range is ignored. However, it is worth noting that the BAT data do generally support hard emission at higher energies, although at low signal-to-noise. Such hard emission could be due, on the other hand, to heavily obscured AGN X-ray emission from the central source at a level not quite detectable in the 58-month survey. The spatial distribution of BAT emission is consistent with that from a point source in all bands.

A1651 (Fig. 5.6): This cluster has a weak cool core, which means that while there is no significant temperature gradient in the center, the cooling time of the gas in the center is short (Hudson et al. 2010). Given the similarity between its BAT data and that of A2029, an obscured AGN of similar flux could be responsible for the marginally detected positive flux in the higher energy bands. However, in this case the $\mathrm{T}+\mathrm{IC}$ model is a significantly better fit than is the $2 \mathrm{~T}$ model; $\Delta \chi^{2}$ improves by $9(2-195 \mathrm{keV})$ and $6(3-195 \mathrm{keV})$ over the $1 \mathrm{~T}$ and $2 \mathrm{~T}$ models. If there were no hard excess, the probability that the 6 highest energy bands measure flux above the thermal component is $\left(\frac{1}{2}\right)^{6}$, or $1.6 \%$, which is not impressive in a sample of 58 clusters. The BAT spectrum is certainly suggestive, but considering the excess is not significant at the $3 \sigma$ level for the $3-195 \mathrm{keV}$ fit, and only just at this level in the $2-195 \mathrm{keV}$ fit - without including systematic uncertainties - we cannot claim to have detected a nonthermal component in this cluster. However, the evidence is perhaps strongest in 
this case, which is contrary to the expectation that such an excess is most likely in a merging cluster, particularly one with a radio halo or relic.

A2142 (Fig. 5.7): As the hottest cluster in the sample, the BAT is easily able to detect its high energy emission, which we might expect to exhibit a nonthermal excess since it also hosts a radio halo (Giovannini \& Feretti 2000). Both the $\mathrm{T}+\mathrm{IC}$ and $2 \mathrm{~T}$ models indicate that hard excess emission may be present; in the latter case, the second temperature component is unphysically high, acquiring the highest allowed temperature value. However, Nevalainen et al. (2004) estimate that 2 Seyfert galaxy nuclei within $17^{\prime}$ of the cluster center contribute $\sim 30 \%$ of the hard band emission detected by Beppo-SAX; a similar amount of contamination would be expected in the BAT spectrum. Unfortunately, the XMM-Newton observation places this cluster right on the edge of the FOV, so over half $(55.6 \%$, based on a comparison with a pointed ROSAT PSPC image) of the soft band emission is missing from the EPIC spectra. We rescale the XMM-Newton spectra to correct for the lost flux; the BAT source is equivalent to a point source, so it is not possible to correct the BAT emission for the XMM-Newton FOV. The correction to the XMM-Newton flux could be off by a sizable factor if the $E>2 \mathrm{keV}$ emission is distributed differently than the $E<2 \mathrm{keV}$ emission where ROSAT is sensitive. The significance of the nonthermal excess here is only at the $2 \sigma$ level, mainly due to the poor statistics at XMM-Newton energies. While inconclusive, the BAT spectrum warrants further analysis When better data is available below $12 \mathrm{keV}$.

A3112 (Fig. 5.8): Using both Chandra and XMM-Newton data, Bonamente et al. (2009) have claimed to see both a hard and soft excess, which could have a nonthermal origin. If this is the correct interpretation of these spectra, the IC excess would be clearly detectable in the BAT spectrum given our sensitivity. While a nonther- 


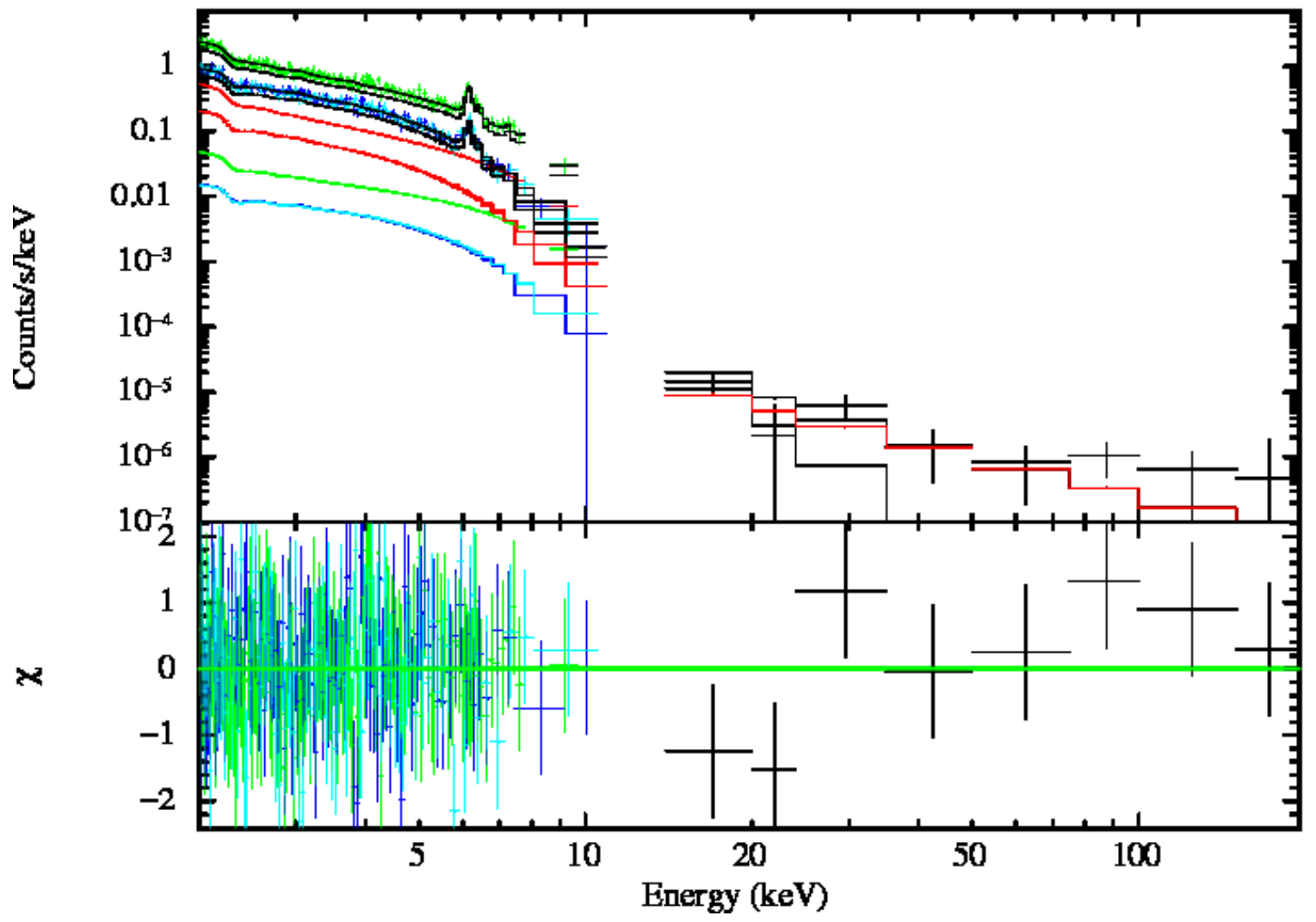

Fig. 5.6.- Abell 1651: The T+IC model simultaneous fit to the EPIC $(E<12 \mathrm{keV})$ and $\operatorname{BAT}(E>14 \mathrm{keV})$ spectra. The notation is identical to Figure 5.5. 


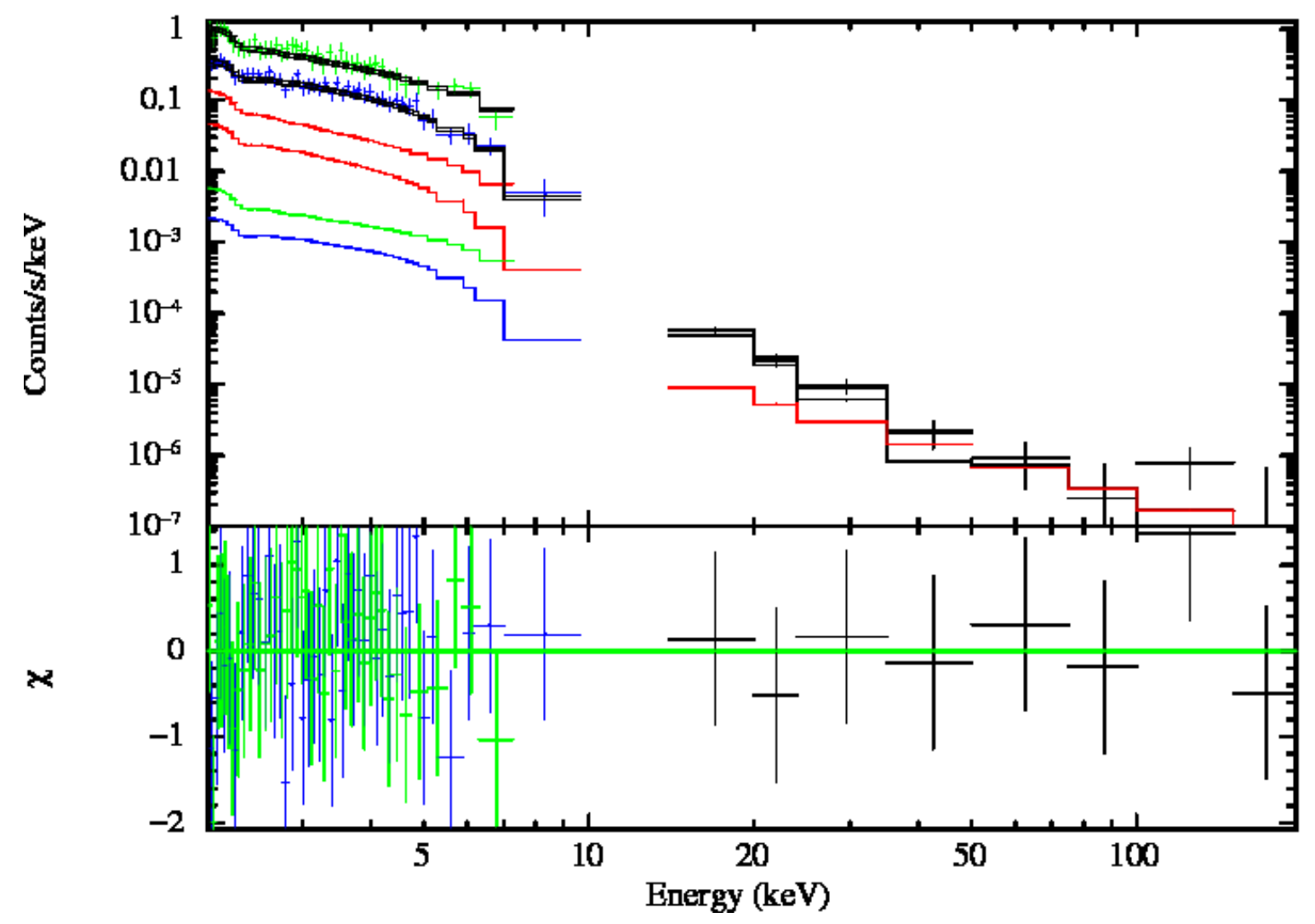

Fig. 5.7.- Abell 2142: The T+IC model simultaneous fit to the EPIC $(E<12 \mathrm{keV})$ and BAT $(E>14 \mathrm{keV})$ spectra. The notation is identical to Figure 5.5. 
mal component is detected in our joint fits, it has well below the predicted flux of Bonamente et al. (2009); our $3 \sigma$ upper limit on the nonthermal normalization, using a photon index Gamma $=1.8$ that matches their best-fit value, is 3 times lower than their claim. The quality of our $1 \mathrm{~T}$ model fits is significantly less than for either the $2 \mathrm{~T}$ or $\mathrm{T}+\mathrm{IC}$ models, though those fits are of similar quality. Fitting over $2-195 \mathrm{keV}$, the $2 \mathrm{~T}$ model provides a formally better fit, while from $3-195 \mathrm{keV}$ the $\mathrm{T}+\mathrm{IC}$ is better. A nonthermal excess may in fact exist in this cluster, but an equally likely scenario is that the ICM here is less isothermal than is typical of clusters, requiring several temperature components to adequately explain the cluster emission. In any case, the BAT data do not argue strongly in favor of an IC interpretation for the excess emission above $\sim 7 \mathrm{keV}$. A more detailed exploration of the spatial and thermal structure at $E<12 \mathrm{keV}$ is certainly warranted.

A1367 (Fig. 5.9): This cluster hosts a radio relic in its outskirts (Gavazzi \& Trinchieri 1983), and so IC emission is expected at some level in the radio relic region; however, the XMM-Newton/Swift extraction region does not contain the relic, so we are unable to address the magnetic field strength. Using RXTE, Henriksen \& Mushotzky (2001) potentially detect a nonthermal component, although a two temperature fit better describes their spectrum. The marginally detected IC emission we see is consistent with their nonthermal flux, whether we use a photon index of 2.0 or their value (based on the spectrum of the radio relic) of 2.9. Our $2 \mathrm{~T}$ model fits are generally as good as the $\mathrm{T}+\mathrm{IC}$ model fits, and given the marginally detected fluxes in the BAT bands, a $2 \mathrm{~T}$ description of the ICM in this early stage, forming cluster cannot be ruled out.

A2589 83 Fornax: Neither of the BAT spectra of these clusters show particular evidence that that they have detected emission of any kind in any band. The first 2 


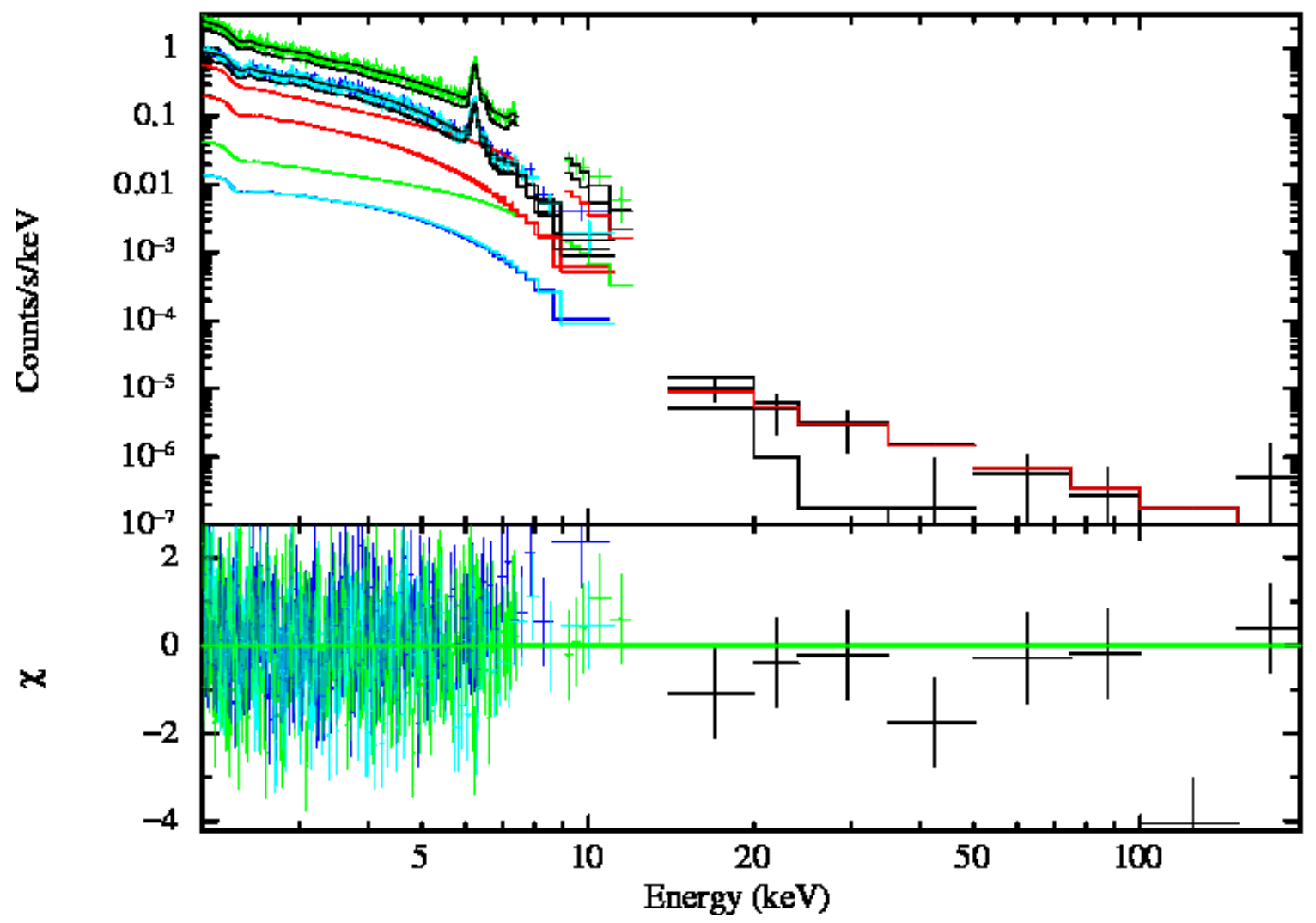

Fig. 5.8. - Abell 3112: The T+IC model simultaneous fit to the EPIC $(E<12 \mathrm{keV})$ and $\operatorname{BAT}(E>14 \mathrm{keV})$ spectra. The notation is identical to Figure 5.5. 


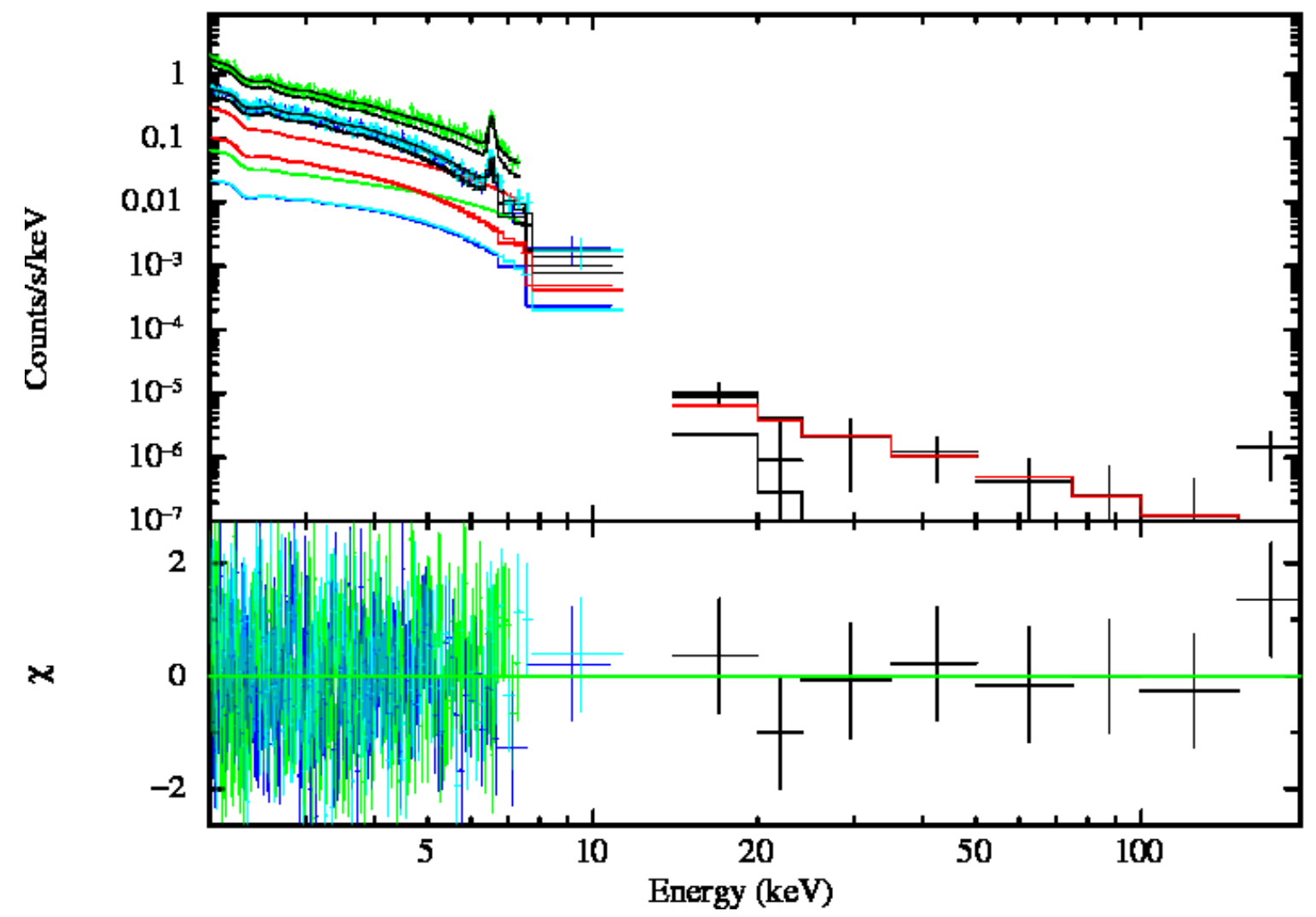

Fig. 5.9. - Abell 1367: The T+IC model simultaneous fit to the EPIC $(E<12 \mathrm{keV})$ and BAT $(E>14 \mathrm{keV})$ spectra. The notation is identical to Figure 5.5. 
bands of A2589's spectrum are just inconsistent with zero flux at the $1 \sigma$ level, but a marginal detection in these bands is consistent with the thermal component. In both cases, the BAT spectrum is not sensitive enough to exclude the nonthermal component driven by the XMM-Newton data; since the BAT data do not further constrain the nonthermal component in these cases, we will not discuss these clusters further.

\subsubsection{Upper Limits}

While some evidence for nonthermal emission is present in several of the HIFLUGCS clusters, in none of these cases is a significant excess indicated by both the BAT and EPIC spectra that could not plausibly be explained by a multi-temperature state of the ICM. In many cases, the BAT spectra simply lacked the signal-to-noise to meaningfully constrain the existence of excess emission; we therefore derive upper limits for a nonthermal component in our joint spectra. Three limits are presented for each energy range (2-195 keV and 3-195 keV) considered: a 90\% confidence level limit including systematic uncertainties in $f_{\mathrm{CN}}$ and the EPIC backgrounds, as described in Section 5.2.1, and two $3 \sigma$ limits, without systematic uncertainties included, for our fiducial photon index of $\Gamma=2$ and for the best-fit value of $\Gamma$. After fitting for $\Gamma$, it is then fixed at that value when the upper limit is computed. The systematic terms are included in the $90 \%$ limits as described in Wik et al. (2009). Upper limits are reported as 20-80 keV fluxes in units of $10^{-12} \mathrm{erg}^{-2} \mathrm{~s}^{-1}$ in Table 5.5. In some instances, usually for lower temperature clusters, the $90 \%$ limit exceeds the $3 \sigma$ limits; in these fits, the systematic uncertainties in $f_{\mathrm{CN}}$ and/or the EPIC background dominates over the statistical uncertainty in the spectra. For example, in a low temperature cluster lowering the EPIC backgrounds significantly hardens the spectra, while modifying 
$f_{\mathrm{CN}}$ such that already poorly constraining BAT fluxes are $10 \%$ higher, will allow a much larger IC-like component to fit the data than would be allowed statistically. In hotter clusters, adjusting the background has less of an effect on their spectral shape, and because they are hot they tend to be more significantly detected by the BAT, so that modifying $f_{\mathrm{CN}}$ cannot drastically affect the nonthermal component.

\subsection{Joint Fits to Stacked EPIC-BAT Spectra}

In some clusters, as noted above, hints of a nonthermal excess are present, even if we cannot argue for their definite detection. If the excess does exist in several clusters, but just below the detection threshold, we may be able to increase the signal-to-noise enough for a statistical detection by stacking the cluster spectra. For simplicity, we stack only the EPIC-pn XMM-Newton spectra, which have the highest sensitivity especially at higher energies. Stacking the MOS spectra would be complicated by the variable pn/MOS cross-calibration factor and the fact that 3 of the cluster MOS spectra have been excluded from our analysis. Both the pn and BAT spectra are straightforwardly summed, as are the pn backgrounds, and their errors are propagated. Because the same response matrix is used for all the BAT spectra, we are able to use this unmodified file with stacked spectrum. To create an average response matrix for use with the stacked pn spectrum, we first multiply the individual redistribution matrices by their respective auxiliary response files, which contain the effective area per incoming photon energy. Then, a weighted average is performed on the new response files, with weighting factors proportional to each spectrum's 2-7 keV count rate. This procedure ensures that the final response matrix will best represent the instrumental response for the majority of photons. In any case, an unweighted response file was also created and no significantly different results were produced when 
using it. The CXB model normalizations were summed and included in the spectral fits.

In all, we create 8 stacked spectra based on different groupings of the $58 \mathrm{HI}-$ FLUGCS clusters for which we have XMM-Newton data: "All" clusters, "Hot" ( $k T>7 \mathrm{keV}$, from the $2-12 \mathrm{keV}$ fits), "cool" $(k T<7 \mathrm{keV})$, "Radio" clusters hosting a radio halo or relic, "No Radio" clusters that do not host a known halo or relic, non-cool-core clusters ("NCC"), strong cool core clusters ("SCC"), and weak cool core clusters ("WCC"), as defined by Hudson et al. (2010) and listed in Table 5.1. These categories are designed to separate the sample into subgroups which might have different average levels of nonthermal emission. For example, IC emission must exist at some level in clusters with a radio halo or relic, but may not be present in clusters more generally. Thus, we might expect the "Radio" clusters to preferentially have nonthermal excesses, which are enhanced when they are stacked together and not diluted by the additional spectra from "No Radio" clusters that have no such excess.

Because these clusters span a large range of temperatures and redshifts, it is not appropriate to model the summed spectra with a single or even several temperature model for the thermal component. Instead, we build multi-temperature models from the previous spectral fits, for which we keep the spectral shape fixed and only allow the overall normalization to vary during fits to the stacked spectra. We consider the XMM-Newton-only single temperature fits (Table 5.2) derived from 2-12 keV $\left(1 \mathrm{~T}_{\mathrm{X},>2}\right)$ and from $3-12 \mathrm{keV}\left(1 \mathrm{~T}_{\mathrm{X},>3}\right)$, and the single $\left(1 \mathrm{~T}_{\mathrm{J}}\right)$ and double $\left(2 \mathrm{~T}_{\mathrm{J}}\right)$ temperature fits derived from 2-195 keV (Table 5.3). To search for nonthermal emission in the stacked spectra, a power law model is added to represent the IC component and the normalization of the thermal model is allowed to vary. Ideally, the shape of 
the thermal component would be able to adjust to accommodate the IC signal, as it effectively does in the individual joint fits via the temperature parameter. However, the nonthermal flux below $12 \mathrm{keV}$ will be small and should not cause the temperature to change in any significant way. For the $2 \mathrm{~T}_{\mathrm{J}}$ model, we want to avoid including unphysical temperature components that may have been driven by calibration features at the edges of the spectral range in the individual $2 \mathrm{~T}$ fits. A low temperature $(\lesssim 2$ $\mathrm{keV}$ ) component's emission measure may cause $<2 \mathrm{keV}$ emission to be significantly overestimated in order to better fit the gold edge, for example. Similarly, a slight under-subtraction of the XMM-Newton background or positive fluxes in the higher energy BAT bands may lead to unrealistically high temperatures. In Figure 5.10, we plot the temperature values for this model relative to the $1 \mathrm{~T}_{\mathrm{J}}$ model temperatures. We have removed unphysical temperature components from both the $2 \mathrm{~T}_{\mathrm{J}}$ model and the figure; the best-fit single temperature model is used in place of the $2 \mathrm{~T}$ model for those clusters, which are represented by blue circles in Figure 5.10. Unphysical temperature components were found to have $k T>14 \mathrm{keV}$ (except for A0754, for which this component is plausible given the hot, disturbed state of its ICM) and $k T<2.1$ $\mathrm{keV}$, if their $1 \mathrm{~T}_{\mathrm{J}}$ temperature is greater than $3.5 \mathrm{keV}$. In general, this latter cut eliminates temperature components that significantly over-predict the $0.5 \mathrm{keV}<E<2$ $\mathrm{keV}$ emission.

Thermal and thermal plus nonthermal fits to the stacked spectra are given in Table 5.6. The normalization of the thermal component in the "T Model-only" fits is not shown, only its $\chi^{2}$ value for comparison purposes. For the "T $\mathrm{T}_{\text {Model }}+\mathrm{IC}$ " fits, the photon index is fixed to $\Gamma=2$ as was done previously for the joint fits. The last 3 columns report the " $\mathrm{T}_{\text {Model }}+\mathrm{IC}$ " fits with $\Gamma$ as a free parameter; however, its value is fixed when errors are computed. The photon index was initialized as 


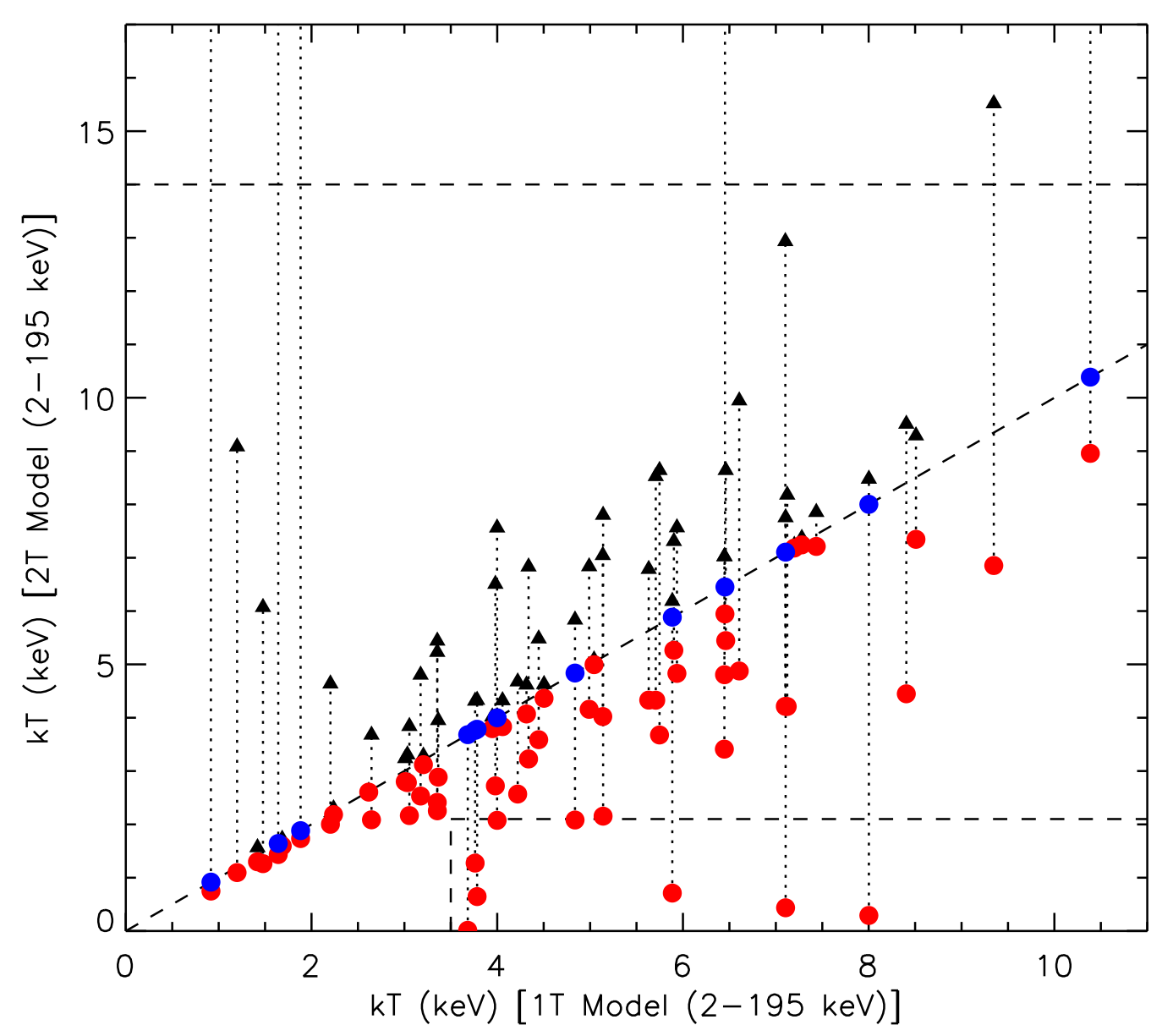

Fig. 5.10.- The two temperature values in the $2 \mathrm{~T}$ fits $(2-195 \mathrm{keV})$ with respect to the single temperature fit for each cluster over the same range. High (triangles, black) and low (circles, red) temperature values in the $2 \mathrm{~T}$ model for the same cluster are connected by dotted lines for clarity. Blue circles represent fits with an unphysical best-fit $2 \mathrm{~T}$ model, with either unrealistically high temperatures $(k T>14 \mathrm{keV}$, except for A0754) or a low temperature component that over-predicts the emission below $2 \mathrm{keV}$. (These have $k T \lesssim 2.1 \mathrm{keV}$ for clusters with single temperatures of $3.5 \mathrm{keV}$ or hotter.) These excluded regions are indicated by dashed lines in the figure, and the diagonal dashed line represents equality of $1 \mathrm{~T}$ and $2 \mathrm{~T}$ temperatures. These temperature values are used to build the $2 \mathrm{~T}_{\mathrm{J}}$ model used in fits to the stacked spectra. 
$\Gamma=1$, so for spectra with no particularly strong indication of nonthermal emission, the best-fit normalization was set to zero and the photon index kept at or near its initialized value; this explains why so many of the "best-fit" photon indices presented in the table are near unity. In the case of very large values of $\Gamma$, the nonthermal component is attempting to either represent incompletely modeled soft emission from low temperature gas or correct an imperfectly calibrated gold edge. Even though these normalizations are large and quite significant, they are so steep that the flux at hard energies is negligible and does not represent an IC excess. If $<2 \mathrm{keV}$ emission where included in the fits, these large $\Gamma$ values would disappear as they would vastly over-predict the soft emission.

In Figure 5.11, the jointly fit stacked spectra for all 58 clusters is shown with the $1 \mathrm{~T}_{\mathrm{X},>2}$ model. The best-fit model normalization agrees with its expected value to better than $1 \%$, as do all the model fits without an IC component, indicating that the average pn response is accurate. Also, a difference in the spectra shape appears below $3 \mathrm{keV}$, visible in the residuals, that highlights the problem with including this emission in the fits. The BAT data are well represented by this model, even though the temperature models were derived from fits to the XMM-Newton spectra alone. The regular pattern in the BAT residuals is likely real, and is apparent in most of the spectra of hot clusters such as Coma (see Chapter 4). When considering only one cluster, it seemed reasonable that this residual pattern could simply be due to chance. The pattern reappears in many of the individual joint fits however, indicative of a systematic problem. Because the BAT flux calibration is dominated by normalizing to the Crab flux in each band, these fluxes are really only accurate for objects with a spectral slope similar to the Crab's. At these energies, cluster spectra are quite steep even for the hottest temperatures, so some miss-calibration would be expected. Most 


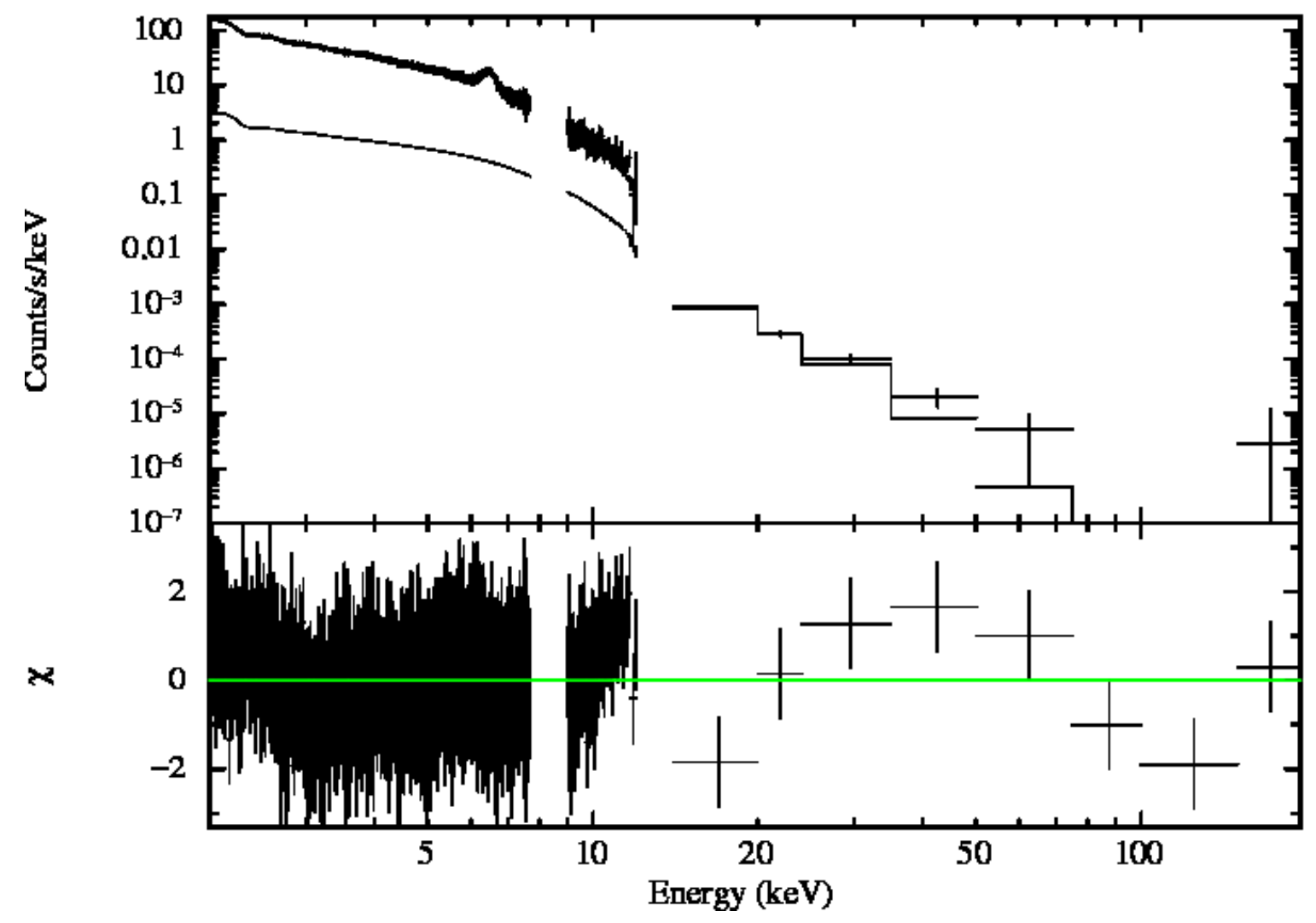

Fig. 5.11.- The stacked spectrum of all 58 clusters with the combined single temperature model fit $\left(1 \mathrm{~T}_{\mathrm{X},>2}\right)$. The EPIC-pn spectrum $(E<12 \mathrm{keV})$ and BAT spectrum $(E>14 \mathrm{keV})$ are shown in the top panel, and their residuals in the lower panel. The CXB contribution appears below the EPIC-pn data. The problems between 2$3 \mathrm{keV}$ (described in the text) clearly show up in the residuals, as does a potential problem with low energy BAT fluxes. The combined single temperature model determined from the $2-12 \mathrm{keV}$ fits is sufficient to explain the summed BAT spectrum; no nonthermal excess is obvious. 
likely, the first and possibly second energy bands have underestimated fluxes, owing to the rapid rise of the instrumental response with energy; clusters have proportionately more emission at the lower energy part of the band than does the Crab, and so the internal band response is miss-calibrated - weighting the higher energy part of the response more strongly than is appropriate for thermal emission. While this certainly affects our results, the only solution is develop a detailed response matrix model for the survey data. Unfortunately, the detailed spectral response for the Swift data currently has much larger uncertainties than the Crab spectrum.

In general, the addition of a nonthermal component to these spectra does not significantly improve the fits in Table 5.6, except for the "Radio" subsample. The best-fit nonthermal plus $\mathrm{T}_{\mathrm{X},>2}$ model is shown in Figure 5.12. For comparison, the $\mathrm{T}_{\mathrm{X},>2}$ and $2 \mathrm{~T}_{\mathrm{J}}$ fits with no IC component are shown in Figure 5.13 and Figure 5.14, respectively. The nonthermal component, plotted as a red line in the figure, becomes competitive with the thermal emission in the $35-50 \mathrm{keV}$ band, where a somewhat significant excess is present in thermal-only model fits. By contrast, the "No Radio" subsample shows no evidence for an excess at hard energies (Fig. 5.15).

Ignoring systematic uncertainties, the nonthermal signature is detected with $3.5 \sigma$ confidence using the $\mathrm{T}_{\mathrm{X},>2}$ model and $2.3 \sigma$ with the $\mathrm{T}_{\mathrm{X},>3}$ model. Including an $f_{\mathrm{CN}}$ uncertainty of $3.33 \%$ - the nominal $10 \%$ uncertainty is unlikely to occur in the same direction in all clusters, so it approximately reduced by the square root of the number of stacked clusters - reduces the significances to $2.9 \sigma$ and $1.8 \sigma$, respectively. While only a marginal detection, considering the $\mathrm{T}_{\mathrm{X},>3}$ model fit, which should be less biased, it is encouraging that the subsample that would be expected to contain the largest nonthermal excess shows the most significant evidence for such an excess. 


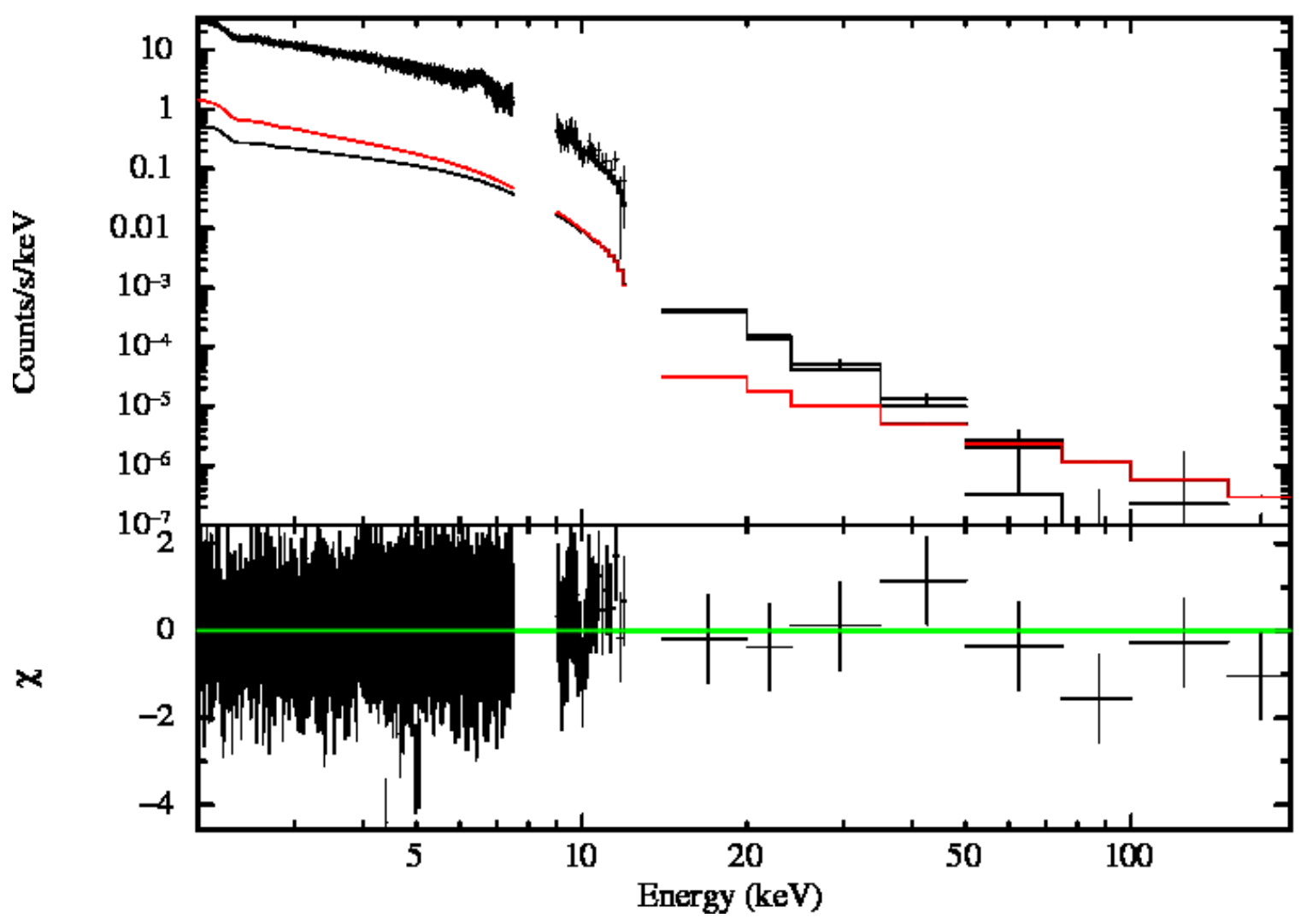

Fig. 5.12.- The stacked spectrum of all clusters with large-scale, diffuse radio halos or relics. General features of the plot are the same as Figure 5.11. The red line represents the best-fit nonthermal model with photon index $\Gamma=2$, and the lower black line shows the $1 \mathrm{~T}_{\mathrm{X},>2}$ thermal model. 


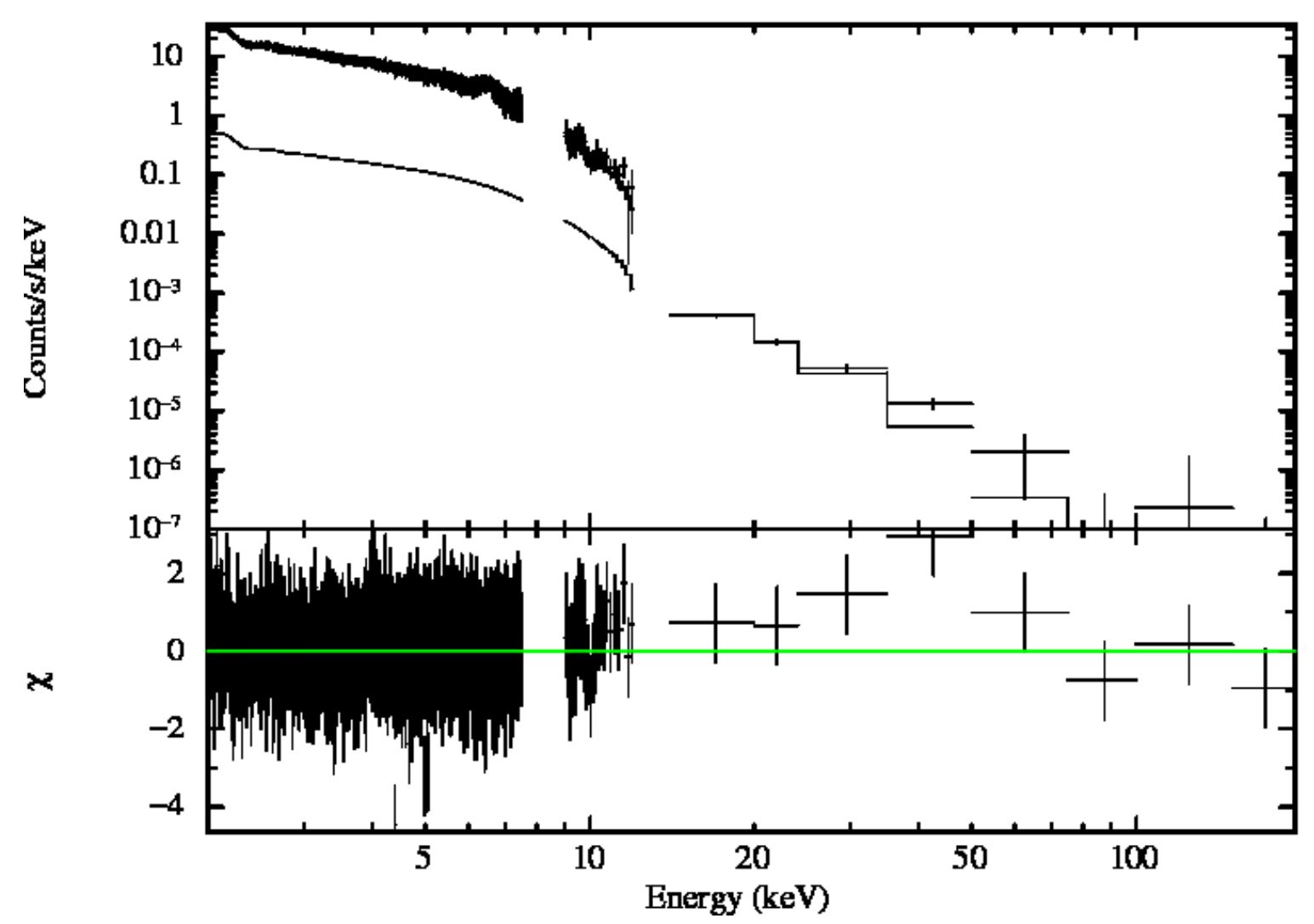

Fig. 5.13. - The stacked spectrum of all clusters with large-scale, diffuse radio halos or relics with the combined single temperature model fit $\left(1 \mathrm{~T}_{\mathrm{X},>2}\right)$. General features of the plot are the same as Figure 5.11. A slight excess is apparent in the BAT spectrum, due to either a nonthermal spectral component (see Fig. 5.12) or a significant multitemperature structure in the individual clusters (see Fig. 5.14) 


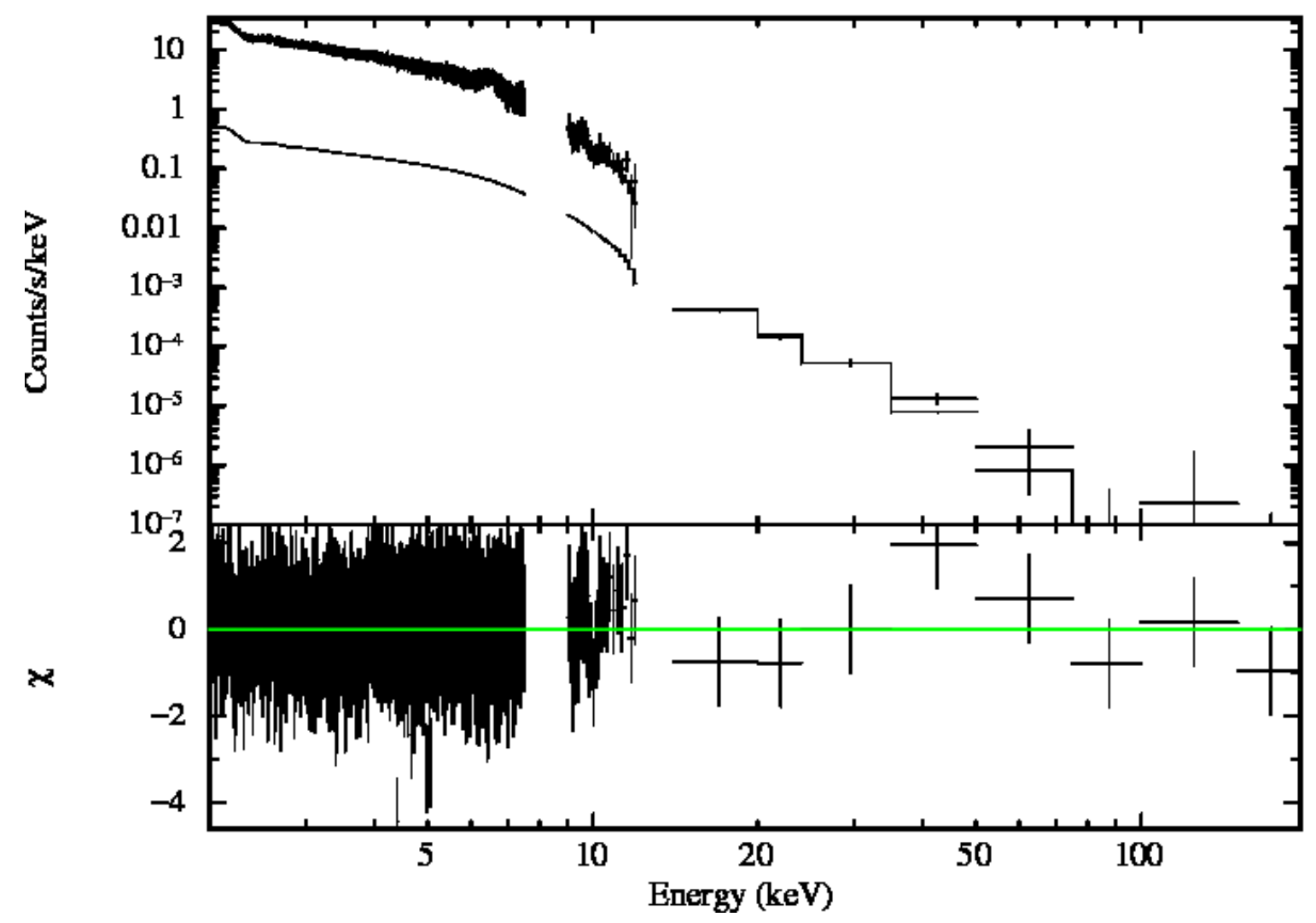

Fig. 5.14.- The stacked spectrum of all clusters with large-scale, diffuse radio halos or relics with the combined double temperature model fit $\left(2 \mathrm{~T}_{\mathrm{J}}\right)$. General features of the plot are the same as Figure 5.11. The combined $2 \mathrm{~T}_{\mathrm{J}}$ can explain the slight excess seen when the single temperature model $\left(1 \mathrm{~T}_{\mathrm{X},>2}\right)$ is considered. 


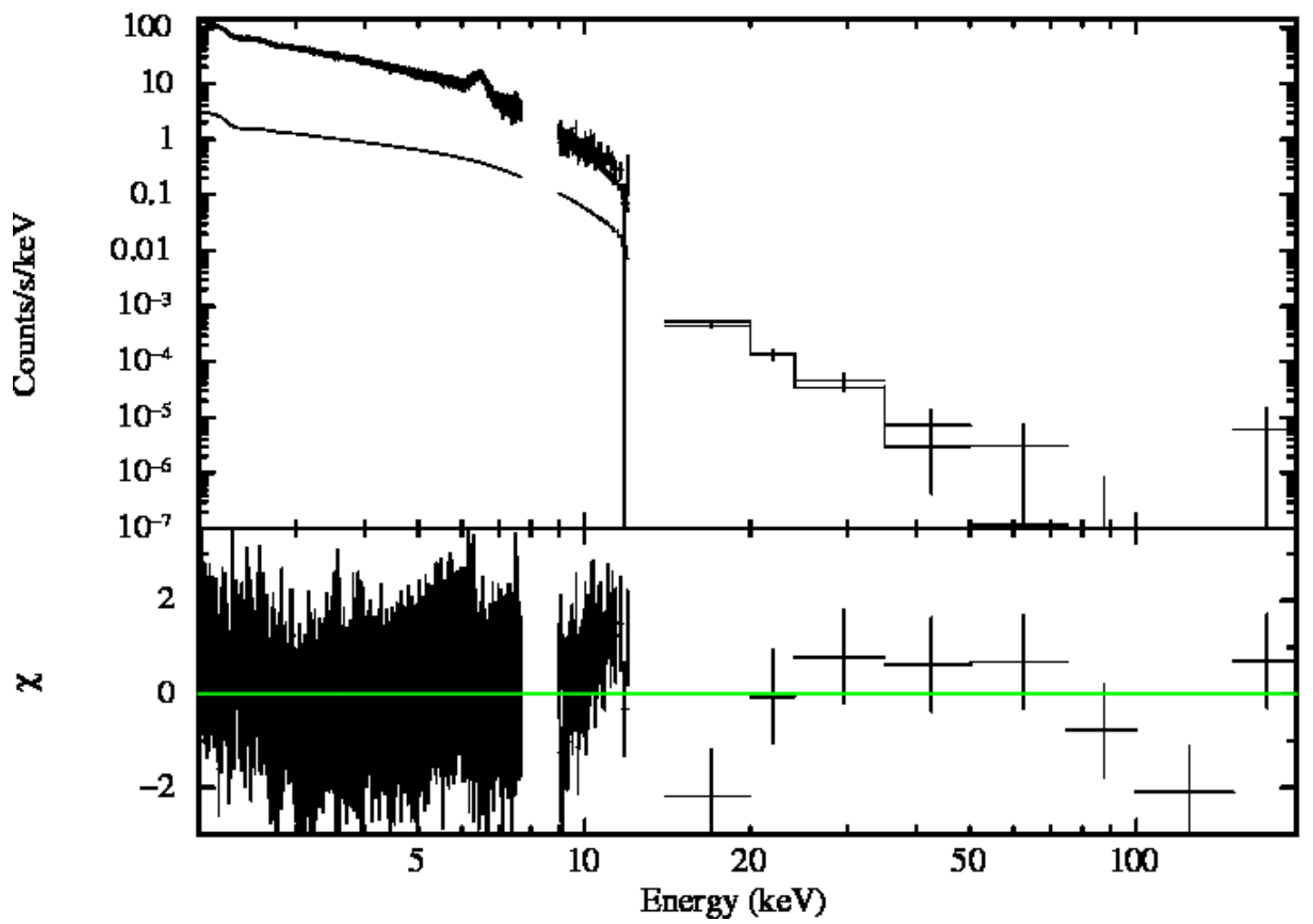

Fig. 5.15. - The stacked spectrum of all clusters without diffuse radio emission shown with the combined single temperature model fit $\left(1 \mathrm{~T}_{\mathrm{X},>2}\right)$. General features of the plot are the same as Figure 5.11. 


\subsection{Implications and Discussion}

In this work, we characterized the hard X-ray emission from HIFLUGCS, a sample of the brightest galaxy clusters outside the Galactic plane. For the 58 out of 63 clusters with usable XMM-Newton data, we searched for excesses over the thermal emission from gas in the ICM in data from the 58-month Swift BAT all-sky survey. EPIC and BAT spectra were extracted from identical regions and carefully calibrated to allow straightforward joint fits that simultaneously constrain the thermal and nonthermal emission in both spectra. We first considered fitting over an energy range of $2-195 \mathrm{keV}$ but found that low temperature gas and the gold edge in the XMM-Newton spectra could lead to false detections. Ignoring the $2-3 \mathrm{keV}$ data resolved this issue, although a somewhat weaker constraint on the thermal component reduced our overall sensitivity.

From the 3-195 keV fits, six clusters were found to have marginal evidence for a nonthermal excess, although none of these were deemed significant enough to claim a detection, especially considering systematic uncertainties in the EPIC background and EPIC-BAT cross calibration normalizations. We then stacked the spectra to look for a significant statistical detection of nonthermal emission in the HIFLUGCS sample. Unfortunately, the stacked spectra revealed no definitive excess. Stacking subsamples of the HIFLUGCS clusters returned similar results, except for a tantalizing but still marginal detection of a nonthermal component in the stacked spectrum of all clusters that host radio halos and/or relics - the very clusters that are most expected to have detectable IC emission.

While some excesses in the stacked spectra are tantalizing, equally good, and sometimes better, fits result when the $2 \mathrm{~T}_{\mathrm{J}}$ model is used. Since only the normalization is allowed to vary in these fits, it is hard to justify why the addition of an IC component really provides a better description of the data, especially if the improvement in $\chi^{2}$ is 
minor. Note that this comparison is only fair because the 2T models are all physically reasonable descriptions of the ICM, otherwise we may be inappropriately modeling nonthermal emission with an incorrect thermal component. The upper limits on nonthermal emission in the stacked spectra, when applied on average to the clusters making up the stacked sample, are more constraining than limits from individual fits. The typical $90 \%$ confidence level upper limit on the cumulative IC flux in the stacked spectra is $\sim 2 \times 10^{-12} \mathrm{erg} \mathrm{cm}^{-2} \mathrm{~s}^{-1}$ in the $20-80 \mathrm{keV}$ band, which translates to an average flux per cluster 9 to 58 times lower than this limit.

These results seem to be in conflict with an analysis of a similar sample of clusters observed by Beppo-SAX (Nevalainen et al. 2004), which found systematic if marginal excesses. Actually, these previous IC flux estimates are more or less consistent with our results in the $2-195 \mathrm{keV}$ range, as are the temperatures of the thermal component. However, over the 3-195 keV energy range, the 90\% error interval for nearly all the excesses include zero. This result is at least partly due to slightly higher best-fit temperatures (see Section 5.3.1 for a more detailed discussion). Since clusters are not isothermal, harder spectra such as those from the BAT will contain proportionately more photons from higher temperature gas. An example of this bias can be seen in the stacked spectrum of Nevalainen et al. (2004); they observe a highly significant nonthermal excess, but the steep IC component necessary to explain it would lead to detectable amounts of nonthermal emission at softer energies, which is not seen. The authors interpret this as evidence that the nonthermal emission is significantly extended. Our BAT data test this possibility, as extended emission is both detectable and not detected by the BAT beyond that produced by the thermal gas. Thus, it is unlikely that the nonthermal emission is very highly extended and strong. In fact, the steep excess in their spectrum is exactly what would be expected for a strongly multi- 
temperature thermal structure - which naturally results when many clusters spanning a broad range in temperature are summed - that is modeled as a single temperature component. When we model our stacked spectrum this way, we find a temperature consistent with the average temperature of our clusters and a very significant, steep $(\Gamma \sim 2.8)$ power law component, identical to their best-fit photon index. But the thermal component, determined at hard energies, will be more highly weighted by hotter clusters, whose emission dominates. If a single temperature component is used to model the thermal emission for such a summed spectrum, then at the very least the temperature needs to be fixed to the weighted-average value in the band in which the hard excess emission is expected to be found. For example, in our sample, the count rate weighted-average temperature jumps from $5.6 \mathrm{keV}$, when weighted by the 2-7 keV count rate, to $7.1 \mathrm{keV}$ when the $14-50 \mathrm{keV}$ count rate is used. Here we have employed the temperatures determined from the $2-12 \mathrm{keV}$ fits. Regardless, the proper procedure is to use a truly multi-temperature model based on the temperatures of the constituent clusters, as we have done. We suspect that, if the thermal component is similarly modeled for the stacked spectrum of Nevalainen et al. (2004), the strong nonthermal excess will disappear, perhaps entirely.

Our most suggestive result from the various stacked subsamples, that clusters hosting a radio halo or relic have the the most significant indication of a nonthermal excess on average, is also the least surprising of possible outcomes. Similarly, cooler temperature and cool core clusters have the weakest evidence for IC emission, relative to hotter and non-cool core clusters. Of course, clusters with violent mergers tend to lack cool cores and have higher temperatures, so that there is much overlap between the Radio, NCC, and Hot cluster samples. Because radio halos and relics are associated with mergers, which also produce shocks and multi-temperature gas 
distributions, the more appropriate thermal model to use might be the $2 \mathrm{~T}_{\mathrm{J}}$ model. While the T+IC models have slightly better $\chi^{2}$ values, the differences are not significant enough to suggest that a nonthermal component is required to explain the spectrum. However, we can take the best-fit normalization, which agrees with the $2 \mathrm{~T}_{\mathrm{J}} 90 \%$ upper limit, as an IC flux that can be compared with the summed diffuse radio flux of the halos and relics to derive a lower limit on the average value of $B$ for the Radio clusters. Following the IC/synchrotron theory outlined in Wik et al. (2009) using a total IC flux density at $1 \mathrm{keV}$ of $5.3 \mu \mathrm{Jy}$ and a total radio flux density of $1.385 \mathrm{Jy}$ at $1.4 \mathrm{GHz}$ (excluding relics well-outside our extraction regions, such as those in A3667 and A1367), we find $B>0.13 \mu \mathrm{G}$ for the lower limit on the average magnetic field in these clusters.

It may not be surprising that IC emission was not detected definitively in these clusters; direct measurements of cluster magnetic fields through Faraday rotation measure (RM) studies typically find line-of-sight $B$ fields on the order of several $\mu \mathrm{G}$ (Govoni \& Feretti 2004). Similar high values of $B$ are suggested by the the stability of cold fronts in merging clusters (Keshet et al. 2010), although the flow may amplify the fields in these regions. Also, RM magnetic field strengths could be biased high if stronger fields are correlated with denser gas, since RM observations are really measuring the electron density-weighted value of $B$ along the line of sight (Petrosian 2001). Such explanations, while entirely reasonable, were primarily developed to explain the lower values of $B$ implied by earlier IC detections, some of which have been more recently called into question (e.g., with Suzaku, Nakazawa et al. 2007, 2009; Wik et al. 2009). Our current sensitivity to IC emission with either pointed or survey observations can only detect nonthermal emission in clusters with radio halos if the magnetic fields are $\lesssim 0.2 \mu \mathrm{G}$. For clusters without measured radio emission, the 
even weaker field are required. Note that it is possible to observe much fainter IC emission at lower X-ray energies, and thus measure larger $B$ fields, in radio relics that are significantly displaced from the bright gas in cluster centers (Finoguenov et al. 2010).

Can the survey observations with the BAT be improved, beyond the increase in sensitivity which comes with longer accumulating exposures? Perhaps the clearest way forward is to better calibrate the spectral response of the BAT in narrower channel so that the fluxes are more reliable for steep thermal emission in the 14-24 keV energy range. At present, we may be underestimating source fluxes in these bands. If the first band is low by $\sim 2 \sigma$ and the second by $\sim 1 \sigma$, as suggested by the residuals in Figure 5.11, our nonthermal limits will increase by about $1 \sigma$ - a small but not negligible amount. The most straightforward fix is to remake the survey using the BAT's native 80 channels instead of binning them into 8 channels that are broad enough to biased by the flux calibration with the Crab. With such improved data, this study can be repeated with a sample of all the known radio halo and relic clusters to definitively detect the nonthermal excess hinted at in the stacked "Radio" subsample considered here, if it exists.

Ultimately, any IC detections, especially if marginal, will have to be confirmed by the upcoming missions with focussing hard X-ray telescopes, namely $N u S T A R^{3}$ and Astro- $H^{4}$. By resolving both contaminating point sources and the location of the hottest gas, these missions have the potential to achieve higher sensitivities than have thus far been possible.

\footnotetext{
${ }^{3}$ http://www.nustar.caltech.edu/

${ }^{4}$ http://astro-h.isas.jaxa.jp/
} 
Table 5.1. XMM-Newton Observations, Regions, and Cluster Classes

\begin{tabular}{|c|c|c|c|c|c|}
\hline Name & ObsID & $\begin{array}{c}\alpha(\mathrm{J} 2000) \\
(\mathrm{deg})\end{array}$ & $\begin{array}{c}\delta(\mathrm{J} 2000) \\
(\mathrm{deg})\end{array}$ & $\begin{array}{l}\text { Radius } \\
\text { (arcmin) }\end{array}$ & Class $^{\mathrm{a}}$ \\
\hline A0085 & 0065140101 & 10.45957 & -9.30303 & 11.6667 & $\mathrm{SCC}$ \\
\hline A0119 & 0505211001 & 14.07130 & -1.25327 & 9.3333 & $\mathrm{NCC}$ \\
\hline A0133 & 0144310101 & 15.67971 & -21.87968 & 6.7000 & $\mathrm{SCC}$ \\
\hline NGC507 & 0080540101 & 20.91068 & 33.25063 & 9.4667 & $\mathrm{SCC}$ \\
\hline A0262 & 0109980101 & 28.19002 & 36.15114 & 13.5333 & $\mathrm{SCC}$ \\
\hline A0400 & 0404010101 & 44.42226 & 6.02696 & 12.6667 & $\mathrm{NCC}$ \\
\hline A0399 & 0112260101 & 44.46513 & 13.04713 & 10.4000 & $\mathrm{NCC}$ \\
\hline A3112 & 0105660101 & 49.49456 & -44.23562 & 6.9667 & SCC \\
\hline Fornax & 0400620101 & 54.61989 & -35.45122 & 10.6333 & $\mathrm{SCC}$ \\
\hline $2 \mathrm{~A} 0335$ & 0109870101 & 54.66787 & 9.96803 & 8.9667 & $\mathrm{SCC}$ \\
\hline IIIZw54 & 0505230401 & 55.32801 & 15.40390 & 6.8667 & WCC \\
\hline A3158 & 0300211301 & 55.72316 & -53.63099 & 9.1333 & $\mathrm{NCC}$ \\
\hline NGC1550 & 0152150101 & 64.90839 & 2.40929 & 11.6667 & $\mathrm{SCC}$ \\
\hline EXO0422 & 0300210401 & 66.46339 & -8.56118 & 7.1333 & $\mathrm{SCC}$ \\
\hline A3266 & 0105260901 & 67.81198 & -61.44835 & 12.0000 & WCC \\
\hline A0496 & 0135120201 & 68.40753 & -13.26069 & 10.1667 & $\mathrm{SCC}$ \\
\hline A3376 & 0151900101 & 90.54203 & -39.95994 & 6.0000 & NCC,R \\
\hline A3391 & 0505210401 & 96.60081 & -53.69002 & 6.7333 & NCC \\
\hline A3395s & 0400010301 & 96.69188 & -54.54530 & 4.2000 & $\mathrm{NCC}$ \\
\hline A 0576 & 0205070301 & 110.35886 & 55.75948 & 9.0000 & WCC \\
\hline A0754 & 0136740101 & 137.32574 & -9.68781 & 10.1667 & NCC, R \\
\hline HydraA & 0109980301 & 139.52491 & -12.09342 & 5.0000 & NCC \\
\hline A1060 & 0206230101 & 159.17853 & -27.52841 & 8.4667 & WCC \\
\hline A1367 & 0061740101 & 176.18539 & 19.73211 & 10.0000 & NCC, R \\
\hline MKW4 & 0093060101 & 181.11522 & 1.89480 & 8.3333 & SCC \\
\hline ZwCl1215 & 0300211401 & 184.41928 & 3.65818 & 6.2333 & $\mathrm{NCC}$ \\
\hline
\end{tabular}


Table 5.1-Continued

\begin{tabular}{|c|c|c|c|c|c|}
\hline Name & ObsID & $\begin{array}{c}\alpha(\mathrm{J} 2000) \\
(\mathrm{deg})\end{array}$ & $\begin{array}{c}\delta(\mathrm{J} 2000) \\
(\mathrm{deg})\end{array}$ & $\begin{array}{l}\text { Radius } \\
\text { (arcmin) }\end{array}$ & Class $^{\mathrm{a}}$ \\
\hline NGC4636 & 0111190701 & 190.70940 & 2.69179 & 9.8333 & $\mathrm{SCC}$ \\
\hline A 3526 & 0406200101 & 192.21101 & -41.30430 & 12.9333 & $\mathrm{SCC}$ \\
\hline A1644 & 0010420201 & 194.29469 & -17.40291 & 14.7333 & $\mathrm{SCC}$ \\
\hline A1650 & 0093200101 & 194.67448 & -1.75920 & 5.1667 & WCC \\
\hline A1651 & 0203020101 & 194.84310 & -4.19633 & 7.5000 & WCC \\
\hline Coma & 0124711401 & 194.93888 & 27.95150 & 14.6667 & NCC, R \\
\hline NGC5044 & 0037950101 & 198.84908 & -16.38664 & 11.5000 & SCC \\
\hline A 3558 & 0107260101 & 202.00169 & -31.50027 & 10.2333 & WCC \\
\hline A3562 & 0105261801 & 203.40201 & -31.67382 & 6.1667 & WCC, R \\
\hline A3571 & 0086950201 & 206.86609 & -32.86052 & 8.5000 & WCC \\
\hline A1795 & 0097820101 & 207.21991 & 26.59282 & 8.0000 & $\mathrm{SCC}$ \\
\hline A3581 & 0205990101 & 211.87760 & -27.01320 & 11.0667 & $\mathrm{SCC}$ \\
\hline MKW8 & 0300210701 & 220.17560 & 3.47159 & 7.5667 & $\mathrm{NCC}$ \\
\hline A2029 & 0111270201 & 227.73326 & 5.74264 & 6.5000 & $\mathrm{SCC}$ \\
\hline A2052 & 0109920101 & 229.18501 & 7.02012 & 7.0667 & $\mathrm{SCC}$ \\
\hline MKW3S & 0109930101 & 230.45945 & 7.70323 & 9.6667 & $\mathrm{SCC}$ \\
\hline A2065 & 0112240201 & 230.62112 & 27.72063 & 6.6667 & WCC \\
\hline A2063 & 0550360101 & 230.77401 & 8.60701 & 7.1667 & WCC \\
\hline A2142 & 0111870301 & 239.56451 & 27.25178 & 6.6667 & WCC,R \\
\hline A2147 & 0505210601 & 240.56789 & 15.97177 & 11.3333 & NCC \\
\hline A2199 & 0008030201 & 247.15461 & 39.54811 & 12.3333 & $\mathrm{SCC}$ \\
\hline A2204 & 0112230301 & 248.19604 & 5.57554 & 6.1333 & SCC \\
\hline A2256 & 0141380201 & 255.96829 & 78.67197 & 8.0000 & NCC, R \\
\hline A2255 & 0112260801 & 258.22709 & 64.06428 & 8.1667 & NCC,R \\
\hline A3667 & 0206850101 & 303.16966 & -56.84081 & 13.0000 & WCC,R \\
\hline S1101 & 0123900101 & 348.49294 & -42.72664 & 6.0333 & SCC \\
\hline
\end{tabular}


Table 5.1-Continued

\begin{tabular}{lccrrr}
\hline \hline Name & ObsID & $\begin{array}{c}\alpha(\mathrm{J} 2000) \\
(\mathrm{deg})\end{array}$ & \multicolumn{1}{c}{$\delta(\mathrm{J} 2000)$} & $\begin{array}{r}\text { Radius } \\
(\mathrm{deg})\end{array}$ & \\
\hline arcmin $)$ & Class $^{\mathrm{a}}$ \\
\hline $\mathrm{A} 2589$ & 0204180101 & 350.98652 & 16.77595 & 5.0000 & WCC \\
$\mathrm{A} 2597$ & 0147330101 & 351.33334 & -12.12416 & 6.5667 & SCC \\
$\mathrm{A} 2634$ & 0002960101 & 354.62099 & 27.03107 & 11.0000 & WCC \\
$\mathrm{A} 2657$ & 0402190301 & 356.23640 & 9.19810 & 5.6667 & WCC \\
$\mathrm{A} 4038$ & 0204460101 & 356.93602 & -28.14506 & 12.3333 & WCC \\
A4059 & 0109950201 & 359.25704 & -34.75803 & 9.1333 & SCC \\
\hline
\end{tabular}

aFrom Hudson et al. (2010): SCC = "strong cool core cluster," WCC = "weak cool core cluster," and NCC = "non-cool core cluster"; clusters that host a radio halo and/or relic are labeled with "R" 
Table 5.2. EPIC-only, Single Temperature Fit Parameters

\begin{tabular}{|c|c|c|c|c|c|c|c|c|}
\hline \multirow[b]{2}{*}{ Name } & \multicolumn{4}{|c|}{ Fits $(2-12 \mathrm{keV})$} & \multicolumn{4}{|c|}{ Fits $(3-12 \mathrm{keV})$} \\
\hline & $\begin{array}{c}k T \\
(\mathrm{keV})\end{array}$ & $\begin{array}{c}\text { abund } \\
\left(\mathrm{Z}_{\odot}\right)\end{array}$ & $\begin{array}{l}\text { Norm. }^{\mathrm{a}} \\
\left(\mathrm{cm}^{-5}\right)\end{array}$ & $\chi^{2} /$ dof & $\begin{array}{c}k T \\
(\mathrm{keV})\end{array}$ & $\begin{array}{c}\text { abund } \\
\left(\mathrm{Z}_{\odot}\right)\end{array}$ & $\begin{array}{l}\text { Norm. } \\
\left(\mathrm{cm}^{-5}\right)\end{array}$ & $\chi^{2} /$ dof \\
\hline A0085 & $6.53_{-019}^{+0.20}$ & $0.355_{-0.030}^{+0.031}$ & $0.0773_{-0.0012}^{+0.0012}$ & $649.48 / 803$ & $7.30_{-0.36}^{+0.37}$ & $0.373_{-0.035}^{+0.036}$ & $0.0728_{-0.0019}^{+0.0019}$ & $395.69 / 525$ \\
\hline A0119 & $5.73_{-0.47}^{+0.48}$ & $0.227_{-0.068}^{+0.069}$ & $0.0314_{-0.0011}^{+0.0012}$ & $226.39 / 270$ & $7.18_{-1.03}^{+1.19}$ & $0.248_{-0.084}^{+0.090}$ & $0.0280_{-0.0019}^{+0.0024}$ & $110.39 / 153$ \\
\hline A0133 & $3.79_{-0.13}^{+0.14}$ & $0.446_{-0.048}^{+0.050}$ & $0.0236_{-0.0006}^{+0.0006}$ & $300.33 / 413$ & $4.30_{-0.28}^{+0.36}$ & $0.445_{-0.051}^{+0.053}$ & $0.0207_{-0.0012}^{+0.0013}$ & $129.80 / 228$ \\
\hline NGC507 & $1.50_{-0.08}^{+0.08}$ & $0.821_{-0.189}^{+0.239}$ & $0.0101_{-0.0014}^{+0.0015}$ & $132.82 / 183$ & $1.92_{-0.27}^{+0.37}$ & $0.777_{-0.393}^{+0.660}$ & $0.0071_{-0.0018}^{+0.0025}$ & $48.66 / 90$ \\
\hline A0262 & $2.23_{-0.04}^{+0.04}$ & $0.485_{-0.044}^{+0.046}$ & $0.0549_{-0.0014}^{+0.0015}$ & $584.87 / 668$ & $2.37_{-0.09}^{+0.10}$ & $0.395_{-0.053}^{+0.056}$ & $0.0548_{-0.0029}^{+0.0030}$ & $284.19 / 370$ \\
\hline A 0400 & $2.24_{-0.12}^{+0.12}$ & $0.374_{-0.098}^{+0.108}$ & $0.0206_{-0.0013}^{+0.0014}$ & $247.76 / 288$ & $2.46_{-0.26}^{+0.03}$ & $0.397_{-0.154}^{+0.183}$ & $0.0166_{-0.0026}^{+0.0029}$ & $100.91 / 150$ \\
\hline A0399 & $7.44_{-0.49}^{+0.50}$ & $0.224_{-0.053}^{+0.053}$ & $0.0356_{-0.0010}^{+0.0010}$ & $269.07 / 368$ & $8.10_{-0.77}^{+1.17}$ & $0.237_{-0.059}^{+0.062}$ & $0.0343_{-0.0018}^{+0.0018}$ & $143.88 / 220$ \\
\hline A3112 & $4.85_{-0.13}^{+0.13}$ & $0.445_{-0.029}^{+0.029}$ & $0.0365_{-0.0006}^{+0.0006}$ & $636.64 / 715$ & $5.28_{-0.22}^{+0.27}$ & $0.455_{-0.031}^{+0.032}$ & $0.0341_{-0.0012}^{+0.0012}$ & $355.09 / 439$ \\
\hline Fornax & $1.66_{-0.03}^{+0.03}$ & $0.743_{-0.065}^{+0.070}$ & $0.0191_{-0.0009}^{+0.0009}$ & $867.36 / 801$ & $2.07_{-0.14}^{+0.22}$ & $0.230_{-0.079}^{+0.089}$ & $0.0196_{-0.0020}^{+0.0023}$ & $463.80 / 494$ \\
\hline $2 \mathrm{~A} 0335$ & $3.03_{-0.06}^{+0.06}$ & $0.423_{-0.033}^{+0.034}$ & $0.1014_{-0.0019}^{+0.0019}$ & $482.66 / 649$ & $3.22_{-0.12}^{+0.13}$ & $0.395_{-0.035}^{+0.036}$ & $0.0966_{-0.0041}^{+0.0041}$ & $234.21 / 372$ \\
\hline IIIZw54 & $2.63_{-0.10}^{+0.11}$ & $0.297_{-0.058}^{+0.065}$ & $0.0198_{-0.0007}^{+0.0008}$ & $302.14 / 404$ & $3.07_{-0.24}^{+0.28}$ & $0.238_{-0.062}^{+0.067}$ & $0.0171_{-0.0015}^{+0.0016}$ & $154.89 / 219$ \\
\hline A 3158 & $5.99_{-0.35}^{+0.100}$ & $0.332_{-0.056}^{+0.057}$ & $0.0407_{-0.0012}^{+0.0012}$ & $263.15 / 351$ & $6.67_{-066}^{+0.24}$ & $0.351_{-0.064}^{+0.062}$ & $0.0376_{-0.0020}^{+0.0023}$ & $146.23 / 208$ \\
\hline NGC1550 & $1.42_{-0.04}^{+0.05}$ & $0.522_{-0.079}^{+0.090}$ & $0.0285_{-0.0022}^{+0.0023}$ & $198.84 / 263$ & $1.60_{-0.15}^{+0.19}$ & $0.281_{-0.158}^{+0.0204}$ & $0.0292_{-0.0060}^{+0.0076}$ & $78.02 / 119$ \\
\hline EXO0422 & $3.06_{-0.07}^{+0.04}$ & $0.357_{-0.032}^{+0.033}$ & $0.0304_{-0.0006}^{+0.0006}$ & $597.13 / 744$ & $3.23_{-0.13}^{+0.15}$ & $0.337_{-0.034}^{+0.036}$ & $0.0284_{-0.0013}^{+0.0013}$ & $318.60 / 437$ \\
\hline A3266 & $8.31_{-0.28}^{+0.09}$ & $0.196_{-0.030}^{+0.030}$ & $0.0724_{-0.0010}^{+0.0010}$ & $830.96 / 1051$ & $8.56_{-0.44}^{+0.13}$ & $0.197_{-0.032}^{+0.034}$ & $0.0716_{-0.0022}^{+0.0019}$ & $559.53 / 721$ \\
\hline A0496 & $4.36_{-0.10}^{+0.08}$ & $0.394_{-0.021}^{+0.021}$ & $0.0835_{-0.0010}^{+0.0012}$ & $1003.00 / 1083$ & $4.68_{-0.14}^{+0.14}$ & $0.388_{-0.022}^{+0.022}$ & $0.0790_{-0.0019}^{+0.0019}$ & $610.07 / 757$ \\
\hline
\end{tabular}


Table 5.2-Continued

\begin{tabular}{|c|c|c|c|c|c|c|c|c|}
\hline \multirow[b]{2}{*}{ Name } & \multicolumn{4}{|c|}{ Fits $(2-12 \mathrm{keV})$} & \multicolumn{4}{|c|}{ Fits $(3-12 \mathrm{keV})$} \\
\hline & $\begin{array}{c}k T \\
(\mathrm{keV})\end{array}$ & $\begin{array}{l}\text { abund } \\
\left(\mathrm{Z}_{\odot}\right)\end{array}$ & $\begin{array}{l}\text { Norm. } \\
\left(\mathrm{cm}^{-5}\right)\end{array}$ & $\chi^{2} /$ dof & $\begin{array}{c}k T \\
(\mathrm{keV})\end{array}$ & $\begin{array}{c}\text { abund } \\
\left(\mathrm{Z}_{\odot}\right)\end{array}$ & $\begin{array}{l}\text { Norm. } \\
\left(\mathrm{cm}^{-5}\right)\end{array}$ & $\chi^{2} /$ dof \\
\hline A3376 & $4.00_{-0.27}^{+0.29}$ & $0.498_{-0.118}^{+0.126}$ & $0.0108_{-0.0005}^{+0.0005}$ & $129.58 / 167$ & $5.76_{-0.94}^{+1.15}$ & $0.454_{-0.130}^{+0.146}$ & $0.0085_{-0.0008}^{+0.0010}$ & $52.36 / 75$ \\
\hline A3391 & $6.45_{-0.31}^{+0.33}$ & $0.312_{-0.049}^{+0.050}$ & $0.0207_{-0.0005}^{+0.0005}$ & $371.79 / 482$ & $6.85_{-0.49}^{+0.58}$ & $0.315_{-0.052}^{+0.130}$ & $0.0200_{-0.0008}^{+0.0008}$ & $199.48 / 294$ \\
\hline A3395s & $5.76_{-0.66}^{+0.66}$ & $0.248_{-0.099}^{+0.102}$ & $0.0077_{-0.0004}^{+0.0004}$ & $113.23 / 205$ & $5.95_{-1.13}^{+1.30}$ & $0.246_{-0.104}^{+0.112}$ & $0.0075_{-0.0008}^{+0.0011}$ & $52.46 / 115$ \\
\hline A0576 & $4.06_{-0.26}^{+0.28}$ & $0.377_{-0.083}^{+0.087}$ & $0.0245_{-0.0011}^{+0.0012}$ & $167.36 / 217$ & $4.29_{-0.47}^{+0.68}$ & $0.378_{-0.087}^{+0.094}$ & $0.0228_{-0.0023}^{+0.0025}$ & $81.90 / 120$ \\
\hline A0754 & $9.16_{-0.37}^{+0.38}$ & $0.281_{-0.032}^{+0.032}$ & $0.0703_{-0.0008}^{+0.0008}$ & $780.81 / 951$ & $9.43_{-0.54}^{+0.55}$ & $0.285_{-0.033}^{+0.034}$ & $0.0697_{-0.0014}^{+0.0015}$ & $523.22 / 636$ \\
\hline HydraA & $3.98_{-0.09}^{+0.09}$ & $0.286_{-0.025}^{+0.026}$ & $0.0452_{-0.0008}^{+0.0008}$ & $607.64 / 709$ & $4.39_{-0.18}^{+0.19}$ & $0.282_{-0.026}^{+0.026}$ & $0.0412_{-0.0014}^{+0.0015}$ & $329.19 / 434$ \\
\hline A1060 & $3.20_{-0.05}^{+0.05}$ & $0.406_{-0.023}^{+0.024}$ & $0.0592_{-0.0008}^{+0.0008}$ & $853.00 / 963$ & $3.44_{-0.09}^{+0.10}$ & $0.384_{-0.024}^{+0.024}$ & $0.0544_{-0.0014}^{+0.0015}$ & $498.30 / 632$ \\
\hline A1367 & $3.79_{-0.12}^{+0.12}$ & $0.297_{-0.036}^{+0.037}$ & $0.0327_{-0.0007}^{+0.0007}$ & $472.97 / 594$ & $4.18_{-0.23}^{+0.25}$ & $0.292_{-0.038}^{+0.039}$ & $0.0302_{-0.0014}^{+0.0016}$ & $250.18 / 335$ \\
\hline MKW4 & $1.69_{-0.11}^{+0.12}$ & $0.660_{-0.190}^{+0.248}$ & $0.0145_{-0.0021}^{+0.0024}$ & $46.08 / 97$ & $1.75_{-0.26}^{+0.29}$ & $0.914_{-0.527}^{+1.098}$ & $0.0119_{-0.0040}^{+0.0062}$ & $18.35 / 39$ \\
\hline ZwCl1215 & $7.15_{-0.34}^{+0.35}$ & $0.283_{-0.037}^{+0.038}$ & $0.0257_{-0.0005}^{+0.0005}$ & $455.11 / 603$ & $7.66_{-0.52}^{+0.53}$ & $0.300_{-0.042}^{+0.044}$ & $0.0248_{-0.0000}^{+0.0009}$ & $269.38 / 363$ \\
\hline NGC4636 & $0.95_{-0.08}^{+0.11}$ & $0.848_{-0.255}^{+0.478}$ & $0.0060_{-0.0015}^{+0.0016}$ & $227.15 / 354$ & $3.44_{-1.88}^{+4.08}$ & $0.000_{-0.000}^{+1.498}$ & $0.0019_{-0.0010}^{+0.0008}$ & $95.54 / 145$ \\
\hline A3526 & $3.90_{-0.02}^{+0.06}$ & $0.539_{-0.013}^{+0.010}$ & $0.1095_{-0.0017}^{+0.0018}$ & $3524.37 / 2257$ & $3.66_{-0.03}^{+0.05}$ & $0.570_{-0.010}^{+0.010}$ & $0.1243_{-0.0013}^{+0.0007}$ & $2474.07 / 1925$ \\
\hline A1644 & $5.12_{-0.23}^{+0.24}$ & $0.294_{-0.045}^{+0.040}$ & $0.0443_{-0.0012}^{+0.0012}$ & $389.51 / 525$ & $5.74_{-0.56}^{+0.52}$ & $0.306_{-0.050}^{+0.052}$ & $0.0412_{-0.0021}^{+0.0027}$ & $227.60 / 296$ \\
\hline A1650 & $5.96_{-0.17}^{+0.17}$ & $0.393_{-0.026}^{+0.045}$ & $0.0275_{-0.0004}^{+0.0004}$ & $748.85 / 910$ & $6.13_{-0.25}^{+0.26}$ & $0.396_{-0.028}^{+0.028}$ & $0.0271_{-0.0007}^{+0.0007}$ & $449.63 / 594$ \\
\hline A1651 & $6.43_{-0.35}^{+0.37}$ & $0.389_{-0.056}^{+0.020}$ & $0.0348_{-0.0011}^{+0.0011}$ & $197.37 / 326$ & $6.82_{-0.56}^{+0.75}$ & $0.405_{-0.061}^{+0.028}$ & $0.0338_{-0.0019}^{+0.0021}$ & $118.80 / 190$ \\
\hline Coma & $8.53_{-0.13}^{+0.19}$ & $0.248_{-0.015}^{+0.015}$ & $0.2443_{-0.0016}^{+0.0016}$ & $1787.94 / 2158$ & $8.65_{-0.22}^{+0.26}$ & $0.249_{-0.015}^{+0.001}$ & $0.2439_{-0.0025}^{+0.0030}$ & $1445.12 / 1826$ \\
\hline
\end{tabular}


Table 5.2-Continued

\begin{tabular}{|c|c|c|c|c|c|c|c|c|}
\hline \multirow[b]{2}{*}{ Name } & \multicolumn{4}{|c|}{ Fits $(2-12 \mathrm{keV})$} & \multicolumn{4}{|c|}{ Fits $(3-12 \mathrm{keV})$} \\
\hline & $\begin{array}{c}k T \\
(\mathrm{keV})\end{array}$ & $\begin{array}{c}\text { abund } \\
\left(\mathrm{Z}_{\odot}\right)\end{array}$ & $\begin{array}{l}\text { Norm. }^{\mathrm{a}} \\
\left(\mathrm{cm}^{-5}\right)\end{array}$ & $\chi^{2} /$ dof & $\begin{array}{c}k T \\
(\mathrm{keV})\end{array}$ & $\begin{array}{c}\text { abund } \\
\left(\mathrm{Z}_{\odot}\right)\end{array}$ & $\begin{array}{l}\text { Norm. } \\
\left(\mathrm{cm}^{-5}\right)\end{array}$ & $\chi^{2} /$ dof \\
\hline NGC5044 & $1.21_{-0.04}^{+0.04}$ & $0.797_{-0.124}^{+0.148}$ & $0.0247_{-0.0027}^{+0.0029}$ & $393.86 / 488$ & $1.49_{-0.14}^{+0.19}$ & $0.627_{-0.294}^{+0.461}$ & $0.0191_{-0.0047}^{+0.0061}$ & $176.36 / 229$ \\
\hline A3558 & $5.92_{-0.10}^{+0.10}$ & $0.323_{-0.015}^{+0.015}$ & $0.0665_{-0.0005}^{+0.0005}$ & $1271.71 / 1456$ & $6.25_{-0.15}^{+0.15}$ & $0.334_{-0.016}^{+0.016}$ & $0.0641_{-0.0010}^{+0.0010}$ & $904.58 / 1124$ \\
\hline A3562 & $5.09_{-0.55}^{+0.65}$ & $0.417_{-0.146}^{+0.155}$ & $0.0175_{-0.0012}^{+0.0013}$ & $54.45 / 125$ & $5.69_{-1.09}^{+1.52}$ & $0.416_{-0.153}^{+0.173}$ & $0.0163_{-0.0022}^{+0.0028}$ & $24.75 / 69$ \\
\hline A3571 & $7.24_{-0.15}^{+0.15}$ & $0.372_{-0.019}^{+0.019}$ & $0.1104_{-0.0011}^{+0.0011}$ & $1610.57 / 1874$ & $7.57_{-0.21}^{+0.21}$ & $0.385_{-0.021}^{+0.021}$ & $0.1074_{-0.0017}^{+0.0017}$ & $1260.22 / 1542$ \\
\hline A1795 & $5.67_{-0.08}^{+0.08}$ & $0.369_{-0.013}^{+0.013}$ & $0.0797_{-0.0006}^{+0.0006}$ & $1673.53 / 1907$ & $5.89_{-0.12}^{+0.12}$ & $0.375_{-0.014}^{+0.014}$ & $0.0781_{-0.0011}^{+0.0011}$ & $1337.50 / 1575$ \\
\hline A3581 & $1.91_{-0.04}^{+0.04}$ & $0.556_{-0.055}^{+0.059}$ & $0.0271_{-0.0010}^{+0.0010}$ & $431.36 / 546$ & $2.04_{-0.10}^{+0.12}$ & $0.404_{-0.074}^{+0.082}$ & $0.0275_{-0.0023}^{+0.0024}$ & $198.57 / 278$ \\
\hline MKW8 & $3.36_{-0.21}^{+0.30}$ & $0.350_{-0.092}^{+0.099}$ & $0.0135_{-0.0007}^{+0.0007}$ & $150.85 / 221$ & $3.88_{-0.48}^{+0.63}$ & $0.321_{-0.097}^{+0.104}$ & $0.0119_{-0.0014}^{+0.0014}$ & $69.64 / 120$ \\
\hline A2029 & $7.97_{-0.22}^{+0.22}$ & $0.428_{-0.029}^{+0.029}$ & $0.0782_{-0.0010}^{+0.0010}$ & $864.77 / 943$ & $8.46_{-0.30}^{+0.39}$ & $0.453_{-0.033}^{+0.034}$ & $0.0754_{-0.0016}^{+0.0016}$ & $539.03 / 632$ \\
\hline A2052 & $3.01_{-0.05}^{+0.05}$ & $0.500_{-0.029}^{+0.029}$ & $0.0480_{-0.0007}^{+0.0007}$ & $717.07 / 849$ & $3.22_{-0.09}^{+0.10}$ & $0.471_{-0.031}^{+0.031}$ & $0.0454_{-0.0015}^{+0.0015}$ & $426.04 / 523$ \\
\hline MKW3S & $3.36_{-0.06}^{+0.06}$ & $0.388_{-0.026}^{+0.027}$ & $0.0392_{-0.0006}^{+0.0006}$ & $693.59 / 838$ & $3.65_{-0.12}^{+0.13}$ & $0.385_{-0.028}^{+0.028}$ & $0.0361_{-0.0012}^{+0.0012}$ & $398.37 / 515$ \\
\hline A2065 & $6.51_{-0.49}^{+0.60}$ & $0.261_{-0.077}^{+0.070}$ & $0.0290_{-0.0018}^{+0.0018}$ & $161.22 / 249$ & $6.76_{-0.83}^{+1.14}$ & $0.260_{-0.080}^{+0.082}$ & $0.0282_{-0.0031}^{+0.0034}$ & $95.15 / 156$ \\
\hline A2063 & $4.34_{-0.13}^{+0.14}$ & $0.345_{-0.032}^{+0.034}$ & $0.0371_{-0.0007}^{+0.0008}$ & $640.80 / 774$ & $4.55_{-0.22}^{+0.23}$ & $0.344_{-0.033}^{+0.034}$ & $0.0356_{-0.0013}^{+0.0014}$ & $419.72 / 510$ \\
\hline $\mathrm{A} 2142$ & $9.68_{-1.88}^{+2.83}$ & $0.280_{-0.221}^{+0.216}$ & $0.0642_{-0.0051}^{+0.0052}$ & $273.45 / 157$ & $8.01_{-1.96}^{+4.38}$ & $0.257_{-0.182}^{+0.189}$ & $0.0681_{-0.0103}^{+0.0116}$ & $131.32 / 93$ \\
\hline $\mathrm{A} 2147$ & $5.17_{-0.43}^{+0.58}$ & $0.238_{-0.096}^{+0.100}$ & $0.0410_{-0.0023}^{+0.0024}$ & $164.44 / 220$ & $6.46_{-1.06}^{+1.48}$ & $0.249_{-0.113}^{+0.120}$ & $0.0341_{-0.0037}^{+0.0041}$ & $82.91 / 129$ \\
\hline A2199 & $4.45_{-0.09}^{+0.45}$ & $0.363_{-0.020}^{+0.021}$ & $0.1021_{-0.0012}^{+0.0012}$ & $910.01 / 1069$ & $4.59_{-0.14}^{+0.14}$ & $0.366_{-0.021}^{+0.022}$ & $0.0999_{-0.0023}^{+0.0023}$ & $565.55 / 737$ \\
\hline A 2204 & $7.11_{-0.23}^{+0.24}$ & $0.397_{-0.028}^{+0.029}$ & $0.0468_{-0.0007}^{+0.0007}$ & $618.61 / 772$ & $7.46_{-0.32}^{+0.34}$ & $0.413_{-0.032}^{+0.033}$ & $0.0456_{-0.0012}^{+0.0012}$ & $365.71 / 498$ \\
\hline
\end{tabular}


Table 5.2-Continued

\begin{tabular}{|c|c|c|c|c|c|c|c|c|}
\hline \multirow[b]{2}{*}{ Name } & \multicolumn{4}{|c|}{ Fits $(2-12 \mathrm{keV})$} & \multicolumn{4}{|c|}{ Fits $(3-12 \mathrm{keV})$} \\
\hline & $\begin{array}{c}k T \\
(\mathrm{keV})\end{array}$ & $\begin{array}{l}\text { abund } \\
\left(\mathrm{Z}_{\odot}\right)\end{array}$ & $\begin{array}{l}\text { Norm. } \\
\left(\mathrm{cm}^{-5}\right)\end{array}$ & $\chi^{2} /$ dof & $\begin{array}{c}k T \\
(\mathrm{keV})\end{array}$ & $\begin{array}{c}\text { abund } \\
\left(\mathrm{Z}_{\odot}\right)\end{array}$ & $\begin{array}{l}\text { Norm. }^{\mathrm{a}} \\
\left(\mathrm{cm}^{-5}\right)\end{array}$ & $\chi^{2} /$ dof \\
\hline A2256 & $6.97_{-0.39}^{+0.40}$ & $0.299_{-0.043}^{+0.044}$ & $0.0530_{-0.0011}^{+0.0013}$ & $324.79 / 434$ & $8.07_{-0.59}^{+0.67}$ & $0.338_{-0.053}^{+0.056}$ & $0.0491_{-0.0018}^{+0.0019}$ & $176.12 / 253$ \\
\hline $\mathrm{A} 2255$ & $7.81_{-0.87}^{+0.95}$ & $0.267_{-0.107}^{+0.110}$ & $0.0237_{-0.0012}^{+0.0011}$ & $96.21 / 184$ & $8.10_{-1.35}^{+1.69}$ & $0.255_{-0.107}^{+0.118}$ & $0.0235_{-0.0020}^{+0.0023}$ & $48.15 / 110$ \\
\hline A3667 & $6.62_{-0.11}^{+0.81}$ & $0.266_{-0.015}^{+0.015}$ & $0.0761_{-0.0006}^{+0.0006}$ & $1495.08 / 1643$ & $7.20_{-0.21}^{+0.35}$ & $0.277_{-0.017}^{+0.017}$ & $0.0728_{-0.0010}^{+0.0010}$ & $1141.52 / 1311$ \\
\hline S1101 & $2.65_{-0.06}^{+0.06}$ & $0.337_{-0.037}^{+0.038}$ & $0.0259_{-0.0007}^{+0.0007}$ & $412.72 / 525$ & $2.86_{-0.13}^{+0.14}$ & $0.336_{-0.042}^{+0.044}$ & $0.0235_{-0.0013}^{+0.0013}$ & $208.02 / 274$ \\
\hline A 2589 & $3.69_{-0.12}^{+0.13}$ & $0.542_{-0.050}^{+0.052}$ & $0.0205_{-0.0005}^{+0.000}$ & $326.99 / 437$ & $3.87_{-0.21}^{+0.23}$ & $0.545_{-0.053}^{+0.055}$ & $0.0197_{-0.0010}^{+0.0010}$ & $155.20 / 243$ \\
\hline A 2597 & $3.34_{-0.06}^{+0.07}$ & $0.334_{-0.024}^{+0.025}$ & $0.0273_{-0.0005}^{+0.0005}$ & $610.98 / 712$ & $3.91_{-0.16}^{+0.17}$ & $0.314_{-0.025}^{+0.025}$ & $0.0236_{-0.0009}^{+0.0009}$ & $300.35 / 398$ \\
\hline $\mathrm{A} 2634$ & $4.56_{-0.48}^{+0.57}$ & $0.269_{-0.126}^{+0.133}$ & $0.0184_{-0.0012}^{+0.0013}$ & $96.50 / 131$ & $4.90_{-0.94}^{+1.36}$ & $0.269_{-0.135}^{+0.143}$ & $0.0177_{-0.0026}^{+0.0032}$ & $51.97 / 70$ \\
\hline A 2657 & $5.16_{-0.29}^{+0.48}$ & $0.283_{-0.063}^{+0.065}$ & $0.0256_{-0.0015}^{+0.0015}$ & $268.87 / 347$ & $5.88_{-0.65}^{+0.69}$ & $0.251_{-0.067}^{+0.069}$ & $0.0233_{-0.0024}^{+0.0025}$ & $170.68 / 226$ \\
\hline A 4038 & $3.20_{-0.05}^{+0.05}$ & $0.365_{-0.024}^{+0.024}$ & $0.0596_{-0.0009}^{+0.0009}$ & $870.70 / 1049$ & $3.42_{-0.11}^{+0.12}$ & $0.343_{-0.025}^{+0.026}$ & $0.0558_{-0.0017}^{+0.000}$ & $577.41 / 717$ \\
\hline A4059 & $4.24_{-0.12}^{+0.14}$ & $0.425_{-0.035}^{+0.036}$ & $0.0342_{-0.0007}^{+0.0007}$ & $471.59 / 685$ & $4.48_{-0.22}^{+0.23}$ & $0.425_{-0.036}^{+0.037}$ & $0.0329_{-0.0012}^{+0.0013}$ & $251.18 / 418$ \\
\hline
\end{tabular}

${ }^{a}$ Normalization of the APEC thermal spectrum, which is given by $\left\{10^{-14} /\left[4 \pi(1+z)^{2} D_{A}^{2}\right]\right\} \int n_{e} n_{H} d V$, where $z$ is the redshift, $D_{A}$ is the angular diameter distance, $n_{e}$ is the electron density, $n_{H}$ is the ionized hydrogen density, and $V$ is the volume of the cluster. 
Table 5.3. Joint Thermal and Nonthermal Fits to the EPIC and BAT Spectra $(2-195 \mathrm{keV})$

\begin{tabular}{|c|c|c|c|c|c|c|c|}
\hline \multirow[b]{2}{*}{ Name } & \multirow[b]{2}{*}{ Model $^{\mathrm{a}}$} & \multicolumn{3}{|c|}{ Component 1} & \multicolumn{2}{|c|}{ Component 2} & \multirow[b]{2}{*}{$\chi^{2} /$ dof } \\
\hline & & $\begin{array}{c}k T \\
(\mathrm{keV})\end{array}$ & $\begin{array}{c}\text { abund } \\
\mathrm{Z}_{\odot}\end{array}$ & $\begin{array}{l}\text { Norm. }^{b} \\
\left(\mathrm{~cm}^{-5}\right)\end{array}$ & $\begin{array}{c}k T \\
(\mathrm{keV})\end{array}$ & Norm. $^{\mathrm{c}}$ & \\
\hline \multirow[t]{3}{*}{ A0085 } & $1 \mathrm{~T}$ & $6.46_{-0.18}^{+0.19}$ & $0.358_{-0.030}^{+0.031}$ & $0.0775_{-0.0010}^{+0.0010}$ & \multirow{3}{*}{8.64} & & $654.76 / 812$ \\
\hline & $2 \mathrm{~T}$ & 5.45 & 0.371 & 0.0497 & & 0.0282 & $651.63 / 810$ \\
\hline & $\mathrm{T}+\mathrm{IC}$ & $6.48_{-0.19}^{+0.19}$ & $0.361_{-0.035}^{+0.036}$ & $0.0760_{-0.0042}^{+0.0026}$ & & $<0.0019$ & $654.12 / 811$ \\
\hline \multirow[t]{3}{*}{ A0119 } & $1 \mathrm{~T}$ & $5.71_{-0.45}^{+0.46}$ & $0.227_{-0.068}^{+0.069}$ & $0.0316_{-0.0010}^{+0.0011}$ & \multirow{3}{*}{8.53} & & $231.08 / 279$ \\
\hline & $2 \mathrm{~T}$ & 4.33 & 0.245 & 0.0200 & & 0.0123 & $229.57 / 277$ \\
\hline & $\mathrm{T}+\mathrm{IC}$ & $5.68_{-0.48}^{+0.49}$ & $0.240_{-0.074}^{+0.078}$ & $0.0298_{-0.0042}^{+0.0027}$ & & $<0.0019$ & $230.53 / 278$ \\
\hline \multirow[t]{3}{*}{ A0133 } & $1 \mathrm{~T}$ & $3.78_{-0.13}^{+0.14}$ & $0.446_{-0.048}^{+0.050}$ & $0.0236_{-0.0006}^{+0.0006}$ & \multirow{4}{*}{4.33} & & $302.63 / 422$ \\
\hline & $2 \mathrm{~T}$ & 0.65 & 0.452 & 0.0148 & & 0.0206 & $285.60 / 420$ \\
\hline & $\mathrm{T}+\mathrm{IC}$ & $3.36_{-0.20}^{+0.39}$ & $0.636_{-0.173}^{+0.150}$ & $0.0176_{-0.0025}^{+0.0046}$ & & $0.0018_{-0.0013}^{+0.0007}$ & $297.32 / 421$ \\
\hline \multirow[t]{3}{*}{ NGC507 } & $1 \mathrm{~T}$ & $1.48_{-0.08}^{+0.08}$ & $0.832_{-0.195}^{+0.250}$ & $0.0100_{-0.0014}^{+0.0015}$ & & & $144.35 / 192$ \\
\hline & $2 \mathrm{~T}$ & 1.26 & 0.872 & 0.0095 & \multirow[t]{2}{*}{6.07} & 0.0009 & $136.35 / 190$ \\
\hline & $\mathrm{T}+\mathrm{IC}$ & $1.29_{-0.12}^{+0.15}$ & $0.984_{-0.302}^{+0.457}$ & $0.0084_{-0.0021}^{+0.0021}$ & & $0.0004_{-0.0002}^{+0.0002}$ & $137.09 / 191$ \\
\hline \multirow[t]{3}{*}{ A0262 } & $1 \mathrm{~T}$ & $2.20_{-0.04}^{+0.04}$ & $0.485_{-0.044}^{+0.046}$ & $0.0545_{-0.0014}^{+0.0014}$ & \multirow{3}{*}{4.64} & & $591.81 / 677$ \\
\hline & $2 \mathrm{~T}$ & 2.00 & 0.482 & 0.0508 & & 0.0052 & $588.01 / 675$ \\
\hline & $\mathrm{T}+\mathrm{IC}$ & $2.11_{-0.03}^{+0.09}$ & $0.521_{-0.052}^{+0.056}$ & $0.0519_{-0.0025}^{+0.0026}$ & & $0.0008_{-0.0007}^{+0.0006}$ & $588.14 / 676$ \\
\hline \multirow[t]{3}{*}{ A0400 } & $1 \mathrm{~T}$ & $2.24_{-0.11}^{+0.12}$ & $0.366_{-0.097}^{+0.107}$ & $0.0207_{-0.0013}^{+0.0014}$ & \multirow{4}{*}{2.30} & & $265.30 / 297$ \\
\hline & $2 \mathrm{~T}$ & 2.18 & 0.368 & 0.0110 & & 0.0096 & $265.48 / 295$ \\
\hline & $\mathrm{T}+\mathrm{IC}$ & $2.23_{-0.08}^{+0.13}$ & $0.357_{-0.089}^{+0.117}$ & $0.0208_{-0.0010}^{+0.0012}$ & & $<0.0005$ & $265.30 / 296$ \\
\hline \multirow[t]{3}{*}{ A0399 } & $1 \mathrm{~T}$ & $7.28_{-0.45}^{+0.08}$ & $0.224_{-0.052}^{+0.089}$ & $0.0357_{-0.0007}^{+0.0010}$ & & & $276.34 / 377$ \\
\hline & $2 \mathrm{~T}$ & 7.37 & 0.224 & 0.0108 & \multirow[t]{3}{*}{7.24} & 0.0249 & $276.35 / 375$ \\
\hline & $\mathrm{T}+\mathrm{IC}$ & $7.29_{-0.46}^{+0.46}$ & $0.224_{-0.052}^{+0.052}$ & $0.0357_{-0.0027}^{+0.0008}$ & & $<0.0009$ & $276.35 / 376$ \\
\hline \multirow[t]{3}{*}{ A3112 } & $1 \mathrm{~T}$ & $4.84_{-0.13}^{+0.13}$ & $0.447_{-0.029}^{+0.030}$ & $0.0365_{-0.0005}^{+0.0006}$ & & & $655.48 / 724$ \\
\hline & $2 \mathrm{~T}$ & 2.08 & 0.495 & 0.0097 & \multirow[t]{2}{*}{5.84} & 0.0284 & $635.35 / 722$ \\
\hline & $\mathrm{T}+\mathrm{IC}$ & $4.64_{-0.17}^{+0.19}$ & $0.563_{-0.071}^{+0.070}$ & $0.0286_{-0.0028}^{+0.0035}$ & & $0.0024_{-0.0010}^{+0.0008}$ & $638.06 / 723$ \\
\hline \multirow[t]{3}{*}{ Fornax } & $1 \mathrm{~T}$ & $1.64_{-0.03}^{+0.03}$ & $0.748_{-0.066}^{+0.071}$ & $0.0188_{-0.0009}^{+0.0009}$ & \multirow{3}{*}{20.56} & & $847.93 / 810$ \\
\hline & $2 \mathrm{~T}$ & 1.43 & 0.796 & 0.0175 & & 0.0016 & $777.31 / 808$ \\
\hline & $\mathrm{T}+\mathrm{IC}$ & $1.41_{-0.05}^{+0.05}$ & $1.038_{-0.133}^{+0.163}$ & $0.0134_{-0.0014}^{+0.0014}$ & & $0.0011_{-0.0002}^{+0.0002}$ & $759.77 / 809$ \\
\hline
\end{tabular}


Table 5.3-Continued

\begin{tabular}{|c|c|c|c|c|c|c|c|}
\hline \multirow[b]{2}{*}{ Name } & \multirow[b]{2}{*}{ Model $^{\mathrm{a}}$} & \multicolumn{3}{|c|}{ Component 1} & \multicolumn{2}{|c|}{ Component 2} & \multirow[b]{2}{*}{$\chi^{2} /$ dof } \\
\hline & & $\begin{array}{c}k T \\
(\mathrm{keV})\end{array}$ & $\begin{array}{c}\text { abund } \\
\mathrm{Z}_{\odot}\end{array}$ & $\begin{array}{l}\text { Norm. }^{\mathrm{b}} \\
\left(\mathrm{cm}^{-5}\right)\end{array}$ & $\begin{array}{c}k T \\
(\mathrm{keV})\end{array}$ & Norm. ${ }^{\mathrm{c}}$ & \\
\hline \multirow[t]{3}{*}{$2 \mathrm{~A} 0335$} & $1 \mathrm{~T}$ & $3.03_{-0.06}^{+0.06}$ & $0.424_{-0.033}^{+0.034}$ & $0.0999_{-0.0017}^{+0.0017}$ & \multirow{3}{*}{3.31} & & $506.39 / 658$ \\
\hline & $2 \mathrm{~T}$ & 2.78 & 0.424 & 0.0524 & & 0.0475 & $506.39 / 656$ \\
\hline & $\mathrm{T}+\mathrm{IC}$ & $2.98_{-0.09}^{+0.09}$ & $0.441_{-0.042}^{+0.045}$ & $0.0973_{-0.0044}^{+0.0038}$ & & $<0.0020$ & $503.87 / 657$ \\
\hline \multirow[t]{3}{*}{ IIIZw54 } & $1 \mathrm{~T}$ & $2.62_{-0.10}^{+0.10}$ & $0.299_{-0.059}^{+0.062}$ & $0.0198_{-0.0007}^{+0.0008}$ & \multirow{3}{*}{2.63} & & $303.78 / 413$ \\
\hline & $2 \mathrm{~T}$ & 2.60 & 0.300 & 0.0103 & & 0.0095 & $303.73 / 411$ \\
\hline & $\mathrm{T}+\mathrm{IC}$ & $2.22_{-0.18}^{+0.29}$ & $0.404_{-0.107}^{+0.093}$ & $0.0161_{-0.0016}^{+0.0026}$ & & $0.0012_{-0.0008}^{+0.0004}$ & $297.93 / 412$ \\
\hline \multirow[t]{3}{*}{ A3158 } & $1 \mathrm{~T}$ & $5.89_{-0.33}^{+0.35}$ & $0.333_{-0.056}^{+0.057}$ & $0.0406_{-0.0010}^{+0.0010}$ & \multirow{3}{*}{6.19} & & $270.08 / 360$ \\
\hline & $2 \mathrm{~T}$ & 0.71 & 0.345 & 0.0080 & & 0.0393 & $267.08 / 358$ \\
\hline & $\mathrm{T}+\mathrm{IC}$ & $5.92_{-0.34}^{+0.35}$ & $0.338_{-0.060}^{+0.064}$ & $0.0398_{-0.0034}^{+0.0018}$ & & $<0.0014$ & $269.85 / 359$ \\
\hline \multirow[t]{3}{*}{ NGC1550 } & $1 \mathrm{~T}$ & $1.42_{-0.04}^{+0.05}$ & $0.524_{-0.080}^{+0.092}$ & $0.0288_{-0.0022}^{+0.0024}$ & \multirow{4}{*}{1.57} & & $207.02 / 272$ \\
\hline & $2 \mathrm{~T}$ & 1.30 & 0.533 & 0.0173 & & 0.0118 & $206.93 / 270$ \\
\hline & $\mathrm{T}+\mathrm{IC}$ & $1.34_{-0.08}^{+0.08}$ & $0.547_{-0.087}^{+0.104}$ & $0.0278_{-0.0027}^{+0.0029}$ & & $0.0004_{-0.0003}^{+0.0003}$ & $202.69 / 271$ \\
\hline \multirow[t]{3}{*}{ EXO0422 } & $1 \mathrm{~T}$ & $3.05_{-0.07}^{+0.07}$ & $0.359_{-0.032}^{+0.033}$ & $0.0303_{-0.0005}^{+0.0005}$ & & & $604.99 / 753$ \\
\hline & $2 \mathrm{~T}$ & 2.17 & 0.363 & 0.0144 & \multirow[t]{2}{*}{3.84} & 0.0168 & $600.84 / 751$ \\
\hline & $\mathrm{T}+\mathrm{IC}$ & $2.76_{-0.13}^{+0.23}$ & $0.465_{-0.093}^{+0.069}$ & $0.0255_{-0.0018}^{+0.0035}$ & & $0.0014_{-0.0010}^{+0.0005}$ & $598.33 / 752$ \\
\hline \multirow[t]{3}{*}{ A 3266} & $1 \mathrm{~T}$ & $8.41_{-0.26}^{+0.33}$ & $0.193_{-0.030}^{+0.031}$ & $0.0722_{-0.0008}^{+0.0008}$ & \multirow{3}{*}{9.51} & & $837.81 / 1060$ \\
\hline & $2 \mathrm{~T}$ & 4.45 & 0.203 & 0.0119 & & 0.0611 & $834.47 / 1058$ \\
\hline & $\mathrm{T}+\mathrm{IC}$ & $8.47_{-0.28}^{+0.37}$ & $0.212_{-0.034}^{+0.033}$ & $0.0672_{-0.0037}^{+0.0032}$ & & $0.0017_{-0.0011}^{+0.0012}$ & $830.76 / 1059$ \\
\hline \multirow[t]{3}{*}{ A0496 } & $1 \mathrm{~T}$ & $4.34_{-0.10}^{+0.08}$ & $0.397_{-0.021}^{+0.023}$ & $0.0835_{-0.0008}^{+0.00011}$ & \multirow{4}{*}{6.83} & & $1011.90 / 1092$ \\
\hline & $2 \mathrm{~T}$ & 3.23 & 0.451 & 0.0555 & & 0.0302 & $999.75 / 1090$ \\
\hline & $\mathrm{T}+\mathrm{IC}$ & $4.28_{-0.08}^{+0.13}$ & $0.414_{-0.033}^{+0.021}$ & $0.0810_{-0.0032}^{+0.0034}$ & & $<0.0019$ & $1011.04 / 1091$ \\
\hline \multirow[t]{3}{*}{ A 3376} & $1 \mathrm{~T}$ & $4.00_{-0.27}^{+0.29}$ & $0.497_{-0.118}^{+0.126}$ & $0.0108_{-0.0005}^{+0.0005}$ & & & $140.94 / 176$ \\
\hline & $2 \mathrm{~T}$ & 2.08 & 0.579 & 0.0061 & \multirow[t]{3}{*}{7.56} & 0.0054 & $130.31 / 174$ \\
\hline & $\mathrm{T}+\mathrm{IC}$ & $3.06_{-0.47}^{+0.60}$ & $1.318_{-0.578}^{+1.679}$ & $0.0045_{-0.0024}^{+0.0026}$ & & $0.0018_{-0.0008}^{+0.0006}$ & $130.58 / 175$ \\
\hline \multirow[t]{3}{*}{ A3391 } & $1 \mathrm{~T}$ & $6.45_{-0.31}^{+0.31}$ & $0.310_{-0.049}^{+0.050}$ & $0.0207_{-0.0004}^{+0.0004}$ & & & $375.83 / 491$ \\
\hline & $2 \mathrm{~T}$ & 5.95 & 0.320 & 0.0196 & \multirow[t]{2}{*}{47.67} & 0.0016 & $372.14 / 489$ \\
\hline & $\mathrm{T}+\mathrm{IC}$ & $6.53_{-0.38}^{+0.39}$ & $0.380_{-0.087}^{+0.064}$ & $0.0168_{-0.0023}^{+0.0033}$ & & $0.0012_{-0.0011}^{+0.0008}$ & $372.53 / 490$ \\
\hline
\end{tabular}


Table 5.3-Continued

\begin{tabular}{|c|c|c|c|c|c|c|c|}
\hline \multirow[b]{2}{*}{ Name } & \multirow[b]{2}{*}{ Model $^{\mathrm{a}}$} & \multicolumn{3}{|c|}{ Component 1} & \multicolumn{2}{|c|}{ Component 2} & \multirow[b]{2}{*}{$\chi^{2} /$ dof } \\
\hline & & $\begin{array}{c}k T \\
(\mathrm{keV})\end{array}$ & $\begin{array}{c}\text { abund } \\
\mathrm{Z}_{\odot}\end{array}$ & $\begin{array}{l}\text { Norm. }^{b} \\
\left(\mathrm{~cm}^{-5}\right)\end{array}$ & $\begin{array}{c}k T \\
(\mathrm{keV})\end{array}$ & Norm. $^{\mathrm{c}}$ & \\
\hline \multirow[t]{3}{*}{ A3395s } & $1 \mathrm{~T}$ & $5.75_{-0.66}^{+0.65}$ & $0.249_{-0.100}^{+0.102}$ & $0.0076_{-0.0003}^{+0.0004}$ & \multirow{3}{*}{8.64} & & $117.81 / 214$ \\
\hline & $2 \mathrm{~T}$ & 3.68 & 0.297 & 0.0040 & & 0.0039 & $115.72 / 212$ \\
\hline & $\mathrm{T}+\mathrm{IC}$ & $5.81_{-0.73}^{+0.75}$ & $0.279_{-0.112}^{+0.160}$ & $0.0067_{-0.0020}^{+0.0014}$ & & $<0.0009$ & $116.82 / 213$ \\
\hline \multirow[t]{3}{*}{ A0576 } & $1 \mathrm{~T}$ & $4.06_{-0.26}^{+0.28}$ & $0.376_{-0.083}^{+0.087}$ & $0.0244_{-0.0010}^{+0.0010}$ & \multirow{3}{*}{4.32} & & $175.59 / 226$ \\
\hline & $2 \mathrm{~T}$ & 3.83 & 0.377 & 0.0132 & & 0.0112 & $175.63 / 224$ \\
\hline & $\mathrm{T}+\mathrm{IC}$ & $3.90_{-0.33}^{+0.37}$ & $0.440_{-0.127}^{+0.127}$ & $0.0211_{-0.0031}^{+0.0041}$ & & $<0.0020$ & $174.37 / 225$ \\
\hline \multirow[t]{3}{*}{ A 0754} & $1 \mathrm{~T}$ & $9.35_{-0.33}^{+0.34}$ & $0.272_{-0.032}^{+0.032}$ & $0.0698_{-0.0007}^{+0.0007}$ & \multirow{3}{*}{15.52} & & $800.79 / 960$ \\
\hline & $2 \mathrm{~T}$ & 6.85 & 0.289 & 0.0423 & & 0.0295 & $778.89 / 958$ \\
\hline & $\mathrm{T}+\mathrm{IC}$ & $9.50_{-0.36}^{+0.38}$ & $0.306_{-0.040}^{+0.043}$ & $0.0649_{-0.0038}^{+0.0038}$ & & $0.0017_{-0.0013}^{+0.0013}$ & $788.88 / 959$ \\
\hline \multirow[t]{3}{*}{ HydraA } & $1 \mathrm{~T}$ & $3.98_{-0.09}^{+0.09}$ & $0.286_{-0.025}^{+0.026}$ & $0.0453_{-0.0007}^{+0.0007}$ & \multirow{4}{*}{6.51} & & $607.51 / 718$ \\
\hline & $2 \mathrm{~T}$ & 2.72 & 0.325 & 0.0290 & & 0.0181 & $590.83 / 716$ \\
\hline & $\mathrm{T}+\mathrm{IC}$ & $3.77_{-0.17}^{+0.15}$ & $0.338_{-0.042}^{+0.050}$ & $0.0391_{-0.0035}^{+0.0035}$ & & $0.0020_{-0.0011}^{+0.0011}$ & $598.26 / 717$ \\
\hline \multirow[t]{3}{*}{ A1060 } & $1 \mathrm{~T}$ & $3.20_{-0.05}^{+0.05}$ & $0.404_{-0.023}^{+0.023}$ & $0.0589_{-0.0007}^{+0.0007}$ & & & $858.66 / 972$ \\
\hline & $2 \mathrm{~T}$ & 3.28 & 0.404 & 0.0304 & \multirow[t]{2}{*}{3.12} & 0.0285 & $858.63 / 970$ \\
\hline & $\mathrm{T}+\mathrm{IC}$ & $3.07_{-0.08}^{+0.10}$ & $0.458_{-0.047}^{+0.040}$ & $0.0539_{-0.0027}^{+0.0037}$ & & $0.0017_{-0.0011}^{+0.0008}$ & $850.39 / 971$ \\
\hline \multirow[t]{3}{*}{ A 1367} & $1 \mathrm{~T}$ & $3.76_{-0.12}^{+0.12}$ & $0.302_{-0.037}^{+0.038}$ & $0.0324_{-0.0006}^{+0.0007}$ & \multirow{3}{*}{4.32} & & $481.82 / 603$ \\
\hline & $2 \mathrm{~T}$ & 1.27 & 0.307 & 0.0082 & & 0.0274 & $471.98 / 601$ \\
\hline & $\mathrm{T}+\mathrm{IC}$ & $3.40_{-0.18}^{+0.30}$ & $0.395_{-0.084}^{+0.081}$ & $0.0256_{-0.0028}^{+0.0041}$ & & $0.0022_{-0.0013}^{+0.0009}$ & $471.94 / 602$ \\
\hline \multirow[t]{3}{*}{ MKW4 } & $1 \mathrm{~T}$ & $1.68_{-0.11}^{+0.11}$ & $0.634_{-0.188}^{+0.239}$ & $0.0153_{-0.0021}^{+0.0025}$ & \multirow{4}{*}{1.60} & & $61.30 / 106$ \\
\hline & $2 \mathrm{~T}$ & 1.73 & 0.635 & 0.0104 & & 0.0049 & $61.30 / 104$ \\
\hline & $\mathrm{T}+\mathrm{IC}$ & $1.69_{-0.18}^{+0.11}$ & $0.634_{-0.186}^{+0.230}$ & $0.0153_{-0.0026}^{+0.0025}$ & & $<0.0004$ & $61.30 / 105$ \\
\hline \multirow[t]{3}{*}{ ZwCl1215 } & $1 \mathrm{~T}$ & $7.12_{-0.33}^{+0.34}$ & $0.284_{-0.037}^{+0.038}$ & $0.0255_{-0.0004}^{+0.0004}$ & & & $465.57 / 612$ \\
\hline & $2 \mathrm{~T}$ & 4.21 & 0.303 & 0.0058 & \multirow[t]{3}{*}{8.18} & 0.0201 & $464.25 / 610$ \\
\hline & $\mathrm{T}+\mathrm{IC}$ & $7.23_{-0.36}^{+0.37}$ & $0.307_{-0.053}^{+0.059}$ & $0.0237_{-0.0033}^{+0.0022}$ & & $<0.0016$ & $463.16 / 611$ \\
\hline \multirow[t]{3}{*}{ NGC4636 } & $1 \mathrm{~T}$ & $\begin{array}{l}0.92_{-0.09}^{+0.09} \\
\end{array}$ & $0.977_{-0.322}^{+0.630}$ & $0.0056_{-0.0016}^{+0.0017}$ & & & $229.06 / 363$ \\
\hline & $2 \mathrm{~T}$ & 26.36 & 1.988 & 0.0003 & \multirow[t]{2}{*}{0.75} & 0.0039 & $212.46 / 361$ \\
\hline & $\mathrm{T}+\mathrm{IC}$ & $0.75_{-0.06}^{+0.06}$ & $4.985_{-2.829}^{+-4.985}$ & $0.0016_{-0.0003}^{+0.0021}$ & & $0.0002_{-0.0001}^{+0.0001}$ & $213.53 / 362$ \\
\hline
\end{tabular}


Table 5.3-Continued

\begin{tabular}{|c|c|c|c|c|c|c|c|}
\hline \multirow[b]{2}{*}{ Name } & \multirow[b]{2}{*}{ Model $^{\mathrm{a}}$} & \multicolumn{3}{|c|}{ Component 1} & \multicolumn{2}{|c|}{ Component 2} & \multirow[b]{2}{*}{$\chi^{2} /$ dof } \\
\hline & & $\begin{array}{c}k T \\
(\mathrm{keV})\end{array}$ & $\begin{array}{c}\text { abund } \\
\mathrm{Z}_{\odot}\end{array}$ & $\begin{array}{l}\text { Norm. }^{\mathrm{b}} \\
\left(\mathrm{cm}^{-5}\right)\end{array}$ & $\begin{array}{c}k T \\
(\mathrm{keV})\end{array}$ & Norm. ${ }^{\mathrm{c}}$ & \\
\hline \multirow[t]{3}{*}{ A 3526} & $1 \mathrm{~T}$ & $3.95_{-0.18}^{+0.02}$ & $0.541_{-0.013}^{+0.009}$ & $0.1116_{-0.0029}^{+0.0019}$ & \multirow{3}{*}{4.02} & & $2020.47 / 1762$ \\
\hline & $2 \mathrm{~T}$ & 3.79 & 0.520 & 0.0549 & & 0.0544 & $2055.62 / 1760$ \\
\hline & $\mathrm{T}+\mathrm{IC}$ & $3.71_{-0.10}^{+0.05}$ & $0.577_{-0.012}^{+0.012}$ & $0.1049_{-0.0052}^{+0.0046}$ & & $0.0032_{-0.0012}^{+0.0009}$ & 2007.39/1761 \\
\hline \multirow[t]{3}{*}{ A1644 } & $1 \mathrm{~T}$ & $5.14_{-0.23}^{+0.24}$ & $0.294_{-0.045}^{+0.046}$ & $0.0442_{-0.0011}^{+0.0011}$ & \multirow{3}{*}{7.05} & & $394.74 / 534$ \\
\hline & $2 \mathrm{~T}$ & 4.02 & 0.312 & 0.0254 & & 0.0194 & $390.97 / 532$ \\
\hline & $\mathrm{T}+\mathrm{IC}$ & $5.09_{-0.26}^{+0.26}$ & $0.319_{-0.030}^{+0.054}$ & $0.0405_{-0.0047}^{+0.0044}$ & & $<0.0027$ & $393.22 / 533$ \\
\hline \multirow[t]{3}{*}{ A1650 } & $1 \mathrm{~T}$ & $5.94_{-0.16}^{+0.17}$ & $0.394_{-0.026}^{+0.026}$ & $0.0275_{-0.0003}^{+0.0003}$ & \multirow{4}{*}{7.57} & & $755.89 / 919$ \\
\hline & $2 \mathrm{~T}$ & 4.83 & 0.413 & 0.0151 & & 0.0126 & $752.04 / 917$ \\
\hline & $\mathrm{T}+\mathrm{IC}$ & $5.94_{-0.18}^{+0.19}$ & $0.451_{-0.057}^{+0.043}$ & $0.0238_{-0.0033}^{+0.0031}$ & & $0.0011_{-0.0010}^{+0.0010}$ & $749.68 / 918$ \\
\hline \multirow[t]{3}{*}{ A1651 } & $1 \mathrm{~T}$ & $6.45_{-0.35}^{+0.36}$ & $0.389_{-0.056}^{+0.057}$ & $0.0347_{-0.0009}^{+0.0009}$ & & & $212.15 / 335$ \\
\hline & $2 \mathrm{~T}$ & 3.41 & 0.406 & 0.0049 & \multirow[t]{3}{*}{7.02} & 0.0302 & $211.76 / 333$ \\
\hline & $\mathrm{T}+\mathrm{IC}$ & $6.45_{-0.43}^{+0.44}$ & $0.503_{-0.099}^{+0.120}$ & $0.0266_{-0.0042}^{+0.0044}$ & & $0.0025_{-0.0014}^{+0.0013}$ & $202.58 / 334$ \\
\hline \multirow[t]{3}{*}{ Coma } & $1 \mathrm{~T}$ & $8.51_{-0.11}^{+0.11}$ & $0.248_{-0.015}^{+0.015}$ & $0.2434_{-0.0013}^{+0.0013}$ & & & $1801.39 / 2167$ \\
\hline & $2 \mathrm{~T}$ & 7.35 & 0.248 & 0.0859 & \multirow[t]{2}{*}{9.29} & 0.1583 & $1797.98 / 2165$ \\
\hline & $\mathrm{T}+\mathrm{IC}$ & $8.51_{-0.11}^{+0.11}$ & $0.249_{-0.015}^{+0.015}$ & $0.2429_{-0.0038}^{+0.0017}$ & & $<0.0015$ & $1801.48 / 2166$ \\
\hline \multirow[t]{3}{*}{ NGC5044 } & $1 \mathrm{~T}$ & $1.20_{-0.04}^{+0.04}$ & $0.806_{-0.127}^{+0.0152}$ & $0.0245_{-0.0227}^{+0.0029}$ & \multirow{3}{*}{9.08} & & $391.41 / 497$ \\
\hline & $2 \mathrm{~T}$ & 1.10 & 0.862 & 0.0238 & & 0.0009 & $382.16 / 495$ \\
\hline & $\mathrm{T}+\mathrm{IC}$ & $1.10_{-0.08}^{+0.06}$ & $0.949_{-0.184}^{+0.255}$ & $0.0218_{-0.0035}^{+0.0035}$ & & $0.0005_{-0.0002}^{+0.0002}$ & $381.77 / 496$ \\
\hline \multirow[t]{3}{*}{ A3558 } & $1 \mathrm{~T}$ & $5.90_{-0.10}^{+0.10}$ & $0.324_{-0.015}^{+0.015}$ & $0.0663_{-0.0005}^{+0.0005}$ & \multirow{3}{*}{7.31} & & $1282.65 / 1465$ \\
\hline & $2 \mathrm{~T}$ & 5.26 & 0.330 & 0.0440 & & 0.0226 & $1282.28 / 1463$ \\
\hline & $\mathrm{T}+\mathrm{IC}$ & $5.90_{-0.10}^{+0.11}$ & $0.350_{-0.025}^{+0.016}$ & $0.0610_{-0.0040}^{+0.0040}$ & & $0.0017_{-0.0013}^{+0.0013}$ & $1277.76 / 1464$ \\
\hline \multirow[t]{3}{*}{ A3562 } & $1 \mathrm{~T}$ & $5.04_{-0.54}^{+0.61}$ & $0.414_{-0.144}^{+0.153}$ & $0.0175_{-0.0010}^{+0.0010}$ & \multirow{4}{*}{5.09} & & $58.83 / 134$ \\
\hline & $2 \mathrm{~T}$ & 5.00 & 0.414 & 0.0085 & & 0.0091 & $58.82 / 132$ \\
\hline & $\mathrm{T}+\mathrm{IC}$ & $5.12_{-0.56}^{+0.61}$ & $0.393_{-0.137}^{+0.159}$ & $0.0176_{-0.0039}^{+0.0011}$ & & $<0.0012$ & $59.51 / 133$ \\
\hline \multirow[t]{3}{*}{ A3571 } & $1 \mathrm{~T}$ & $7.20_{-0.14}^{+0.14}$ & $0.372_{-0.019}^{+0.019}$ & $0.1105_{-0.0008}^{+0.0008}$ & & & $1621.68 / 1883$ \\
\hline & $2 \mathrm{~T}$ & 7.24 & 0.372 & 0.0343 & \multirow[t]{2}{*}{7.18} & 0.0762 & $1621.67 / 1881$ \\
\hline & $\mathrm{T}+\mathrm{IC}$ & $7.20_{-0.15}^{+0.15}$ & $0.388_{-0.027}^{+0.024}$ & $0.1065_{-0.0038}^{+0.0044}$ & & $<0.0026$ & $1621.80 / 1882$ \\
\hline
\end{tabular}


Table 5.3-Continued

\begin{tabular}{|c|c|c|c|c|c|c|c|}
\hline \multirow[b]{2}{*}{ Name } & \multirow[b]{2}{*}{ Model $^{\mathrm{a}}$} & \multicolumn{3}{|c|}{ Component 1} & \multicolumn{2}{|c|}{ Component 2} & \multirow[b]{2}{*}{$\chi^{2} /$ dof } \\
\hline & & $\begin{array}{c}k T \\
(\mathrm{keV})\end{array}$ & $\begin{array}{c}\text { abund } \\
\mathrm{Z}_{\odot}\end{array}$ & $\begin{array}{l}\text { Norm. }^{b} \\
\left(\mathrm{~cm}^{-5}\right)\end{array}$ & $\begin{array}{c}k T \\
(\mathrm{keV})\end{array}$ & Norm. $^{\mathrm{c}}$ & \\
\hline \multirow[t]{3}{*}{ A1795 } & $1 \mathrm{~T}$ & $5.63_{-0.08}^{+0.08}$ & $0.365_{-0.013}^{+0.013}$ & $0.0794_{-0.0005}^{+0.0005}$ & \multirow{3}{*}{6.79} & & $1741.93 / 1916$ \\
\hline & $2 \mathrm{~T}$ & 4.33 & 0.380 & 0.0359 & & 0.0443 & $1731.99 / 1914$ \\
\hline & $\mathrm{T}+\mathrm{IC}$ & $5.63_{-0.09}^{+0.08}$ & $0.390_{-0.014}^{+0.014}$ & $0.0752_{-0.0031}^{+0.0031}$ & & $0.0014_{-0.0010}^{+0.0010}$ & $1673.45 / 1915$ \\
\hline \multirow[t]{3}{*}{ A3581 } & $1 \mathrm{~T}$ & $1.88_{-0.04}^{+0.04}$ & $0.557_{-0.055}^{+0.059}$ & $0.0272_{-0.0010}^{+0.0010}$ & \multirow{3}{*}{17.44} & & $428.74 / 555$ \\
\hline & $2 \mathrm{~T}$ & 1.73 & 0.568 & 0.0265 & & 0.0012 & $413.49 / 553$ \\
\hline & $\mathrm{T}+\mathrm{IC}$ & $1.74_{-0.07}^{+0.07}$ & $0.626_{-0.071}^{+0.079}$ & $0.0243_{-0.0016}^{+0.0016}$ & & $0.0008_{-0.0003}^{+0.0003}$ & $412.94 / 554$ \\
\hline \multirow[t]{3}{*}{ MKW8 } & $1 \mathrm{~T}$ & $3.35_{-0.21}^{+0.29}$ & $0.347_{-0.092}^{+0.099}$ & $0.0134_{-0.0007}^{+0.0007}$ & \multirow{3}{*}{5.23} & & $161.07 / 230$ \\
\hline & $2 \mathrm{~T}$ & 2.41 & 0.367 & 0.0083 & & 0.0054 & $158.11 / 228$ \\
\hline & $\mathrm{T}+\mathrm{IC}$ & $2.86_{-0.28}^{+0.63}$ & $0.567_{-0.257}^{+0.249}$ & $0.0107_{-0.0033}^{+0.0028}$ & & $<0.0017$ & $157.72 / 229$ \\
\hline \multirow[t]{3}{*}{ A 2029} & $1 \mathrm{~T}$ & $8.00_{-0.21}^{+0.21}$ & $0.428_{-0.029}^{+0.029}$ & $0.0781_{-0.0008}^{+0.0008}$ & \multirow{4}{*}{8.48} & & $877.33 / 952$ \\
\hline & $2 \mathrm{~T}$ & 0.29 & 0.458 & 0.5776 & & 0.0752 & $844.38 / 950$ \\
\hline & $\mathrm{T}+\mathrm{IC}$ & $8.13_{-0.23}^{+0.24}$ & $0.502_{-0.044}^{+0.034}$ & $0.0675_{-0.0044}^{+0.0043}$ & & $0.0034_{-0.0014}^{+0.0014}$ & $861.56 / 950$ \\
\hline \multirow[t]{3}{*}{ A 2052} & $1 \mathrm{~T}$ & $3.01_{-0.05}^{+0.05}$ & $0.504_{-0.029}^{+0.030}$ & $0.0479_{-0.0006}^{+0.0006}$ & & & $717.22 / 858$ \\
\hline & $2 \mathrm{~T}$ & 2.80 & 0.505 & 0.0250 & \multirow[t]{2}{*}{3.24} & 0.0229 & $716.88 / 856$ \\
\hline & $\mathrm{T}+\mathrm{IC}$ & $2.78_{-0.09}^{+0.12}$ & $0.623_{-0.071}^{+0.065}$ & $0.0410_{-0.0025}^{+0.0032}$ & & $0.0019_{-0.0009}^{+0.0007}$ & $706.30 / 857$ \\
\hline \multirow[t]{3}{*}{ MKW3S } & $1 \mathrm{~T}$ & $3.36_{-0.06}^{+0.06}$ & $0.385_{-0.026}^{+0.027}$ & $0.0396_{-0.0006}^{+0.0006}$ & \multirow{3}{*}{3.95} & & $722.14 / 847$ \\
\hline & $2 \mathrm{~T}$ & 2.89 & 0.389 & 0.0208 & & 0.0191 & $720.12 / 845$ \\
\hline & $\mathrm{T}+\mathrm{IC}$ & $3.21_{-0.12}^{+0.12}$ & $0.445_{-0.042}^{+0.042}$ & $0.0355_{-0.0027}^{+0.0028}$ & & $0.0013_{-0.0009}^{+0.0008}$ & $715.55 / 846$ \\
\hline \multirow[t]{3}{*}{ A 2065} & $1 \mathrm{~T}$ & $6.45_{-0.47}^{+0.53}$ & $0.263_{-0.076}^{+0.074}$ & $0.0291_{-0.0009}^{+0.0009}$ & \multirow{3}{*}{7.03} & & $166.28 / 258$ \\
\hline & $2 \mathrm{~T}$ & 4.81 & 0.266 & 0.0068 & & 0.0225 & $166.15 / 256$ \\
\hline & $\mathrm{T}+\mathrm{IC}$ & $6.45_{-0.49}^{+0.55}$ & $0.275_{-0.083}^{+0.087}$ & $0.0278_{-0.0037}^{+0.0021}$ & & $<0.0016$ & $165.89 / 257$ \\
\hline \multirow[t]{3}{*}{ A2063 } & $1 \mathrm{~T}$ & $\begin{array}{l}4.32_{-0.12}^{+0.15} \\
\end{array}$ & $0.345_{-0.033}^{+0.033}$ & $0.0371_{-0.0006}^{+0.0007}$ & \multirow{4}{*}{4.62} & & $649.81 / 783$ \\
\hline & $2 \mathrm{~T}$ & 4.07 & 0.345 & 0.0199 & & 0.0173 & $655.50 / 781$ \\
\hline & $\mathrm{T}+\mathrm{IC}$ & $4.22_{-0.15}^{+0.21}$ & $0.379_{-0.062}^{+0.044}$ & $0.0338_{-0.0031}^{+0.0038}$ & & $<0.0020$ & $654.33 / 782$ \\
\hline \multirow[t]{3}{*}{ A2142 } & $1 \mathrm{~T}$ & $10.39_{-0.75}^{+0.86}$ & $0.214_{-0.214}^{+0.333}$ & $0.0649_{-0.0045}^{+0.0044}$ & & & $47.14 / 98$ \\
\hline & $2 \mathrm{~T}$ & 8.96 & 0.241 & 0.0620 & \multirow[t]{2}{*}{64.00} & 0.0035 & $41.97 / 96$ \\
\hline & $\mathrm{T}+\mathrm{IC}$ & $9.59_{-1.04}^{+1.00}$ & $<0.631$ & $0.0572_{-0.0068}^{+0.0069}$ & & $0.0025_{-0.0019}^{+0.0019}$ & $42.51 / 97$ \\
\hline
\end{tabular}


Table 5.3-Continued

\begin{tabular}{|c|c|c|c|c|c|c|c|}
\hline \multirow[b]{2}{*}{ Name } & \multirow[b]{2}{*}{ Model $^{\mathrm{a}}$} & \multicolumn{3}{|c|}{ Component 1} & \multicolumn{2}{|c|}{ Component 2} & \multirow[b]{2}{*}{$\chi^{2} /$ dof } \\
\hline & & $\begin{array}{c}k T \\
(\mathrm{keV})\end{array}$ & $\begin{array}{c}\text { abund } \\
\mathrm{Z}_{\odot}\end{array}$ & $\begin{array}{l}\text { Norm. } \\
\left(\mathrm{cm}^{-5}\right)\end{array}$ & $\begin{array}{c}k T \\
(\mathrm{keV})\end{array}$ & Norm. $^{\mathrm{c}}$ & \\
\hline \multirow[t]{3}{*}{ A 2147} & $1 \mathrm{~T}$ & $4.99_{-0.53}^{+0.66}$ & $0.250_{-0.120}^{+0.126}$ & $0.0412_{-0.0023}^{+0.0023}$ & \multirow{3}{*}{6.84} & & $104.01 / 159$ \\
\hline & $2 \mathrm{~T}$ & 4.16 & 0.258 & 0.0258 & & 0.0157 & $103.35 / 157$ \\
\hline & $\mathrm{T}+\mathrm{IC}$ & $5.00_{-0.53}^{+0.67}$ & $0.246_{-0.116}^{+0.125}$ & $0.0414_{-0.0043}^{+0.0023}$ & & $<0.0013$ & $104.05 / 158$ \\
\hline \multirow[t]{3}{*}{ A2199 } & $1 \mathrm{~T}$ & $\begin{array}{l}4.45_{-0.09}^{+0.09} \\
\end{array}$ & $0.363_{-0.020}^{+0.021}$ & $0.1019_{-0.0011}^{+0.0011}$ & \multirow{3}{*}{5.48} & & $918.41 / 1078$ \\
\hline & $2 \mathrm{~T}$ & 3.59 & 0.379 & 0.0546 & & 0.0484 & $913.42 / 1076$ \\
\hline & $\mathrm{T}+\mathrm{IC}$ & $4.40_{-0.10}^{+0.10}$ & $0.376_{-0.024}^{+0.023}$ & $0.0983_{-0.0038}^{+0.0039}$ & & $<0.0024$ & $915.87 / 1077$ \\
\hline \multirow[t]{3}{*}{ A 2204} & $1 \mathrm{~T}$ & $7.10_{-0.23}^{+0.24}$ & $0.397_{-0.028}^{+0.029}$ & $0.0467_{-0.0006}^{+0.0006}$ & \multirow{4}{*}{12.93} & & $628.73 / 781$ \\
\hline & $2 \mathrm{~T}$ & 4.21 & 0.494 & 0.0255 & & 0.0232 & $608.41 / 779$ \\
\hline & $\mathrm{T}+\mathrm{IC}$ & $7.15_{-0.27}^{+0.27}$ & $0.487_{-0.063}^{+0.078}$ & $0.0380_{-0.0047}^{+0.0048}$ & & $0.0025_{-0.0014}^{+0.0013}$ & $619.76 / 780$ \\
\hline \multirow[t]{3}{*}{ A 2256} & $1 \mathrm{~T}$ & $7.11_{-0.38}^{+0.35}$ & $0.300_{-0.044}^{+0.045}$ & $0.0526_{-0.0009}^{+0.0009}$ & & & $342.00 / 443$ \\
\hline & $2 \mathrm{~T}$ & 0.44 & 0.330 & 0.0966 & \multirow[t]{3}{*}{7.76} & 0.0500 & $324.40 / 441$ \\
\hline & $\mathrm{T}+\mathrm{IC}$ & $7.06_{-0.38}^{+0.38}$ & $0.328_{-0.048}^{+0.057}$ & $0.0477_{-0.0034}^{+0.0034}$ & & $0.0017_{-0.0011}^{+0.0011}$ & $337.48 / 442$ \\
\hline \multirow[t]{3}{*}{ A 2255} & $1 \mathrm{~T}$ & $7.44_{-0.71}^{+0.80}$ & $0.269_{-0.104}^{+0.106}$ & $0.0237_{-0.0008}^{+0.0008}$ & & & $104.16 / 193$ \\
\hline & $2 \mathrm{~T}$ & 7.21 & 0.263 & 0.0130 & \multirow[t]{2}{*}{7.85} & 0.0108 & $104.14 / 191$ \\
\hline & $\mathrm{T}+\mathrm{IC}$ & $7.45_{-0.73}^{+0.79}$ & $0.263_{-0.098}^{+0.113}$ & $0.0237_{-0.0017}^{+0.0008}$ & & $<0.0005$ & $104.16 / 192$ \\
\hline \multirow[t]{3}{*}{ A 3667} & $1 \mathrm{~T}$ & $6.61_{-0.11}^{+0.11}$ & $0.266_{-0.015}^{+0.015}$ & $0.0758_{-0.0005}^{+0.0005}$ & \multirow{3}{*}{9.95} & & $1498.83 / 1652$ \\
\hline & $2 \mathrm{~T}$ & 4.88 & 0.292 & 0.0427 & & 0.0343 & $1482.99 / 1650$ \\
\hline & $\mathrm{T}+\mathrm{IC}$ & $6.76_{-0.13}^{+0.18}$ & $0.307_{-0.024}^{+0.027}$ & $0.0642_{-0.0042}^{+0.0042}$ & & $0.0037_{-0.0013}^{+0.0013}$ & $1487.65 / 1651$ \\
\hline \multirow[t]{3}{*}{ S1101 } & $1 \mathrm{~T}$ & $2.65_{-0.06}^{+0.06}$ & $0.336_{-0.037}^{+0.038}$ & $0.0259_{-0.0006}^{+0.0006}$ & \multirow{4}{*}{3.68} & & $418.31 / 534$ \\
\hline & $2 \mathrm{~T}$ & 2.09 & 0.332 & 0.0172 & & 0.0097 & $414.16 / 532$ \\
\hline & $\mathrm{T}+\mathrm{IC}$ & $2.58_{-0.07}^{+0.12}$ & $0.352_{-0.046}^{+0.050}$ & $0.0251_{-0.0015}^{+0.0012}$ & & $<0.0007$ & $417.42 / 533$ \\
\hline \multirow[t]{3}{*}{ A 2589} & $1 \mathrm{~T}$ & $3.68_{-0.12}^{+0.13}$ & $0.542_{-0.050}^{+0.052}$ & $0.0205_{-0.0004}^{+0.0004}$ & & & $334.55 / 446$ \\
\hline & $2 \mathrm{~T}$ & 0.01 & 0.542 & 0.0013 & \multirow[t]{3}{*}{3.68} & 0.0205 & $334.55 / 444$ \\
\hline & $\mathrm{T}+\mathrm{IC}$ & $3.27_{-0.12}^{+0.14}$ & $0.826_{-0.087}^{+0.074}$ & $0.0155_{-0.0026}^{+0.0024}$ & & $0.0015_{-0.0004}^{+0.0007}$ & $325.18 / 444$ \\
\hline \multirow[t]{3}{*}{ A 2597} & $1 \mathrm{~T}$ & $3.35_{-0.07}^{+0.07}$ & $0.329_{-0.024}^{+0.024}$ & $0.0274_{-0.0004}^{+0.0004}$ & & & $626.62 / 721$ \\
\hline & $2 \mathrm{~T}$ & 2.25 & 0.357 & 0.0176 & \multirow[t]{2}{*}{5.44} & 0.0112 & $601.45 / 719$ \\
\hline & $\mathrm{T}+\mathrm{IC}$ & $2.99_{-0.16}^{+0.15}$ & $0.455_{-0.062}^{+0.080}$ & $0.0216_{-0.0022}^{+0.0021}$ & & $0.0017_{-0.0006}^{+0.0006}$ & $605.99 / 720$ \\
\hline
\end{tabular}


Table 5.3-Continued

\begin{tabular}{|c|c|c|c|c|c|c|c|}
\hline \multirow[b]{2}{*}{ Name } & \multirow[b]{2}{*}{ Model $^{\mathrm{a}}$} & \multicolumn{3}{|c|}{ Component 1} & \multicolumn{2}{|c|}{ Component 2} & \multirow[b]{2}{*}{$\chi^{2} /$ dof } \\
\hline & & $\begin{array}{c}k T \\
(\mathrm{keV})\end{array}$ & $\begin{array}{c}\text { abund } \\
\mathrm{Z}_{\odot}\end{array}$ & $\begin{array}{l}\text { Norm. }^{\mathrm{b}} \\
\left(\mathrm{cm}^{-5}\right)\end{array}$ & $\begin{array}{c}k T \\
(\mathrm{keV})\end{array}$ & Norm. $^{\mathrm{c}}$ & \\
\hline \multirow[t]{3}{*}{ A 2634} & $1 \mathrm{~T}$ & $4.50_{-0.45}^{+0.56}$ & $0.292_{-0.140}^{+0.148}$ & $0.0182_{-0.0010}^{+0.0011}$ & \multirow{3}{*}{4.36} & & 108.16/140 \\
\hline & $2 \mathrm{~T}$ & 4.63 & 0.291 & 0.0097 & & 0.0086 & $108.18 / 138$ \\
\hline & $\mathrm{T}+\mathrm{IC}$ & $4.57_{-0.49}^{+0.57}$ & $0.267_{-0.126}^{+0.130}$ & $0.0186_{-0.0032}^{+0.0006}$ & & $<0.0010$ & $107.64 / 139$ \\
\hline \multirow[t]{3}{*}{ A 2657} & $1 \mathrm{~T}$ & $5.14_{-0.28}^{+0.30}$ & $0.284_{-0.063}^{+0.065}$ & $0.0256_{-0.0007}^{+0.0008}$ & \multirow{4}{*}{7.80} & & $273.87 / 356$ \\
\hline & $2 \mathrm{~T}$ & 2.15 & 0.381 & 0.0111 & & 0.0166 & $263.96 / 354$ \\
\hline & $\mathrm{T}+\mathrm{IC}$ & $5.15_{-0.29}^{+0.32}$ & $0.281_{-0.061}^{+0.068}$ & $0.0257_{-0.0033}^{+0.0007}$ & & $<0.0010$ & $274.20 / 355$ \\
\hline \multirow[t]{3}{*}{ A 4038} & $1 \mathrm{~T}$ & $3.17_{-0.05}^{+0.05}$ & $0.371_{-0.024}^{+0.025}$ & $0.0593_{-0.0008}^{+0.0008}$ & & & $870.12 / 1058$ \\
\hline & $2 \mathrm{~T}$ & 2.53 & 0.384 & 0.0414 & \multirow[t]{3}{*}{4.80} & 0.0193 & $858.72 / 1056$ \\
\hline & $\mathrm{T}+\mathrm{IC}$ & $3.03_{-0.10}^{+0.11}$ & $0.424_{-0.043}^{+0.045}$ & $0.0538_{-0.0030}^{+0.0033}$ & & $0.0017_{-0.0010}^{+0.0009}$ & $862.28 / 1057$ \\
\hline \multirow[t]{3}{*}{ A 4059} & $1 \mathrm{~T}$ & $4.22_{-0.12}^{+0.13}$ & $0.428_{-0.035}^{+0.043}$ & $0.0340_{-0.0006}^{+0.0006}$ & & & $481.92 / 694$ \\
\hline & $2 \mathrm{~T}$ & 2.57 & 0.442 & 0.0066 & \multirow[t]{2}{*}{4.68} & 0.0280 & $478.65 / 692$ \\
\hline & $\mathrm{T}+\mathrm{IC}$ & $4.21_{-0.14}^{+0.12}$ & $0.432_{-0.035}^{+0.053}$ & $0.0340_{-0.0030}^{+0.0006}$ & & $<0.0009$ & $480.55 / 693$ \\
\hline
\end{tabular}

a Parameters for the $2 \mathrm{~T}$ model are unconstrained.

${ }^{\mathrm{b}}$ Normalization of the APEC thermal spectrum, which is given by $\left\{10^{-14} /[4 \pi(1+\right.$ $\left.\left.z)^{2} D_{A}^{2}\right]\right\} \int n_{e} n_{H} d V$, where $z$ is the redshift, $D_{A}$ is the angular diameter distance, $n_{e}$ is the electron density, $n_{H}$ is the ionized hydrogen density, and $V$ is the volume of the cluster.

${ }^{c}$ Value is the normalization of the power-law component for the $\mathrm{T}+\mathrm{IC}$ model, which is the photon flux at a photon energy of $1 \mathrm{keV}$ in units of photons $\mathrm{cm}^{-2} \mathrm{~s}^{-1} \mathrm{keV}^{-1}$. For the $2 \mathrm{~T}$ model, the value is the normalization of the second APEC thermal model in units of $\mathrm{cm}^{-5}$. 
Table 5.4. Joint Thermal and Nonthermal Fits to the EPIC and BAT Spectra $(3-195 \mathrm{keV})$

\begin{tabular}{|c|c|c|c|c|c|c|c|}
\hline \multirow[b]{2}{*}{ Name } & \multirow[b]{2}{*}{ Model $^{\mathrm{a}}$} & \multicolumn{3}{|c|}{ Component 1} & \multicolumn{2}{|c|}{ Component 2} & \multirow[b]{2}{*}{$\chi^{2} /$ dof } \\
\hline & & $\begin{array}{c}k T \\
(\mathrm{keV})\end{array}$ & $\begin{array}{c}\text { abund } \\
\mathrm{Z}_{\odot}\end{array}$ & $\begin{array}{l}\text { Norm. } \\
\left(\mathrm{cm}^{-5}\right)\end{array}$ & $\begin{array}{c}k T \\
(\mathrm{keV})\end{array}$ & Norm. $^{\mathrm{c}}$ & \\
\hline \multirow[t]{3}{*}{ A0085 } & $1 \mathrm{~T}$ & $6.93_{-0.26}^{+0.31}$ & $0.363_{-0.033}^{+0.034}$ & $0.0746_{-0.0015}^{+0.0016}$ & \multirow{3}{*}{6.92} & & $410.01 / 534$ \\
\hline & $2 \mathrm{~T}$ & 6.95 & 0.362 & 0.0448 & & 0.0297 & $409.98 / 532$ \\
\hline & $\mathrm{T}+\mathrm{IC}$ & $6.91_{-0.27}^{+0.30}$ & $0.363_{-0.031}^{+0.037}$ & $0.0746_{-0.0022}^{+0.0017}$ & & $<0.0007$ & $411.45 / 533$ \\
\hline \multirow[t]{3}{*}{ A0119 } & $1 \mathrm{~T}$ & $6.72_{-0.79}^{+0.88}$ & $0.243_{-0.079}^{+0.082}$ & $0.0285_{-0.0015}^{+0.0020}$ & \multirow{3}{*}{6.74} & & $115.57 / 162$ \\
\hline & $2 \mathrm{~T}$ & 6.62 & 0.232 & 0.0168 & & 0.0115 & $117.76 / 160$ \\
\hline & $\mathrm{T}+\mathrm{IC}$ & $6.76_{-0.85}^{+0.83}$ & $0.238_{-0.075}^{+0.086}$ & $0.0285_{-0.0027}^{+0.0021}$ & & $<0.0009$ & $115.70 / 161$ \\
\hline \multirow[t]{3}{*}{ A0133 } & $1 \mathrm{~T}$ & $4.27_{-0.27}^{+0.34}$ & $0.442_{-0.051}^{+0.055}$ & $0.0211_{-0.0011}^{+0.0012}$ & \multirow{3}{*}{4.24} & & $134.52 / 237$ \\
\hline & $2 \mathrm{~T}$ & 63.88 & 0.443 & 0.0001 & & 0.0211 & $134.39 / 235$ \\
\hline & $\mathrm{T}+\mathrm{IC}$ & $13.47_{-1.55}^{+2.18}$ & $<0.840$ & $0.0645_{-0.0126}^{+0.0155}$ & & $<0.0018$ & $383.72 / 236$ \\
\hline \multirow[t]{3}{*}{ NGC507 } & $1 \mathrm{~T}$ & $1.85_{-0.27}^{+0.35}$ & $0.814_{-0.422}^{+0.736}$ & $0.0073_{-0.0019}^{+0.0029}$ & \multirow{3}{*}{5.69} & & $64.53 / 99$ \\
\hline & $2 \mathrm{~T}$ & 1.35 & 0.841 & 0.0084 & & 0.0009 & $64.60 / 97$ \\
\hline & $\mathrm{T}+\mathrm{IC}$ & $1.62_{-0.40}^{+0.43}$ & $1.457_{-0.951}^{+-1.457}$ & $0.0051_{-0.0035}^{+0.0044}$ & & $<0.0006$ & $62.90 / 98$ \\
\hline \multirow[t]{3}{*}{ A0262 } & $1 \mathrm{~T}$ & $2.33_{-0.08}^{+0.09}$ & $0.412_{-0.055}^{+0.059}$ & $0.0531_{-0.0026}^{+0.0027}$ & \multirow{4}{*}{2.45} & & $297.16 / 379$ \\
\hline & $2 \mathrm{~T}$ & 2.32 & 0.412 & 0.0479 & & 0.0052 & $297.15 / 377$ \\
\hline & $\mathrm{T}+\mathrm{IC}$ & $2.33_{-0.09}^{+0.09}$ & $0.404_{-0.049}^{+0.065}$ & $0.0533_{-0.0027}^{+0.0026}$ & & $<0.0004$ & $297.46 / 378$ \\
\hline \multirow[t]{3}{*}{ A0400 } & $1 \mathrm{~T}$ & $2.34_{-0.23}^{+0.30}$ & $0.352_{-0.143}^{+0.168}$ & $0.0195_{-0.0027}^{+0.0031}$ & & & $138.40 / 159$ \\
\hline & $2 \mathrm{~T}$ & 2.28 & 0.352 & 0.0104 & \multirow[t]{3}{*}{2.41} & 0.0091 & $138.40 / 157$ \\
\hline & $\mathrm{T}+\mathrm{IC}$ & $2.29_{-0.20}^{+0.36}$ & $0.348_{-0.138}^{+0.177}$ & $0.0200_{-0.0033}^{+0.0027}$ & & $<0.0002$ & $138.40 / 158$ \\
\hline \multirow[t]{3}{*}{ A0399 } & $1 \mathrm{~T}$ & $7.63_{-0.67}^{+0.71}$ & $0.233_{-0.056}^{+0.058}$ & $0.0349_{-0.0014}^{+0.0014}$ & & & $152.92 / 229$ \\
\hline & $2 \mathrm{~T}$ & 7.69 & 0.233 & 0.0105 & \multirow[t]{2}{*}{7.61} & 0.0244 & $152.92 / 227$ \\
\hline & $\mathrm{T}+\mathrm{IC}$ & $7.73_{-0.77}^{+0.62}$ & $0.235_{-0.058}^{+0.057}$ & $0.0347_{-0.0019}^{+0.0016}$ & & $<0.0007$ & $152.93 / 228$ \\
\hline \multirow[t]{3}{*}{ A3112 } & $1 \mathrm{~T}$ & $5.29_{-0.22}^{+0.27}$ & $0.455_{-0.031}^{+0.032}$ & $0.0339_{-0.0011}^{+0.0011}$ & \multirow{3}{*}{6.62} & & $374.66 / 448$ \\
\hline & $2 \mathrm{~T}$ & 3.44 & 0.497 & 0.0145 & & 0.0206 & $370.33 / 446$ \\
\hline & $\mathrm{T}+\mathrm{IC}$ & $5.14_{-0.24}^{+0.29}$ & $0.525_{-0.066}^{+0.065}$ & $0.0297_{-0.0029}^{+0.0035}$ & & $0.0014_{-0.0011}^{+0.0009}$ & $366.25 / 447$ \\
\hline \multirow[t]{3}{*}{ Fornax } & $1 \mathrm{~T}$ & $2.01_{-0.13}^{+0.17}$ & $0.253_{-0.085}^{+0.096}$ & $0.0191_{-0.0021}^{+0.0022}$ & \multirow{3}{*}{3.38} & & $471.94 / 503$ \\
\hline & $2 \mathrm{~T}$ & 1.59 & 0.254 & 0.0177 & & 0.0039 & $470.56 / 501$ \\
\hline & $\mathrm{T}+\mathrm{IC}$ & $1.54_{-0.26}^{+0.27}$ & $0.519_{-0.194}^{+0.543}$ & $0.0175_{-0.0045}^{+0.0035}$ & & $0.0009_{-0.0005}^{+0.0004}$ & $464.80 / 502$ \\
\hline
\end{tabular}


Table 5.4-Continued

\begin{tabular}{|c|c|c|c|c|c|c|c|}
\hline \multirow[b]{2}{*}{ Name } & \multirow[b]{2}{*}{ Model $^{\mathrm{a}}$} & \multicolumn{3}{|c|}{ Component 1} & \multicolumn{2}{|c|}{ Component 2} & \multirow[b]{2}{*}{$\chi^{2} /$ dof } \\
\hline & & $\begin{array}{c}k T \\
(\mathrm{keV})\end{array}$ & $\begin{array}{c}\text { abund } \\
\mathrm{Z}_{\odot}\end{array}$ & $\begin{array}{l}\text { Norm. }^{b} \\
\left(\mathrm{~cm}^{-5}\right)\end{array}$ & $\begin{array}{c}k T \\
(\mathrm{keV})\end{array}$ & Norm. $^{\mathrm{c}}$ & \\
\hline \multirow[t]{3}{*}{$2 \mathrm{~A} 0335$} & $1 \mathrm{~T}$ & $3.22_{-0.12}^{+0.13}$ & $0.400_{-0.035}^{+0.036}$ & $0.0941_{-0.0037}^{+0.0037}$ & \multirow{3}{*}{3.93} & & $254.73 / 381$ \\
\hline & $2 \mathrm{~T}$ & 2.67 & 0.411 & 0.0553 & & 0.0407 & $254.16 / 379$ \\
\hline & $\mathrm{T}+\mathrm{IC}$ & $3.22_{-0.13}^{+0.13}$ & $0.399_{-0.032}^{+0.040}$ & $0.0943_{-0.0042}^{+0.0036}$ & & $<0.0010$ & $254.74 / 380$ \\
\hline \multirow[t]{3}{*}{ IIIZw54 } & $1 \mathrm{~T}$ & $3.04_{-0.23}^{+0.28}$ & $0.242_{-0.063}^{+0.068}$ & $0.0170_{-0.0015}^{+0.0015}$ & \multirow{3}{*}{3.07} & & $156.99 / 228$ \\
\hline & $2 \mathrm{~T}$ & 3.01 & 0.242 & 0.0089 & & 0.0081 & $156.99 / 226$ \\
\hline & $\mathrm{T}+\mathrm{IC}$ & $2.80_{-0.42}^{+0.50}$ & $0.276_{-0.099}^{+0.129}$ & $0.0158_{-0.0024}^{+0.0025}$ & & $<0.0012$ & $157.99 / 227$ \\
\hline \multirow[t]{3}{*}{ A3158 } & $1 \mathrm{~T}$ & $6.32_{-0.51}^{+0.55}$ & $0.348_{-0.061}^{+0.063}$ & $0.0388_{-0.0019}^{+0.0019}$ & \multirow{3}{*}{6.31} & & $154.53 / 217$ \\
\hline & $2 \mathrm{~T}$ & 0.02 & 0.350 & 0.0001 & & 0.0388 & $154.78 / 215$ \\
\hline & $\mathrm{T}+\mathrm{IC}$ & $5.88_{-0.35}^{+0.35}$ & $0.343_{-0.061}^{+0.065}$ & $0.0397_{-0.0034}^{+0.0019}$ & & $<0.0014$ & $268.81 / 359$ \\
\hline \multirow[t]{3}{*}{ NGC1550 } & $1 \mathrm{~T}$ & $1.54_{-0.13}^{+0.19}$ & $0.297_{-0.168}^{+0.222}$ & $0.0287_{-0.0061}^{+0.0075}$ & \multirow{3}{*}{1.67} & & $82.84 / 128$ \\
\hline & $2 \mathrm{~T}$ & 1.45 & 0.297 & 0.0168 & & 0.0119 & $82.83 / 126$ \\
\hline & $\mathrm{T}+\mathrm{IC}$ & $1.39_{-0.30}^{+0.31}$ & $0.380_{-0.221}^{+0.385}$ & $0.0290_{-0.0080}^{+0.0150}$ & & $<0.0009$ & $81.43 / 127$ \\
\hline \multirow[t]{3}{*}{ EXO0422 } & $1 \mathrm{~T}$ & $3.21_{-0.13}^{+0.15}$ & $0.336_{-0.034}^{+0.035}$ & $0.0289_{-0.0013}^{+0.0013}$ & \multirow{3}{*}{3.58} & & $330.69 / 446$ \\
\hline & $2 \mathrm{~T}$ & 2.73 & 0.339 & 0.0129 & & 0.0162 & $330.65 / 444$ \\
\hline & $\mathrm{T}+\mathrm{IC}$ & $2.86_{-0.23}^{+0.40}$ & $0.424_{-0.107}^{+0.097}$ & $0.0262_{-0.0026}^{+0.0035}$ & & $<0.0019$ & $330.45 / 445$ \\
\hline \multirow[t]{3}{*}{ A 3266} & $1 \mathrm{~T}$ & $8.82_{-0.50}^{+0.45}$ & $0.198_{-0.033}^{+0.033}$ & $0.0708_{-0.0012}^{+0.0016}$ & \multirow{3}{*}{8.97} & & $563.68 / 730$ \\
\hline & $2 \mathrm{~T}$ & 4.35 & 0.199 & 0.0025 & & 0.0687 & $563.36 / 728$ \\
\hline & $\mathrm{T}+\mathrm{IC}$ & $8.55_{-0.38}^{+0.57}$ & $0.208_{-0.034}^{+0.036}$ & $0.0678_{-0.0036}^{+0.0036}$ & & $<0.0027$ & $561.45 / 729$ \\
\hline \multirow[t]{3}{*}{ A0496 } & $1 \mathrm{~T}$ & $4.59_{-0.13}^{+0.14}$ & $0.388_{-0.022}^{+0.022}$ & $0.0805_{-0.0016}^{+0.00016}$ & \multirow{3}{*}{5.03} & & $631.47 / 766$ \\
\hline & $2 \mathrm{~T}$ & 4.35 & 0.388 & 0.0519 & & 0.0287 & $631.48 / 764$ \\
\hline & $\mathrm{T}+\mathrm{IC}$ & $4.59_{-0.12}^{+0.16}$ & $0.385_{-0.021}^{+0.023}$ & $0.0806_{-0.0023}^{+0.0015}$ & & $<0.0006$ & $630.87 / 765$ \\
\hline \multirow[t]{3}{*}{ A3376 } & $1 \mathrm{~T}$ & $5.78_{-0.96}^{+1.13}$ & $0.445_{-0.128}^{+0.145}$ & $0.0086_{-0.0008}^{+0.0010}$ & \multirow{3}{*}{6.85} & & $64.00 / 84$ \\
\hline & $2 \mathrm{~T}$ & 1.86 & 0.531 & 0.0028 & & 0.0070 & $63.27 / 82$ \\
\hline & $\mathrm{T}+\mathrm{IC}$ & $5.28_{-1.33}^{+1.31}$ & $0.611_{-0.241}^{+0.672}$ & $0.0068_{-0.0034}^{+0.0028}$ & & $<0.0018$ & $63.25 / 83$ \\
\hline \multirow[t]{3}{*}{ A3391 } & $1 \mathrm{~T}$ & $6.84_{-0.48}^{+0.58}$ & $0.313_{-0.052}^{+0.054}$ & $0.0200_{-0.0007}^{+0.0008}$ & \multirow{3}{*}{55.44} & & $203.38 / 303$ \\
\hline & $2 \mathrm{~T}$ & 6.39 & 0.321 & 0.0191 & & 0.0013 & $200.90 / 301$ \\
\hline & $\mathrm{T}+\mathrm{IC}$ & $6.72_{-0.59}^{+0.73}$ & $0.376_{-0.107}^{+0.065}$ & $0.0197_{-0.0048}^{+0.0011}$ & & $<0.0019$ & $202.47 / 302$ \\
\hline
\end{tabular}


Table 5.4-Continued

\begin{tabular}{|c|c|c|c|c|c|c|c|}
\hline \multirow[b]{2}{*}{ Name } & \multirow[b]{2}{*}{ Model $^{\mathrm{a}}$} & \multicolumn{3}{|c|}{ Component 1} & \multicolumn{2}{|c|}{ Component 2} & \multirow[b]{2}{*}{$\chi^{2} /$ dof } \\
\hline & & $\begin{array}{c}k T \\
(\mathrm{keV})\end{array}$ & $\begin{array}{c}\text { abund } \\
\mathrm{Z}_{\odot}\end{array}$ & $\begin{array}{l}\text { Norm. }^{\mathrm{b}} \\
\left(\mathrm{cm}^{-5}\right)\end{array}$ & $\begin{array}{c}k T \\
(\mathrm{keV})\end{array}$ & Norm. $^{\mathrm{c}}$ & \\
\hline \multirow[t]{3}{*}{ A3395s } & $1 \mathrm{~T}$ & $5.93_{-1.11}^{+1.22}$ & $0.245_{-0.103}^{+0.111}$ & $0.0075_{-0.0007}^{+0.0010}$ & \multirow{3}{*}{7.80} & & $57.04 / 124$ \\
\hline & $2 \mathrm{~T}$ & 4.31 & 0.261 & 0.0040 & & 0.0037 & $56.21 / 122$ \\
\hline & $\mathrm{T}+\mathrm{IC}$ & $6.02_{-1.19}^{+1.30}$ & $0.239_{-0.101}^{+0.169}$ & $0.0075_{-0.0027}^{+0.0010}$ & & $<0.0010$ & $56.97 / 123$ \\
\hline \multirow[t]{3}{*}{ A0576 } & $1 \mathrm{~T}$ & $4.39_{-0.54}^{+0.61}$ & $0.364_{-0.084}^{+0.081}$ & $0.0231_{-0.0019}^{+0.0026}$ & \multirow{3}{*}{6.30} & & $91.72 / 129$ \\
\hline & $2 \mathrm{~T}$ & 2.73 & 0.429 & 0.0136 & & 0.0112 & $91.07 / 127$ \\
\hline & $\mathrm{T}+\mathrm{IC}$ & $4.11_{-0.57}^{+0.76}$ & $0.406_{-0.114}^{+0.129}$ & $0.0215_{-0.0038}^{+0.0038}$ & & $<0.0019$ & $91.09 / 128$ \\
\hline \multirow[t]{3}{*}{ A0754 } & $1 \mathrm{~T}$ & $9.71_{-0.44}^{+0.45}$ & $0.284_{-0.034}^{+0.035}$ & $0.0690_{-0.0011}^{+0.0011}$ & \multirow{4}{*}{16.64} & & $533.95 / 645$ \\
\hline & $2 \mathrm{~T}$ & 7.56 & 0.294 & 0.0480 & & 0.0229 & $526.36 / 643$ \\
\hline & $\mathrm{T}+\mathrm{IC}$ & $9.69_{-0.45}^{+0.46}$ & $0.292_{-0.039}^{+0.040}$ & $0.0674_{-0.0045}^{+0.0027}$ & & $<0.0024$ & $530.31 / 644$ \\
\hline \multirow[t]{3}{*}{ HydraA } & $1 \mathrm{~T}$ & $4.39_{-0.18}^{+0.19}$ & $0.282_{-0.026}^{+0.026}$ & $0.0414_{-0.0013}^{+0.0014}$ & & & $330.74 / 443$ \\
\hline & $2 \mathrm{~T}$ & 3.89 & 0.297 & 0.0342 & \multirow[t]{2}{*}{6.83} & 0.0080 & $330.59 / 441$ \\
\hline & $\mathrm{T}+\mathrm{IC}$ & $4.32_{-0.24}^{+0.25}$ & $0.292_{-0.035}^{+0.038}$ & $0.0399_{-0.0031}^{+0.0028}$ & & $<0.0016$ & $330.55 / 442$ \\
\hline \multirow[t]{3}{*}{ A1060 } & $1 \mathrm{~T}$ & $3.43_{-0.09}^{+0.09}$ & $0.383_{-0.023}^{+0.025}$ & $0.0557_{-0.0012}^{+0.0016}$ & \multirow{3}{*}{3.42} & & $519.24 / 641$ \\
\hline & $2 \mathrm{~T}$ & 3.42 & 0.383 & 0.0289 & & 0.0268 & $519.14 / 639$ \\
\hline & $\mathrm{T}+\mathrm{IC}$ & $3.42_{-0.10}^{+0.09}$ & $0.382_{-0.022}^{+0.028}$ & $0.0558_{-0.0020}^{+0.0015}$ & & $<0.0006$ & $519.22 / 640$ \\
\hline \multirow[t]{3}{*}{ A1367 } & $1 \mathrm{~T}$ & $4.17_{-0.23}^{+0.26}$ & $0.298_{-0.039}^{+0.040}$ & $0.0297_{-0.0014}^{+0.0015}$ & \multirow{3}{*}{4.33} & & $259.14 / 344$ \\
\hline & $2 \mathrm{~T}$ & 1.65 & 0.302 & 0.0031 & & 0.0278 & $258.76 / 342$ \\
\hline & $\mathrm{T}+\mathrm{IC}$ & $3.92_{-0.33}^{+0.33}$ & $0.349_{-0.058}^{+0.063}$ & $0.0262_{-0.0032}^{+0.0032}$ & & $0.0013_{-0.0011}^{+0.0011}$ & $254.96 / 343$ \\
\hline \multirow[t]{3}{*}{ MKW4 } & $1 \mathrm{~T}$ & $\begin{array}{l}1.73_{-0.26}^{+0.28} \\
\end{array}$ & $0.888_{-0.516}^{+1.050}$ & $0.0124_{-0.0040}^{+0.0065}$ & \multirow{3}{*}{1.80} & & $27.32 / 48$ \\
\hline & $2 \mathrm{~T}$ & 1.72 & 0.887 & 0.0090 & & 0.0035 & $27.54 / 46$ \\
\hline & $\mathrm{T}+\mathrm{IC}$ & $1.65_{-0.43}^{+0.35}$ & $0.950_{-0.582}^{+3.037}$ & $0.0120_{-0.0080}^{+0.0070}$ & & $<0.0007$ & $0.00 / 27$ \\
\hline \multirow[t]{3}{*}{ ZwCl1215 } & $1 \mathrm{~T}$ & $7.64_{-0.50}^{+0.51}$ & $0.299_{-0.042}^{+0.043}$ & $0.0247_{-0.0007}^{+0.0008}$ & \multirow{4}{*}{7.58} & & $279.58 / 372$ \\
\hline & $2 \mathrm{~T}$ & 7.92 & 0.299 & 0.0044 & & 0.0203 & $279.58 / 370$ \\
\hline & $\mathrm{T}+\mathrm{IC}$ & $7.69_{-0.54}^{+0.47}$ & $0.302_{-0.042}^{+0.047}$ & $0.0247_{-0.0022}^{+0.0008}$ & & $<0.0008$ & $278.18 / 371$ \\
\hline \multirow[t]{3}{*}{ NGC4636 } & $1 \mathrm{~T}$ & $2.30_{-1.02}^{+3.13}$ & $0.197_{-0.197}^{+4.127}$ & $0.0019_{-0.0019}^{+0.0033}$ & & & $101.60 / 154$ \\
\hline & $2 \mathrm{~T}$ & 17.23 & 1.654 & 0.0004 & \multirow[t]{2}{*}{0.72} & 0.0036 & $99.60 / 152$ \\
\hline & $\mathrm{T}+\mathrm{IC}$ & $0.37_{-0.31}^{+2.82}$ & $<0.000$ & $0.0252_{-0.0243}^{+173.0428}$ & & $<0.0003$ & $99.45 / 153$ \\
\hline
\end{tabular}


Table 5.4-Continued

\begin{tabular}{|c|c|c|c|c|c|c|c|}
\hline \multirow[b]{2}{*}{ Name } & \multirow[b]{2}{*}{ Model $^{\mathrm{a}}$} & \multicolumn{3}{|c|}{ Component 1} & \multicolumn{2}{|c|}{ Component 2} & \multirow[b]{2}{*}{$\chi^{2} /$ dof } \\
\hline & & $\begin{array}{c}k T \\
(\mathrm{keV})\end{array}$ & $\begin{array}{c}\text { abund } \\
\mathrm{Z}_{\odot}\end{array}$ & $\begin{array}{l}\text { Norm. }^{\mathrm{b}} \\
\left(\mathrm{cm}^{-5}\right)\end{array}$ & $\begin{array}{c}k T \\
(\mathrm{keV})\end{array}$ & Norm. $^{\mathrm{c}}$ & \\
\hline \multirow[t]{3}{*}{ A 3526} & $1 \mathrm{~T}$ & $3.90_{-0.05}^{+0.08}$ & $0.514_{-0.013}^{+0.009}$ & $0.1129_{-0.0035}^{+0.0014}$ & \multirow{3}{*}{3.83} & & $1485.23 / 1496$ \\
\hline & $2 \mathrm{~T}$ & 4.06 & 0.513 & 0.0559 & & 0.0552 & $1485.35 / 1494$ \\
\hline & $\mathrm{T}+\mathrm{IC}$ & $3.82_{-0.10}^{+0.03}$ & $0.528_{-0.009}^{+0.013}$ & $0.1162_{-0.0016}^{+0.0037}$ & & $<0.0012$ & $1486.66 / 1495$ \\
\hline \multirow[t]{3}{*}{ A1644 } & $1 \mathrm{~T}$ & $5.74_{-0.54}^{+0.49}$ & $0.301_{-0.049}^{+0.051}$ & $0.0411_{-0.0018}^{+0.0024}$ & \multirow{3}{*}{6.91} & & $231.49 / 305$ \\
\hline & $2 \mathrm{~T}$ & 4.47 & 0.314 & 0.0220 & & 0.0204 & $229.77 / 303$ \\
\hline & $\mathrm{T}+\mathrm{IC}$ & $5.70_{-0.60}^{+0.53}$ & $0.311_{-0.056}^{+0.062}$ & $0.0400_{-0.0047}^{+0.0034}$ & & $<0.0021$ & $230.94 / 304$ \\
\hline \multirow[t]{3}{*}{ A1650 } & $1 \mathrm{~T}$ & $6.12_{-0.25}^{+0.25}$ & $0.399_{-0.028}^{+0.029}$ & $0.0270_{-0.0007}^{+0.0007}$ & \multirow{4}{*}{7.11} & & $454.09 / 603$ \\
\hline & $2 \mathrm{~T}$ & 5.43 & 0.399 & 0.0157 & & 0.0114 & $453.63 / 601$ \\
\hline & $\mathrm{T}+\mathrm{IC}$ & $6.00_{-0.29}^{+0.35}$ & $0.440_{-0.069}^{+0.042}$ & $0.0263_{-0.0050}^{+0.0014}$ & & $<0.0020$ & $453.41 / 602$ \\
\hline \multirow[t]{3}{*}{ A1651 } & $1 \mathrm{~T}$ & $6.94_{-0.63}^{+0.62}$ & $0.411_{-0.064}^{+0.068}$ & $0.0331_{-0.0013}^{+0.0019}$ & & & 133.74/199 \\
\hline & $2 \mathrm{~T}$ & 5.43 & 0.413 & 0.0037 & \multirow[t]{2}{*}{7.12} & 0.0295 & $133.52 / 197$ \\
\hline & $\mathrm{T}+\mathrm{IC}$ & $6.58_{-0.65}^{+0.70}$ & $0.495_{-0.095}^{+0.122}$ & $0.0273_{-0.0044}^{+0.0044}$ & & $0.0022_{-0.0015}^{+0.0015}$ & $128.14 / 198$ \\
\hline \multirow[t]{3}{*}{ Coma } & $1 \mathrm{~T}$ & $8.59_{-0.14}^{+0.17}$ & $0.249_{-0.015}^{+0.015}$ & $0.2435_{-0.0019}^{+0.0021}$ & \multirow{3}{*}{8.77} & & $1454.05 / 1835$ \\
\hline & $2 \mathrm{~T}$ & 8.33 & 0.249 & 0.0910 & & 0.1528 & $1453.89 / 1833$ \\
\hline & $\mathrm{T}+\mathrm{IC}$ & $8.58_{-0.14}^{+0.18}$ & $0.247_{-0.014}^{+0.016}$ & $0.2437_{-0.0028}^{+0.0020}$ & & $<0.0009$ & $1454.15 / 1834$ \\
\hline \multirow[t]{3}{*}{ NGC5044 } & $1 \mathrm{~T}$ & $1.45_{-0.15}^{+0.18}$ & $0.644_{-0.315}^{+0.513}$ & $0.0191_{-0.0049}^{+0.0068}$ & \multirow{3}{*}{36.38} & & $181.40 / 238$ \\
\hline & $2 \mathrm{~T}$ & 1.39 & 0.685 & 0.0193 & & 0.0002 & $181.70 / 236$ \\
\hline & $\mathrm{T}+\mathrm{IC}$ & $1.37_{-0.28}^{+0.22}$ & $0.801_{-0.435}^{+1.900}$ & $0.0175_{-0.0082}^{+0.0090}$ & & $<0.0006$ & $179.81 / 237$ \\
\hline \multirow[t]{3}{*}{ A3558 } & $1 \mathrm{~T}$ & $6.22_{-0.15}^{+0.15}$ & $0.334_{-0.016}^{+0.016}$ & $0.0643_{-0.0009}^{+0.0009}$ & \multirow{3}{*}{6.74} & & $911.85 / 1133$ \\
\hline & $2 \mathrm{~T}$ & 5.95 & 0.334 & 0.0423 & & 0.0220 & $911.84 / 1131$ \\
\hline & $\mathrm{T}+\mathrm{IC}$ & $6.23_{-0.16}^{+0.14}$ & $0.335_{-0.016}^{+0.016}$ & $0.0642_{-0.0019}^{+0.0010}$ & & $<0.0007$ & $911.83 / 1132$ \\
\hline \multirow[t]{3}{*}{ A3562 } & $1 \mathrm{~T}$ & $5.42_{-0.97}^{+1.22}$ & $0.405_{-0.146}^{+0.159}$ & $0.0168_{-0.0018}^{+0.0024}$ & \multirow{4}{*}{5.40} & & $29.73 / 78$ \\
\hline & $2 \mathrm{~T}$ & 5.45 & 0.404 & 0.0081 & & 0.0087 & $29.74 / 76$ \\
\hline & $\mathrm{T}+\mathrm{IC}$ & $5.41_{-0.95}^{+1.24}$ & $0.404_{-0.147}^{+0.169}$ & $0.0168_{-0.0035}^{+0.0024}$ & & $<0.0011$ & $29.74 / 77$ \\
\hline \multirow[t]{3}{*}{ A 3571} & $1 \mathrm{~T}$ & $\begin{array}{l}7.41_{-0.19}^{+0.19} \\
\end{array}$ & $0.381_{-0.020}^{+0.021}$ & $0.1089_{-0.0013}^{+0.00013}$ & & & $1281.48 / 1551$ \\
\hline & $2 \mathrm{~T}$ & 7.25 & 0.381 & 0.0336 & \multirow[t]{2}{*}{7.48} & 0.0753 & $1281.49 / 1549$ \\
\hline & $\mathrm{T}+\mathrm{IC}$ & $7.43_{-0.18}^{+0.21}$ & $0.380_{-0.019}^{+0.022}$ & $0.1090_{-0.0023}^{+0.0012}$ & & $<0.0008$ & $1280.36 / 1550$ \\
\hline
\end{tabular}


Table 5.4-Continued

\begin{tabular}{|c|c|c|c|c|c|c|c|}
\hline \multirow[b]{2}{*}{ Name } & \multirow[b]{2}{*}{ Model $^{\mathrm{a}}$} & \multicolumn{3}{|c|}{ Component 1} & \multicolumn{2}{|c|}{ Component 2} & \multirow[b]{2}{*}{$\chi^{2} /$ dof } \\
\hline & & $\begin{array}{c}k T \\
(\mathrm{keV})\end{array}$ & $\begin{array}{c}\text { abund } \\
\mathrm{Z}_{\odot}\end{array}$ & $\begin{array}{l}\text { Norm. }^{\mathrm{b}} \\
\left(\mathrm{cm}^{-5}\right)\end{array}$ & $\begin{array}{c}k T \\
(\mathrm{keV})\end{array}$ & Norm. ${ }^{\mathrm{c}}$ & \\
\hline \multirow[t]{3}{*}{ A1795 } & $1 \mathrm{~T}$ & $5.87_{-0.12}^{+0.12}$ & $0.376_{-0.014}^{+0.014}$ & $0.0778_{-0.0009}^{+0.0009}$ & \multirow{3}{*}{6.49} & & $1344.51 / 1584$ \\
\hline & $2 \mathrm{~T}$ & 4.48 & 0.384 & 0.0253 & & 0.0534 & $1344.81 / 1582$ \\
\hline & $\mathrm{T}+\mathrm{IC}$ & $5.85_{-0.11}^{+0.12}$ & $0.376_{-0.014}^{+0.017}$ & $0.0779_{-0.0027}^{+0.0005}$ & & $<0.0009$ & $1346.83 / 1583$ \\
\hline \multirow[t]{3}{*}{ A3581 } & $1 \mathrm{~T}$ & $2.00_{-010}^{+0.11}$ & $0.419_{-0.078}^{+0.087}$ & $0.0272_{-0.0022}^{+0.0023}$ & \multirow{3}{*}{14.90} & & $200.26 / 287$ \\
\hline & $2 \mathrm{~T}$ & 1.80 & 0.488 & 0.0273 & & 0.0009 & $198.38 / 285$ \\
\hline & $\mathrm{T}+\mathrm{IC}$ & $1.85_{-0.19}^{+0.20}$ & $0.497_{-0.121}^{+0.186}$ & $0.0259_{-0.0033}^{+0.0027}$ & & $<0.0010$ & $198.27 / 286$ \\
\hline \multirow[t]{3}{*}{ MKW8 } & $1 \mathrm{~T}$ & $3.83_{-0.47}^{+0.61}$ & $0.315_{-0.096}^{+0.103}$ & $0.0121_{-0.0013}^{+0.0014}$ & \multirow{3}{*}{4.36} & & $80.81 / 129$ \\
\hline & $2 \mathrm{~T}$ & 3.42 & 0.320 & 0.0068 & & 0.0052 & $80.26 / 127$ \\
\hline & $\mathrm{T}+\mathrm{IC}$ & $3.64_{-0.86}^{+0.79}$ & $0.356_{-0.130}^{+0.334}$ & $0.0111_{-0.0037}^{+0.0023}$ & & $<0.0015$ & $80.09 / 128$ \\
\hline \multirow[t]{3}{*}{ A2029 } & $1 \mathrm{~T}$ & $8.44_{-0.28}^{+0.35}$ & $0.454_{-0.033}^{+0.034}$ & $0.0756_{-0.0014}^{+0.0014}$ & \multirow{4}{*}{8.44} & & $550.57 / 641$ \\
\hline & $2 \mathrm{~T}$ & 0.05 & 0.454 & 0.0010 & & 0.0756 & $550.57 / 639$ \\
\hline & $\mathrm{T}+\mathrm{IC}$ & $8.39_{-0.30}^{+0.33}$ & $0.484_{-0.048}^{+0.046}$ & $0.0710_{-0.0043}^{+0.0051}$ & & $<0.0033$ & $548.22 / 640$ \\
\hline \multirow[t]{3}{*}{ A2052 } & $1 \mathrm{~T}$ & $3.21_{-009}^{+0.10}$ & $0.473_{-0.031}^{+0.032}$ & $0.0451_{-0.0014}^{+0.0014}$ & & & $430.55 / 532$ \\
\hline & $2 \mathrm{~T}$ & 2.69 & 0.484 & 0.0264 & \multirow[t]{2}{*}{3.93} & 0.0193 & $428.08 / 530$ \\
\hline & $\mathrm{T}+\mathrm{IC}$ & $3.11_{-0.17}^{+0.19}$ & $0.512_{-0.066}^{+0.073}$ & $0.0434_{-0.0030}^{+0.00030}$ & & $<0.0016$ & $430.00 / 531$ \\
\hline \multirow[t]{2}{*}{ MKW3S } & $1 \mathrm{~T}$ & $3.64_{-012}^{+0.13}$ & $0.381_{-0.028}^{+0.028}$ & $0.0368_{-0.0011}^{+0.0011}$ & \multirow{3}{*}{3.81} & & $421.00 / 524$ \\
\hline & $2 \mathrm{~T}$ & 3.47 & 0.381 & & & 0.0181 & $420.93 / 522$ \\
\hline \multirow{4}{*}{ A2065 } & $\mathrm{T}+\mathrm{IC}$ & $3.57_{-022}^{+0.20}$ & $0.398_{-0.044}^{+0.060}$ & $0.0357_{-0.0034}^{+0.0022}$ & & $<0.0014$ & $420.93 / 523$ \\
\hline & $1 \mathrm{~T}$ & $6.55_{-0.75}^{-0.82}$ & $0.262_{-0.079}^{+0.044}$ & $0.0288_{-0.0017}^{+0.00020}$ & \multirow{3}{*}{6.61} & & $100.71 / 165$ \\
\hline & $2 \mathrm{~T}$ & 6.45 & 0.261 & 0.0067 & & 0.0222 & $100.36 / 163$ \\
\hline & $\mathrm{T}+\mathrm{IC}$ & $6.50_{-080}^{+0.93}$ & $0.270_{-0.084}^{+0.091}$ & $0.0280_{-0.0038}^{+0.0027}$ & & $<0.0016$ & $100.22 / 164$ \\
\hline \multirow[t]{3}{*}{ A2063 } & $1 \mathrm{~T}$ & $4.50_{-021}^{+0.23}$ & $0.339_{-0.033}^{+0.034}$ & $0.0360_{-0.0012}^{+0.0012}$ & \multirow{3}{*}{4.56} & & $437.10 / 519$ \\
\hline & $2 \mathrm{~T}$ & 4.49 & 0.344 & 0.0191 & & 0.0168 & $429.78 / 517$ \\
\hline & $\mathrm{T}+\mathrm{IC}$ & $4.48_{-0.26}^{+0.24}$ & $0.339_{-0.034}^{+0.055}$ & $0.0359_{-0.0042}^{+0.0014}$ & & $<0.0015$ & $437.14 / 518$ \\
\hline \multirow[t]{3}{*}{ A2142 } & $1 \mathrm{~T}$ & $10.31_{-0.99}^{+0.99}$ & $0.193_{-0.302}^{+0.334}$ & $0.0647^{+0.00060}$ & \multirow{3}{*}{64.00} & & $27.81 / 60$ \\
\hline & $2 \mathrm{~T}$ & 8.74 & 0.205 & 0.0636 & & 0.0039 & $22.24 / 58$ \\
\hline & $\mathrm{T}+\mathrm{IC}$ & $9.14_{-1.22}^{+1.19}$ & $<0.548$ & $0.0578_{-0.0072}^{+0.0072}$ & & $0.0031_{-0.0021}^{+0.0020}$ & $21.99 / 59$ \\
\hline
\end{tabular}


Table 5.4-Continued

\begin{tabular}{|c|c|c|c|c|c|c|c|}
\hline \multirow[b]{2}{*}{ Name } & \multirow[b]{2}{*}{ Model $^{\mathrm{a}}$} & \multicolumn{3}{|c|}{ Component 1} & \multicolumn{2}{|c|}{ Component 2} & \multirow[b]{2}{*}{$\chi^{2} /$ dof } \\
\hline & & $\begin{array}{c}k T \\
(\mathrm{keV})\end{array}$ & $\begin{array}{c}\text { abund } \\
\mathrm{Z}_{\odot}\end{array}$ & $\begin{array}{l}\text { Norm. }^{b} \\
\left(\mathrm{~cm}^{-5}\right)\end{array}$ & $\begin{array}{c}k T \\
(\mathrm{keV})\end{array}$ & Norm. $^{\mathrm{c}}$ & \\
\hline \multirow[t]{3}{*}{ A 2147} & $1 \mathrm{~T}$ & $5.62_{-0.86}^{+1.14}$ & $0.239_{-0.128}^{+0.138}$ & $0.0381_{-0.0037}^{+0.0044}$ & \multirow{3}{*}{5.72} & & $56.40 / 99$ \\
\hline & $2 \mathrm{~T}$ & 5.55 & 0.239 & 0.0234 & & 0.0147 & $56.39 / 97$ \\
\hline & $\mathrm{T}+\mathrm{IC}$ & $5.51_{-0.79}^{+1.24}$ & $0.236_{-0.124}^{+0.140}$ & $0.0383_{-0.0043}^{+0.0042}$ & & $<0.0011$ & $56.48 / 98$ \\
\hline \multirow[t]{3}{*}{ A 2199} & $1 \mathrm{~T}$ & $4.59_{-0.13}^{+0.14}$ & $0.367_{-0.021}^{+0.022}$ & $0.0994_{-0.0021}^{+0.0021}$ & \multirow{3}{*}{4.82} & & $573.12 / 746$ \\
\hline & $2 \mathrm{~T}$ & 4.37 & 0.367 & 0.0498 & & 0.0496 & $573.18 / 744$ \\
\hline & $\mathrm{T}+\mathrm{IC}$ & $4.57_{-0.14}^{+0.16}$ & $0.371_{-0.025}^{+0.026}$ & $0.0983_{-0.0039}^{+0.0031}$ & & $<0.0017$ & $572.83 / 745$ \\
\hline \multirow[t]{3}{*}{ A 2204} & $1 \mathrm{~T}$ & $7.46_{-0.32}^{+0.33}$ & $0.414_{-0.032}^{+0.033}$ & $0.0453_{-0.0010}^{+0.0011}$ & \multirow{3}{*}{15.36} & & $375.74 / 507$ \\
\hline & $2 \mathrm{~T}$ & 5.44 & 0.458 & 0.0308 & & 0.0160 & $366.90 / 505$ \\
\hline & $\mathrm{T}+\mathrm{IC}$ & $7.36_{-0.36}^{+0.34}$ & $0.433_{-0.048}^{+0.073}$ & $0.0433_{-0.0058}^{+0.0031}$ & & $<0.0025$ & $377.80 / 506$ \\
\hline \multirow[t]{3}{*}{ A 2256} & $1 \mathrm{~T}$ & $7.90_{-0.47}^{+0.49}$ & $0.333_{-0.051}^{+0.054}$ & $0.0493_{-0.0015}^{+0.0015}$ & \multirow{3}{*}{7.93} & & $192.32 / 262$ \\
\hline & $2 \mathrm{~T}$ & 0.03 & 0.337 & 0.0000 & & 0.0494 & $191.67 / 260$ \\
\hline & $\mathrm{T}+\mathrm{IC}$ & $7.84_{-0.51}^{+0.50}$ & $0.333_{-0.053}^{+0.057}$ & $0.0491_{-0.0035}^{+0.0017}$ & & $<0.0014$ & $194.33 / 261$ \\
\hline \multirow[t]{3}{*}{ A 2255} & $1 \mathrm{~T}$ & $7.18_{-0.86}^{+1.13}$ & $0.256_{-0.100}^{+0.108}$ & $0.0242_{-0.0016}^{+0.0017}$ & \multirow{3}{*}{7.47} & & $55.91 / 119$ \\
\hline & $2 \mathrm{~T}$ & 6.94 & 0.255 & 0.0132 & & 0.0110 & $55.91 / 117$ \\
\hline & $\mathrm{T}+\mathrm{IC}$ & $7.32_{-1.01}^{+1.01}$ & $0.257_{-0.103}^{+0.107}$ & $0.0240_{-0.0018}^{+0.0019}$ & & $<0.0006$ & $55.90 / 118$ \\
\hline \multirow[t]{3}{*}{ A 3667} & $1 \mathrm{~T}$ & $7.17_{-0.20}^{+0.21}$ & $0.276_{-0.016}^{+0.017}$ & $\begin{array}{l}0.0729_{-0.0009}^{+0.0009} \\
\end{array}$ & \multirow{3}{*}{7.76} & & $1146.80 / 1320$ \\
\hline & $2 \mathrm{~T}$ & 6.62 & 0.278 & 0.0370 & & 0.0361 & $1146.61 / 1318$ \\
\hline & $\mathrm{T}+\mathrm{IC}$ & $7.21_{-0.21}^{+0.21}$ & $0.272_{-0.016}^{+0.016}$ & $0.0729_{-0.0041}^{+0.0009}$ & & $<0.0015$ & $1154.85 / 1319$ \\
\hline \multirow[t]{3}{*}{ S1101 } & $1 \mathrm{~T}$ & $2.85_{-0.13}^{+0.14}$ & $0.335_{-0.042}^{+0.040}$ & $0.0236_{-0.0012}^{+0.0012}$ & \multirow{3}{*}{4.09} & & $213.81 / 283$ \\
\hline & $2 \mathrm{~T}$ & 2.16 & 0.356 & 0.0176 & & 0.0079 & $212.32 / 281$ \\
\hline & $\mathrm{T}+\mathrm{IC}$ & $2.85_{-0.22}^{+0.14}$ & $0.334_{-0.039}^{+0.056}$ & $0.0237_{-0.0015}^{+0.0012}$ & & $<0.0005$ & $213.82 / 282$ \\
\hline \multirow[t]{3}{*}{ A 2589} & $1 \mathrm{~T}$ & $3.86_{-0.21}^{+0.23}$ & $0.546_{-0.053}^{+0.055}$ & $\begin{array}{r}0.0197_{-0.0009}^{+0.0009} \\
\end{array}$ & \multirow{4}{*}{3.86} & & $163.48 / 252$ \\
\hline & $2 \mathrm{~T}$ & 0.01 & 0.546 & 0.0013 & & 0.0197 & $163.48 / 250$ \\
\hline & $\mathrm{T}+\mathrm{IC}$ & $3.32_{-0.30}^{+0.55}$ & $0.830_{-0.263}^{+0.253}$ & $0.0145_{-0.0023}^{+0.0045}$ & & $0.0017_{-0.0014}^{+0.0006}$ & $159.07 / 250$ \\
\hline \multirow[t]{3}{*}{ A 2597} & $1 \mathrm{~T}$ & $3.89_{-0.16}^{+0.17}$ & $0.316_{-0.025}^{+0.025}$ & $0.0238_{-0.0009}^{+0.0009}$ & & & $310.40 / 407$ \\
\hline & $2 \mathrm{~T}$ & 3.44 & 0.314 & 0.0128 & \multirow[t]{2}{*}{4.52} & 0.0109 & $311.02 / 405$ \\
\hline & $\mathrm{T}+\mathrm{IC}$ & $3.79_{-0.31}^{+0.27}$ & $0.335_{-0.044}^{+0.061}$ & $0.0228_{-0.0026}^{+0.0019}$ & & $<0.0011$ & $310.02 / 406$ \\
\hline
\end{tabular}


Table 5.4-Continued

\begin{tabular}{|c|c|c|c|c|c|c|c|}
\hline \multirow[b]{2}{*}{ Name } & \multirow[b]{2}{*}{ Model $^{\mathrm{a}}$} & \multicolumn{3}{|c|}{ Component 1} & \multicolumn{2}{|c|}{ Component 2} & \multirow[b]{2}{*}{$\chi^{2} /$ dof } \\
\hline & & $\begin{array}{c}k T \\
(\mathrm{keV})\end{array}$ & $\begin{array}{c}\text { abund } \\
\mathrm{Z}_{\odot}\end{array}$ & $\begin{array}{l}\text { Norm. }^{b} \\
\left(\mathrm{~cm}^{-5}\right)\end{array}$ & $\begin{array}{c}k T \\
(\mathrm{keV})\end{array}$ & Norm. $^{\mathrm{c}}$ & \\
\hline \multirow[t]{3}{*}{ A 2634} & $1 \mathrm{~T}$ & $4.74_{-0.87}^{+1.16}$ & $0.284_{-0.143}^{+0.151}$ & $0.0179_{-0.0022}^{+0.0028}$ & \multirow{3}{*}{5.90} & & $62.84 / 79$ \\
\hline & $2 \mathrm{~T}$ & 0.60 & 0.299 & 0.0804 & & 0.0150 & $61.17 / 77$ \\
\hline & $\mathrm{T}+\mathrm{IC}$ & $4.79_{-0.89}^{+1.16}$ & $0.279_{-0.139}^{+0.140}$ & $0.0179_{-0.0028}^{+0.0029}$ & & $<0.0008$ & $62.74 / 78$ \\
\hline \multirow[t]{3}{*}{ A 2657} & $1 \mathrm{~T}$ & $5.73_{-0.60}^{+0.64}$ & $0.251_{-0.066}^{+0.068}$ & $0.0242_{-0.0013}^{+0.0016}$ & \multirow{3}{*}{6.22} & & $176.95 / 235$ \\
\hline & $2 \mathrm{~T}$ & 4.34 & 0.255 & 0.0063 & & 0.0181 & $176.75 / 233$ \\
\hline & $\mathrm{T}+\mathrm{IC}$ & $5.79_{-0.66}^{+0.59}$ & $0.252_{-0.067}^{+0.068}$ & $0.0241_{-0.0021}^{+0.0017}$ & & $<0.0007$ & $176.95 / 234$ \\
\hline \multirow[t]{3}{*}{ A 4038} & $1 \mathrm{~T}$ & $3.39_{-0.11}^{+0.12}$ & $0.346_{-0.025}^{+0.026}$ & $0.0559_{-0.0017}^{+0.0019}$ & \multirow{3}{*}{3.74} & & $579.52 / 726$ \\
\hline & $2 \mathrm{~T}$ & 3.06 & 0.351 & 0.0301 & & 0.0264 & $579.27 / 724$ \\
\hline & $\mathrm{T}+\mathrm{IC}$ & $3.36_{-0.18}^{+0.14}$ & $0.351_{-0.031}^{+0.045}$ & $0.0554_{-0.0032}^{+0.0024}$ & & $<0.0013$ & $579.42 / 725$ \\
\hline \multirow[t]{3}{*}{ A 4059} & $1 \mathrm{~T}$ & $4.43_{-0.21}^{+0.23}$ & $0.428_{-0.036}^{+0.037}$ & $0.0330_{-0.0011}^{+0.0012}$ & \multirow{3}{*}{4.45} & & $259.33 / 427$ \\
\hline & $2 \mathrm{~T}$ & 4.38 & 0.428 & 0.0118 & & 0.0211 & $259.34 / 425$ \\
\hline & $\mathrm{T}+\mathrm{IC}$ & $4.41_{-0.19}^{+0.25}$ & $0.426_{-0.034}^{+0.039}$ & $0.0331_{-0.0017}^{+0.0011}$ & & $<0.0005$ & $259.36 / 426$ \\
\hline
\end{tabular}

aParameters for the $2 \mathrm{~T}$ model are unconstrained.

${ }^{b}$ Normalization of the APEC thermal spectrum, which is given by $\left\{10^{-14} /[4 \pi(1+\right.$ $\left.\left.z)^{2} D_{A}^{2}\right]\right\} \int n_{e} n_{H} d V$, where $z$ is the redshift, $D_{A}$ is the angular diameter distance, $n_{e}$ is the electron density, $n_{H}$ is the ionized hydrogen density, and $V$ is the volume of the cluster.

${ }^{c}$ Value is the normalization of the power-law component for the $\mathrm{T}+\mathrm{IC}$ model, which is the photon flux at a photon energy of $1 \mathrm{keV}$ in units of photons $\mathrm{cm}^{-2} \mathrm{~s}^{-1} \mathrm{keV}^{-1}$. For the $2 \mathrm{~T}$ model, the value is the normalization of the second APEC thermal model in units of $\mathrm{cm}^{-5}$. 
Table 5.5. Upper Limits to 20-80 keV Nonthermal Flux from EPIC and BAT Joint Fits

\begin{tabular}{|c|c|c|c|c|c|c|c|c|}
\hline \multirow[b]{2}{*}{ Name } & \multicolumn{4}{|c|}{ Spectral Band: 2-195 keV } & \multicolumn{4}{|c|}{ Spectral Band: 3-195 keV } \\
\hline & $90 \%^{a}$ & $3 \sigma_{\Gamma=2}^{\mathrm{a}}$ & $\Gamma$ & $3 \sigma^{\mathrm{a}}$ & $90 \%^{\mathrm{a}}$ & $3 \sigma_{\Gamma=2}^{\mathrm{a}}$ & $\Gamma$ & $3 \sigma^{\mathrm{a}}$ \\
\hline A0085 & 4.33 & 6.58 & 2.00 & 6.58 & 0.82 & 4.02 & 1.19 & 5.32 \\
\hline A0119 & 4.28 & 6.65 & 2.13 & 6.38 & 1.61 & 4.55 & 2.65 & 1.24 \\
\hline A0133 & 5.43 & 7.38 & 2.06 & 5.82 & 4.08 & 7.58 & 2.11 & 5.50 \\
\hline NGC507 & 1.54 & 1.73 & 2.00 & 2.15 & 1.45 & 1.84 & 1.92 & 2.34 \\
\hline A0262 & 3.29 & 4.08 & 2.01 & 4.03 & 0.44 & 2.62 & 9.50 & $1.3 \times 10^{-7}$ \\
\hline A0400 & 1.35 & 2.26 & 6.26 & $2.1 \times 10^{-5}$ & 0.67 & 2.22 & 9.34 & $2.2 \times 10^{-7}$ \\
\hline A0399 & 1.72 & 4.20 & 3.25 & 0.11 & -0.15 & 3.81 & 9.50 & $1.4 \times 10^{-7}$ \\
\hline A3112 & 7.19 & 9.09 & 2.00 & 8.98 & 5.43 & 7.23 & 2.16 & 7.06 \\
\hline Fornax & 3.19 & 3.13 & 2.00 & 3.13 & 3.25 & 3.07 & 2.18 & 2.94 \\
\hline $2 \mathrm{~A} 0335$ & 4.44 & 6.68 & 2.08 & 6.57 & 2.11 & 4.56 & 9.38 & $6.8 \times 10^{-7}$ \\
\hline IIIZw54 & 3.68 & 4.33 & 2.03 & 4.15 & 2.59 & 5.56 & 2.21 & 3.24 \\
\hline A3158 & 3.18 & 5.08 & 1.94 & 5.22 & 3.45 & 4.51 & 1.99 & 4.53 \\
\hline NGC1550 & 1.83 & 1.92 & 2.06 & 1.94 & 2.54 & 2.99 & 2.39 & 1.57 \\
\hline EXO0422 & 4.42 & 5.27 & 2.01 & 5.14 & 3.93 & 5.11 & 2.03 & 4.98 \\
\hline A3266 & 6.54 & 8.43 & 2.00 & 8.43 & 6.19 & 8.58 & 2.00 & 8.58 \\
\hline A0496 & 4.28 & 7.04 & 2.00 & 6.27 & 0.35 & 3.28 & 2.11 & 3.33 \\
\hline A3376 & 5.18 & 5.31 & 2.10 & 4.87 & 4.91 & 5.90 & 2.02 & 5.78 \\
\hline A3391 & 4.25 & 6.11 & 2.00 & 6.10 & 3.91 & 6.35 & 2.00 & 6.03 \\
\hline A3395s & 4.21 & 2.89 & 2.18 & 2.32 & 5.00 & 3.89 & 2.13 & 3.76 \\
\hline A 0576 & 4.50 & 6.50 & 2.00 & 6.41 & 4.01 & 6.39 & 2.02 & 6.36 \\
\hline A0754 & 7.31 & 9.14 & 2.08 & 7.60 & 5.38 & 8.68 & 1.79 & 10.35 \\
\hline HydraA & 6.64 & 8.72 & 2.15 & 8.30 & 3.97 & 5.83 & 2.08 & 5.71 \\
\hline A1060 & 6.09 & 7.30 & 2.02 & 7.29 & 0.94 & 3.25 & 2.60 & 0.75 \\
\hline A1367 & 6.88 & 8.39 & 2.01 & 8.36 & 5.48 & 7.36 & 2.00 & 7.39 \\
\hline MKW4 & 1.06 & 2.66 & 1.99 & 2.00 & 2.05 & 2.53 & 1.77 & 3.52 \\
\hline ZwCl1215 & 3.81 & 5.57 & 2.00 & 5.53 & 0.96 & 4.15 & 2.26 & 2.46 \\
\hline
\end{tabular}


Table 5.5-Continued

\begin{tabular}{|c|c|c|c|c|c|c|c|c|}
\hline \multirow[b]{2}{*}{ Name } & \multicolumn{4}{|c|}{ Spectral Band: 2-195 keV } & \multicolumn{4}{|c|}{ Spectral Band: 3-195 keV } \\
\hline & $90 \%^{\mathrm{a}}$ & $3 \sigma_{\Gamma=2}^{\mathrm{a}}$ & $\Gamma$ & $3 \sigma^{\mathrm{a}}$ & $90 \%{ }^{\mathrm{a}}$ & $3 \sigma_{\Gamma=2}^{\mathrm{a}}$ & $\Gamma$ & $3 \sigma^{\mathrm{a}}$ \\
\hline NGC4636 & 0.57 & 0.62 & 1.99 & 0.64 & 0.95 & 0.90 & 2.00 & 0.89 \\
\hline A 3526 & 12.86 & 8.91 & 2.00 & 8.90 & 15.21 & 6.71 & 2.18 & 5.27 \\
\hline A1644 & 5.97 & 8.66 & 2.01 & 8.61 & 5.06 & 7.84 & 2.10 & 7.46 \\
\hline A1650 & 4.99 & 6.63 & 2.00 & 6.62 & 4.31 & 6.71 & 2.00 & 6.71 \\
\hline A1651 & 8.83 & 10.82 & 2.00 & 10.82 & 8.20 & 10.88 & 1.96 & 11.09 \\
\hline Coma & 4.35 & 5.92 & 2.00 & 5.92 & 1.57 & 4.65 & 2.01 & 4.63 \\
\hline NGC5044 & 1.69 & 2.40 & 2.00 & 1.91 & 1.62 & 2.11 & 2.22 & 1.34 \\
\hline A3558 & 6.96 & 10.61 & 2.53 & 1.83 & 0.36 & 3.93 & 2.27 & 1.46 \\
\hline A3562 & 2.69 & 5.53 & 8.01 & $2.1 \times 10^{-7}$ & 2.39 & 5.48 & 7.65 & $1.2 \times 10^{-5}$ \\
\hline A3571 & 6.07 & 8.52 & 2.39 & 3.51 & 0.33 & 4.38 & 9.45 & $1.4 \times 10^{-7}$ \\
\hline A1795 & 4.82 & 7.22 & 2.71 & 0.98 & 1.83 & 3.96 & 2.11 & 2.92 \\
\hline A3581 & 2.65 & 2.76 & 2.00 & 2.75 & 2.35 & 3.73 & 2.34 & 1.67 \\
\hline MKW8 & 5.30 & 5.00 & 2.05 & 4.76 & 3.51 & 5.23 & 2.03 & 5.04 \\
\hline A 2029 & 10.84 & 13.33 & 2.00 & 13.32 & 8.03 & 10.51 & 1.82 & 11.50 \\
\hline A2052 & 5.81 & 6.76 & 2.01 & 6.30 & 3.87 & 5.00 & 2.07 & 4.99 \\
\hline MKW3S & 4.69 & 6.01 & 2.00 & 5.96 & 14.08 & 5.14 & 1.95 & 5.34 \\
\hline A2065 & 3.48 & 5.59 & 2.00 & 5.60 & 3.75 & 5.93 & 2.00 & 5.93 \\
\hline A2063 & 4.85 & 6.58 & 2.18 & 5.34 & 3.02 & 5.81 & 2.17 & 4.95 \\
\hline A 2142 & 10.34 & 13.36 & 2.00 & 13.21 & 11.27 & 14.84 & 1.98 & 14.65 \\
\hline $\mathrm{A} 2147$ & 2.82 & 5.51 & 2.01 & 5.46 & 2.14 & 2.60 & 9.50 & $3.5 \times 10^{-7}$ \\
\hline A2199 & 5.35 & 7.75 & 2.20 & 8.05 & 3.80 & 6.09 & 2.17 & 5.89 \\
\hline A2204 & 9.04 & 11.24 & 2.09 & 9.30 & 5.01 & 8.92 & 2.05 & 8.11 \\
\hline A 2256 & 6.48 & 8.45 & 2.23 & 6.36 & 3.18 & 5.03 & 2.17 & 2.41 \\
\hline $\mathrm{A} 2255$ & 0.55 & 2.77 & 6.67 & $7.9 \times 10^{-6}$ & 0.51 & 3.34 & 7.09 & $1.6 \times 10^{-5}$ \\
\hline A3667 & 11.40 & 13.64 & 2.03 & 13.33 & 27.98 & 6.43 & 2.02 & 6.19 \\
\hline S1101 & 1.57 & 2.27 & 2.20 & 1.90 & 1.81 & 2.28 & 2.19 & 1.92 \\
\hline
\end{tabular}


Table 5.5-Continued

\begin{tabular}{|c|c|c|c|c|c|c|c|c|}
\hline \multirow[b]{2}{*}{ Name } & \multicolumn{4}{|c|}{ Spectral Band: 2-195 keV } & \multicolumn{4}{|c|}{ Spectral Band: 3-195 keV } \\
\hline & $90 \% \mathrm{a}$ & $3 \sigma_{\Gamma=2}^{\mathrm{a}}$ & $\Gamma$ & $3 \sigma^{\mathrm{a}}$ & $90 \%{ }^{\mathrm{a}}$ & $3 \sigma_{\Gamma=2^{\mathrm{a}}}$ & $\Gamma$ & $3 \sigma^{\mathrm{a}}$ \\
\hline A 2589 & 4.77 & 6.02 & 2.00 & 6.00 & 5.07 & 6.73 & 2.04 & 6.43 \\
\hline A 2597 & 5.75 & 6.10 & 2.00 & 6.09 & 2.26 & 4.31 & 2.35 & 3.08 \\
\hline A 2634 & 3.83 & 4.36 & 7.24 & $3.8 \times 10^{-6}$ & 1.52 & 4.02 & 6.68 & $1.6 \times 10^{-4}$ \\
\hline $\mathrm{A} 2657$ & 2.28 & 4.58 & 2.04 & 4.45 & 0.87 & 3.96 & 9.50 & $2.6 \times 10^{-7}$ \\
\hline A 4038 & 6.01 & 7.69 & 2.00 & 7.48 & 3.14 & 4.92 & 2.14 & 4.54 \\
\hline A 4059 & 2.00 & 3.79 & 8.31 & $1.8 \times 10^{-7}$ & -0.14 & 2.70 & 9.50 & $1.4 \times 10^{-7}$ \\
\hline
\end{tabular}

${ }^{\mathrm{a}} 20-80 \mathrm{keV}, 10^{-12} \mathrm{erg}^{-2} \mathrm{~s}^{-1}$ 
Table 5.6. Fits to Stacked EPIC and BAT Spectra

\begin{tabular}{|c|c|c|c|c|c|c|c|}
\hline \multirow{2}{*}{$\begin{array}{l}\text { Sample } \\
\text { (number) }\end{array}$} & \multirow[b]{2}{*}{$\mathrm{T}_{\text {Model }}$} & \multirow{2}{*}{$\begin{array}{c}\text { T}_{\text {Model }} \text {-only } \\
\chi^{2} / \text { dof }\end{array}$} & \multicolumn{2}{|c|}{$\mathrm{T}_{\text {Model }}+\mathrm{IC}$} & \multicolumn{3}{|c|}{$\mathrm{T}_{\text {Model }}+\mathrm{IC}, \Gamma_{\text {free }}$} \\
\hline & & & Norm. $^{\mathrm{a}}$ & $\chi^{2} /$ dof & $\Gamma$ & Norm. $^{\mathrm{a}}$ & $\chi^{2} /$ dof \\
\hline All & $1 \mathrm{~T}_{\mathrm{X},>2}$ & $1263.33 / 1746$ & $0.0107_{-0.0099}^{+0.0099}$ & $1260.13 / 1745$ & 7.61 & $1.5078_{-0.2629}^{+0.2629}$ & $29 / 1745$ \\
\hline \multirow[t]{3}{*}{$(58)$} & $1 \mathrm{~T}_{\mathrm{X},>3}$ & $1009.16 / 1546$ & $<0.0059$ & $1009.16 / 1545$ & 1.00 & $<0.0001$ & $1009.16 / 1545$ \\
\hline & $1 \mathrm{~T}_{\mathrm{J}}$ & $1274.36 / 1746$ & $<0.0196$ & $1271.72 / 1745$ & 7.58 & $1.5343_{-0.2576}^{+0.2576}$ & $1178.39 / 1745$ \\
\hline & $2 \mathrm{~T}_{\mathrm{J}}$ & $1268.44 / 1746$ & $<0.0077$ & $1268.45 / 1745$ & 1.00 & $<0.0001$ & $1268.45 / 1745$ \\
\hline Hot & $1 \mathrm{~T}_{\mathrm{X},>2}$ & $996.72 / 1746$ & $0.0071_{-0.0046}^{+0.0046}$ & $990.30 / 1745$ & 2.15 & $0.0100_{-0.0061}^{+0.0061}$ & $989.35 / 1745$ \\
\hline \multirow[t]{3}{*}{ (11) } & $1 \mathrm{~T}_{\mathrm{X},>3}$ & $826.03 / 1546$ & $<0.0070$ & $825.62 / 1545$ & 0.95 & $<0.0$ & $824.64 / 1545$ \\
\hline & $1 \mathrm{~T}_{\mathrm{J}}$ & $998.54 / 1746$ & $0.0064_{-0.0046}^{+0.0047}$ & $993.40 / 1745$ & 0.99 & $<0.0002$ & $996.65 / 1745$ \\
\hline & $2 \mathrm{~T}_{\mathrm{J}}$ & $994.29 / 1746$ & $<0.0053$ & $5 / 1745$ & 0.03 & $<0.0000$ & $994.09 / 1745$ \\
\hline Cool & $1 \mathrm{~T}_{\mathrm{X},>2}$ & $1186.10 / 1746$ & $<0.0078$ & $1186.09 / 1745$ & 1.00 & $<0.0$ & $1186.10 / 1745$ \\
\hline \multirow[t]{3}{*}{$(47)$} & $1 \mathrm{~T}_{\mathrm{X},>3}$ & $976.64 / 1546$ & $<0.0040$ & $976.64 / 1545$ & 1.00 & $<0.0$ & $976.64 / 1545$ \\
\hline & $1 \mathrm{~T}_{\mathrm{J}}$ & $1192.30 / 1746$ & $<0.0080$ & $1192.29 / 1745$ & 1.00 & $<0.0$ & $1192.30 / 1745$ \\
\hline & $2 \mathrm{~T}_{\mathrm{J}}$ & $1187.00 / 1746$ & $<0.0037$ & $1187.00 / 1745$ & 1.00 & $<0.0001$ & $1187.00 / 1745$ \\
\hline Radio & $1 \mathrm{~T}_{\mathrm{X},>2}$ & $951.17 / 1746$ & $0.0088_{-0.0041}^{+0.0041}$ & $939.02 / 1745$ & 2.10 & $0.0114_{-0.0052}^{+0.0052}$ & $938.14 / 1745$ \\
\hline \multirow[t]{3}{*}{ (9) } & $1 \mathrm{~T}_{\mathrm{X},>3}$ & $825.05 / 1546$ & $0.0064_{-0.0045}^{+0.0045}$ & $819.52 / 1545$ & 2.13 & $0.0103_{-0.0071}^{+0.0071}$ & $819.29 / 1545$ \\
\hline & & $948.97 / 1746$ & $0.0079_{-0.0042}^{+0.0042}$ & $939.28 / 1745$ & 2.13 & $0.0112_{-0.0055}^{+0.0055}$ & $937.92 / 1745$ \\
\hline & $2 \mathrm{~T}_{\mathrm{J}}$ & $943.85 / 1746$ & $<0.0079$ & $941.84 / 1745$ & 1.09 & $<0.0001$ & $943.70 / 1745$ \\
\hline No Radio & $1 \mathrm{~T}_{\mathrm{X},>2}$ & $1236.64 / 1746$ & $<0.0105$ & $1236.48 / 1745$ & 7.08 & $0.6788_{-0.1544}^{+0.1544}$ & $1184.35 / 1745$ \\
\hline
\end{tabular}


Table 5.6-Continued

\begin{tabular}{|c|c|c|c|c|c|c|c|}
\hline \multirow{2}{*}{$\begin{array}{l}\text { Sample } \\
\text { (number) }\end{array}$} & \multirow[b]{2}{*}{$\mathrm{T}_{\text {Model }}$} & \multirow{2}{*}{$\begin{array}{c}\mathrm{T}_{\text {Model }} \text {-only } \\
\chi^{2} / \text { dof }\end{array}$} & \multicolumn{2}{|c|}{$\mathrm{T}_{\text {Model }}+\mathrm{IC}$} & \multicolumn{3}{|c|}{$\mathrm{T}_{\text {Model }}+\mathrm{IC}, \Gamma_{\text {free }}$} \\
\hline & & & Norm. $^{\text {a }}$ & $\chi^{2} /$ dof & $\Gamma$ & Norm. $^{\text {a }}$ & $\chi^{2} /$ dof \\
\hline \multirow[t]{3}{*}{$(49)$} & $1 \mathrm{~T}_{\mathrm{X},>3}$ & $1011.03 / 1546$ & $<0.0036$ & $1011.03 / 1545$ & 1.00 & $<0.0001$ & $1011.03 / 1545$ \\
\hline & $1 \mathrm{~T}_{\mathrm{J}}$ & $1242.35 / 1746$ & $<0.0107$ & $1242.15 / 1745$ & 3.60 & $0.0632_{-0.0166}^{+0.0166}$ & $1203.18 / 1745$ \\
\hline & $2 \mathrm{~T}_{\mathrm{J}}$ & $1236.13 / 1746$ & $<0.0044$ & $1236.13 / 1745$ & 1.00 & $<0.0001$ & $1236.13 / 1745$ \\
\hline $\mathrm{NCC}$ & $1 \mathrm{~T}_{\mathrm{X},>2}$ & $957.56 / 1746$ & $0.0070_{-0.0052}^{+0.0052}$ & $952.65 / 1745$ & 6.81 & $0.1996_{-0.0847}^{+0.0847}$ & $942.52 / 1745$ \\
\hline \multirow[t]{3}{*}{$(16)$} & $1 \mathrm{~T}_{\mathrm{X},>3}$ & $813.64 / 1546$ & $<0.0044$ & $813.64 / 1545$ & 1.00 & $<0.0001$ & $813.64 / 1545$ \\
\hline & $1 \mathrm{~T}_{\mathrm{J}}$ & $956.75 / 1746$ & $0.0065_{-0.0052}^{+0.0053}$ & $952.54 / 1745$ & 2.33 & $0.0134_{-0.0077}^{+0.0077}$ & $948.49 / 1745$ \\
\hline & $2 \mathrm{~T}_{\mathrm{J}}$ & $951.27 / 1746$ & $<0.0072$ & $951.19 / 1745$ & 1.00 & $<0.0001$ & $951.27 / 1745$ \\
\hline WCC & $1 \mathrm{~T}_{\mathrm{X},>2}$ & $853.23 / 1746$ & $0.0063_{-0.0057}^{+0.0057}$ & $849.86 / 1745$ & 0.60 & $<0.0000$ & $853.05 / 1745$ \\
\hline \multirow[t]{3}{*}{$(17)$} & $1 \mathrm{~T}_{\mathrm{X},>3}$ & $698.91 / 1546$ & $<0.0068$ & $698.83 / 1545$ & 0.85 & $<0.0001$ & $698.51 / 1545$ \\
\hline & $1 \mathrm{~T}_{\mathrm{J}}$ & $852.85 / 1746$ & $<0.0113$ & $850.24 / 1745$ & 0.51 & $<0.0000$ & $852.72 / 1745$ \\
\hline & $2 \mathrm{~T}_{\mathrm{J}}$ & $855.05 / 1746$ & $<0.0083$ & $854.54 / 1745$ & 0.44 & $<0.0000$ & $855.05 / 1745$ \\
\hline $\mathrm{SCC}$ & $1 \mathrm{~T}_{\mathrm{X},>2}$ & $1436.76 / 1746$ & $0.0053_{-0.0046}^{+0.0053}$ & $1433.22 / 1745$ & 2.19 & $0.0109_{-0.0082}^{+0.0082}$ & $1432.06 / 1745$ \\
\hline \multirow[t]{3}{*}{$(25)$} & $1 \mathrm{~T}_{\mathrm{X},>3}$ & $1212.36 / 1546$ & $<0.0054$ & $1212.36 / 1545$ & 1.00 & $<0.0001$ & $1212.36 / 1545$ \\
\hline & $1 T_{J}$ & $1439.49 / 1746$ & $<0.0095$ & $1437.28 / 1745$ & 2.33 & $0.0136_{-0.0094}^{+0.0094}$ & $1433.82 / 1745$ \\
\hline & $2 \mathrm{~T}_{\mathrm{J}}$ & $1431.32 / 1746$ & $<0.0053$ & $1431.30 / 1745$ & 1.00 & $<0.0001$ & $1431.32 / 1745$ \\
\hline
\end{tabular}

${ }^{\mathrm{a}}$ At a photon energy of $1 \mathrm{keV}$ in units of photons $\mathrm{cm}^{-2} \mathrm{~s}^{-1} \mathrm{keV}^{-1}$. 


\section{Chapter 6}

\section{Summary and Future Prospects}

\subsection{The Effect of Mergers on the SZ Properties of Clusters, and Cosmological Studies with SZ Cluster Samples}

In Chapter 2, we explored the impact galaxy cluster mergers have on the measured SZ properties of clusters of galaxies. Two SZ observables were followed over the course of a handful of binary cluster merger simulations and were seen to exhibit "boosts" to their intensity. For the central or maximum Comptonization parameter, $y_{\max }$, this boost was comparable in size to similar boosts in X-ray temperature and luminosity noticed in the same simulations (Ricker \& Sarazin 2001). As expected, these results agree since $y_{\max }$ is dominated by the high pressures in cluster cores, while the X-ray luminosity and temperature is also dominated by the densest gas due to the density squared dependence of X-ray emission. Two SZ observables, representing extreme cases, were followed over the course of a handful of binary cluster merger simulations and were seen to exhibit "boosts" to their intensity. while X-ray quantities also 
over-represent the conditions in the cores,

On the other hand, the boosts to the integrated Comptonization parameter $Y$ are much smaller. Because merger shocks take a finite time - hundreds of millions of years - to cross the entire cluster, the increase in the value of $Y$ is more gradual and less pronounced as some regions are compressed (increasing $Y$ ) while others are expanding (decreasing $Y$ ). The cluster is basically ringing during the merging process. Also, the maximum boost in $Y$ never exceeds the increase in the final equilibrium value, which represents the thermalization of the kinetic energy of the merger.

We also assessed the effect that cluster mergers have on the ability of SZ surveys to accurately construct mass functions, which can be used to measure cosmological parameters such as $\Omega_{M}, \sigma_{8}$, and $\Omega_{\Lambda}$. Because of the large merger boost, the use of the use of $y_{\max }$ (or any similar central SZ observable), $T_{X}$, and $L_{X}$ lead to biased estimates of cosmological parameter values (Randall et al. 2002; Wik et al. 2008). On the other extreme, integrating $y$ over the entire solid angle of the cluster (yielding $Y$ ) leads to a better mass proxy. As two clusters become one, the value of $Y$ varies between the initial and final values, which means that the inferred mass will not be overestimated and lead to a biased mass function. The lack of a systematic bias in $Y$ leaves open the possibility that SZ surveys will not only be able to accurately measure $\Omega_{M}$ and $\sigma_{8}$, but will be able to determine the evolution of the dark energy equation of state over cosmic time.

In practice, however, SZ surveys will not measure either of these quantities exactly. Most surveys will determine an integrated Comptonization parameter out to some predefined radius, dependent on the sensitivity of the particular survey. A question left unanswered by this work is: at what radius does $y$ need to be integrated out to in order to keep from significantly biasing the mass function? Such a study would 
be relatively straightforward if more computationally intensive, although increases in computing power over the last few years makes this less of a concern. Fortuitously, a new, much more extensive set of binary cluster merger simulations is near completion (Chatzikos et al. in prep.), which can be used for this purpose.

\subsection{IC Emission at Hard X-ray Energies}

The remaining Chapters of this thesis have involved efforts to determine or constrain the nonthermal content of clusters by hard X-ray observations of IC emission. Detection of IC emission from clusters with measured radio fluxes from radio halos or relics allows the ICM magnetic field and the total energy in relativistic electrons to be determined. An upper limit on the IC emission from such clusters leads to a upper limit on the energy of relativistic electrons, and a lower limit on the ICM magnetic field.

Nonthermal components of the ICM can affect the use of clusters as cosmological probes. At the most basic level, the state of the ICM relates to the total gravitational potential of a cluster through HSE, which allows cluster masses to be estimated. However, in essentially all cases only the thermal ICM gas is being observed, while HSE must include the pressure contributions from all phases of the ICM.

While diffuse radio synchrotron emission has been clearly observed in $\gtrsim 50$ clusters, detections of the corresponding IC emission have been marginal at best. If IC fluxes are as bright as indicated by these low significance detections, this would imply low values of $B$. Then, the intracluster magnetic fields cannot be dynamically im-

portant in the ICM. But these marginal IC detections need to first be independently confirmed. 


\subsubsection{IC Emission from the Coma Cluster}

In Chapters 3 and 4, we attempt to do just that for the IC emission seen in the Coma cluster. Using hard X-ray instruments onboard the Suzaku and Swift satellites, in conjunction with $X M M-N e w t o n$ measurements of the softer X-ray spectrum to constrain the thermal emission from the cluster, we find no evidence for an IC component in Coma's spectrum, even though we have the sensitivity to detect the signal previously reported. Using the Suzaku HXD-PIN detector and XMM-Newton, we set an upper limit on the nonthermal hard X-ray flux from Coma of $6.0 \times 10^{-12} \mathrm{erg} \mathrm{cm}^{-2} \mathrm{~s}^{-1}$ in the $20-80 \mathrm{keV}$ band.

One difference between our Suzaku HXD-PIN observations and earlier BeppoSAX measurements is that the field of view (FOV) of the HXD-PIN is smaller. The Suzaku HXD-PIN FOV is large enough to cover the full observed extent of the Coma radio halo and nearly all of the thermal X-ray emission from the cluster. However, if the IC emission came from a much larger regions than the observed radio halo, then our Suzaku HXD-PIN observation would miss some of the flux. Thus, a very large extent for the IC emission might reconcile our upper limits with the previous claimed detections with BeppoSAX and RXTE. To test the possibility, we used the Swift BAT survey data to image the Coma cluster at hard X-ray energies, and search for very extended IC emission. No such emission was found, which effectively removes the possibility that Coma has very strong but very extended IC emission.

\subsubsection{IC Emission from the HIFLUGCS Cluster Sample}

In a similar analysis of 58 bright clusters using the Swift data, presented in Chapter 5, we were unable to detect nonthermal emission at a significant level in any of them, and find only marginal evidence cumulatively in the subsample of radio halo 
and/or relic clusters. While perhaps disappointing, the fact that IC emission is still below the sensitivity of current instruments should not be entirely surprising. Direct measurements of the intracluster magnetic field from Faraday RM studies find strengths on the order of several $\mu \mathrm{G}$ Given the radio fluxes of the clusters with radio halo or relics, our hard X-ray sensitivity is sufficient only to detect these clusters if the average magnetic fields are $\lesssim 0.2 \mu \mathrm{G}$.

Unlike most telescopes, the Swift BAT instrument has an enormous FOV - covering roughly one-eighth of the sky in a single pointing - which allows it to accumulate extremely long exposure times everywhere on the sky when operated in survey mode (which is a large fraction of the time). To accomplish this feat, the BAT sacrifices sensitivity due to the high background rate. Even with this complication, the long exposures in the survey result in a good sensitivity. The long exposures give the BAT survey leverage over pointed instruments, such as the Suzaku HXD, which are more sensitive per unit time.

\subsubsection{Future Work on IC Emission}

At present, we believe that the greatest limitation of the BAT survey with regard to galaxy clusters is the flux calibration of the lower energy bands. Especially in the stacked spectra, the first and possibly second energy bands fall systematically below the best-fit model by an amount that cannot be explained by an incorrect crossnormalization or assumed Crab spectrum. Instead, because fluxes are only really valid for sources with spectra similar to the Crab, which serves as the flux calibration source for the survey, the much steeper thermal spectra of clusters are biased low. This effect would be less problematic if the BAT bandpasses were less broad, or the response inside the bands were less variable. Luckily, the BAT survey is not limited to 
8 spectral channels; there are 80 channels native to the instrument. Regenerating the survey in these 80 channels requires no new data or technical capabilities, only more computing time. If the survey data were regenerated in these narrower bandpasses, the problems with calibration and the variation of the response within the broader bands would be essentially eliminated. A re-analysis of the clusters studied here would then be trivial, once this version of the survey exists, and it should be more sensitive to a nonthermal excess at higher energies since the shape of the hard $(\sim 20$ $\mathrm{keV}$ ) thermal emission will be better constrained. The opportunity to carry out this project is especially aided by the fact that I will continue my career at the NASA Goddard Space Flight Center, where the Swift BAT team is located.

Perhaps the most significant difference between this work and other searches for IC emission is how we deal with the thermal component in the hard X-ray spectra. Typically, the temperature of the gas is taken from observations at lower energies, fixed, and then combined with a nonthermal component to see if an excess exists and can be well fit by a power law (e.g., Nevalainen et al. 2004). This method only works, however, if the gas is almost completely isothermal, which is not the case generally and especially for clusters hosting radio halo or relics since they are also undergoing merger events. For even a modestly multi-temperature gas, the temperature you measure will sensitively depend on the bandpass over which the data are fit (Cavagnolo et al. 2008a). By considering only the highest energy (>2 keV) XMM-Newton data and spatially coincident regions, we ensure that the thermal component is accurately constrained so that only true nonthermal excesses are detectable.

Therefore, given the larger amount of high quality data now available at soft $(E<$ $10 \mathrm{keV}$ ) energies, it is worthwhile to re-analyze past data using this methodology. The BeppoSAX, RXTE, and $A S C A$ era resulted in a handful of possible detections of 
nonthermal X-ray emission. In massive systems, the clusters with the most significant nonthermal detections are Coma, A2256, A2319, A2163, and A3376; unclear but potential IC emission was also observed in A754, A3667, A2199, the Bullet cluster (1E 0657-56), and the Centaurus cluster. A hard component was also observed in several lower mass clusters or groups with $A S C A$ : the Fornax cluster, HCG 62, and RGH 80. For all of these clusters and groups, with the exception of RGH 80, long Suzaku observations ( $\gtrsim 100 \mathrm{ks}$ ) are available in the HEASARC archive. However, only about half of the observations have been published so far, and of those, none except for the work on Coma and A3667 have carefully considered the contribution of thermal emission to the HXD spectrum. In the observations of A3376 (Kawano et al. 2009) and A2319 (Sugawara et al. 2009), the temperature of the thermal component was taken from the simultaneous XIS data at softer energies, but a more detailed analysis of the thermal emission, for this purpose, was not done. We can also compare the hard X-ray flux from Suzaku with that detected by Swift, at least for the clearly extended clusters (in the BAT), to further increase sensitivity.

\subsection{Low Frequency Synchrotron Emission}

Another uncertainty in this analysis is the IC spectral index. Radio halos and relics have steep spectra $(\alpha \lesssim-1)$ and thus are expected to be significantly brighter at low frequencies $(<600 \mathrm{MHz})$, making them easier to detect and study; in fact, the next generation of low frequency observatories (LOFAR and LWA) should find an order of magnitude more halos and relics (Cassano et al. 2010). Also, low frequency observations are more appropriate for comparisons with potential IC emission at hard X-ray energies; e.g., for $B \sim 1 \mu \mathrm{G}$, the IC-producing electrons detected at $\gtrsim 20 \mathrm{keV}$ are radiating their synchrotron spectrum primarily at $\nu \gtrsim 70 \mathrm{MHz}$. Data at these 
frequencies are important for another reason: while the spectra from the lower energy IC-producing electrons are expected to be flatter, it is common practice to assume the power law shape measured at higher (typically $1.4 \mathrm{GHz}$ ) frequencies remains similarly steep at low frequencies. This might result in IC detections/limits giving values for $B$ that are biased high. Continuing a focus on the Coma cluster, there is new, very sensitive data at $74 \mathrm{MHz}$ from the VLA and at $235 \mathrm{MHz}$ and $610 \mathrm{MHz}$ from the GMRT of the cluster halo and relic that is waiting to be analyzed. Characterizing the flux and spectrum at these low frequencies will remove uncertainties in the presumed IC spectral model and put more stringent constraints on the intracluster magnetic field strength.

\subsection{Cosmic Rays in the ICM}

While synchrotron and IC emission provide information on $B$ and the relativistic electron population, the only direct constraints on cosmic ray ions come from the $\gamma$-ray regime. Several theories (Brunetti et al. 2007; Colafrancesco \& Marchegiani 2008) that explain the nonthermal emission at either radio and/or X-ray frequencies also predict $\gamma$-rays that should be detectable with the Fermi LAT instrument within the first few years of operation. By the end of next year, the survey sensitivity will probe $\mathrm{GeV}$ cosmic ray densities to a few percent relative to the thermal energy density, comparable to current TeV limits set by H.E.S.S. (Domainko et al. 2009) and MAGIC (Aleksić et al. 2010). The cosmic ray density directly relates to electron densities in models where the electrons result from neutral pion decays after hadronic collisions, and it also allows the electron to ion fraction, which is currently poorly unconstrained, to be measured. The combined measurements at radio, X-ray, and $\gamma$-ray energies will place the strongest constraints yet on the nonthermal component 
of the ICM. An estimate of the cosmic ray population density is especially relevant for IC in terms of the hadronic model for the origin of the emitting electrons, since they are directly related. Even if Fermi observations do not detect cluster $\gamma$-rays, the upper limits should exclude a hadronic origin for the electrons producing radio halos and relics.

\subsection{Future Observatories}

Lastly, it should be pointed out that this work is directly relevant for the next generation of X-ray telescopes, which will focus hard X-ray photons for the first time. Shorter-term missions, such as NuSTAR (Harrison et al. 2005), will be limited in the number of clusters they can observe, and so it is important to identify those clusters with the most significant evidence for nonthermal emission. This is also true for guest observer missions like Astro- $H$, which will require strong proposals in order to get the necessary observing time. For both telescopes, the almost 10-fold increase in effective area at hard energies will finally allow dynamically-important magnetic field strengths to be definitively constrained even with IC upper limits, though the smaller FOV of their detectors will require additional care during target selection. With these missions set to launch over the next several years, the immediate future of this field is bright. 


\section{Appendix A}

\section{Fitting Simulation Data}

We use the same basic forms and procedures to fit the merger boosts discussed in RSR, Appendix B. For the integrated Comptonization parameter, the boosted part of the cumulative time distribution histograms is well-fit by hyperbolas similar in form to equation (B1) of RSR with a slight modification:

$$
\ln \left(\frac{t}{t_{\mathrm{sc}}}\right)=\sqrt{\left(\left\{\frac{Y}{Y(0)}-\left[\frac{Y}{Y(0)}\right]_{\mathrm{peak}}-1\right\}^{2}-1\right)\left(\epsilon^{2}-1\right)}-\ln \left(\frac{t}{t_{\mathrm{sc}}}\right)_{Y} .
$$

Three parameters describe the function: the maximum boost $[Y / Y(0)]_{\text {peak }}$, the boost duration $\left(t / t_{\mathrm{sc}}\right)_{Y}$, and the eccentricity of the hyperbola $\epsilon$. The fit values for these parameters between simulation runs could be reproduced with the same functions of fractional mass increase $f_{M}$ and normalized impact parameter $b^{\prime}$ used in RSR, provided here for completeness:

$$
\left[\frac{Y}{Y(0)}\right]_{\text {peak }}\left(f_{M}, b^{\prime}\right)=\frac{A f_{M}^{B}}{C+b^{\prime 2}}+1
$$




$$
\begin{gathered}
\epsilon\left(f_{M}, b^{\prime}\right)=\left(\frac{A f_{M}^{B}}{C+b^{\prime 2}}\right) \\
\ln \left(\frac{t}{t_{\mathrm{sc}}}\right)_{Y}=G \frac{\ln \left(M_{<}+M_{>}\right)-H \ln \left(M_{<}^{1 / 3}+M_{>}^{1 / 3}\right)}{I+b^{\prime 2}} .
\end{gathered}
$$

As in the text, the impact parameter is scaled by the core radii of the two merging clusters, $b^{\prime}=b /\left(r_{c<}+r_{c>}\right), M_{<}$and $M_{>}$are the masses of the less massive and more massive cluster (in $M_{\odot}$ ), respectively, and the fractional mass increase $f_{M} \equiv$ $M_{<} /\left(M_{<}+M_{>}\right)$. Motivations for these forms are given in Appendix B of RSR.

The variation of $y_{\max }$ with the viewing angle of the merger causes the histograms of values of time versus $y_{\max }$ to be broader than the histograms for $Y$ (Figure 2.3). This difference makes hyperbolae a poor representation of the histogram shapes. We find a suitable replacement in another 3 parameter function

$$
\ln \left(\frac{t}{t_{\mathrm{sc}}}\right)=P \ln \left(1-\frac{y_{\mathrm{max}}}{y_{\mathrm{peak}}}\right)-\frac{1}{2} \frac{y_{\mathrm{max}}}{y_{\mathrm{peak}}}-\ln \left(\frac{t}{t_{\mathrm{sc}}}\right)_{y}
$$

with similarly defined parameters for the maximum $y_{\max }$ boost $\frac{y_{\text {peak }}}{y_{\max }(0)}$, the power law slope $P$, and the boost duration $\ln \left(\frac{t}{t_{\mathrm{sc}}}\right)_{y}$ :

$$
\begin{gathered}
\frac{y_{\text {peak }}}{y_{\text {max }}(0)}=\frac{A f_{M}^{B}}{C+b^{\prime 1.3}}+1, \\
P=\left(\frac{D}{F+b^{\prime 1.5}}\right)^{-1}, \\
\ln \left(\frac{t}{t_{\mathrm{sc}}}\right)_{y}=G \frac{\ln \left(M_{<}+M_{>}\right)-H \ln \left(M_{<}^{1 / 3}+M_{>}^{1 / 3}\right)}{I+b^{\prime 2}} .
\end{gathered}
$$

The best-fit values found for $A-I$ are given in Table A.1. Note that $A-I$ are found assuming that the value of $y_{\max }(0)$ is taken along the merger axis, which is twice the value of $y_{\max }(0)$ used in Figures 2.2 and 2.3, for which the value perpendicular to the 
merger axis is used.

Table A.1. Fitting Parameters for Merger Boost Histograms

\begin{tabular}{lccccccccc}
\hline \hline Boost & $\mathrm{A}$ & $\mathrm{B}$ & $\mathrm{C}$ & $\mathrm{D}$ & $\mathrm{E}$ & $\mathrm{F}$ & $\mathrm{G}$ & $\mathrm{H}$ & $\mathrm{I}$ \\
\hline$Y / Y(0)$ & 95.69 & 0.8793 & 66.72 & 94.83 & 0.3621 & 173.3 & 33.36 & 0.2793 & 473.3 \\
$y_{\max } / y_{\max }(0)$ & 26.55 & 0.5776 & 4.052 & 6.310 & - & 4.569 & 2.250 & 1.785 & 13.76 \\
\hline
\end{tabular}




\section{Appendix B}

\section{XMM-Newton EPIC-pn-Swift BAT \\ Cross-Calibration}

Because the systematic uncertainties in the survey-averaged spectrum of the Crab Nebula are smaller than the uncertainties in the BAT survey response matrix, BAT survey fluxes are tied to the Crab fluxes in each band since the systematic uncertainties in the survey-averaged spectrum of the Crab Nebula are smaller than the uncertainties in the BAT survey response matrix (see Tueller et al. 2010, Sec. 4.5). This method also requires that the intrinsic Crab spectrum be defined since its exact spectrum remains somewhat uncertain (see Sec. B.2 below), particularly at higher X-ray energies. In practice, though, we are less concerned with an accurate absolute calibration for the BAT than we are with, in this case, an accurate calibration

relative to our XMM-Newton EPIC-pn spectrum. Therefore, instead of prescribing a canonical Crab spectrum as close to the true spectrum as it has been measured thus far, we need to set it to the Crab spectrum as measured by the XMM-Newton EPIC-pn instrument over the energy range we consider. Otherwise, systematic calibration errors between the instruments could significantly affect our result, since our 
goal is to detect excess radiation at hard energies due to nonthermal emission. Errors leading to steeper (flatter) XMM-Newton spectra and flatter (steeper) Swift spectra, for example, will reduce (increase) the thermal contribution at higher energies and similarly enhance (suppress) a nonthermal signal. In other words, any systematic miss-calibrations are mimicked in the BAT calibration so that thermal and nonthermal models can be simply applied during joint fits of the data.

\section{B.1 The Spectrum of the Crab According to XMM- Newton}

Because of its high X-ray flux, simple spectrum, and lack of significant variability, the pulsar wind nebula of the Crab supernova remnant has been proposed as an X-ray standard flux calibrator (Kirsch et al. 2005). Observations over a large range of energies and with many diverse instruments reveal an intrinsic spectrum nearly consistent with a single power law; however, the photon index and normalization determined by each detector exhibit small but not insignificant differences (Kirsch et al. 2005; Weisskopf et al. 2010). XMM-Newton EPIC-pn measurements, which require that observations are made in burst mode due to XMM-Newton's large collecting area, are best fit with a steeper than average photon index $(\Gamma=2.13$ versus $\langle\Gamma\rangle=2.08$ for simultaneous fits to many instrument observations, Kirsch et al. 2005) that is driven by the shape of the spectrum from $0.5-2 \mathrm{keV}$. However, the residuals to this fit in their Figure 7 suggest that for energies above $2 \mathrm{keV}$, the XMM-Newton photon index is more in line with the average, and since we only consider energies above $2.3 \mathrm{keV}$, we need to determine what the Crab spectrum is measured to be in this range.

Instead of fitting the Crab spectrum directly, we choose to compare the XMM- 
Newton data to Suzaku XIS0 data, which has been well calibrated and consistently fit over its energy range using observations of the Crab. Spectra from each instrument are extracted from identical spatial regions at the center of the Coma cluster (specifically, Region 10 from Wik et al. 2009), where both the gas temperature and surface brightness are roughly constant. Fitting each spectrum from $2.3-12 \mathrm{keV}$ with a single temperature APEC model, we find the $X M M$-Newton-derived temperature to be slightly, though not insignificantly, lower than the Suzaku temperature: $8.32 \mathrm{keV}$ versus $8.90 \mathrm{keV}$. The lower EPIC-pn temperature is consistent with an increasingly larger effective area at higher energies relative to Suzaku's high energy effective area; positing that $X M M-N e w t o n ' s$ calibration is correct, the relative Suzaku effective area at larger energies should be increased as a function of energy, which would lower the flux and therefore the temperature. We model this effect as multiplicative power law component to the Suzaku APEC fit, which simultaneously accounts for both the gradient and the overall cross-normalization between the two instruments. Fixing the APEC model parameters to those found with the fit to the $X M M-N e w t o n$ spectrum, we find this modification to the Suzaku calibration: $f\left(A_{e f f}\right)=0.923(E / 1 \mathrm{keV})^{0.045}$. In other words, Suzaku spectra are flatter than XMM-Newton spectra and have similar hard band fluxes; however, note that the XMM-Newton effective area had previously been reduced by $15 \%$, as per the analysis in Wik et al. (2009), in order to match the 2-10 keV EPIC-pn and XIS0 fluxes. Dividing the XIS0 best-fit Crab spectrum of Ishida et al. $\left[F_{\text {Crab,XIS0 }}(E)=9.51(E / 1 \mathrm{keV})^{-2.05}\right.$, given in Suzaku Memo $\left.2007-11^{1}\right]$ by $f\left(A_{e f f}\right)$ finally yields the correct parameters for the $X M M$-Newton fit to the Crab spectrum in the energy range of interest:

$$
F_{\text {Crab }}(E)=10.30\left(\frac{E}{1 \mathrm{keV}}\right)^{-2.095} \text { photons } / \mathrm{cm}^{2} / \mathrm{s} .
$$

\footnotetext{
${ }^{1}$ http://www.astro.isas.ac.jp/suzaku/doc/suzakumemo/suzakumemo-2008-03.pdf
} 
We take this equation as our canonical Crab spectrum. Then, the true flux of a source in each of the 8 bands is given by the BAT source count rate in that band divided by the observed Crab BAT count rate in that band, and multiplied by the spectrum in Equation (B.1) integrated over the band (see Tueller et al. 2010, eqns. 2-4). This conversion factor is reported in Table B.1 as the "Flux Calib.".

While this method is the standard way to create spectra from survey data, it is technically only valid for source spectra that have a shape similar to the Crab. Unfortunately, the survey redistribution matrix, which could properly account for arbitrary spectral shapes, is more uncertain than the observed Crab fluxes (Tueller et al. 2010). Thermal emission above $\sim 10 \mathrm{keV}$ is typically much steeper than the spectral index in Equation (B.1), so we would prefer to include an approximate redistribution matrix that will handle other such spectral models correctly. To do this, we take a standard response function for an on-axis source from a single observation and multiply the input energies by a smooth function so that the flux-converted Crab spectrum matches Equation (B.1) to $<1 \%$ in all energy bands. The addition of this redistribution matrix has a minor effect on spectral fits generally, but it does improve the quality of fits using a thermal model, so we employ it throughout.

\section{B.2 The Hard X-ray Spectrum of the Crab}

With this approach, accurate conversions from BAT count rates to true fluxes are not guaranteed. The goal instead is to match the BAT calibration with the EPIC-pn calibration, which ensures that spectral models can be applied seamlessly between the XMM-Newton and Swift spectra in joint fits. While fluxes quoted hereafter may differ from their true fluxes, the relative amounts of thermal versus nonthermal emission in the joint spectra - considering both their cross-normalization factor and shape - 
Table B.1. Swift BAT Error Factors

\begin{tabular}{|c|c|c|c|c|c|c|c|c|c|c|c|c|c|}
\hline Band & $\begin{array}{l}\text { Energies } \\
(\mathrm{keV})\end{array}$ & $\begin{array}{l}\text { Flux Calib. }^{\mathrm{a}} \\
\quad\left(\mathrm{cm}^{-2}\right)\end{array}$ & $\sigma_{\text {bgd }}{ }^{b}$ & $\begin{array}{c}\mathrm{PtSrc} \\
f_{m}\end{array}$ & $\begin{array}{l}\text { The } \\
N_{\mathrm{PSF}}\end{array}$ & $\begin{array}{l}\text { mal } \\
f_{m}\end{array}$ & $\mathrm{R} 25$ & R30 & R35 & $\begin{array}{r}\mathrm{R} 40 \\
f_{m}\end{array}$ & $\mathrm{R} 45$ & R60 & KW \\
\hline E1 & $14-20$ & 16.55 & 2.37 & 1.44 & 2.09 & 1.80 & 1.77 & 1.60 & 1.73 & 1.98 & 2.08 & 2.06 & 2.22 \\
\hline $\mathrm{E} 2$ & $20-24$ & 11.28 & 1.05 & 1.32 & 2.02 & 1.75 & 1.39 & 1.53 & 1.56 & 1.22 & 1.60 & 1.57 & 1.94 \\
\hline E3 & $24-35$ & 10.89 & 1.63 & 1.35 & 1.97 & 1.59 & 1.41 & 1.70 & 1.82 & 1.51 & 1.81 & 1.91 & 2.08 \\
\hline E4 & $35-50$ & 10.46 & 1.12 & 1.31 & 1.90 & 1.66 & 1.40 & 1.59 & 1.53 & 1.18 & 1.62 & 1.64 & 2.15 \\
\hline E5 & $50-75$ & 9.75 & 1.02 & 1.30 & 1.88 & 1.27 & 1.59 & 1.35 & 1.54 & 1.28 & 1.45 & 1.41 & 2.04 \\
\hline E6 & $75-100$ & 13.00 & 0.99 & 1.29 & 2.03 & 1.34 & 1.37 & 1.52 & 1.25 & 1.36 & 1.51 & 1.57 & 1.85 \\
\hline E7 & $100-150$ & 24.48 & 1.75 & 1.31 & 2.67 & 1.40 & 1.47 & 1.59 & 1.57 & 1.31 & 1.52 & 1.48 & 1.72 \\
\hline E8 & $150-195$ & 75.19 & 3.84 & 1.31 & 1.24 & 1.42 & 1.45 & 1.26 & 1.29 & 1.23 & 1.65 & 1.56 & 1.93 \\
\hline & fuse $N_{\text {PSF }}$ & ndep. of $E$ & & 1.00 & & & 4.56 & 6.60 & 8.98 & 11.81 & 14.94 & 26.42 & 41.03 \\
\hline
\end{tabular}

a "Flux Calibration," defined such that the incidence photon flux at the Earth (photons $\mathrm{cm}^{-2} \mathrm{~s}^{-1}$ ) is given by the BAT source count rate multiplied by Flux Calib.

${ }^{\mathrm{b}}$ Units are $10^{-5}$ photons $\mathrm{s}^{-1} \mathrm{~cm}^{-2}$ 
are carefully conserved. Ultimately, because our BAT calibration method relies on using the Crab as a flux standard, and since the true Crab spectrum is not known, the choice of a canonical Crab spectrum is at some level arbitrary.

Even so, the hard band fluxes derived herein should be consistent with fluxes derived from other missions. Using the same power law form as for Equation (B.1), Kirsch et al. (2005) found a range of normalizations and photon indices for several instruments that overlap with the BAT energy bands: Beppo-SAX PDS: $8.84 E^{-2.126}$; RXTE PCA: $11.02 E^{-2.120}$; RXTE HEXTE: $9.9 E^{-2.090}$; INTEGRAL ISGRI: $15.47 E^{-2.252}$; and INTEGRAL SPI: $15.9 E^{-2.203}$. Also, the Suzaku PIN fit of $10.93 E^{-2.090}$ is consistent with both the scatter in the above results and our adopted spectrum. Ignoring the photon indices derived from $X M M$-Newton data, there seems to be a steepening in the Crab spectrum at higher energies, which means that our relatively flat photon index may over-predict harder band fluxes and thus enhance a potential nonthermal signal. Our Crab spectrum also has a slightly higher overall flux (in the $20-80 \mathrm{keV}$ band), so that fluxes and upper limits may be biased high, though any such biasing would be well within the absolute calibration uncertainties of all the above missions. 


\section{Appendix C}

\section{Extracting BAT Fluxes from Extended Sources}

Very extended, diffuse emission is difficult to detect with coded mask instruments, since the shadow pattern of the mask on the detectors gets smeared out and the signal becomes indiscernible from the background. However, small scale extended emission is detectable, as long as its size is less than the minimum scale necessary to dilute the distinguishability of the mask pattern. In the following, we show that, by simulating extended sources as collections of point sources, this minimum scale is larger than our region of interest and that essentially $100 \%$ of the diffuse emission can be detected.

\section{C.1 BAT Point Spread Function}

For on-axis sources in the BAT FOV, the point spread function (PSF) has a full width half maximum (FWHM) of $\sim 22^{\prime}$. Because the sky image is basically the crosscorrelation function of the coded mask with the count rates in the individual detectors, it does not represent the intensity per solid angle (i.e. within a pixel). Instead, a 
pixel value is proportional to the flux of a point source at that location. The width of the PSF is actually due to oversampling the sky plane, not the scattering of photons inside the instrument, and it depends on the size of individual mask element shadows on the detector relative to the size of detector pixels.

As such, the PSF should not be summed in order to derive the source flux - this is provided by the central peak value - and its FWHM depends on the off-axis angle of the source. The distance between the mask and detector increases as the off-axis angle increases, so an angular separation at large off-axis angles produces a more dramatic shift in the shadow pattern across the detector pixels than more on-axis positions, which effectively reduces the oversampling factor and leads to narrower FWHM. Since survey images are created from many "random" individual pointings, each with a given source located at a different off-axis angle, the survey PSF will have an average FWHM and uniform shape, which is roughly Gaussian. Simple Gaussian fits to all the $\gtrsim 10 \sigma$ sources in all 8 bands yield an average FWHM of 19!47, irrespective of $\mathrm{S} / \mathrm{N}$ or energy band, essentially identical to the value determined for the 22-month survey (Tueller et al. 2010).

The PSF shape is described by a Gaussian to first order, which is not surprising given the non-repeating, randomly filled mask and the many pointings that contribute to the flux at each position. However, as is clear from the residuals to a Gaussian fit to the 14-20 keV band Crab data and another source (Cyg X-2) in Figure C.1, deviations on the order of $1 \%$ of the flux exist (and are significantly larger than the root-mean square (RMS) of the background in this case). While this deviation does not strongly impact the flux of point sources, since only the maximum, central value maps to the flux, a diffuse source is composed of overlapping PSFs, where differences in the wings could affect the overall flux. The residual structure in the wings of the 
PSF is mainly eliminated by the addition of the two-part function:

$$
f(r)=p_{0}\left[e^{-r^{2} / 2 \sigma_{\mathrm{PSF}}^{2}}+\frac{1}{120}\left\{\begin{array}{ll}
\cos \pi x & x<3 \\
-e^{-\pi(x-3) / 1.19} & x>3
\end{array}\right]+p_{1},\right.
$$

where $r$ is the distance from the center in arcminutes, $p_{0}$ and $p_{1}$ are fit parameters (the normalization and background, respectively), $x=2 r /(1.19 \mathrm{FWHM})+1(x$ has units of radians), and $\sigma_{\mathrm{PSF}}=\mathrm{FWHM} /(2 \sqrt{2 \ln 2})$. The improved fit for the Crab is illustrated in the very bottom left panel of Figure C.1; while in this case the fit is still not perfect, for other sources the fit is typically better (bottom right panel of Figure C.1). We take $p_{0}$ to be our measurement of the flux. While the maximum of the PSF may not exactly correspond to $p_{0}$, since all fluxes are determined this way and are also related to the Crab fluxes, any such bias will cancel out during the conversion from BAT count rates to fluxes. Note that the most the additional terms to $f(r)$ could affect a flux, assuming they, for some reason, poorly represented the true PSF shape, is at the $\lesssim 1 \%$ level.

\section{C.2 Tests of the Detection of Extended Sources}

As discussed in Section 4.3, we extract fluxes for extended sources by fitting a priori model distributions, as opposed to using a method like the "CLEAN" algorithm (Högbom 1974), which reconstructs fluxes from an unknown underlining distribution assuming the PSF shape only. The "CLEAN" method requires some fine-tuning, such as the region of extraction (for clearly detected sources, expanding the region-ofinterest even a little beyond the wings of the source can significantly bias the derived flux), "loop gain," and completion threshold. In our case, since there are only a few 


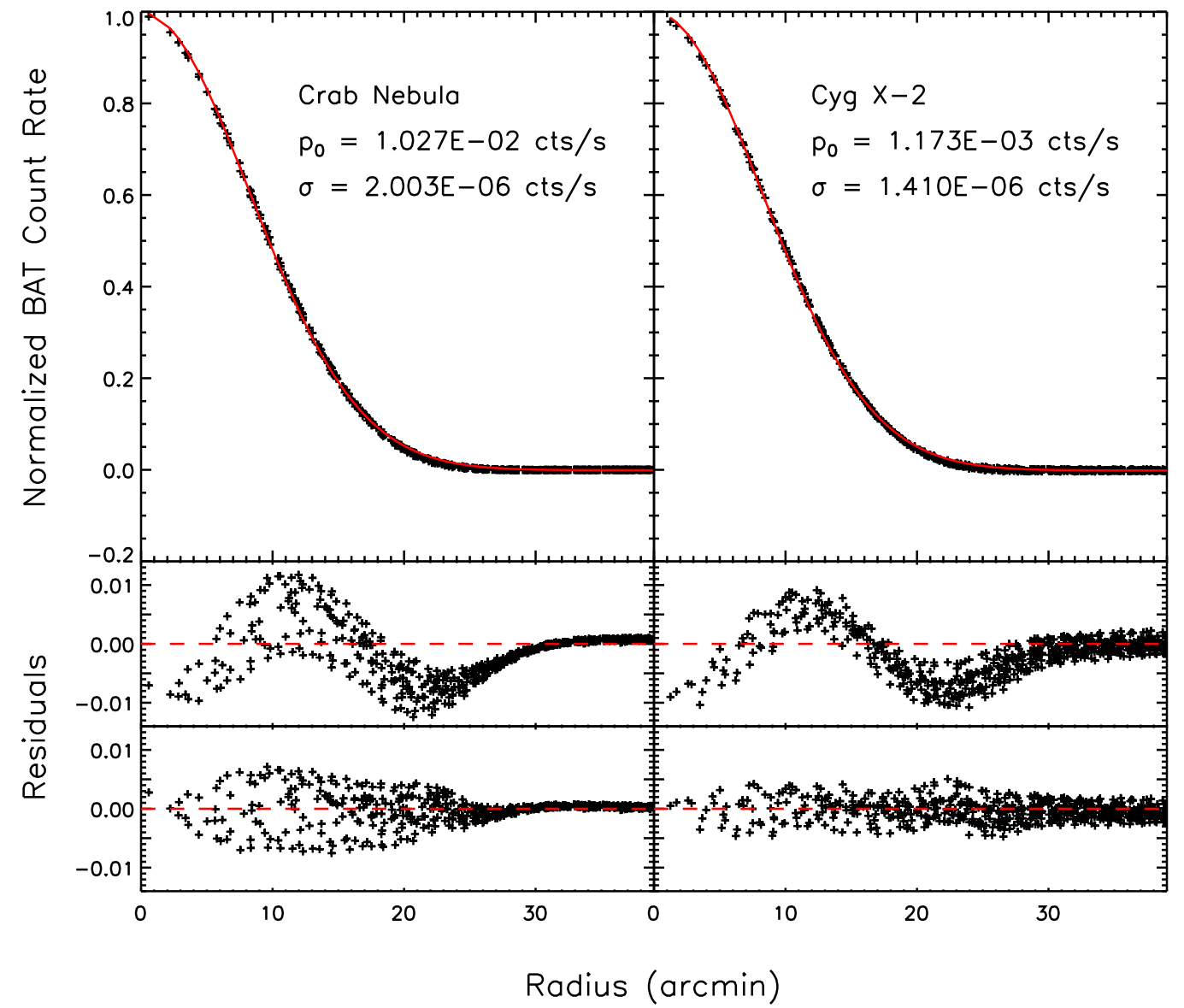

Fig. C.1. - Fits to the BAT PSF. In the top and middle panels, the profiles of two point sources, the Crab and Cyg X-2, are fit to a Gaussian. Large, regular residuals (middle panels) remain, which are mostly removed (at the $<1 \%$ level, bottom panels) after modifying our expression for the PSF (Eqn. C.1). Lingering residuals, which are particularly significant for the Crab but only slightly noticeable for the other source, primarily result from spatial asymmetries due to the actual angular extent of the source, as in the case of the Crab, and the rectangular shape of the BAT instrument, which will cause off-axis sources to be more "squished" in one direction than another. In the latter case, the effect of summing many individual pointings with the detector in various orientations almost, but not entirely, removes this azimuthal component of the PSF shape. 
likely spatial distributions for the thermal and any potential nonthermal emission, we are less likely to produce biased fluxes by first assuming a spatial distribution than by using a method like "CLEAN." We represent a diffuse source as a collection of point sources, each of which is convolved by the PSF (Eqn. C.1) and summed together.

We now test whether diffuse sources are detectable over our scales of interest can be evaluated. In general, we treat extended emission as a collection of closely-spaced point sources, since existing software is built with these sources in mind. Point sources at any position in the BAT FOV are straightforward to simulate with the HEASOFT Swift task batmaskwtimg with the following options set: coord_type=tanxy; distance=1e7; corrections=forward, unbalanced,flatfield; and rebalance=no. This task outputs the fraction of each detector pixel which is illuminated by the source at its input position; a value of 0.45 means that $55 \%$ of the detector area is shadowed by the mask. At this stage, the detector image can be multiplied by the counts or count rate of the source, and several such detector images representing different sources in the FOV can be added together along with a background - all including Poisson statistics. The background can then be fit and subtracted with the task batclean, and finally a reconstructed sky image can be produced via the task batfftimage. For now, to isolate the detectability of diffuse emission by the BAT, we simply add uniformly bright, perfectly known point source masks without background or source Poisson noise, to create circular, extended disks of various radii $R$. Images of the sky are constructed with batfftimage for each disk detector image, and the "observed" disk surface brightness profile is fit for as a function of radius. While even large disks $\left(R \gtrsim 10^{\circ}\right)$ are visibly noticeable in the sky images, large systematic effects induced by the large spatial extent of the emission lead to large RMS noise that eventually destroys its detectability. The recovered surface brightness, relative 
to the input level, of simulated diffuse disks of radius $R$ are presented in Figure C.2. Error bars represent the simple error of the mean $\left(\sigma_{\mathrm{RMS}} / \sqrt{N}\right.$, where $N$ is the number of pixels used to determine $\sigma_{\mathrm{RMS}}$ ), and the smooth as opposed to random variation around the input surface brightness results from their systematic nature. For sources in the size range of interest to us, $R<1.5$, the intrinsic uncertainty due to the telescope design is $\lesssim 3 \%$. As the disk radius increases, the reconstructed surface brightness becomes less and less robust as there are effectively more sources (other parts of the disk) contributing systematic noise to a given location. Note, however, that all of the input source flux is recovered. 


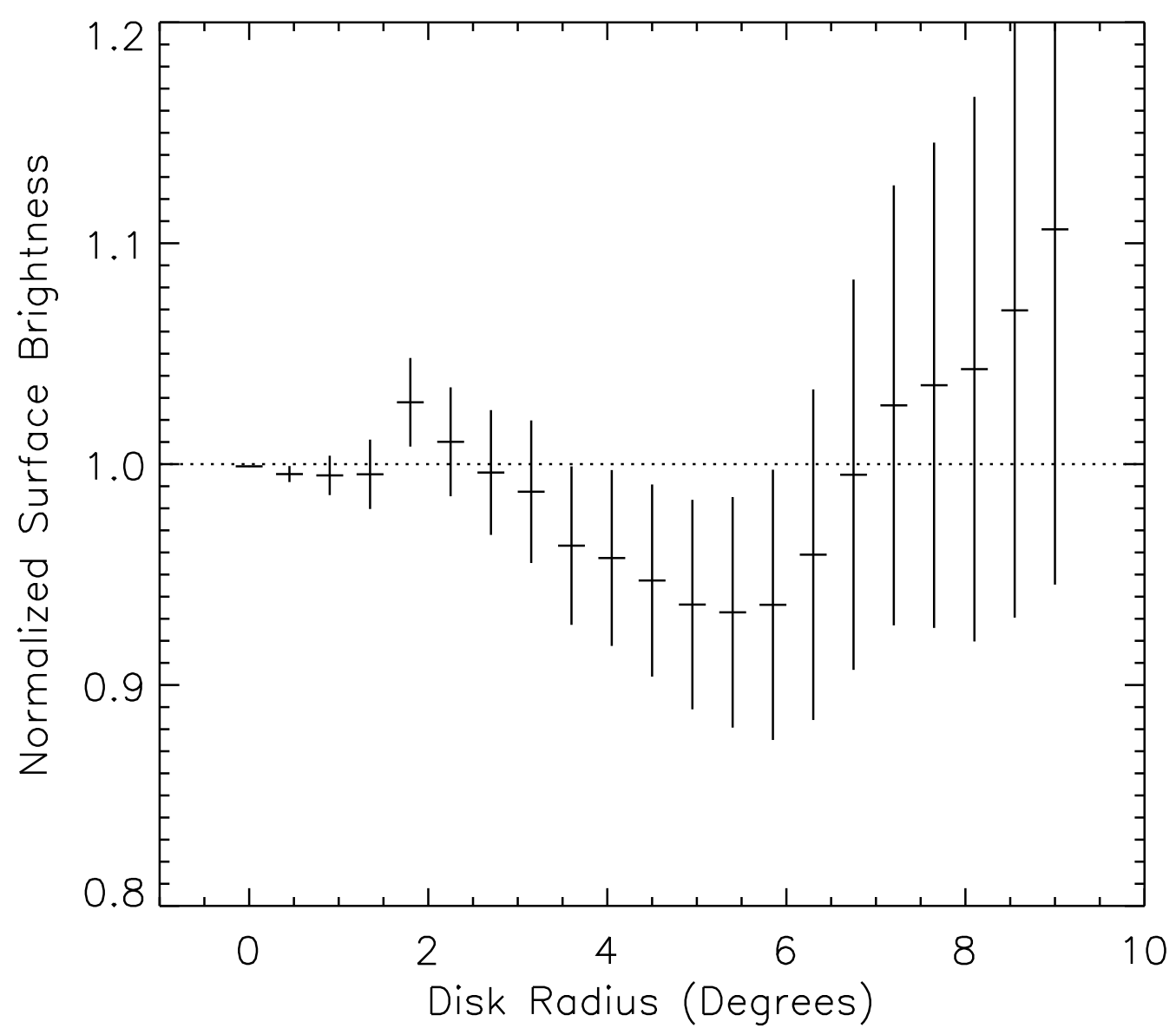

Fig. C.2.- Recovered surface brightness for simulated emission from a uniform surface brightness disk of a given radius. Error bars indicate the statistical error of the mean on the disk surface brightness, though the spatial fluctuations in the sky reconstruction behind this error are due entirely to systematic effects; the "noise" at any position in these simulations is determined by the flux of all the other sources within the FOV, or in this case the other parts of the disk. The variation with radius is smooth instead of random due to the systematic origin of the fluctuations. For angular sizes of interest here, $<90^{\prime}$, the intrinsic uncertainty in the recovered flux of an extended source, due to coded mask imaging techniques, is at most a few percent (based on the size of the error bars). Also, there is no loss in sensitivity to diffuse emission; all of the input flux is recovered, albeit with less and less precision for larger sources. 


\section{Appendix D}

\section{Uncertainties in BAT Fluxes}

\section{D.1 Flux Uncertainties for Point Sources}

The uncertainty in a given flux measurement is encoded in the RMS fluctuations in the local background (Tueller et al. 2010). These fluctuations represent both the statistical fluctuations from shot noise (dominated by the high background rate) and systematic error contributions from the sky reconstruction process. Due to the large number of individual pointings at nearly random positions, most systematic effects nearly average out and lead to a symmetric, nearly Gaussian distribution for blank sky regions. We calculate the RMS of the background $\left(\sigma_{\mathrm{bgd}}\right)$ around Coma in an annulus of radius $15<r<100$ pixels $\left(42^{\prime}<r<4.67\right)$, as is typically done for sources in the BAT survey. The values of $\sigma_{\text {bgd }}$ for each band are given in Table B.1. While this annulus partially includes the region within which we are searching for a diffuse nonthermal signal, the lack of any obvious emission indicates that the derived errors could not be significantly biased. To ensure $\sigma_{\text {bgd }}$ is not biased by low level extended flux, we recalculated it inside an annulus of equal area with an inner radius of $90^{\prime}$ and found a nearly identical value of $\sigma_{\mathrm{bgd}}$ in all 8 bands. 


\section{D.2 Flux Uncertainties for Extended Sources}

The error for a point source, or more correctly the error in the value of a given pixel, presented in Section D.1 does not directly apply to extended sources. Also, we cannot take the standard error from spatial $\chi^{2}$ fits, using the point source error as the error for the flux in each pixel, because nearby pixels are correlated. Helpfully, the expected error for diffuse sources has already been derived by Renaud et al. (2006b) for the IBIS coded mask instrument onboard INTEGRAL. In their appendices, they derive source fluxes and errors in reconstructed sky images from detector images and find the straightforward result that the error in a measurement of an extended source flux is proportional to its spatial area normalized by the area of the PSF function (Renaud et al. 2006b, Eqn. B3). Specifically,

$$
\sigma_{\text {ext }}=\sigma_{\text {bgd }} \sqrt{N_{\mathrm{PSF}}}
$$

where $N_{\mathrm{PSF}}$ is the area of the source normalized by the PSF area. If $I_{X}(\Omega)$ is the surface brightness of the source convolved with the PSF, $I_{X}^{\max }$ is its maximum value, $\Omega$ is the solid angle, and $f(\Omega)$ is the PSF, then

$$
N_{\mathrm{PSF}} \equiv \frac{\int I_{X}(\Omega) d \Omega}{I_{X}^{\max } \int f(\Omega) d \Omega} .
$$

Before we generally apply Equation (D.1) to Swift BAT data, we test whether this prescription does in fact apply to extended sources in the Swift BAT. For each of the diffuse thermal (Section 4.3.1) or nonthermal (Section 4.3.1) spatial models described above, we created 121 simulations of the extended source and a number of other point sources in the FOV, each with detector counts for the source fluxes and background randomly assigned (taken from a Poisson distribution). Each simulated 
observation is made from a unique position on an $11 \times 11$ grid, with the relative positions of all the sources kept intact. Both the variation of position relative to the telescope axis and the inclusion of point sources are necessary to fully recreate the systematic contribution to the error. The simulated detector images are then background subtracted and converted into sky images via the procedure outlined in Section C.2. The total flux of the diffuse sources is chosen so that the signalto-noise ratio is $\sim 20-50$. To check Equation (D.1), fluxes of all the sources are measured in each simulated sky image, and the average standard deviation of the point source fluxes are compared to the standard deviation of the diffuse source flux. We find that the estimated errors for the diffuse models (Thermal band E1, Disks R25-R60, and KW) generally fall below the expected trend with $N_{\mathrm{PSF}}$ in Figure D.1. This discrepancy may be due to the number of simulations we were computationally limited to performing - the distribution of fluxes is only roughly Gaussian - or it may represent a true deviation from the results of Renaud et al. (2006b). However, to be safe we use Equation (D.3) to calculate the error of fluxes extracted with the corresponding model.

There is one additional modification to errors on fluxes extracted with our methodology. Because we fit a spatial model, convolved by the PSF, to the BAT image data, the error in the flux is not just the standard deviation of nearby background pixels, but it depends on how the model is fit to all the pixels. For example, the distribution of normalizations from many fits of a Gaussian function to random data of mean zero and standard deviation $\sigma_{\text {bgd }}$ will not equal $\sigma_{\text {bgd }}$ but some value $<\sigma_{\text {bgd }}$ depending on the pixel scale. A delta function, or Gaussian of width zero, will produce a distribution consistent with $\sigma_{\text {bgd }}$, since this is identical to measuring the standard deviation, but anything wider finds an average over several pixels, and therefore the distribution 


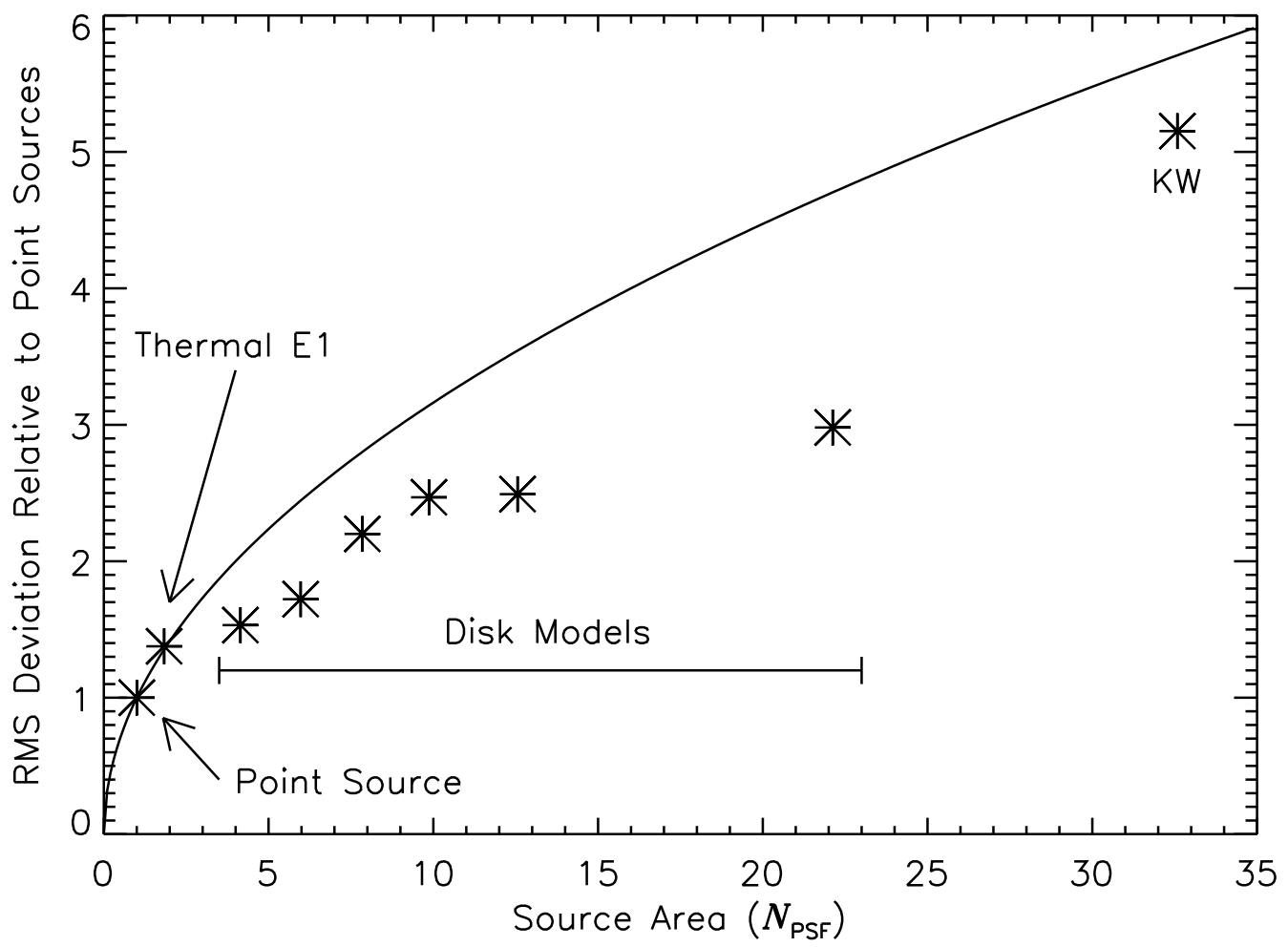

Fig. D.1. - The standard deviation of the best-fit normalization for 100 simulations of each spatial model considered in this work. Both photon noise (in the background and source flux) and systematic effects (the influence of other point sources in the FOV and the relative off-axis angle of all sources relative to the detector) are included. The model area is shown in terms of the equivalent number of PSF areas, $N_{\mathrm{PSF}}$. For the thermal spatial models, only the lowest energy band (E1: 14-20 keV) is plotted for clarity. The solid line represents the expected $\sqrt{N_{\mathrm{PSF}}}$ dependence of the error (Equation D.1). The simulated values of the errors for the extended models fall below this result, and possible explanations of this behavior are briefly discussed in the text. Note that for the thermal E1 model, the difference in the value of $N_{\text {PSF }}$ shown here and in Table B.1 results from the difference between the survey PSF and the on-axis PSF used in these simulations. 
of normalizations will tend to be closer to the mean of the random pixels. For our purpose, where the normalization is related to the source flux, the correct error of a flux should come from the distribution of model fits to background (empty) regions of the survey, which may not be equivalent to $\sigma_{\mathrm{bgd}}$. Unlike in the above example, neighboring pixels in the survey are correlated due to oversampling - this is essentially the origin of the PSF - and so the standard deviation of model normalizations will be affected by this correlation. Generally, the distribution of normalizations will be larger than $\sigma_{\mathrm{bgd}}$ in this case, as $\chi^{2}$ minimization will be more influenced by the larger fluctuations near the flux extraction region. The net effect does not significantly change the error distribution shape, but simply inflates the effective standard deviation by some factor, $f_{m}$, which is both model-dependent (varying from $1.4 \sigma_{\mathrm{bgd}}$ for a point source to $2.24 \sigma_{\mathrm{bgd}}$ for the $\mathrm{KW}$ model) and energy dependent since the noise properties vary slightly from band-to-band. The total flux uncertainty for a diffuse source in the BAT survey is adjusted from Equation (D.1) to become

$$
\sigma_{\text {diffuse }}=f_{m} \sigma_{\text {ext }}=f_{m} \sigma_{\text {bgd }} \sqrt{N_{\mathrm{PSF}}}
$$

The precise value of $f_{m}$ is determined from the standard deviation of fits to 100 blank sky regions, in which we avoid obvious $(>5 \sigma)$ sources and the Galactic plane $\left(b>20^{\circ}\right)$. These factors are reported for each band in Table B.1. Not including this error contribution results in spectral fits with unacceptably high $\chi^{2}$ values. 


\section{References}

Abell, G. O. 1958, ApJS, 3, 211

Abell, G. O., Corwin, Jr., H. G., \& Olowin, R. P. 1989, ApJS, 70, 1

Ackermann, M., et al. 2010, ApJ, 717, L71

Adami, C., Biviano, A., Durret, F., \& Mazure, A. 2005, A\&A, 443, 17

Aharonian, F., et al. 2009a, A\&A, 495, 27

-. 2009b, A\&A, 502, 437

Ajello, M., et al. 2008, ApJ, 689, 666

—. 2009, ApJ, 690, 367

Aleksić, J., et al. 2010, ApJ, 710, 634

Arnaud, M., et al. 2001, A\&A, 365, L67

Bahcall, N. A., \& Fan, X. 1998, ApJ, 504, 1

Barcons, X., Mateos, S., \& Ceballos, M. T. 2000, MNRAS, 316, L13

Bartlett, J. G. 2006, ArXiv Astrophysics e-prints

Bazzano, A., et al. 1990, ApJ, 362, L51 
Begelman, M. C., Rees, M. J., \& Blandford, R. D. 1979, Nature, 279, 770

Bertschinger, E. 1985, ApJS, 58, 39

Birkinshaw, M. 1999, Phys. Rep., 310, 97

Boldt, E. 1987, in IAU Symposium, Vol. 124, Observational Cosmology, ed. A. Hewitt, G. Burbidge, \& L. Z. Fang, 611-615

Bonafede, A., Feretti, L., Murgia, M., Govoni, F., Giovannini, G., Dallacasa, D., Dolag, K., \& Taylor, G. B. 2010, A\&A, 513, A30+

Bonaldi, A., Tormen, G., Dolag, K., \& Moscardini, L. 2007, ArXiv e-prints, 704

Bonamente, M., Lieu, R., \& Bulbul, E. 2009, ApJ, 696, 1886

Bonamente, M., Nevalainen, J., \& Lieu, R. 2007, ApJ, 668, 796

Bond, J. R., Cole, S., Efstathiou, G., \& Kaiser, N. 1991, ApJ, 379, 440

Bradt, H., Mayer, W., Naranan, S., Rappaport, S., \& Spada, G. 1967, ApJ, 150, $\mathrm{L} 199+$

Briel, U. G., et al. 2001, A\&A, 365, L60

Brunetti, G., \& Blasi, P. 2005, MNRAS, 363, 1173

Brunetti, G., Venturi, T., Dallacasa, D., Cassano, R., Dolag, K., Giacintucci, S., \& Setti, G. 2007, ApJ, 670, L5

Bryan, G. L., \& Norman, M. L. 1998, ApJ, 495, 80

Bullock, J. S., Dekel, A., Kolatt, T. S., Kravtsov, A. V., Klypin, A. A., Porciani, C., \& Primack, J. R. 2001, ApJ, 555, 240 
Buote, D. A. 2001, ApJ, 553, L15

Byram, E. T., Chubb, T. A., \& Friedman, H. 1966, Science, 152, 66

Carlstrom, J. E., Holder, G. P., \& Reese, E. D. 2002, ARA\&A, 40, 643

Carlstrom, J. E., Joy, M. K., Grego, L., Holder, G. P., Holzapfel, W. L., Mohr, J. J., Patel, S., \& Reese, E. D. 2000, Physica Scripta Volume T, 85, 148

Cassano, R., Brunetti, G., Röttgering, H. J. A., \& Brüggen, M. 2010, A\&A, 509, A68+

Cavagnolo, K. W., Donahue, M., Voit, G. M., \& Sun, M. 2008a, ArXiv e-prints, 803

-. 2008b, ApJ, 682, 821

Cavaliere, A. G., Gursky, H., \& Tucker, W. H. 1971, Nature, 231, 437

Churazov, E., et al. 2007, A\&A, 467, 529

Clarke, T. E., Blanton, E. L., \& Sarazin, C. L. 2004, ApJ, 616, 178

Cohn, J. D., \& Kadota, K. 2005, ApJ, 632, 1

Colafrancesco, S., \& Marchegiani, P. 2008, A\&A, 484, 51

Crain, R. A., Eke, V. R., Frenk, C. S., Jenkins, A., McCarthy, I. G., Navarro, J. F., \& Pearce, F. R. 2007, MNRAS, 377, 41

da Silva, A. C., Kay, S. T., Liddle, A. R., \& Thomas, P. A. 2004, MNRAS, 348, 1401

De Luca, A., \& Molendi, S. 2004, A\&A, 419, 837

Deiss, B. M., Reich, W., Lesch, H., \& Wielebinski, R. 1997, A\&A, 321, 55 
Dickey, J. M., \& Lockman, F. J. 1990, ARA\&A, 28, 215

Domainko, W., Nedbal, D., Hinton, J. A., \& Martineau-Huynh, O. 2009, International Journal of Modern Physics D, 18, 1627

Eckert, D., Neronov, A., Courvoisier, T. J.-L., \& Produit, N. 2007a, A\&A, 470, 835

Eckert, D., Produit, N., Neronov, A., \& Courvoisier, T. J. . 2007b, ArXiv e-prints, 710

Eckert, D., Produit, N., Paltani, S., Neronov, A., \& Courvoisier, T. J.-L. 2008, A\&A, 479,27

Einstein, A. 1917, Sitzungsberichte der Königlich Preußischen Akademie der Wissenschaften (Berlin), Seite 142-152., 142

Eke, V. R., Navarro, J. F., \& Frenk, C. S. 1998, ApJ, 503, 569

Felten, J. E., Gould, R. J., Stein, W. A., \& Woolf, N. J. 1966, ApJ, 146, 955

Feretti, L., Dallacasa, D., Giovannini, G., \& Tagliani, A. 1995, A\&A, 302, 680

Fermi, E. 1949, Physical Review, 75, 1169

Finoguenov, A., Briel, U. G., Henry, J. P., Gavazzi, G., Iglesias-Paramo, J., \& Boselli, A. 2004, A\&A, 419, 47

Finoguenov, A., Sarazin, C. L., Nakazawa, K., Wik, D. R., \& Clarke, T. E. 2010, ApJ, 715, 1143

Forman, W., Kellogg, E., Gursky, H., Tananbaum, H., \& Giacconi, R. 1972, ApJ, 178,309 
Frontera, F., et al. 2007, ApJ, 666, 86

Fujita, Y., et al. 2008, PASJ, 60, 1133

Fusco-Femiano, R., dal Fiume, D., Feretti, L., Giovannini, G., Grandi, P., Matt, G., Molendi, S., \& Santangelo, A. 1999, ApJ, 513, L21

Fusco-Femiano, R., Landi, R., \& Orlandini, M. 2005, ApJ, 624, L69

—. 2007, ApJ, 654, L9

Fusco-Femiano, R., Orlandini, M., Brunetti, G., Feretti, L., Giovannini, G., Grandi, P., \& Setti, G. 2004, ApJ, 602, L73

Gavazzi, G., \& Trinchieri, G. 1983, ApJ, 270, 410

Giacconi, R., Murray, S., Gursky, H., Kellogg, E., Schreier, E., \& Tananbaum, H. 1972, ApJ, 178, 281

Giovannini, G., \& Feretti, L. 2000, New A, 5, 335

Giovannini, G., Feretti, L., Venturi, T., Kim, K.-T., \& Kronberg, P. P. 1993, ApJ, 406,399

Govoni, F., \& Feretti, L. 2004, International Journal of Modern Physics D, 13, 1549

Gruber, D. E., Matteson, J. L., Peterson, L. E., \& Jung, G. V. 1999, ApJ, 520, 124

Gunn, J. E., \& Gott, III, J. R. 1972, ApJ, 176, 1

Haiman, Z., Mohr, J. J., \& Holder, G. P. 2001, ApJ, 553, 545

Hallman, E. J., O’Shea, B. W., Burns, J. O., Norman, M. L., Harkness, R., \& Wagner, R. 2007, ApJ, 671, 27 
Harris, D. E., \& Romanishin, W. 1974, ApJ, 188, 209

Harrison, F. A., et al. 2005, Experimental Astronomy, 20, 131

Henriksen, M., \& Mushotzky, R. 2001, ApJ, 553, 84

Henriksen, M. J., \& Mushotzky, R. F. 1986, ApJ, 302, 287

Henry, J. P., \& Arnaud, K. A. 1991, ApJ, 372, 410

Högbom, J. A. 1974, A\&AS, 15, 417

Holder, G., Haiman, Z., \& Mohr, J. J. 2001, ApJ, 560, L111

Holder, G., McCarthy, I. G., \& Babul, A. 2007, ArXiv Astrophysics e-prints

Honda, H., Hirayama, M., Ezawa, H., Kikuchi, K., Ohashi, T., Watanabe, M., Kunieda, H., \& Yamashita, K. 1998, in IAU Symposium, Vol. 188, The Hot Universe, ed. K. Koyama, S. Kitamoto, \& M. Itoh, 308-+

Hubble, E. P. 1925, The Observatory, 48, 139

Hudson, D. S., Mittal, R., Reiprich, T. H., Nulsen, P. E. J., Andernach, H., \& Sarazin, C. L. 2010, A\&A, 513, A37+

Hughes, J. P., Butcher, J. A., Stewart, G. C., \& Tanaka, Y. 1993, ApJ, 404, 611

Kalberla, P. M. W., Burton, W. B., Hartmann, D., Arnal, E. M., Bajaja, E., Morras, R., \& Pöppel, W. G. L. 2005, A\&A, 440, 775

Kawano, N., et al. 2009, PASJ, 61, 377

Kellogg, E., Gursky, H., Tananbaum, H., Giacconi, R., \& Pounds, K. 1972, ApJ, 174, L65+ 
Kempner, J. C., \& Sarazin, C. L. 2000, ApJ, 530, 282

Keshet, U., Markevitch, M., Birnboim, Y., \& Loeb, A. 2010, ApJ, 719, L74

Kirsch, M. G., et al. 2005, in Society of Photo-Optical Instrumentation Engineers (SPIE) Conference Series, Vol. 5898, Society of Photo-Optical Instrumentation Engineers (SPIE) Conference Series, ed. O. H. W. Siegmund, 22-33

Kitayama, T., \& Suto, Y. 1996, ApJ, 469, 480

Koutroumpa, D., Lallement, R., Kharchenko, V., \& Dalgarno, A. 2009, Space Sci. Rev., 143, 217

Kravtsov, A. V., Vikhlinin, A., \& Nagai, D. 2006, ApJ, 650, 128

Kushnir, D., \& Waxman, E. 2010, J. Cosmology Astropart. Phys., 2, 25

Lacey, C., \& Cole, S. 1993, MNRAS, 262, 627

LaRoque, S. J., Bonamente, M., Carlstrom, J. E., Joy, M. K., Nagai, D., Reese, E. D., \& Dawson, K. S. 2006, ApJ, 652, 917

Lau, E. T., Kravtsov, A. V., \& Nagai, D. 2009, ApJ, 705, 1129

Lemson, G., \& Springel, V. 2006, in Astronomical Society of the Pacific Conference Series, Vol. 351, Astronomical Data Analysis Software and Systems XV, ed. C. Gabriel, C. Arviset, D. Ponz, \& S. Enrique, 212-+

Loken, C., Norman, M. L., Nelson, E., Burns, J., Bryan, G. L., \& Motl, P. 2002, ApJ, 579,571

Longair, M. S. 1994, High energy astrophysics. Vol.2: Stars, the galaxy and the interstellar medium, ed. Longair, M. S. 
Lukić, Z., Heitmann, K., Habib, S., Bashinsky, S., \& Ricker, P. M. 2007, ApJ, 671, 1160

Lumb, D. H., Finoguenov, A., Saxton, R., Aschenbach, B., Gondoin, P., Kirsch, M., \& Stewart, I. M. 2003, in Presented at the Society of Photo-Optical Instrumentation Engineers (SPIE) Conference, Vol. 4851, X-Ray and Gamma-Ray Telescopes and Instruments for Astronomy. Edited by Joachim E. Truemper, Harvey D. Tananbaum. Proceedings of the SPIE, Volume 4851, pp. 255-263 (2003)., ed. J. E. Truemper \& H. D. Tananbaum, 255-263

Lumb, D. H., Warwick, R. S., Page, M., \& De Luca, A. 2002, A\&A, 389, 93

Lutovinov, A. A., Vikhlinin, A., Churazov, E. M., Revnivtsev, M. G., \& Sunyaev, R. A. 2008, ArXiv e-prints, 802

Madsen, K., Harrison, F., Koglin, J., Mao, P., Craig, W., Pivovaroff, M., \& Christensen, F. 2009, in Bulletin of the American Astronomical Society, Vol. 41, Bulletin of the American Astronomical Society, 347-+

Mantz, A., Allen, S. W., Ebeling, H., \& Rapetti, D. 2008, MNRAS, 387, 1179

Markevitch, M., Forman, W. R., Sarazin, C. L., \& Vikhlinin, A. 1998, ApJ, 503, 77

Markevitch, M., \& Vikhlinin, A. 2007, Phys. Rep., 443, 1

Markwardt, C. B. 2009, in Astronomical Society of the Pacific Conference Series, Vol. 411, Astronomical Society of the Pacific Conference Series, ed. D. A. Bohlender, D. Durand, \& P. Dowler, 251-+

Marshall, F. E., Boldt, E. A., Holt, S. S., Miller, R. B., Mushotzky, R. F., Rose, L. A., Rothschild, R. E., \& Serlemitsos, P. J. 1980, ApJ, 235, 4 
McNamara, B. R., \& Nulsen, P. E. J. 2007, ARA\&A, 45, 117

McNamara, B. R., Nulsen, P. E. J., Wise, M. W., Rafferty, D. A., Carilli, C., Sarazin, C. L., \& Blanton, E. L. 2005, Nature, 433, 45

Million, E. T., \& Allen, S. W. 2009, MNRAS, 399, 1307

Mitchell, R. J., Culhane, J. L., Davison, P. J. N., \& Ives, J. C. 1976, MNRAS, 175, 29P

Mitsuda, K., et al. 2007, PASJ, 59, 1

Moretti, A., Campana, S., Lazzati, D., \& Tagliaferri, G. 2003, ApJ, 588, 696

Motl, P. M., Hallman, E. J., Burns, J. O., \& Norman, M. L. 2005, ApJ, 623, L63

Murgia, M., Govoni, F., Feretti, L., Giovannini, G., Dallacasa, D., Fanti, R., Taylor, G. B., \& Dolag, K. 2004, A\&A, 424, 429

Murgia, M., Govoni, F., Markevitch, M., Feretti, L., Giovannini, G., Taylor, G. B., \& Carretti, E. 2009, A\&A, 499, 679

Mushotzky, R. F. 2004, Clusters of Galaxies: Probes of Cosmological Structure and Galaxy Evolution, 123

Nagai, D. 2006, ApJ, 650, 538

Nakazawa, K., et al. 2007, Progress of Theoretical Physics Supplement, 169, 45

-. 2009, PASJ, 61, 339

Navarro, J. F., Frenk, C. S., \& White, S. D. M. 1995, MNRAS, 275, 720

-. 1997, ApJ, 490, 493 
Neumann, D. M., Lumb, D. H., Pratt, G. W., \& Briel, U. G. 2003, A\&A, 400, 811

Nevalainen, J., Oosterbroek, T., Bonamente, M., \& Colafrancesco, S. 2004, ApJ, 608, 166

O'Dea, C. P., \& Owen, F. N. 1985, AJ, 90, 927

Peebles, P. J. E. 1993, Principles of physical cosmology, ed. Peebles, P. J. E.

Percival, W. J. 2005, A\&A, 443, 819

Perlmutter, S., et al. 1999, ApJ, 517, 565

Petrosian, V. 2001, ApJ, 557, 560

Petrosian, V., \& Bykov, A. M. 2008, Space Sci. Rev., 134, 207

Poole, G. B., Babul, A., McCarthy, I. G., Fardal, M. A., Bildfell, C. J., Quinn, T., \& Mahdavi, A. 2007, ArXiv Astrophysics e-prints

Poole, G. B., Fardal, M. A., Babul, A., McCarthy, I. G., Quinn, T., \& Wadsley, J. 2006, MNRAS, 373, 881

Press, W. H., \& Schechter, P. 1974, ApJ, 187, 425

Randall, S. W., Sarazin, C. L., \& Ricker, P. M. 2002, ApJ, 577, 579, (RSR)

Read, A. M., \& Ponman, T. J. 2003, A\&A, 409, 395

Reid, B. A., \& Spergel, D. N. 2006, ApJ, 651, 643

Reiprich, T. H., \& Böhringer, H. 2002, ApJ, 567, 716

Renaud, M., Bélanger, G., Paul, J., Lebrun, F., \& Terrier, R. 2006a, A\&A, 453, L5 
Renaud, M., Gros, A., Lebrun, F., Terrier, R., Goldwurm, A., Reynolds, S., \& Kalemci, E. 2006b, A\&A, 456, 389

Rephaeli, Y. 1977, ApJ, 212, 608

-. 1979, ApJ, 227, 364

-. 1995, ARA\&A, 33, 541

Rephaeli, Y., \& Gruber, D. 2002, ApJ, 579, 587

Rephaeli, Y., Gruber, D., \& Arieli, Y. 2006, ApJ, 649, 673

Rephaeli, Y., Ulmer, M., \& Gruber, D. 1994, ApJ, 429, 554

Revnivtsev, M., Gilfanov, M., Sunyaev, R., Jahoda, K., \& Markwardt, C. 2003, A\&A, 411,329

Ricker, P. M., \& Sarazin, C. L. 2001, ApJ, 561, 621, (RS)

Ritchie, B. W., \& Thomas, P. A. 2002, MNRAS, 329, 675

Rossetti, M., \& Molendi, S. 2004, A\&A, 414, L41

Rybicki, G. B., \& Lightman, A. P. 1979, Radiative processes in astrophysics, ed. Rybicki, G. B. \& Lightman, A. P.

Sarazin, C. L. 1988, X-ray emission from clusters of galaxies, ed. Sarazin, C. L.

-. 1999, ApJ, 520, 529

Sarazin, C. L. 2002, in Astrophysics and Space Science Library, Vol. 272, Merging Processes in Galaxy Clusters, ed. L. Feretti, I. M. Gioia, \& G. Giovannini, 1-38 
Sato, K., Matsushita, K., Ishisaki, Y., Yamasaki, N. Y., Ishida, M., \& Ohashi, T. 2009, PASJ, 61, 353

Schuecker, P., Böhringer, H., Reiprich, T. H., \& Feretti, L. 2001, A\&A, 378, 408

Schuecker, P., Finoguenov, A., Miniati, F., Böhringer, H., \& Briel, U. G. 2004, A\&A, 426,387

Shafer, R. A. 1983, PhD thesis, National Aeronautics and Space Administration. Goddard Space Flight Center, Greenbelt, MD.

Sheth, R. K., \& Tormen, G. 1999, MNRAS, 308, 119

Skillman, S. W., O’Shea, B. W., Hallman, E. J., Burns, J. O., \& Norman, M. L. 2008, ApJ, 689, 1063

Smith, R. K., Brickhouse, N. S., Liedahl, D. A., \& Raymond, J. C. 2001, ApJ, 556, L91

Snowden, S. L., McCammon, D., Burrows, D. N., \& Mendenhall, J. A. 1994, ApJ, 424,714

Snowden, S. L., Mushotzky, R. F., Kuntz, K. D., \& Davis, D. S. 2008, A\&A, 478, 615

Springel, V., et al. 2005, Nature, 435, 629

Staniszewski, Z., et al. 2009, ApJ, 701, 32

Sugawara, C., Takizawa, M., \& Nakazawa, K. 2009, PASJ, 61, 1293

Sunyaev, R. A., \& Zel'dovich, Y. B. 1972, Comments on Astrophysics and Space Physics, 4, 173 
Takahashi, T., et al. 2007, PASJ, 59, 35

Takei, Y., Henry, J. P., Finoguenov, A., Mitsuda, K., Tamura, T., Fujimoto, R., \& Briel, U. G. 2007, ApJ, 655, 831

Taylor, G. B., Barton, E. J., \& Ge, J. 1994, AJ, 107, 1942

Thierbach, M., Klein, U., \& Wielebinski, R. 2003, A\&A, 397, 53

Tueller, J., et al. 2010, ApJS, 186, 378

Vanderlinde, K., et al. 2010, ArXiv e-prints

Vazza, F., Brunetti, G., \& Gheller, C. 2009, MNRAS, 395, 1333

Venturi, T., Giovannini, G., \& Feretti, L. 1990, AJ, 99, 1381

Vikhlinin, A., Kravtsov, A., Forman, W., Jones, C., Markevitch, M., Murray, S. S., \& Van Speybroeck, L. 2006, ApJ, 640, 691

Vikhlinin, A., et al. 2009, ApJ, 692, 1060

Voit, G. M. 2005, Reviews of Modern Physics, 77, 207

Wang, L., \& Steinhardt, P. J. 1998, ApJ, 508, 483

Watanabe, M., Yamashita, K., Furuzawa, A., Kunieda, H., Tawara, Y., \& Honda, H. 1999, ApJ, 527, 80

Weisskopf, M. C., Guainazzi, M., Jahoda, K., Shaposhnikov, N., O’Dell, S. L., Zavlin, V. E., Wilson-Hodge, C., \& Elsner, R. F. 2010, ApJ, 713, 912

Weller, J., Battye, R. A., \& Kneissl, R. 2002, Phys. Rev. Lett., 88, 231301

White, S. D. M., Navarro, J. F., Evrard, A. E., \& Frenk, C. S. 1993, Nature, 366, 429 
Wik, D. R., Sarazin, C. L., Finoguenov, A., Matsushita, K., Nakazawa, K., \& Clarke, T. E. 2009, ApJ, 696, 1700

Wik, D. R., Sarazin, C. L., Ricker, P. M., \& Randall, S. W. 2008, ApJ, 680, 17

Willson, M. A. G. 1970, MNRAS, 151, 1

Winter, L. M., Mushotzky, R. F., Reynolds, C. S., \& Tueller, J. 2009, ApJ, 690, 1322

Wolf, M. 1906, Astronomische Nachrichten, 170, 211

Wong, K., \& Sarazin, C. L. 2009, ApJ, 707, 1141

Zhang, Y., Böhringer, H., Finoguenov, A., Ikebe, Y., Matsushita, K., Schuecker, P., Guzzo, L., \& Collins, C. A. 2006, A\&A, 456, 55

Zhang, Y., Reiprich, T. H., Finoguenov, A., Hudson, D. S., \& Sarazin, C. L. 2009, ApJ, 699, 1178

Zwicky, F. 1933, Helvetica Physica Acta, 6, 110 